Charlton Okama de Souza

\title{
FORMULAÇÃO HÍBRIDA-TREFFTZ COM ENRIQUECIMENTO SELETIVO: APLICAÇÃO A PROBLEMAS BIDIMENSIONAIS DA ELASTICIDADE
}

Dissertação apresentada à Escola de Engenharia de São Carlos, Universidade de São Paulo, como parte dos requisitos para a obtenção do título de Mestre em Engenharia de Estruturas.

Orientador: Prof. Tit. Sergio Persival Baroncini Proença

São Carlos 
AUTORIZO A REPRODUÇÃO E DIVULGAÇÃO TOTAL OU PARCIAL DESTE TRABALHO, POR QUALQUER MEIO CONVENCIONAL OU ELETRÔNICO, PARA FINS DE ESTUDO E PESQUISA, DESDE QUE CITADA A FONTE.

Ficha catalográfica preparada pela Seção de Tratamento da Informação do Serviço de Biblioteca - EESC/USP

Souza, Charlton Okama de

Formulação híbrida-Trefftz com enriquecimento seletivo : aplicação a problemas bidimensionais da elasticidade / Charlton Okama de Souza ; orientador Sergio Persival Baroncini Proença. -- São Carlos, 2008.

Dissertação (Mestrado-Programa de Pós-Graduação e Área de Concentração em Engenharia de Estruturas) -- Escola de Engenharia de São Carlos da Universidade de São Paulo, 2008 .

1. Formulação híbrida-Trefftz de tensão. 2. Método dos elementos finitos. 3. Método dos elementos finitos generalizados. 4. Método da partição. I. Titulo. 


\section{FOLHA DE JULGAMENTO}

Candidato: Engenheiro CHARLTON OKAMA DE SOUZA

Dissertação defendida e julgada em 14/08/2008 perante a Comissão Julgadora:

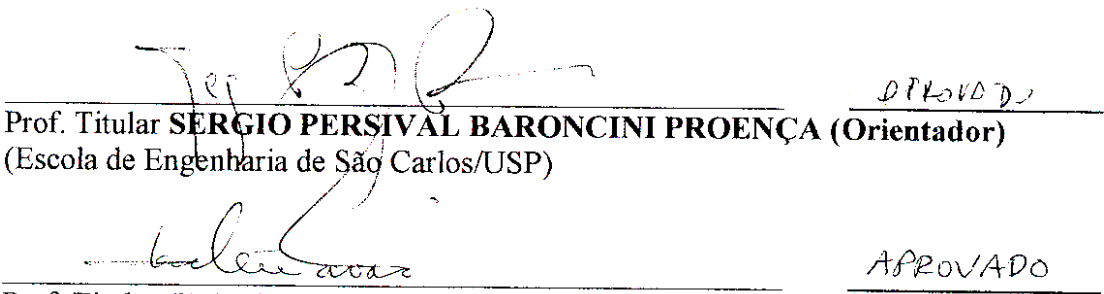

Prof. Titular WALTER SAVASSI

(Escola de Engenharia de São Carlos/USP)

$$
\text { Perumulfo }
$$

Prof. Dr. RENATO PAVANELLO

(Universidade Estadual de Campinas/UNICAMP)

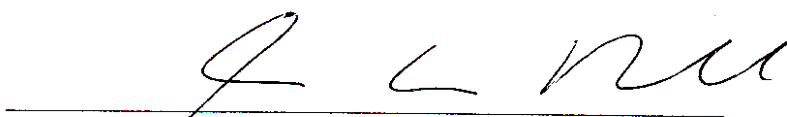

Prof. Assogiado MARCIO ANTONIO RAMALHO

Coordenador do Programa de Pós-Graduação em

Engenharia Civil (Engenharia de Estruturas)

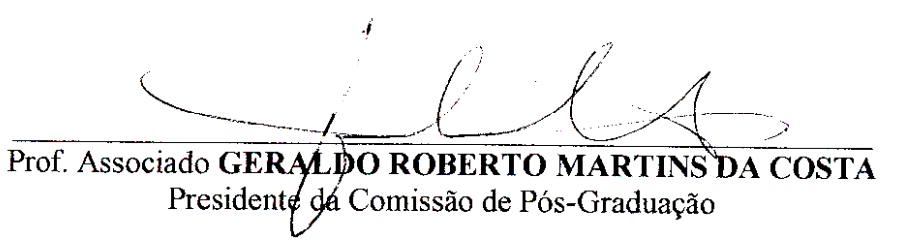


Dedico este trabalho aos meus avós maternos Sintasu e Ushi (in memoriam), que apesar de toda dificuldade inerente a um casal de imigrantes conseguiram, com dignidade, incutir valores morais no âmago de nossa família. 


\section{AGRADECIMENTOS}

Aos meus pais Odete e Sebastião por tudo que me ensinaram e pelo amor incondicional existente.

Aos meus irmãos Charles e Charleston pelo vínculo de afeto e compaixão que nos une e nos fortalece, principalmente, nos momentos adversos.

Ao amigo, professor Antônio Carlos de Matos da Fundação Universidade Federal de Viçosa, pelo papel fundamental que exerceu no período de minha graduação, por ser um dos grandes motivadores em meus estudos de pós-graduação, e, sobretudo, pela maneira que me ensinou encarar a vida.

Ao professor Sergio Persival Baroncini Proença pela amizade, oportunidade concedida e pelo seu profissionalismo; demonstrado tanto como professor, atestado pelo nível de excelência atingido em suas aulas, quanto como orientador, função que desempenha com muito prazer e destreza, sempre disposto a ajudar.

Aos grandes amigos de Viçosa (“chicretada"): Antônio, Russo \& Téia, Beto, Gerson, Dhione, Doro, Lilia e Molha; pelo acolhimento, solicitude e incentivos constantes nas minhas idas e vindas, São Carlos-Viçosa.

Aos amigos do mestrado pelos agradáveis momentos compartilhados neste período de pós-graduação. Em especial à Karla Miller e Rodrigo Couto pelo companheirismo e trocas de experiências no decorrer do primeiro ano de mestrado e Raimundo Amorim, por sua gentileza e auxílio concedidos na fase final de conclusão da dissertação.

À Dora Rohrer por ter me acolhido em São Carlos como um filho, uma amiga inesquecível.

Ao amigo Ricardo pelo relacionamento harmonioso no período em que moramos juntos em São Carlos e a seu pai Ronaldo pelos conselhos fornecidos.

Aos meus vizinhos Magali e Antenor pela ajuda concedida ao longo destes dois anos, sobretudo, nos momentos difíceis que enfrentei em São Carlos.

Aos funcionários do Departamento de Engenharia de Estruturas da Escola de Engenharia de São Carlos (EESC), da biblioteca da EESC e da área de saúde.

À CAPES pelo apoio financeiro, sem o qual esta pesquisa não poderia ter sido concebida.

Aos professores Walter Savassi e Renato Pavanello, membros da banca examinadora, pelas críticas e sugestões apresentadas, as quais, na medida do possível, foram incorporadas ao trabalho.

Principalmente, ao criador, Deus, por minha vida e pelas pessoas que a preencheram com tanta amizade e amor. 
“Muitas coisas não ousamos empreender por parecerem difíceis; entretanto, são difíceis porque não ousamos empreendê-las"

Sêneca 


\section{RESUMO}

SOUZA, C. O. (2008). Formulação híbrida-Trefftz com enriquecimento seletivo: aplicação a problemas bidimensionais da Elasticidade. Dissertação (Mestrado) - Escola de Engenharia de São Carlos, Universidade de São Paulo, São Carlos.

Este trabalho insere-se no âmbito das formulações não convencionais em elementos finitos. Particularmente, introduzem-se alguns aspectos do Método dos Elementos Finitos Generalizados (MEFG) e do clássico refino-p na consagrada formulação híbrida-Trefftz de tensão para a Elasticidade Bidimensional. A formulação apresentada aproxima diretamente dois campos independentes: o de tensões no domínio dos elementos e o de deslocamentos nas fronteiras dos elementos. Baseado na estrutura de enriquecimento centrada em nuvens, proposta pelo MEFG, podem ser selecionadas oportunamente regiões, formadas por um conjunto de elementos e fronteiras de elementos, onde o espaço da aproximação é adequadamente enriquecido mediante o refino-p. Neste contex to campos auto-equilibrados de tensões, derivados da solução da equação de Navier, são utilizados para compor a aproximação no domínio dos elementos, enquanto nas fronteiras dos elementos o campo de deslocamentos é construído a partir de bases específicas de aproximação; seja a base inicial, formada por funções de forma lineares, ou bases enriquecidas com polinômios hierárquicos, não hierárquicos e funções trigonométricas. Aborda-se também, ainda que preliminarmente, um estudo de painéis com múltiplas fissuras pelo Método da Partição em formulação híbridaTrefftz com enriquecimento seletivo. As análises numéricas realizadas revelaram, em geral, uma formulação de ótimo desempenho, caracterizada por uma notável capacidade de aproximação dos campos de tensões e deslocamentos, elevada robustez numérica e reduzido dispêndio computacional.

Palavras-chave: Formulação híbrida-Trefftz de tensão, Método dos Elementos Finitos, Método dos Elementos Finitos Generalizados, Método da Partição. 


\section{ABSTRACT}

SOUZA, C. O. (2008). The Hybrid-Trefftz formulation with selective enrichment: application to two-dimensional problems in elasticity. M.Sc. Dissertation - Escola de Engenharia de São Carlos, Universidade de São Paulo, São Carlos.

This work is inserted in the context of unconventional formulations in the Finite Elements Method. Particularly, some aspects of the Generalized Finite Elements Method (GFEM) and the classic p-refinement are introduced in the well known hybrid-Trefftz stress formulation for the two dimensional elasticity. The presented formulation approximates two independent fields: the one of stresses in the elements domain and the one of displacements in the boundaries of the elements. Based on the enrichment structure centered in clouds, proposed by the GFEM, some regions, formed by a group of elements and boundaries of elements where the approximation space is adequately enriched by the p-refinement, can be opportunely selected. In this context, self-equilibrated stress fields, derived from the solution of the Navier equation, are used to compose the approximation in the elements domain, whereas the displacements field in the borders of the elements is built from specific approximation bases, that is, the initial base formed by linear shape functions, or, bases enriched with hierarchical polynomials, nonhierarchical ones and trigonometric functions. Also, although preliminarily, a study of the multiple-cracked panels is done using the Splitting Method with a hybrid-Trefftz formulation and a selective enrichment. The numeric analyses done revealed, in general, a high performance formulation characterized by a great capacity of approximation the stress fields and displacements, high numeric robustness and reduced computer expenditure.

Keywords: hybrid-Trefftz stress formulation, Finite Elements Method, Generalized Finite Elements Method, Splitting Method 
Figura 2.1. Representação de domínio, fronteiras e forças em um corpo elástico.

Figura 2.2. Sistemas de coordenadas e representação das componentes de forças volúmicas, deslocamentos, deformações e tensões num dado ponto. .11

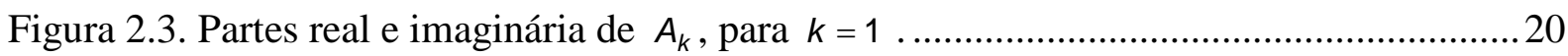

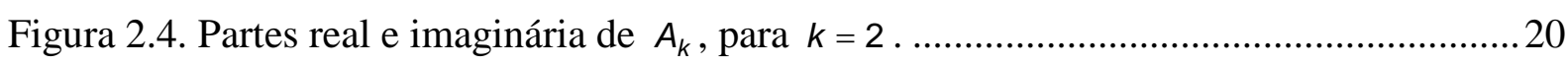

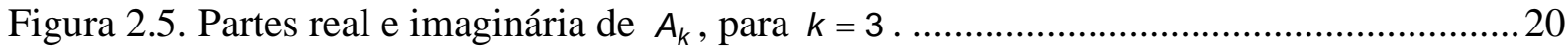

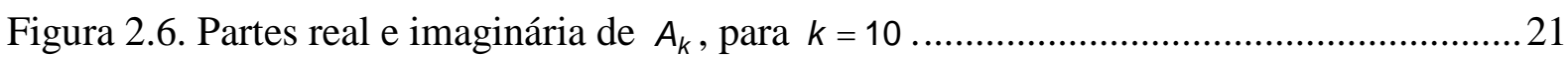

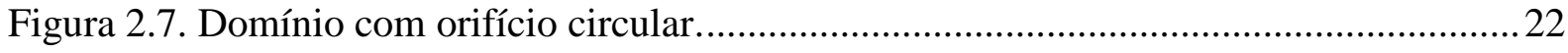

Figura 3.1. (a) HTD: campo aproximado de deslocamentos contínuo em $\Gamma_{\mathrm{e}}$ e (b) HTS: campo aproximado de deslocamentos em $\Gamma_{\mathrm{e}}$ descontínuo nas quinas... 28

Figura 3.2. Elemento finito híbrido-Trefftz quadrilateral de quatro nós e funções de forma

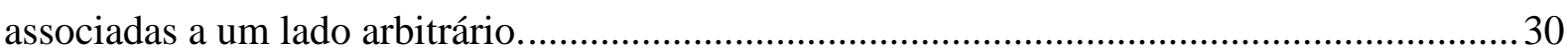

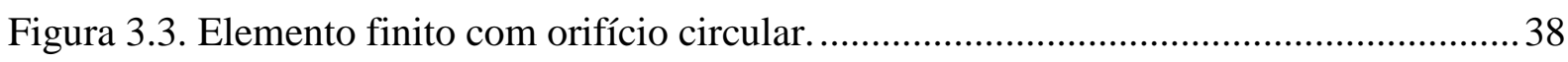

Figura 4.1. Nuvem de influência do enriquecimento seletivo.............................................. 42

Figura 4.2. Base $\beta_{1}$, seqüência com oito termos. ….......................................................... 44

Figura 4.3. Base $\beta_{2}$, sequiência com oito termos............................................................. 44

Figura 4.4. Base $\beta_{3}$, sequiência com oito termos............................................................... 45

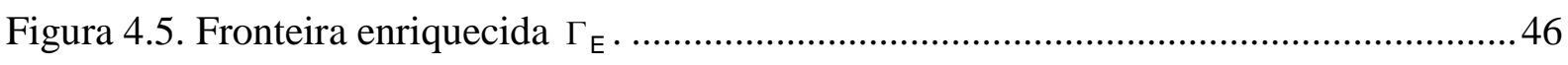

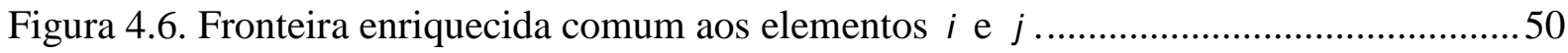

Figura 5.1. Sólido com múltiplas fissuras. ........................................................................53

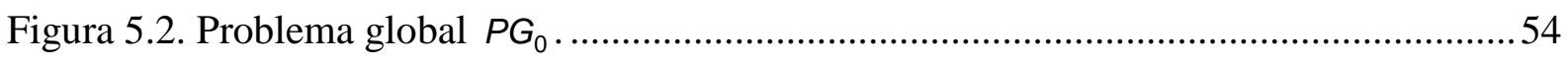

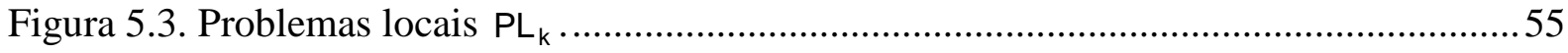

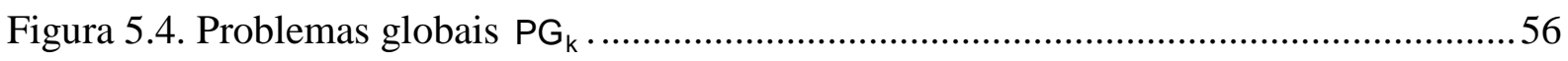

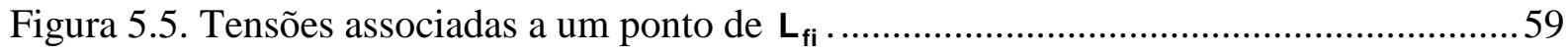

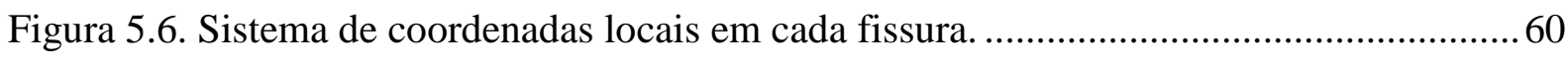

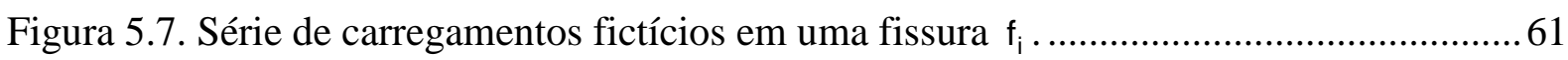

Figura 5.8. Distribuição das forças fictícias numa das faces de uma fissura. ..........................62

Figura 5.9. Pontos nodais considerados na avaliação dos fatores de intensidade de tensão...66 Figura 5.10. Pontos considerados numa análise via MEF para a extração dos fatores de 
intensidade de tensão mediante a técnica de correlação dos deslocamentos.

Figura 6.1. Sistemas de coordenadas no elemento finito com domínio contínuo.................... 70

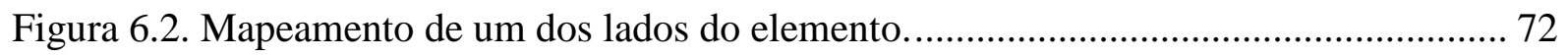

Figura 6.3. Sistemas de coordenadas no elemento finito com orifício. ……......................... 75

Figura 6.4. Mapeamento de um dos lados do elemento com orifício.................................... 76

Figura 7.1. Chapa analisada no $1^{\circ}$ problema. Sua geometria, carregamento e vinculação...... 84

Figura 7.2. Discretização adotada na análise realizada via o MEF clássico para obtenção dos

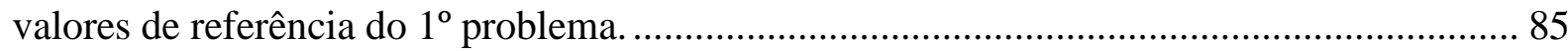

Figura 7.3. Distribuição de tensões de referência do $1^{\circ}$ problema............................................ 85

Figura 7.4. Refino-h. Erro relativo da energia de deformação (\%). ....................................... 86

Figura 7.5. Refino-h. Erro relativo do deslocamento horizontal de referência (\%)................. 87

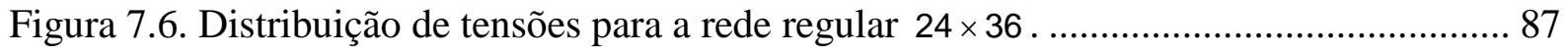

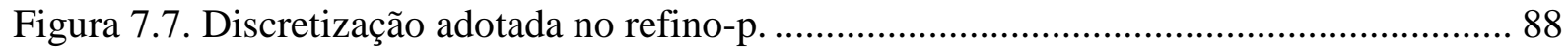

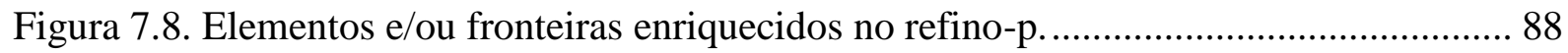

Figura 7.9. Distribuição de tensões obtida quando se enriqueceu apenas o domínio dos elementos

Figura 7.10. Refino-p. Erro relativo da energia de deformação (\%). .................................... 90

Figura 7.11. Refino-p. Erro relativo do deslocamento horizontal de referência (\%)............... 91

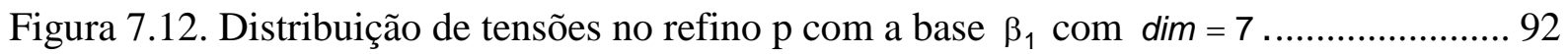

Figura 7.13. Discretizações adotadas no enriquecimento seletivo......................................... 93

Figura 7.14. Região enriquecida da rede $8 \times 9$. a) Cinco elementos enriquecidos e nove fronteiras estáticas e b) Dez elementos enriquecidos e dezoito fronteiras estáticas. ............... 93

Figura 7.15. Distribuição de tensões. a) Situação não enriquecida e b) Enriquecimento com a

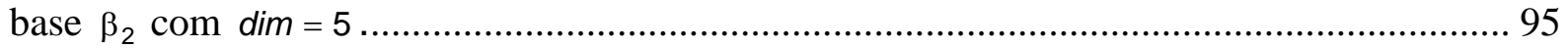

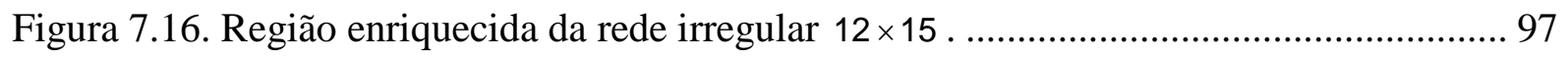

Figura 7.17. Distribuição de tensões. a) Situação não enriquecida e b) Enriquecimento com a

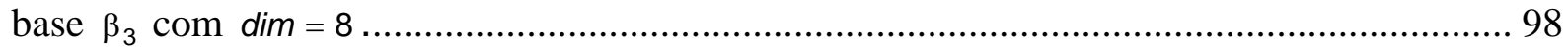

Figura 7.18. Chapa analisada no $2^{\circ}$ problema. Sua geometria, carregamento e vinculação.... 99

Figura 7.19. Discretização adotada na análise realizada via o MEF clássico para obtenção dos

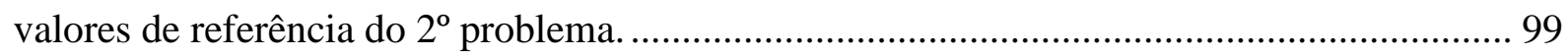

Figura 7.20. Distribuição de tensões de referência do $2^{\circ}$ problema...................................... 100

Figura 7.21. Discretização adotada na análise do $2^{\circ}$ problema............................................. 100

Figura 7.22. Elementos e fronteiras estáticas enriquecidos na discretização adotada. ........... 101 
Figura 7.23. Refino-p. Erro relativo da energia de deformação (\%).................................... 101

Figura 7.24. Distribuição de tensões obtida no refino-p com a base $\beta_{2} \operatorname{com} \operatorname{dim}=7 \ldots \ldots \ldots . .102$

Figura 7.25. Sensibilidade da energia de deformação à distorção da rede............................. 103

Figura 7.26. Sensibilidade da tensão de referência à distorção da rede. …............................. 103

Figura 7.27. Sensibilidade do deslocamento vertical de referência à distorção da rede. ....... 104

Figura 7.28. Chapa analisada no $3^{\circ}$ problema. Sua geometria, carregamento e vinculação. . 104

Figura 7.29. Discretização adotada na análise realizada via o MEF clássico para obtenção dos valores de referência do $3^{\circ}$ problema. 105

Figura 7.30. Distribuição de tensões de referência do $3^{\circ}$ problema. ....................................... 106

Figura 7.31. Discretização adotada no refino-p do $3^{\circ}$ problema. .......................................... 106

Figura 7.32. Elementos e fronteiras estáticas enriquecidos no refino-p................................ 107

Figura 7.33. Refino-p. Erro relativo da energia de deformação (\%).................................... 107

Figura 7.34. Refino-p. Erro relativo do deslocamento horizontal de referência (\%). ............ 108

Figura 7.35. Refino-p. Erro relativo do fator de concentração de tensões (\%). ...................... 108

Figura 7.36. Distribuição de tensões obtida no refino-p com a base $\beta_{1}$ com $\operatorname{dim}=7 \ldots \ldots \ldots . . .109$

Figura 7.37. Fator de concentração de tensões para diferentes relações de $r / e \ldots \ldots \ldots \ldots \ldots . . . . . . .110$

Figura 7.38. Discretização adotado no enriquecimento seletivo.......................................... 110

Figura 7.39. Elementos e fronteiras enriquecidas no enriquecimento seletivo. ..................... 111

Figura 7.40. Distribuição de tensões. a) Situação não enriquecida e b) Enriquecimento com a

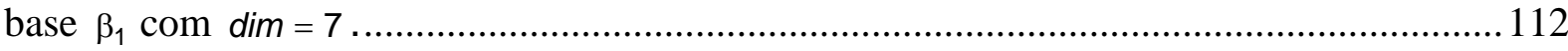

Figura 7.41. Chapa analisada no $4^{\circ}$ problema. Sua geometria e carregamento...................... 113

Figura 7.42. a) Discretização adotada em $P G_{0}$ e nos $P G_{k}$, b) Discretização adotada nos $P L_{k}$ e outros aspectos de interesse e c) Legenda das linhas coloridas........................................... 114

Figura 7.43. Erro relativo do fator de intensidade de tensões (\%). 115 Figura 7.44. a) Distribuição de tensões para o $1^{\circ}$ termo da série de carregamentos fictícios, quando se utilizou a base $\beta_{1}$ sem enriquecimento e b) Distribuição de tensões para o $1^{\circ}$ termo da série de carregamentos fictícios, quando se utilizou a base $\beta_{1}$ com $\operatorname{dim}=7$. 116 Figura 7.45. a) Distribuição de tensões para o $10^{\circ}$ termo da série de carregamentos fictícios, quando se utilizou a base $\beta_{1}$ sem enriquecimento e b) Distribuição de tensões para o $10^{\circ}$ termo da série de carregamentos fictícios, quando se utilizou a base $\beta_{1}$ com $\operatorname{dim}=7$. 116 Figura 7.46. Chapa analisada no $5^{\circ}$ problema. Sua geometria, vinculação e carregamento. . 117 Figura 7.47. Discretização adotada na análise realizada via o MEF clássico para obtenção dos valores de referência do $5^{\circ}$ problema. 
Figura 7.48. Discretizações adotadas na análise comparativa.

Figura 7.49. Refino-h. Erro relativo do deslocamento vertical no ponto de referência (\%).. 121

Figura 7.50. Refino-h. Erro relativo da tensão normal máxima no ponto de referência (\%). 122

Figura C.1. Chapa discretizada em dois elementos. ...................................................... 140

Figura D.1. Chapa discretizada em dois elementos com dois trechos enriquecidos............. 145 
Tabela 7.1 - Resultados do refino-p, quando se enriqueceu apenas o domínio dos elementos 89 Tabela 7.2 - Número total de graus de liberdade envolvidos no refino-p

Tabela 7.3 - Grandezas de interesse aproximadas para algumas situações de enriquecimento, quando se enriqueceu 5 elementos e 9 fronteiras estáticas da rede $8 \times 9$

Tabela 7.4 - Graus de liberdade envolvidos para algumas situações de enriquecimento, quando se enriqueceu 5 elementos e 9 fronteiras estáticas da rede $8 \times 9$

Tabela 7.5 - Grandezas de interesse aproximadas para algumas situações de enriquecimento, quando se enriqueceu 10 elementos e 18 fronteiras estáticas da rede $8 \times 9$

Tabela 7.6 - Graus de liberdade envolvidos para algumas situações de enriquecimento, quando se enriqueceu 10 elementos e 18 fronteiras estáticas da rede $8 \times 9$ .96

Tabela 7.7 - Grandezas de interesse aproximadas para algumas situações de enriquecimento, quando se enriqueceu 14 elementos e 26 fronteiras estáticas da rede $12 \times 15$ 97

Tabela 7.8-Graus de liberdade envolvidos para algumas situações de enriquecimento, quando se enriqueceu 14 elementos e 26 fronteiras estáticas da rede $12 \times 15$

Tabela 7.9 - Grandezas de interesse aproximadas para algumas situações de enriquecimento, quando se enriqueceu 32 elementos e 64 fronteiras estáticas.

Tabela 7.10 - Graus de liberdade envolvidos para algumas situações de enriquecimento, quando se enriqueceu 32 elementos e 64 fronteiras estáticas.

Tabela 7.11 - Resultados aproximados de algumas grandezas de interesse para diferentes formulações em elementos finitos encontradas na literatura

Tabela 7.12 - Erros relativos nas aproximações do deslocamento vertical e tensão máxima nos pontos de referência para diferentes formulações em elementos finitos encontradas na

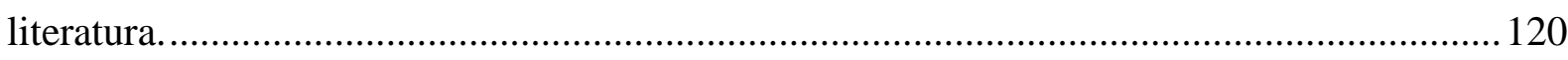

Tabela C.1 - Montagem da matriz dos coeficientes ............................................................. 142

Tabela D.1 - Montagem do sistema resolutivo com enriquecimento 147 
dim - Dimensão das Bases de Aproximação do Campo de Deslocamentos

EPD - Estado Plano de Deformação

EPT - Estado Plano de Tensão

Eq. - Equação

Eqs. - Equações

HTD - Hybrid Trefftz Displacement

HTE - Elemento Hibrido-Trefftz com Enriquecimento

HTS - Hybrid Trefftz Stress

MEC - Método dos Elementos de Contorno

MEF - Método dos Elementos Finitos

MEFG - Método dos Elementos Finitos Generalizados

PVC - Problema de Valor de Contorno

PTV - Princípio dos Trabalhos Virtuais

PTVC - Princípio dos Trabalhos Virtuais Complementares 


\section{LISTA DE SÍMBOLOS}

$\Omega$ - Domínio de um sólido ou estrutura

$\Gamma$ - Contorno de um sólido ou estrutura

$\boldsymbol{b}$ - Vetor de forças volúmicas

$\overline{\boldsymbol{t}}$ - Vetor de forças de superfície prescritas

$\overline{\boldsymbol{u}}$ - Vetor de deslocamentos prescritos

$\Gamma_{t}$ - Fronteira estática de um contorno de um sólido ou estrutura

$\Gamma_{u}$ - Fronteira cinemática de um contorno de um sólido ou estrutura

$L_{c}$ - Matriz formada por operadores diferenciais segundo o sistema cartesiano

$\sigma_{c}-$ Vetor representativo do tensor das tensões segundo o sistema cartesiano

$\sigma_{x}, \sigma_{y}$ e $\tau_{x y}$ - componentes do tensor de tensões segundo o sistema cartesiano

$\boldsymbol{b}_{\boldsymbol{c}}$ - Vetor de forças volúmicas segundo o sistema cartesiano

$b_{x}$ e $b_{y}$ - Componentes das forças volúmicas segundo o sistema cartesiano

$\boldsymbol{M}_{\boldsymbol{\sigma}}$ - Matriz que depende da posição do raio polar, definida na equação de equilíbrio.

$L_{p}$ - Matriz formada por operadores diferenciais segundo o sistema polar

$\sigma_{p}-$ Vetor representativo do tensor das tensões segundo o sistema polar

$\sigma_{\mathrm{r}}, \sigma_{\theta}$ e $\tau_{\mathrm{r} \theta}$ - Componentes do tensor de tensões segundo o sistema polar.

$\boldsymbol{b}_{\boldsymbol{p}}$ - Vetor de forças volúmicas segundo o sistema polar

$b_{r}$ e $b_{\theta}$ - Componentes das forças volúmicas segundo o sistema polar

$\varepsilon_{c}$ - Vetor representativo do tensor das deformações segundo o sistema cartesiano

$\varepsilon_{x}, \varepsilon_{y}$ e $\gamma_{x y}$ - Componentes da deformação linear específica segundo o sistema cartesiano.

$\boldsymbol{u}_{\boldsymbol{c}}$ - Vetor do campo de deslocamentos segundo o sistema cartesiano

$\mathrm{u}_{\mathrm{x}}$ e $\mathrm{u}_{\mathrm{y}}$ - Componentes do campo de deslocamentos segundo o sistema cartesiano

$\varepsilon_{\mathrm{p}}$ - Vetor representativo do tensor das deformações segundo o sistema polar

$\varepsilon_{\mathrm{r}}, \varepsilon_{\theta}$ e $\gamma_{\mathrm{r} \theta}$ - Componentes do tensor das deformações segundo o sistema polar

$\boldsymbol{u}_{\boldsymbol{p}}$ - Vetor do campo de deslocamentos segundo o sistema polar

$\mathrm{u}_{r}$ e $\mathrm{u}_{\theta}$ - Componentes do campo de deslocamentos segundo o sistema cartesiano

$\boldsymbol{M}_{\boldsymbol{\varepsilon}}$ - Matriz que depende da posição do raio polar, definida na equação de compatibilidade

$\boldsymbol{D}$ - Tensor constitutivo de rigidez 
$\boldsymbol{f}$ - Tensor constitutivo de flexibilidade

E - Módulo de Young

$v$ - Coeficiente de Poisson

$\boldsymbol{R}_{u \boldsymbol{\theta}}$ - Matriz de rotação dos deslocamentos

$\boldsymbol{R}_{\sigma \theta}$ - Matriz de rotação das tensões

$\overline{\boldsymbol{u}}_{c}$ - Vetor de deslocamentos prescritos em $\Gamma_{\mathrm{u}}$

$\bar{u}_{x}$ e $\bar{u}_{y}$ - Componentes dos deslocamentos prescritos

$\boldsymbol{A}$ - Matriz formada por co-senos diretores

$n_{x}$ e $n_{y}$ - Componentes de um vetor unitário normal à superfície $\Gamma_{\mathrm{t}}$

$\boldsymbol{t}_{c}$ - Vetor das tensões numa superfície segundo o sistema cartesiano

$t_{x}$ e $t_{y}$-Componentes das tensões em $\Gamma_{t}$

$\overline{\mathbf{t}}_{\mathrm{c}}-$ Vetor de forças de superfície prescritas em $\Gamma_{\mathrm{t}}$

$\bar{t}_{x}$ e $\bar{t}_{y}$ - Componentes das forças de superfície prescritas

$\boldsymbol{u}$ - Campo de deslocamentos num sistema arbitrário de referência

$\boldsymbol{b}$ - Vetor de forças volúmicas num sistema arbitrário de referência

$\mathfrak{R}$ - Operador matricial da equação de Navier

$\lambda_{c}$ e $\lambda_{p}$ - Constantes auxiliares da equação de Navier

û - Vetor representativo da solução particular

$\boldsymbol{u}_{\boldsymbol{h}}$ - Vetor representativo da solução homogênea

$\boldsymbol{u}_{M}$ - Vetor representativo da solução de corpo rígido

c - Vetor formado pelos parâmetros da combinação linear da aproximação da solução homogênea

$\boldsymbol{N}$ - Matriz que coleta os vetores da solução homogênea

$\boldsymbol{N}_{\boldsymbol{j}}$ - Vetores da solução homogênea

$\overline{\boldsymbol{N}}$ - Matriz que coleta os vetores da solução de corpo rígido

$\bar{c}$ - Vetor formado pelos parâmetros da combinação linear da aproximação da solução de corpo rígido

$\boldsymbol{u}_{\boldsymbol{h c}}$ - Vetor representativo da solução homogênea segundo o sistema cartesiano

$\boldsymbol{u}_{M c}$ - Vetor representativo da solução de corpo rígido segundo o sistema cartesiano

$\boldsymbol{N}_{j c}$ - Vetores solução da solução homogênea segundo o sistema cartesiano

$\overline{\boldsymbol{N}}_{j c}$ - Vetores solução da solução homogênea segundo o sistema cartesiano

$\boldsymbol{N}_{c}$ - Matriz que coleta os vetores solução da solução homogênea segundo o sistema cartesiano 
$\overline{\boldsymbol{N}}_{c}$ - Matriz que coleta os vetores solução da solução de corpo rígido segundo o sistema cartesiano

$F(z)$ - Função arbitrária da equação de Muskhelishvili

$G(z)$ - Função arbitrária da equação de Muskhelishvili

z - Número complexo

$\bar{z}$ - Número complexo conjugado

$i$ - Unidade imaginária de um número complexo

$\mathbf{N}_{\mathbf{A K}}$ - Seqüência 1 de vetores da solução homogênea

$\mathbf{N}_{\mathbf{B K}}$ - Seqüência 2 de vetores da solução homogênea

$\mathbf{N}_{\mathbf{C K}_{K}}$ - Seqüência 3 de vetores da solução homogênea

$\mathbf{N}_{\mathbf{D K}_{\mathrm{K}}}$ - Seqüência 4 de vetores da solução homogênea

$A_{k}$ - Funções que definem a seqüência $\mathbf{N}_{\mathbf{A K}}$

$B_{k}$ - Funções que definem a seqüência $\mathbf{N}_{\mathbf{B K}}$

$C_{k}$ - Funções que definem a seqüência $\mathbf{N}_{C_{K}}$

$D_{k}$ - Funções que definem a sequiência $\mathbf{N}_{\mathbf{D K}_{K}}$

$u_{h p}$ - Vetor representativo da solução homogênea segundo o sistema polar

$\boldsymbol{u}_{M p}$ - Vetor representativo da solução de corpo rígido segundo o sistema polar

$\boldsymbol{N}_{j p}$ - Vetores da solução homogênea segundo o sistema polar

$\overline{\boldsymbol{N}}_{j p}$ - Vetores da solução homogênea segundo o sistema polar

$\boldsymbol{N}_{\boldsymbol{p}}$ - Matriz que coleta os vetores da solução homogênea segundo o sistema polar

$\overline{\boldsymbol{N}}_{p}$ - Matriz que coleta os vetores da solução de corpo rígido segundo o sistema polar

$f_{k}(r)$ e $g_{k}(r)$ - funções solução da equação de Euler-Cauchy.

$\boldsymbol{S}_{c}$ - Matriz que coleta as funções de aproximação de tensões derivadas da equação de Navier segundo o sistema cartesiano.

$\boldsymbol{S}_{\boldsymbol{p}}$ - Matriz que coleta as funções de aproximação de tensões derivadas da equação de Navier segundo o sistema polar.

$\boldsymbol{T}_{\boldsymbol{c}}$ - Matriz que coleta as funções de aproximação de tensões numa superfície, derivadas da equação de Navier segundo o sistema cartesiano.

$\boldsymbol{T}_{p}$ - Matriz que coleta as funções de aproximação de tensões numa superfície, derivadas da equação de Navier segundo o sistema polar.

$\tilde{u}$ - Aproximação do campo de deslocamentos

$\xi$ - coordenada adimensional local 
$\tilde{\boldsymbol{U}}$ - Matriz que coleta as funções de forma para a aproximação do campo de deslocamentos

$\boldsymbol{d}$ - Vetor composto pelos graus de liberdade em deslocamentos

$\varphi_{1}$ e $\varphi_{2}$ - Funções de forma lineares

$\Omega_{\mathrm{e}}$ - Domínio do elemento

$\boldsymbol{\Gamma}_{\text {te }}$ - Fronteira estática no contorno do elemento

$\Gamma_{\mathrm{ue}}$ - Fronteira cinemática no contorno do elemento

$\boldsymbol{F}$ - Matriz do sistema resolvente do elemento

G - Matriz do sistema resolvente do elemento

$\boldsymbol{e}$ - Vetor do sistema resolvente do elemento

$\boldsymbol{q}$ - Vetor do sistema resolvente do elemento

$\Gamma_{e}^{i}$ e $\Gamma_{e}^{j}$ - Fronteiras relativas, respectivamente, aos elementos i e j

$\tilde{\boldsymbol{u}}^{i}$ e $\tilde{\boldsymbol{u}}^{j}$ - Distribuição de deslocamentos em $\Gamma_{\mathrm{e}}{ }^{\mathrm{i}}$ e $\Gamma_{\mathrm{e}}{ }^{\mathrm{j}}$, respectivamente

$\boldsymbol{q}_{\boldsymbol{\sigma k l} \mathbf{l}}$ - Forças nodais equivalentes provenientes das tensões

$\boldsymbol{q}_{\bar{t} \boldsymbol{k} \boldsymbol{l}}$ - Forças nodais equivalentes provenientes do carregamento

$\boldsymbol{d}_{\boldsymbol{t}}$ - Vetor que coleta os graus de liberdade nodais em deslocamentos do sistema resolvente global

$\boldsymbol{q}_{\boldsymbol{t}}$ - Vetor de forças nodais equivalentes

$\beta_{0}$ - Base de aproximação inicial

$\beta_{g}$ - Base enriquecida genérica

$\beta_{E}$ - Base de interesse utilizada no enriquecimento

$h_{j}$ - Funções utilizadas no enriquecimento

$\beta_{1}$ - Base enriquecida com refinamento polinomial hierárquico

$\beta_{2}$ - Base enriquecida com refinamento polinomial não hierárquico

$\beta_{3}$ - Base enriquecida envolvendo funções trigonométricas

$\Gamma_{\mathrm{E}}$ - Uma fronteira enriquecida

$\tilde{\boldsymbol{U}}_{E}$ - Matriz que coleta as funções de aproximação numa fronteira enriquecida

$\boldsymbol{d}_{\boldsymbol{E}}$ - Vetor que coleta os graus de liberdade em deslocamentos numa fronteira enriquecida

$\tilde{\boldsymbol{U}}_{\Gamma_{E}}$ - Matriz que coleta as funções de aproximação decorrentes exclusivamente do enriquecimento

$\boldsymbol{d}_{\Gamma_{E}}$ - Vetor que coleta os graus de liberdade em deslocamentos decorrentes exclusivamente do enriquecimento

$\Delta u_{x i}$ e $\Delta u_{y i}$ - Graus de liberdade decorrentes exclusivamente do enriquecimento segundo as 
direções $x$ e $y$

$\boldsymbol{G}_{\boldsymbol{E}}$ - Matriz que coleta as matrizes $\boldsymbol{G}_{\Gamma_{E}}$ e $\Delta \boldsymbol{G}_{\Gamma_{E}}$ do sistema resolutivo com enriquecimento $G_{\Gamma_{E}}-$ Parcela de $G_{E}$ referente à aproximação inicial

$\Delta \boldsymbol{G}_{\Gamma_{E}}$ - Parcela de $\boldsymbol{G}_{\boldsymbol{E}}$ introduzida em virtude do enriquecimento

$\boldsymbol{q}_{\boldsymbol{E}}$ - Vetor que coleta os vetores $\boldsymbol{q}_{\Gamma_{E}}$ e $\Delta \boldsymbol{q}_{\Gamma_{E}}$ do sistema resolutivo com enriquecimento

$\boldsymbol{q}_{\Gamma_{E}}$ - Parcela de $\boldsymbol{q}_{E}$ referente à aproximação inicial

$\Delta \boldsymbol{q}_{\Gamma_{E}}$ - Parcela de $\boldsymbol{q}_{\boldsymbol{E}}$ introduzida em virtude do enriquecimento

$\Delta \boldsymbol{d}$ - Vetor genérico que coleta os graus de liberdade em deslocamentos decorrentes exclusivamente do enriquecimento no elemento

$\Delta \boldsymbol{G}$ - Matriz genérica que coleta as funções de aproximação decorrentes exclusivamente do enriquecimento no elemento

$\Delta \boldsymbol{q}$ - Vetor de forças nodais equivalentes, decorrente exclusivamente do enriquecimento no elemento

$\Delta \boldsymbol{d}_{\boldsymbol{t}}$ - Vetor que coleta os graus de liberdade nodais em deslocamentos decorrentes do enriquecimento do sistema resolvente global

$\Delta \boldsymbol{q}_{\boldsymbol{t}}$ - Vetor que coleta as forças equivalentes das fronteiras enriquecidas.

$f_{i}$ - Fissura arbitrária no domínio

$P G_{0}$ - Designação do problema global básico

$P L_{k}$ - Designação dos problemas locais

$P G_{k}$ - Designação dos problemas globais

$u_{0}$ - Solução do campo de deslocamentos de $P G_{0}$

$\boldsymbol{u}_{\boldsymbol{L} \boldsymbol{k}}$ - Solução do campo de deslocamentos referentes a cada $P \boldsymbol{L}_{\boldsymbol{k}}$

$\boldsymbol{u}_{G \boldsymbol{k}}$ - Solução do campo de deslocamentos referentes a cada $\boldsymbol{P} \boldsymbol{G}_{\boldsymbol{k}}$

$\alpha_{k}$ - Fatores de escala

$\sigma_{0}$ - Solução do campo de tensões de $P G_{0}$

$\boldsymbol{\sigma}_{G k}$ - Solução do campo de tensões referentes a cada $P G_{\boldsymbol{k}}$

$\boldsymbol{\sigma}_{L k}$ - Solução do campo de tensões referentes a cada $P \boldsymbol{L}_{\boldsymbol{k}}$

$\mathrm{L}_{\mathrm{fi}}$ - linha de uma fissura previamente desconsiderada

$\boldsymbol{t}_{\boldsymbol{o}}$ - Distribuição de tensões numa $\mathrm{L}_{\mathrm{fi}}$ do $P G_{0}$

$t_{0 \eta}$ e $t_{0 \zeta}$ - Componentes da tensão $\boldsymbol{t}_{\boldsymbol{0}}$

$V_{f i}$ - Domínio local de influência de uma fissura $f_{i}$

$\Gamma_{\mathrm{fi}}$ - Caminho interno arbitrário de influência de uma fissura $f_{i}$ 
$\boldsymbol{Q}_{L_{j}}$ - Carregamento fictício

$\boldsymbol{t}_{\boldsymbol{j}}$ - Distribuições de tensões ao longo de $\Gamma_{\mathrm{fi}}$

$\boldsymbol{u}_{\boldsymbol{j}}$ - Distribuições de deslocamentos ao longo de $\Gamma_{\mathrm{fi}}$

$\boldsymbol{\alpha}$ - Vetor formado pelos fatores de escala $\alpha_{j}$

$\boldsymbol{t}_{L}$ - Distribuição de tensões nas faces de uma fissura de um $P \boldsymbol{L}_{\boldsymbol{k}}$

$\boldsymbol{Q}_{L}$ - Matriz que coleta os vetores de carregamentos fictícios $\boldsymbol{Q}_{L_{j}}$

$Q_{L j \eta}$ e $Q_{L j \zeta}$ - Componentes de um vetor $\boldsymbol{Q}_{L j}$

$\boldsymbol{t}_{G}$ - Distribuição de tensões numa $\mathbf{L}_{\mathbf{f i}}$ de um $\boldsymbol{P} \boldsymbol{G}_{k}$

$\boldsymbol{Q}_{G_{j}}$ - Vetores de distribuição de tensões numa $\mathbf{L}_{\mathrm{fi}}$ de um $\boldsymbol{P} \boldsymbol{G}_{k}$

$Q_{G_{j \eta}}$ e $Q_{G_{j \zeta}}$ - componentes de um $\boldsymbol{Q}_{G_{j}}$

$\boldsymbol{Q}_{G}$ - Matriz que coleta os vetores $\boldsymbol{Q}_{G_{j}}$

$\boldsymbol{K}_{\boldsymbol{p} i}$ - Matriz dos coeficientes do sistema resolutivo do Método da Partição

$\boldsymbol{v}_{\boldsymbol{p} i}$ - Vetor dos termos independentes do sistema resolutivo do Método da Partição

$\tilde{u}_{0}$ - Solução do campo de deslocamentos de $P G_{0}$ em formulação híbrida-Trefftz

$\tilde{u}_{G \boldsymbol{k}}$ - Solução do campo de deslocamentos referentes a cada $\mathbf{P L _ { \boldsymbol { k } }}$ em formulação híbridaTrefftz

$\tilde{u}_{L k}$ - Solução do campo de deslocamentos referentes a cada $P G_{k}$ em formulação híbridaTrefftz

$\boldsymbol{R}_{\alpha}$ - Matriz de rotação de tensões numa linha

$g_{i}(\eta)$ - Função que indica a distribuição das forças fictícias nas faces de uma fissura

a - Comprimento efetivo de uma fissura (capítulo 5)

$\eta_{1}$ e $\eta_{2}$ - Valores adimensionais especificados para o sistema coordenado da fissura, referentes aos nós 1 e 2 de face de contato com um elemento

$\sigma_{\Gamma j}$ - Vetor representativo da distribuição de tensões dos elementos adjacentes a uma fronteira $\Gamma_{\mathrm{fi}}$

$u_{\eta}$ e $u_{\zeta}$ - Componentes do campo de deslocamentos nas direções paralela e perpendicular às faces da fissura, respectivamente.

$K_{\mathrm{I}}$ e $K_{\mathrm{II}}$ - Fatores de intensidade de tensão, respectivamente, para os modos de abertura e deslizamento.

G- Modulo de elasticidade transversal.

$r$ - Distância de um ponto considerado à extremidade da fissura (capítulo 5)

$\phi$ - Ângulo do ponto considerado relativo à bissetriz da abertura da fissura.

$\kappa$ - Constante auxiliar utilizada nas equações que definem a distribuição do campo de 
deslocamentos no entorno da região de singularidade

$\Delta_{\zeta}$ e $\Delta \eta$ - Deslocamentos relativos dos referidos pontos segundo as direções perpendicular e paralela às faces da fissura, respectivamente

c - Posição do centróide do elemento (capítulo 6)

$X_{i}$ e $Y_{i}$ - Posições de um nó $i$ de um elemento segundo o sistema global de referência

$\bar{X}$ e $\bar{Y}$ - Coordenadas do centróide c do elemento

$A$ - Área do elemento (capítulo 6)

$x_{k}$ e $y_{k}$-Coordenadas cartesianas de um nó k de um elemento segundo o sistema local de coordenadas

$x$ e $y$-Distribuição de posições ao longo de uma fronteira (capítulo 6)

$\boldsymbol{n}$ - Vetor que coleta os co-senos diretores $n_{x}, n_{y}$ e $n_{z}$ de um determinado lado segundo o sistema cartesiano

$d_{N}$ - Distância de normalização

$x_{N}$ - Coordenada adimensional segundo a direção $\mathrm{x}$

$y_{N}$ - Coordenada adimensional segundo a direção y

$\bar{L}_{c}$ - Matriz formada por operadores diferenciais segundo o sistema cartesiano normalizado

$f\left(x_{i}\right)$ e $w_{i}$ - Valores funcionais e pesos de Gauss, respectivamente

$\mathrm{d} \Gamma$ - Diferencial de uma fronteira de um elemento

$r$ - Raio polar (capítulo 6)

$s_{h}$ e $s_{v}$ - Metade do comprimento dos lados horizontal e vertical de um elemento retangular

$\theta$ - Coordenada angular (capítulo 6)

$\gamma$ - ângulo auxiliar de varredura

$r_{N}$ - Coordenada adimensional polar segundo a direção $r$

$\boldsymbol{A}_{G}$ - Matriz dos coeficientes do sistema resolutivo global

$\boldsymbol{F}_{\boldsymbol{G}}, \boldsymbol{G}_{\boldsymbol{G}}$ e $\Delta \boldsymbol{G}_{\boldsymbol{G}}$ - Matrizes resultantes da alocação das matrizes $\boldsymbol{F}, \boldsymbol{G}$ e $\Delta \boldsymbol{G}$ de cada elemento da discretização adotada

$\boldsymbol{B}_{\boldsymbol{G}}$ - Vetor de termos independentes do sistema resolutivo global

$\boldsymbol{e}_{G}, \boldsymbol{q}_{t G}$ e $\Delta \boldsymbol{q}_{t G}$ - Vetores resultantes da alocação dos vetores $\boldsymbol{e}, \boldsymbol{q}$ e $\Delta \boldsymbol{q}$ de cada elemento da discretização adotada.

$\boldsymbol{X}_{G}-$ Vetor das incógnitas do sistema resolutivo global

$\boldsymbol{R}_{G}$ - Vetor resíduo da solução do sistema resolutivo

$\left\|R_{G}\right\|$ - Norma de $\boldsymbol{R}_{G}$

$U$ - Energia de deformação

$\overline{\boldsymbol{F}}_{\boldsymbol{G}}$ - Matriz que coleta as submatrizes $\boldsymbol{F}_{\boldsymbol{i}}$, para $i=1, \ldots, n$, sendo $n$ o número de elementos da 
discretização adotada.

$U_{R E F}$ - Energia de deformação de referência nas análises numéricas (capitulo 7)

$u_{x \text { ref }}$ e $u_{y_{\text {ref }}}$ - Deslocamentos de referência nas análises numéricas (capitulo 7)

$\left(\sigma_{M A X} / W\right)_{\text {REF }}$ - Fator de concentração de tensões de referência (capitulo 7, problema 3)

$\tilde{K}_{\mathrm{I}}$ ref - Fator de intensidade de tensões de referência (capitulo 7, problema 4)

$\sigma_{R E F}$ - Tensão máxima de referência (capitulo 7, problema 5) 
1 Introdução ............................................................................................................................................

1.1 Considerações gerais ............................................................................................... 1

1.2 Objetivos

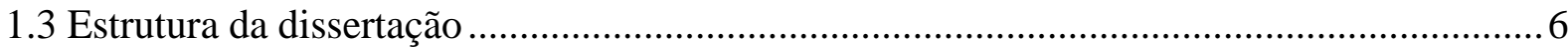

2 Campos aproximados em problemas bidimensionais da Elasticidade: concepção baseada no Método de Trefftz ...............................................................................................8

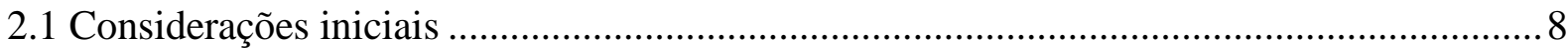

2.2 Relações básicas em problemas de Elasticidade ………………..........................................

2.3 Aproximação do campo de deslocamentos: construção a partir da equação de Navier ..... 15

2.3.1 Aproximação para domínios contínuos ........................................................................... 17

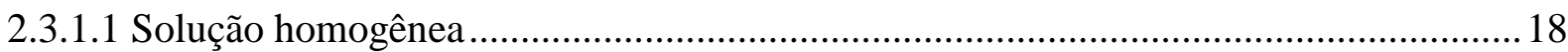

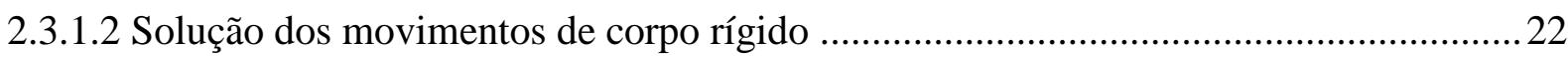

2.3.2 Aproximação para domínios com orifício circular ........................................................22

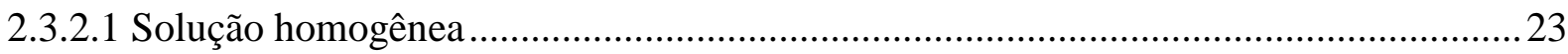

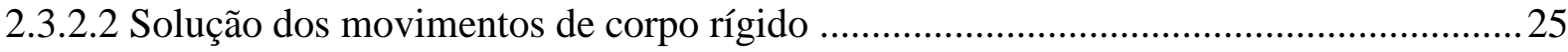

2.4 Aproximação do campo de tensões no domínio ...................................................................26

2.5 Aproximação do campo de tensões no contorno ……………………………………......26

3 Formulação híbrida-Trefftz em problemas bidimensionais da Elasticidade...................28

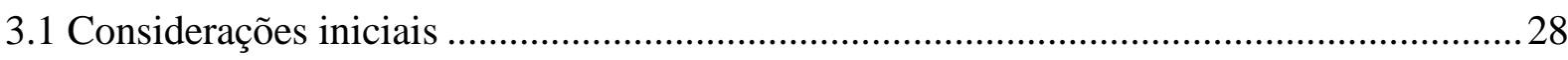

3.2 Elemento finito híbrido-Trefftz em domínios contínuos .....................................................30

3.2.1 Aproximação do campo de tensões .................................................................................. 30

3.2.1.1 Aproximação no domínio do elemento ....................................................................... 30

3.2.1.2 Aproximação no contorno do elemento.................................................................... 31

3.2.2 Aproximação do campo de deslocamentos no contorno ……………………………........ 31

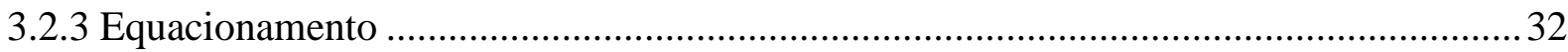

3.2.3.1 Compatibilidade entre deformações no domínio e deslocamentos no contorno ...........33

3.2.3.2 Equilíbrio entre forças internas e externas ....................................................................... 34 
3.2.3.3 Sistema resolutivo

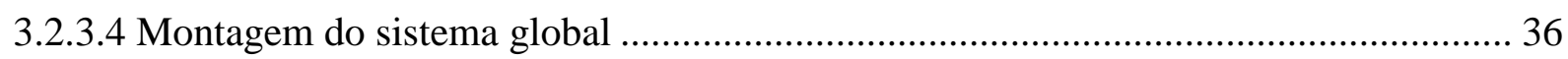

3.3 Elemento finito híbrido-Trefftz em domínios com orifício circular central ...................... 38

4 Formulação híbrida-Trefftz com enriquecimento seletivo em problemas

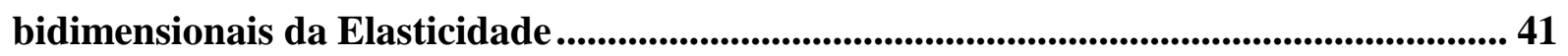

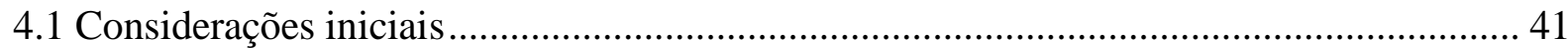

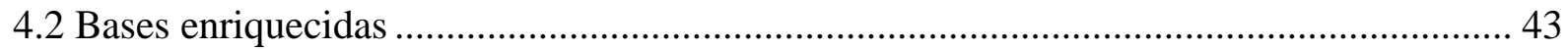

4.3 Aproximação do campo de deslocamentos numa fronteira enriquecida........................... 45

4.4 Equacionamento da formulação com enriquecimento ..................................................... 47

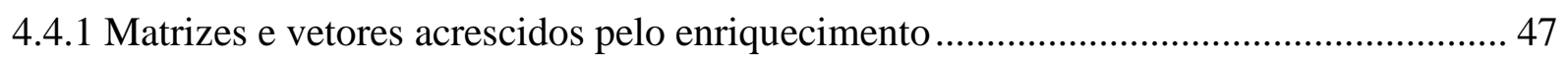

4.4.2 Sistema resolvente da formulação com enriquecimento ............................................... 48

4.4.3 Montagem do sistema resolvente global da formulação com enriquecimento ............... 49

5 Método da Partição em formulação híbrida-Trefftz com enriquecimento seletivo aplicado a problemas com múltiplas fissuras em domínios bidimensionais..................... 52

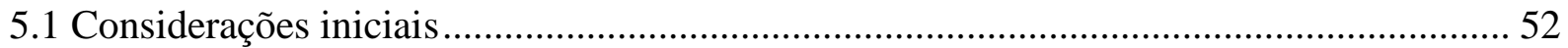

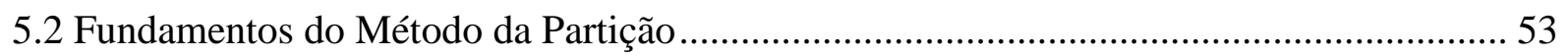

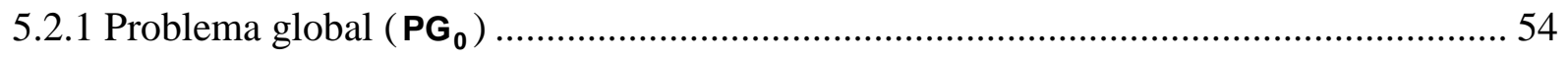

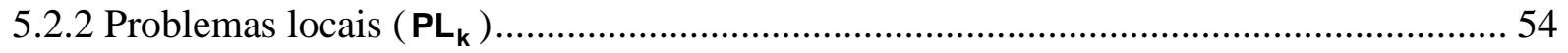

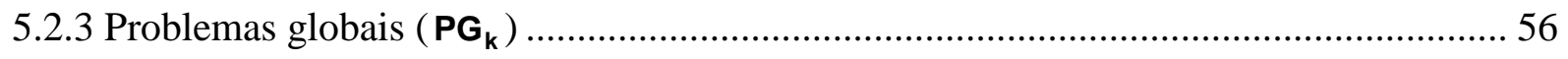

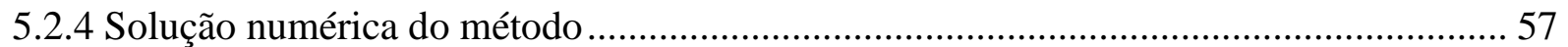

5.3 Equacionamento do Método da Partição em formulação híbrida-Trefftz com

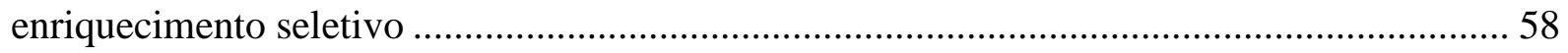

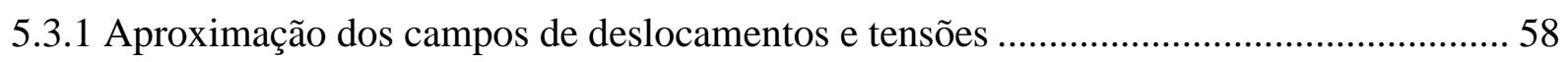

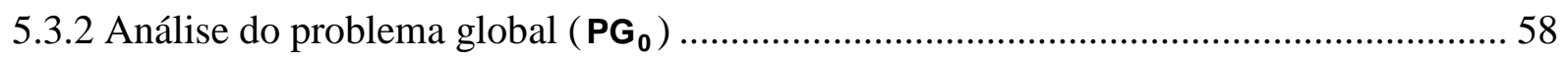

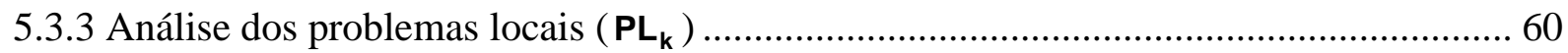

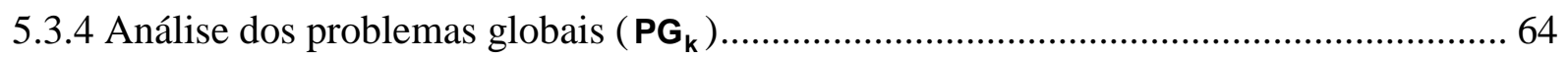

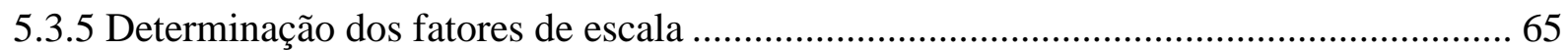

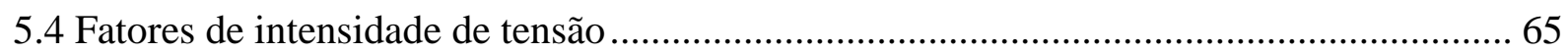

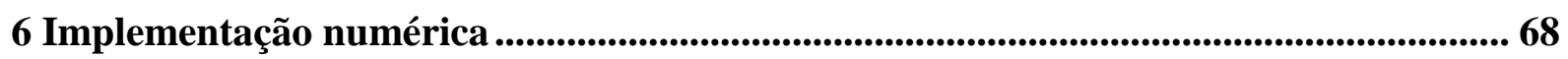

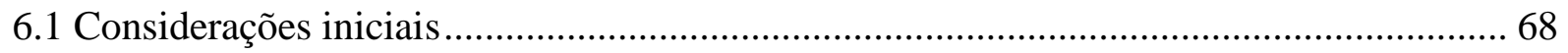

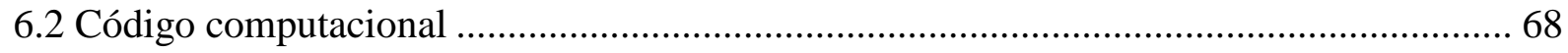

6.3 Sistemas de coordenadas empregados na implementação ............................................... 69

6.4 Implementação do elemento finito com domínio contínuo ............................................. 70 
6.4.1 Posição do centróide. 70

6.4.2 Mapeamento geométrico e determinação dos co-senos diretores .................................. 71

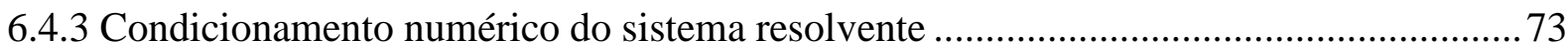

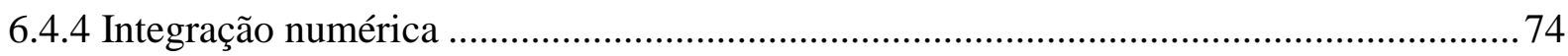

6.5 Implementação do elemento finito com orifício circular central .................................... 75

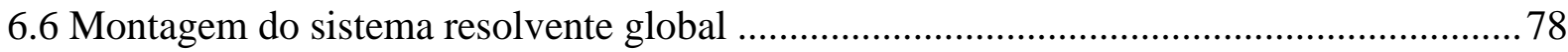

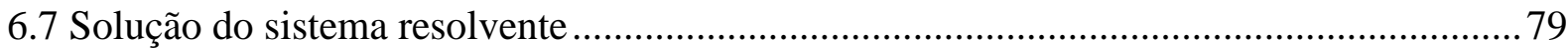

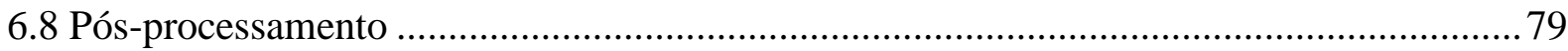

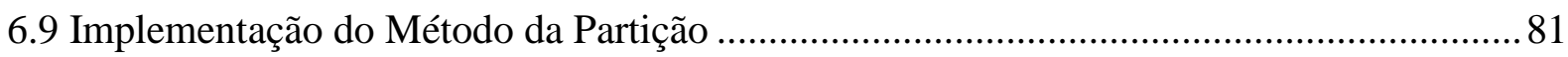

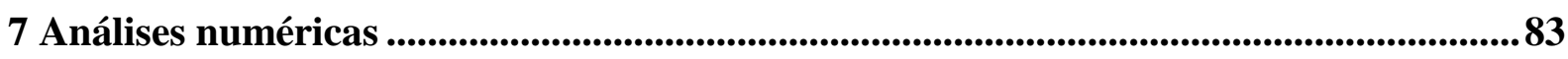

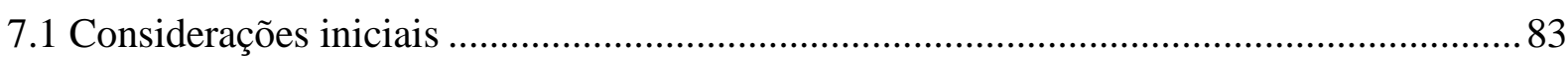

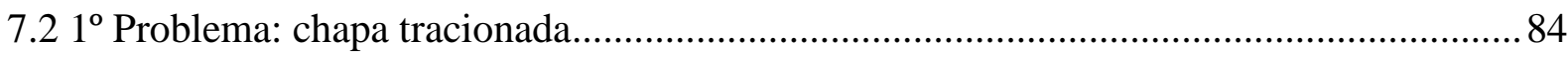

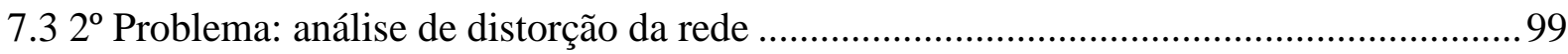

$7.43^{\circ}$ Problema: chapa com um orifício circular excêntrico ................................................ 104

$7.54^{\circ}$ Problema: chapa com fissura inserida no domínio....................................................... 113

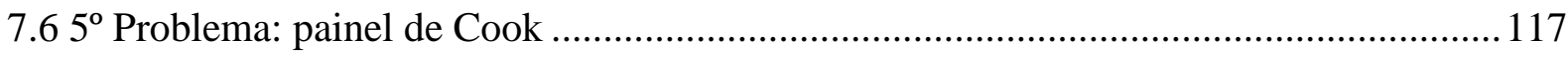

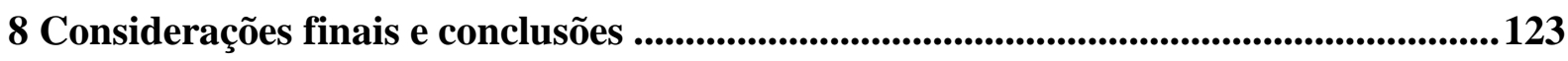

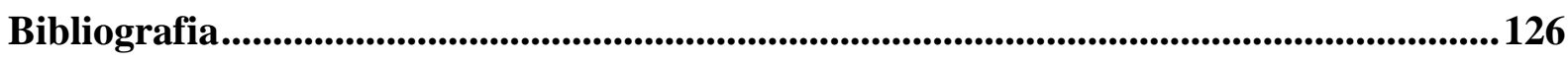

Apêndice A - Matrizes utilizadas na aproximação dos campos de deslocamentos e tensões em domínios contínuos: construção a partir da equação de Navier ..................... 130 Apêndice B - Matrizes utilizadas na aproximação dos campos de deslocamentos e tensões em domínios com orifício circular: construção a partir da equação de Navier 137 Apêndice C - Exemplo de montagem do sistema resolutivo .............................................. 140 Apêndice D - Exemplo de montagem do sistema resolutivo com enriquecimento ..........145 



\section{Introdução}

\subsection{Considerações gerais}

Problemas de interesse para a engenharia, normalmente relacionados à análise do comportamento de uma estrutura, ou material, e sua interação com o meio externo, podem ser formulados, teoricamente, a partir de princípios matemáticos e leis da Física. Se por um lado a complexidade dos fenômenos presentes na maioria desses problemas são entraves naturais à concepção de um modelo matemático minucioso, por outro, a introdução de certas hipóteses simplificadoras permite construir uma idealização suficientemente precisa conforme a qualidade da resposta pretendida. Neste contexto, cada problema idealizado passa a ser regido por um modelo matemático capaz de representar, à luz das hipóteses admitidas, os fenômenos observados.

A idealização mediante um modelo matemático é geralmente realizada por um conjunto de equações, sejam elas diferenciais, integrais ou algébricas, as quais, concomitantemente, devem satisfazer a condições de contorno específicas do problema analisado. Um problema de valor de contorno (PVC), típico do capítulo da elasto-estática na Mecânica dos Sólidos e das Estruturas, compõe-se de uma ou mais equações diferenciais envolvendo variáveis representativas dos campos de deslocamento, deformação e tensão, e que combinam restrições constitutivas do material, de equilíbrio e de compatibilidade. As condições de contorno, sejam em forças ou em deslocamentos, completam o PVC para um sólido ou estrutura.

A solução analítica de um PVC é por vezes laboriosa, restringindo-se a problemas relativamente simples, caracterizados por um número reduzido de variáveis, equações triviais e poucas condições de contorno. Inserido neste contexto, os métodos numéricos configuramse como alternativas interessantes.

Os métodos numéricos podem ser entendidos como técnicas sistemáticas de geração de respostas aproximadas de um dado problema. Apesar de suas origens estarem associadas ainda ao século XVIII, em aplicações numéricas do cálculo diferencial e integral, suas potencialidades permaneceram latentes durante séculos, em virtude do envolvimento de 
operações algébricas muitas vezes laboriosas, particularmente quando da resolução de problemas mais complexos. Esta situação modificou-se somente a partir do século XX com o advento de recursos computacionais, os quais viabilizaram a otimização de diversas operações de cálculo, sobretudo o matricial.

Dentre os diversos métodos numéricos, o Método dos Elementos Finitos (MEF) é, atualmente, um dos mais difundidos, tanto no meio técnico quanto no científico, com aplicações nas mais diversas áreas. A formulação clássica do MEF, também conhecida por "formulação em deslocamentos", é a mais utilizada, tendo sido concebida, originalmente, para a análise de problemas no âmbito da Mecânica das Estruturas.

$\mathrm{Na}$ formulação em deslocamentos apenas o campo de deslocamentos no domínio do elemento é aproximado diretamente. Os outros campos, de deformações e tensões, são obtidos indiretamente, mediante equações representativas das restrições de compatibilidade e constitutiva. O êxito desta formulação caracteriza-se por sua simplicidade conceitual e pela facilidade de implementação, propiciando sua incorporação de maneira ostensiva em diversos códigos computacionais comerciais.

Entretanto, a forma convencional do MEF apresenta certas limitações, dentre as quais, citam-se:

- Perda de precisão nas aproximações indiretas dos campos de tensões e deformações. As equações utilizadas na obtenção dos referidos campos envolvem derivações e consequentemente, implicam em redução da ordem da aproximação.

- Comprometimento da solução do problema, quando se empregam elementos excessivamente distorcidos.

- Em problemas contendo singularidades (problemas que envolvem elevados gradientes de tensões) uma resposta satisfatória só é obtida mediante uma discretização excessiva das zonas de interesse. Em situações de forte localização dos gradientes, muitos procedimentos automáticos de geração de redes acabam por não se restringir somente à região de singularidade, ocasionando refinamentos desnecessários em outras zonas do domínio, ou mesmo elementos excessivamente distorcidos.

- No caso de placas, cascas e sólidos tridimensionais, em virtude de restrições impostas no modelo teórico, observa-se a ocorrência do fenômeno de travamento, ou "locking", (BATHE, 1996), consistindo na forte redução da taxa de convergência da aproximação para a resposta correta. A superação de problemas deste tipo exige a formulação de 
elementos especiais ou mesmo discretização muito refinada, novamente aumentando excessivamente os custos computacionais.

Diante das limitações da forma convencional do MEF diferentes alternativas vêm sendo apresentadas. Dentre elas merecem destaque aquelas derivadas de formulações híbridomista, híbrida e híbrida-Trefftz, descritas, por exemplo, no trabalho de Freitas, Almeida e Pereira (1999), ou as que exploram o conceito de Partição da Unidade para o enriquecimento das aproximações, como o Método dos Elementos Finitos Generalizados (MEFG) (DUARTE; BABUŠKA e ODEN, 2000).

Uma característica comum entre as formulações híbrido-mista, híbrida e híbridaTrefftz é que em todas elas as aproximações são concebidas tanto no domínio quanto no contorno do elemento, daí o termo híbrido. Além disso, de cada uma podem ser propostas duas subformulações, a depender das hipóteses de continuidade admitidas a priori entre elementos. Elas são designadas formulação em deslocamento e formulação em tensão (FREITAS; ALMEIDA; PEREIRA, 1999). Nas formulações em deslocamento, o modelo é desenvolvido para propiciar uma solução cinematicamente admissível, isto é, que satisfaz localmente a compatibilidade entre deformações e deslocamentos no domínio do elemento e que garante a continuidade de deslocamentos nas fronteiras comuns de elementos contíguos. Por outro lado, nas formulações em tensão busca-se um modelo que seja estaticamente admissível, de forma a verificar o equilíbrio local no domínio do elemento e o equilíbrio na interface entre elementos adjacentes.

$\mathrm{Na}$ formulação híbrido-mista nenhuma restrição é imposta a priori sobre as funções aproximativas. A formulação é dita mista, pois dois campos de naturezas distintas são aproximados diretamente no domínio do elemento, sejam de tensões e deformações ou de tensões e deslocamentos. No contorno do elemento aproxima-se, de forma independente dos anteriores, o campo de tensões na formulação híbrido-mista de deslocamento ou o de deslocamentos na formulação híbrido-mista de tensão.

A formulação híbrida é um caso particular da formulação híbrido-mista. Na formulação híbrida de tensão, as funções aproximativas de tensões devem satisfazer a priori a equação de equilíbrio do problema. Em virtude desta restrição, demonstra-se que a aproximação de deformações ou deslocamentos no domínio torna-se redundante. Este modelo aproxima, então, dois campos independentes: o de tensões no domínio e o de deslocamentos no contorno. Por outro lado, na formulação híbrida de deslocamento a restrição a ser previamente atendida é a equação de compatibilidade. Nesta situação, a aproximação de tensão no domínio é que se torna redundante e, desta forma, os campos aproximados 
independentemente são de deslocamentos no domínio e de tensões no contorno.

Por sua vez, a formulação híbrida-Trefftz, foco principal deste trabalho, é a mais restritiva. As funções aproximativas não devem satisfazer apenas a condição de equilíbrio ou a de compatibilidade, mas a própria equação diferencial governativa do problema. Tanto na formulação em deslocamento quanto na de tensão, em virtude da restrição imposta sobre as funções aproximativas, o campo diretamente aproximado no domínio é o de tensões. No contorno aproxima-se o campo de tensões na formulação híbrida-Trefftz de deslocamento ou o campo de deslocamentos na formulação híbrida-Trefftz de tensão.

A formulação híbrida-Trefftz baseia-se no Método de Trefftz, o qual, em essência, consiste na aproximação das grandezas de interesse de um determinado problema, mediante o uso adequado das próprias soluções analíticas de sua equação regente como funções de aproximação. Por se tratar de um método integrante da formulação estudada neste trabalho será realizada uma abordagem mais ampla sobre o tema no capítulo 2.

Ainda no tocante à formulação híbrida-Trefftz, verificam-se algumas vantagens em comparação ao MEF convencional ou mesmo ao Método dos Elementos de Contorno (MEC). Ao contrário do MEF, as integrações são realizadas no contorno dos elementos, facilitando a implementação de elementos com formas poligonais arbitrárias ou curvas quaisquer. Além disto, o uso de funções aproximativas que satisfazem à equação governativa do problema evita a introdução de equações integrais singulares e, assim, dispensa a construção de soluções fundamentais como ocorre no MEC (JIROUSEK; WRÓBLEWSKI, 1996).

Segundo Qin (2005) uma outra potencialidade da referida formulação surge em problemas singulares, tais como regiões próximas a cavidades, pontas de fissuras e carregamentos, aonde o uso de funções especiais, de soluções analíticas locais, geram ótimas aproximações, sem o excessivo refinamento da rede, como ocorre no MEF convencional.

À parte as formulações híbridas citadas, o MEFG também tem se apresentado como uma alternativa interessante, em termos numéricos, para superar as limitações inerentes à forma convencional do MEF. O MEFG combina aspectos do MEF convencional com recursos dos métodos sem rede, particularmente quanto ao enriquecimento da base de funções de aproximação. Dentre as potencialidades do referido método citam-se: a robustez numérica, a possibilidade de se priorizar regiões de interesse no domínio estrutural, mediante o enriquecimento por zonas de influência, e a versatilidade de enriquecimento com diferentes tipos de funções. As técnicas de enriquecimento do MEFG serão descritas mais detalhadamente no capítulo 4.

Destacam-se, finalmente, em contraposição à forma convencional do MEF, 
proposições mais recentes, como as que combinam aspectos das formulações híbridas com as técnicas de enriquecimento preconizadas pelo MEFG. Neste contexto, citam-se o trabalho de Pimenta, Proença e Freitas (2002), no qual são introduzidas técnicas de enriquecimento nodal na formulação híbrido-mista de tensão. Na extensão desse trabalho, Góis (2004) amplia as possibilidades de enriquecimento da formulação híbrido-mista de tensão, mediante o emprego, entre outras, de funções que descrevem campos auto-equilibrados de tensões, funções trigonométricas e funções solução da Mecânica da Fratura Elástico-linear.

É importante ressaltar que todas as formulações não-convencionais foram concebidas visando superar algumas das limitações do MEF convencional. A formulação híbrida-Trefftz aplica-se com vantagens, sobretudo, na solução de problemas da Elasticidade Linear envolvendo singularidades.

Perante as considerações anteriores, vislumbram-se inúmeras possibilidades de pesquisas no âmbito das formulações não-convencionais do MEF.

\subsection{Objetivos}

$\mathrm{Na}$ esteira das pesquisas iniciadas em Pimenta, Proença e Freitas (2002) e Góis (2004), este trabalho visa ampliar às possibilidades de refinamento das formulações nãoconvencionais em elementos finitos, particularmente introduzindo aspectos combinados do MEFG e do clássico refino-p na formulação híbrida-Trefftz de tensão para a Elasticidade Bidimensional. Tal concepção é uma alternativa aos processos exclusivos p-adaptativos, os quais já foram explorados em elementos híbridos-Trefftz de grande destaque na literatura, como por exemplo, o HTD ("hybrid Trefftz displacement”), descrito por Jirouseck e Wróblewski (1996), e o HTS ("hybrid Trefftz stress"), apresentado por Freitas (1998).

O objetivo principal é desenvolver uma formulação que contemple o enriquecimento seletivo no domínio estrutural, sendo possível priorizar regiões de interesse. Por um lado, pretende-se avaliar o desempenho de três bases aproximativas adotadas para o enriquecimento dos deslocamentos no contorno dos elementos: base polinomial com refinamento hierárquico, polinomial com refinamento não-hierárquico e não-polinomial envolvendo funções trigonométricas. Por outro lado, aspira-se averiguar a influência do número de termos tomados na aproximação do campo de tensões no domínio do elemento, cujas funções de aproximação derivam da solução diferencial governativa do problema, sejam em elementos 
com domínio contínuo ou com orifício circular. Para avaliar o desempenho da formulação proposta serão realizadas análises de convergência de algumas grandezas de interesse. Os valores aproximados serão confrontados com valores de referência, sejam analíticos, obtidos via o MEF convencional ou aproximados por outras formulações encontradas na literatura.

Um objetivo complementar é a aplicação da formulação híbrida-Trefftz com enriquecimento seletivo à simulação numérica de problemas de fraturamento modelados segundo o Método da Partição (BABUŠKA; ANDERSSON, 2005). Particularmente, avalia-se a eficiência da alternativa numérica desenvolvida na resolução do problema de uma fissura inserida num domínio plano. Nesta avaliação será analisada a influência tanto do número de termos da série de carregamentos fictícios, inerente ao Método da Partição, quanto do enriquecimento seletivo, na extração do fator de intensidade de tensão.

\subsection{Estrutura da dissertação}

A estrutura da dissertação é composta de oito capítulos relacionados a tópicos específicos, conforme exposto no que se segue:

- No capítulo 1, inicialmente, são feitas as considerações gerais do trabalho, com o intuito de contextualizar a formulação estudada no âmbito dos métodos numéricos e justificar a relevância da pesquisa proposta. Em seguida, expõem-se os objetivos pretendidos da pesquisa. Finalmente, são feitas considerações sobre a organização de todo o texto da dissertação.

- O capítulo 2 foi concebido objetivando-se esclarecer os fundamentos do Método de Trefftz, particularmente na análise de problemas bidimensionais da Elasticidade. Após uma breve exposição de alguns conceitos básicos da teoria da Elasticidade, são apresentados os procedimentos inerentes à obtenção das funções aproximativas que satisfazem à equação de Navier, a qual rege o modelo elástico-linear.

- No capítulo 3 apresenta-se a formulação híbrida-Trefftz de forma simplificada, idealizada por aproximações lineares no contorno do elemento. Inicialmente, são definidas as aproximações do campo de tensões no domínio do elemento e de deslocamentos em seu contorno. Em seguida, equaciona-se a formulação a partir de certos princípios variacionais. Por fim, são feitas algumas considerações no tocante, tanto à solução do sistema resolutivo para o elemento, quanto à montagem e solução do sistema global. 
- No capítulo 4 estende-se a formulação apresentada no capítulo 3 mediante o enriquecimento seletivo. São apresentadas as bases de aproximação enriquecidas exploradas no trabalho e as mudanças inseridas em virtude do enriquecimento em todas as etapas da formulação do capítulo 3.

- No capítulo 5 aborda-se o Método da Partição em formulação híbrida-Trefftz com enriquecimento seletivo. Após a apresentação dos fundamentos do referido método, apresenta-se o seu equacionamento quando do emprego da formulação estudada neste trabalho.

- No capítulo 6 apresentam-se os recursos numéricos utilizados na implementação do programa desenvolvido.

- No capítulo 7 são relatadas as análises numéricas realizadas em alguns problemas peculiares, incluindo comentários acerca das respostas obtidas.

- No capítulo 8 são apresentadas as considerações finais e conclusões. 


\section{Campos aproximados em problemas bidimensionais da Elasticidade: concepção baseada no Método de Trefftz}

\subsection{Considerações iniciais}

A formulação clássica do Método de Trefftz foi proposta para analisar problemas de potencial em domínios bidimensionais. A idéia inovadora apresentada por Trefftz ${ }^{1}$ (1926 apud FERNANDEZ, 1998) consistia na adoção de funções aproximativas que satisfaziam a priori a equação governativa do problema.

Percebeu-se que o conceito primordial do Método de Trefftz, no tocante às funções aproximativas, poderia ser agregado a qualquer método numérico, particularmente na análise de problemas cuja solução da equação regente fosse factível. Entretanto, a indisponibilidade de recursos computacionais aliada à incipiência dos métodos numéricos inviabilizaria sua utilização por algumas décadas.

As aplicações pioneiras baseadas no Método de Trefftz em métodos numéricos foram realizadas via MEF. Nelas, as funções aproximativas que satisfaziam à equação regente do problema eram utilizadas em apenas parte do domínio, enquanto nas partes remanescentes a aproximação era obtida pela discretização convencional. Por exemplo, Willians ${ }^{2}$ (1952 apud JIROUSEK; WRÓBLEWSK, 1996) aplicou este procedimento para cantos reentrantes de placas, enquanto $\operatorname{Stein}^{3}(1972$ apud JIROUSEK; WRÓBLEWSK, 1996) executou o mesmo procedimento para uma região retangular pertencente a uma casca.

Posteriormente, Jirouseck (1978) apresentou quatro formulações de elementos finitos híbridos-Trefftz, diretamente aplicáveis à Mecânica dos Sólidos e das Estruturas. Nelas o atendimento à equação governativa passou abranger todo o domínio do problema. Desde então, inúmeras aplicações têm sido realizadas com sucesso em diversas áreas.

1 TREFFTZ, E. Ein Gegenstück zum Ritzschen Verfharen (1926). Procedures 2nd International Congress of Applied Mechanics, Zurich, p. 131-137.

2 WILLIANS, M. (1952). Surface stress singularities resulting from various boundary conditions in angular corners of plates in extension. J. Applied Mechanics, v.19, p. 526-528.

${ }^{3}$ STEIN, E. (1973). Die Kombination des modifizierten Trefftzschen Verfahrens mit der Methode der Finiten Elemente. Finite Element in der Statik (K.Buch, D. Scharpf, E.stein, and W. Wunderlich, eds.), p.172185. 
Nesse âmbito, citam-se as aplicações em problemas de Elasticidade (JIROUSEK; VENKATESH, 1992), placas de espessura moderada de Reisner-Mindlin (JIN; QIN, 1995), placas espessas (PETROLITO, 1996), Mecânica dos Sólidos Axi-simétricos (WRÓBLEWSKI; ZIELINSK; JIROUSEK, 1992), cascas (VÖRÖS; JIROUSECK, 1991), problemas elastodinâmicos (FREITAS, 1997), análise de condução de calor (JIROUSEK; QIN, 1996), placas sob não-linearidade geométrica (QIN, 1996), não-linearidade física em problemas tridimensionais da Elasticidade (BUSSAMRA; PIMENTA; FREITAS, 2001), materiais piezelétricos (QIN, 2003), entre outros.

É importante ressaltar que as formulações baseadas no Método de Trefftz não se restringem ao MEF. Neste contexto, referenciam-se os trabalhos de Fernandez (1998), onde se apresentam formulações dos Métodos dos Resíduos Ponderados, em particular enfatizando aplicações via o Método da Colocação de Forma Indireta, o de Leitão (2001), no qual se realiza uma análise comparativa entre o Método de Galerkin e o Método da Colocação em problemas bidimensionais da Elasticidade, o de Díaz, Herreira e Yates (2002), onde se trata dos Métodos Indiretos de Colocação e o de Chang et al. (2002), em que se aborda uma maneira direta da aplicação do Método de Trefftz como um método de contorno em problemas de membranas com vibrações livres.

Em todos os trabalhos citados, constata-se que o estabelecimento das funções aproximativas, baseadas no Método de Trefftz, foi uma condição preliminar na concepção das correspondentes formulações propostas.

Assim sendo, para os objetivos deste trabalho, torna-se imprescindível um bom entendimento das funções aproximativas concebidas pelo Método de Trefftz para a análise de problemas bidimensionais da Elasticidade. Desta forma, inicialmente, apresentam-se neste capítulo algumas relações básicas de Elasticidade, com intuito de apresentar a notação utilizada e, sobretudo, estabelecer a equação governativa do problema. Logo em seguida, são descritos os procedimentos relacionados à construção das aproximações dos campos de deslocamentos e tensões.

\subsection{Relações básicas em problemas de Elasticidade}

$\mathrm{Na}$ formulação de um PVC em Elasticidade, considera-se um corpo elástico em 
equilíbrio, composto de uma região interna, ou domínio aberto $\Omega$, e envolto por uma superfície regular, ou contorno $\Gamma$. Admite-se que em $\Omega$ atuam forças volúmicas, representadas pelo vetor resultante $\boldsymbol{b}$ e que em $\Gamma$ agem forças de superfície, representadas por $\overline{\boldsymbol{t}}$. Além das forças de superfície, deslocamentos $\overline{\boldsymbol{u}}$ podem ser impostos ou impedidos em parte do contorno. Assim, para efeito de equacionamento, o contorno $\Gamma$ passa a ser composto de duas regiões complementares, a saber, a fronteira estática $\Gamma_{\mathrm{t}}$ e a cinemática $\Gamma_{\mathrm{u}}$ onde, respectivamente, os valores de $\overline{\boldsymbol{t}}$ e $\overline{\boldsymbol{u}}$ são prescritos, conforme ilustrado na Figura 2.1.

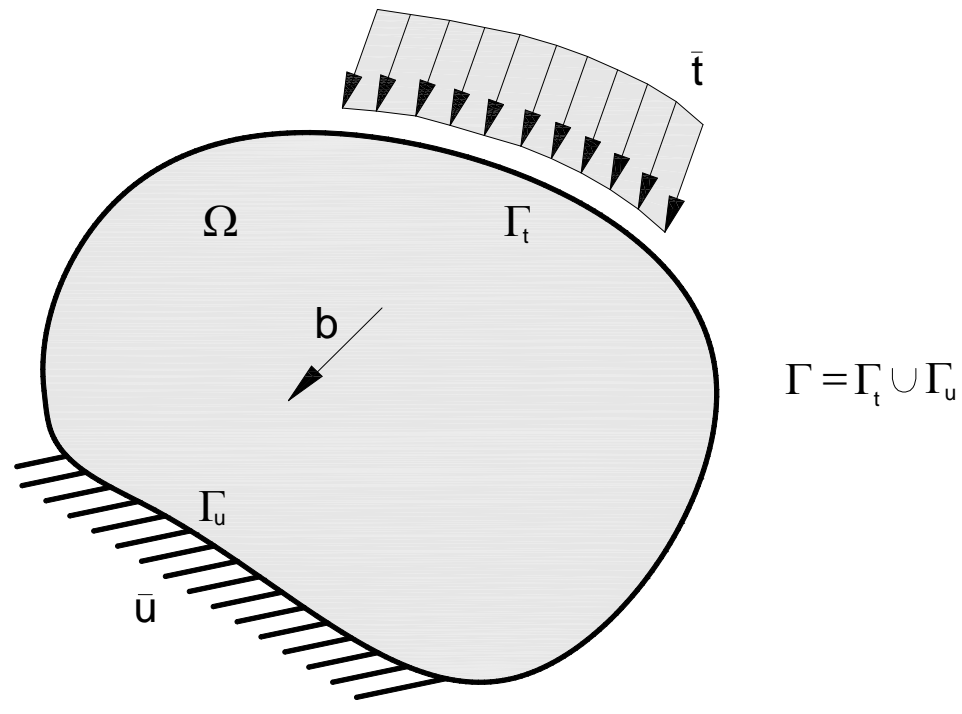

Figura 2.1. Representação de domínio, fronteiras e forças em um corpo elástico.

De acordo com as definições anteriores, observam-se, as seguintes relações entre os conjuntos $\Omega, \Gamma, \Gamma_{\mathrm{u}}$ e $\Gamma_{\mathrm{t}}$ :

- $\Omega \cap \Gamma=\varnothing$

- $\Gamma_{\mathrm{u}} \cup \Gamma_{\mathrm{t}}=\Gamma$

- $\Gamma_{\mathrm{u}} \cap \Gamma_{\mathrm{t}}=\varnothing$

Apresenta-se, a seguir, o conjunto de equações que governam o PVC para o estado plano de tensão (EPT). Todas as relações são válidas para materiais elásticos, isótropos e sob condições de linearidade física e geométrica. Os procedimentos e particularidades da teoria podem ser encontrados em obras clássicas como: Fung (1965), Timoshenko e Goodier (1980) e Valliappan (1981). As variáveis envolvidas serão especificadas a partir de dois sistemas de interesse: o cartesiano, de coordenadas $x$ e $y$, e o polar, de coordenadas $r$ e $\theta$. Na Figura 2.2 estão ilustrados estes sistemas e algumas grandezas a eles referidas, mas que ainda serão 
definidas.

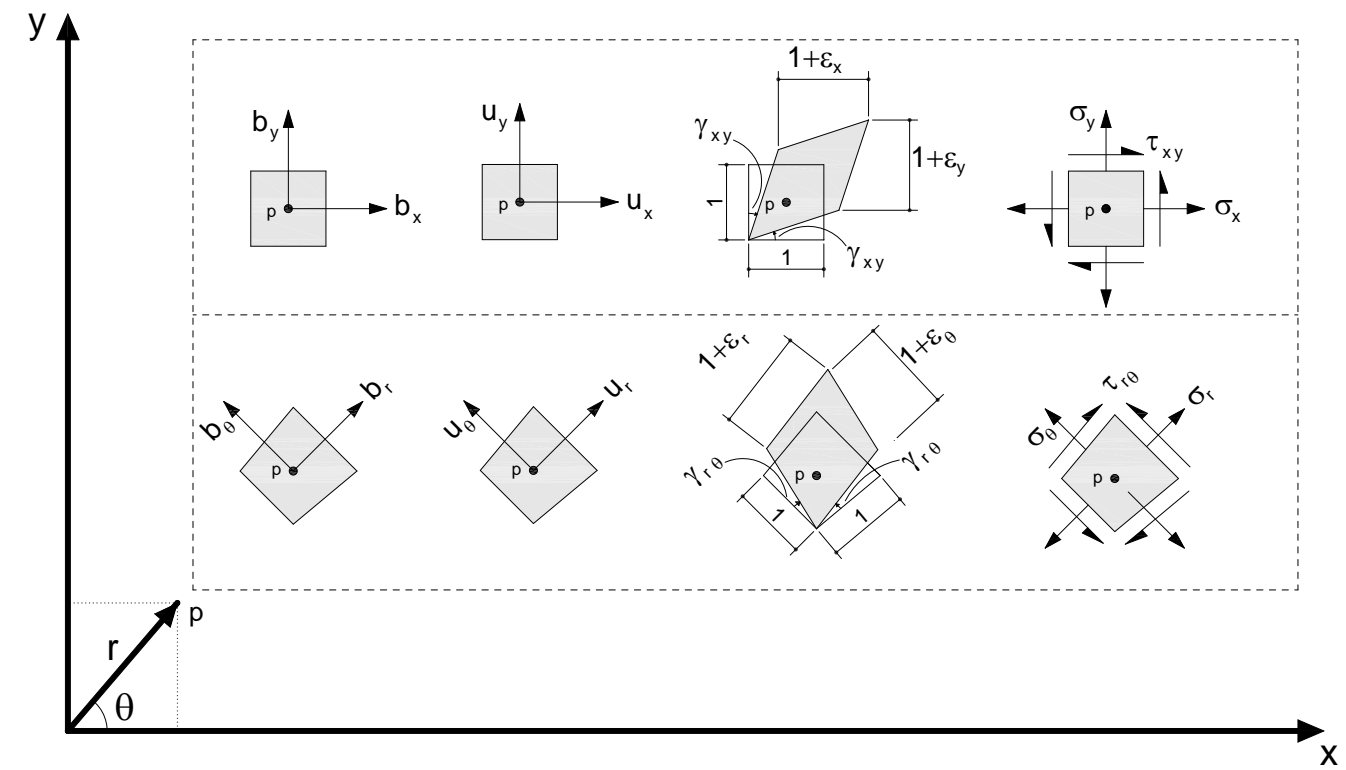

Figura 2.2. Sistemas de coordenadas e representação das componentes de forças volúmicas, deslocamentos, deformações e tensões num dado ponto.

\section{- Equação de equilíbrio}

Segundo o sistema de coordenadas cartesianas, tem-se:

$$
L_{c} \sigma_{c}+b_{c}=0, \text { em } \Omega
$$

onde $L_{c}$ é uma matriz formada por operadores diferenciais; $\sigma_{c}$ o vetor representativo do tensor das tensões e $\boldsymbol{b}_{\boldsymbol{c}}$ o vetor de forças volúmicas. Estas grandezas podem ser representadas por:

- $\quad \boldsymbol{L}_{c}=\left[\begin{array}{ccc}\partial / \partial \mathrm{x} & 0 & \partial / \partial \mathrm{y} \\ 0 & \partial / \partial \mathrm{y} & \partial / \partial \mathrm{x}\end{array}\right] ;$ sendo $\partial / \partial x$ e $\partial / \partial y$ operadores de derivadas parciais.

- $\boldsymbol{\sigma}_{c}{ }^{T}=\left\{\begin{array}{lll}\sigma_{\mathrm{x}} & \sigma_{\mathrm{y}} & \tau_{\mathrm{xy}}\end{array}\right\}$; sendo $\sigma_{\mathrm{x}}$ e $\sigma_{\mathrm{y}}$ componentes normais do tensor de tensões,

enquanto $\tau_{x y}$ é a componente cisalhante.

- $\quad \boldsymbol{b}_{c}{ }^{\top}=\left\{\begin{array}{ll}b_{x} & b_{y}\end{array}\right\}$; sendo $b_{\mathrm{x}}$ e $b_{\mathrm{y}}$ componentes das forças volúmicas.

Segundo o sistema de coordenadas polares, escreve-se a equação de equilíbrio na forma:

$$
M_{\sigma} \sigma_{p}+L_{p} \sigma_{p}+b_{p}=0, \text { em } \Omega
$$

onde $\boldsymbol{M}_{\boldsymbol{\sigma}}$ é uma matriz que depende da posição $r ; \boldsymbol{L}_{\boldsymbol{p}}$ é uma matriz formada por operadores diferenciais; $\sigma_{p}$ é o vetor que representa o tensor das tensões e $\boldsymbol{b}_{\boldsymbol{p}}$ o vetor de forças 
volúmicas. Estas grandezas admitem o aspecto:

- $\boldsymbol{M}_{\boldsymbol{\sigma}}=\left[\begin{array}{ccc}1 / r & -1 / r & 0 \\ 0 & 0 & 2 / r\end{array}\right]$; sendo r a posição de um ponto em relação à origem.

- $\quad \boldsymbol{L}_{\boldsymbol{p}}=\left[\begin{array}{ccc}\partial / \partial r & 0 & (1 / r) \cdot \partial / \partial \theta \\ 0 & (1 / r) \cdot \partial / \partial \theta & \partial / \partial r\end{array}\right]$; sendo $\partial / \partial r$ e $\partial / \partial \theta$ operadores de derivadas parciais.

- $\boldsymbol{\sigma}_{\boldsymbol{p}}{ }^{T}=\left\{\begin{array}{lll}\sigma_{\mathrm{r}} & \sigma_{\theta} & \tau_{\mathrm{r} \theta}\end{array}\right\}$; sendo $\sigma_{\mathrm{r}}$ e $\sigma_{\theta}$ componentes normais do tensor de tensões, enquanto $\tau_{\mathrm{r} \theta}$ é a componente cisalhante.

- $\boldsymbol{b}_{\boldsymbol{p}}{ }^{T}=\left\{\begin{array}{ll}b_{r} & b_{\theta}\end{array}\right\}$; sendo $b_{r}$ e $b_{\theta}$ componentes das forças volúmicas.

\section{- Equação de compatibilidade}

Segundo o sistema de coordenadas cartesianas, tem-se:

$$
\boldsymbol{\varepsilon}_{c}=\boldsymbol{L}_{c}{ }^{T} \boldsymbol{u}_{c}, \text { em } \Omega
$$

onde $\varepsilon_{c}$ é o vetor que representa o tensor das deformações e $\boldsymbol{u}_{c}$ o vetor do campo de deslocamentos. As seguintes representações são possíveis:

- $\boldsymbol{\varepsilon}_{c}{ }^{T}=\left\{\begin{array}{lll}\varepsilon_{\mathrm{x}} & \varepsilon_{\mathrm{y}} & \gamma_{\mathrm{xy}}\end{array}\right\}$; onde $\varepsilon_{\mathrm{x}}$ e $\varepsilon_{\mathrm{y}}$ são as componentes da deformação linear específica e $\gamma_{x y}$ é a distorção angular.

- $u_{c}{ }^{T}=\left\{u_{x} u_{y}\right\} ;$ sendo $u_{x}$ e $u_{y}$ componentes do campo de deslocamentos.

Já em coordenadas polares, escreve-se:

$$
\varepsilon_{p}=M_{\varepsilon} u_{p}+L_{p}{ }^{T} u_{p}, \text { em } \Omega
$$

onde $\varepsilon_{\mathrm{p}}$ representa o tensor das deformações, $\boldsymbol{u}_{\boldsymbol{p}}$ o vetor do campo de deslocamentos e $\boldsymbol{M}_{\boldsymbol{\varepsilon}}$ uma matriz que depende da posição $r$. Estas grandezas admitem o aspecto:

- $\boldsymbol{\varepsilon}_{\boldsymbol{p}}{ }^{T}=\left\{\begin{array}{lll}\varepsilon_{\mathrm{r}} & \varepsilon_{\theta} & \gamma_{\mathrm{r} \theta}\end{array}\right\} ;$ onde $\varepsilon_{\mathrm{r}}$ e $\varepsilon_{\theta}$ são as componentes da deformação linear específica e $\gamma_{\mathrm{r} \theta}$ é a distorção angular.

- $\boldsymbol{u}_{\boldsymbol{p}}{ }^{T}=\left\{\begin{array}{ll}u_{\mathrm{r}} & \mathrm{u}_{\theta}\end{array}\right\}$; sendo $\mathrm{u}_{\mathrm{r}}$ e $\mathrm{u}_{\theta}$ componentes do campo de deslocamentos.

- $\boldsymbol{M}_{\boldsymbol{\varepsilon}}=\left[\begin{array}{cc}0 & 0 \\ 1 / r & 0 \\ 0 & -1 / r\end{array}\right]$; sendo $r$ a posição de um dado ponto.

\section{- Relação Constitutiva}


Segundo o sistema de coordenadas cartesianas, tem-se:

$$
\sigma_{c}=D \varepsilon_{c} \text { ou } \varepsilon_{c}=f \sigma_{c} \text { em } \Omega
$$

enquanto que em coordenadas polares, escreve-se:

$$
\sigma_{p}=D \varepsilon_{p} \text { ou } \varepsilon_{p}=f \sigma_{p} \text { em } \Omega
$$

onde $\boldsymbol{D}$ é o tensor constitutivo de rigidez e $\boldsymbol{f}$ o de flexibilidade. Tem-se a seguinte representação em forma matricial:

- $\boldsymbol{D}=\frac{E}{1-v^{2}}\left[\begin{array}{ccc}1 & v & 0 \\ v & 1 & 0 \\ 0 & 0 & (1-v) / 2\end{array}\right] ; \boldsymbol{f}=\frac{1}{E}\left[\begin{array}{ccc}1 & -v & 0 \\ -v & 1 & 0 \\ 0 & 0 & 2(1+v)\end{array}\right]$, sendo $E$ o módulo de Young e

$v$ o coeficiente de Poisson.

\section{- Transformação de coordenadas e condições de contorno}

A transformação de coordenadas entre o sistema cartesiano e o polar leva às relações de interesse:

$$
\begin{aligned}
& \boldsymbol{u}_{c}=\boldsymbol{R}_{u \theta} \boldsymbol{u}_{p} \\
& \boldsymbol{\sigma}_{c}=\boldsymbol{R}_{\sigma \theta} \boldsymbol{\sigma}_{p}
\end{aligned}
$$

onde as matrizes $\boldsymbol{R}_{u \theta}$ e $\boldsymbol{R}_{\sigma \theta}$ são matrizes de rotação que dependem do ângulo $\theta$ (vide Fig.2.2):

$$
\text { - } \quad \boldsymbol{R}_{u \theta}=\left[\begin{array}{cc}
\cos \theta & -\operatorname{sen} \theta \\
\operatorname{sen} \theta & \cos \theta
\end{array}\right] ; \quad \boldsymbol{R}_{\sigma \theta}=\left[\begin{array}{ccc}
\cos ^{2} \theta & \operatorname{sen}^{2} \theta & -2 \operatorname{sen} \theta \cos \theta \\
\operatorname{sen}^{2} \theta & \cos ^{2} \theta & 2 \operatorname{sen} \theta \cos \theta \\
\operatorname{sen} \theta \cos \theta & -\operatorname{sen} \theta \cos \theta & \cos ^{2} \theta-\operatorname{sen}^{2} \theta
\end{array}\right] ; \quad \text { com } \quad \theta
$$

positivo em sentido anti-horário.

As condições de contorno nas fronteiras cinemática e estática, equacionadas geralmente para o sistema cartesiano, são expressas por:

$$
\begin{aligned}
& \boldsymbol{u}_{\boldsymbol{c}}=\overline{\boldsymbol{u}}_{\boldsymbol{c}}, \text { em } \Gamma_{\mathrm{u}} \\
& \boldsymbol{t}_{\boldsymbol{c}}=\boldsymbol{A} \boldsymbol{\sigma}_{\boldsymbol{c}}=\overline{\boldsymbol{t}}_{\boldsymbol{c}}, \text { em } \Gamma_{\mathrm{t}}
\end{aligned}
$$

onde $\overline{\boldsymbol{u}}_{c}$ é o vetor de deslocamentos prescritos em $\Gamma_{\mathrm{u}}, \boldsymbol{A}$ uma matriz formada pelas componentes de um vetor unitário normal à $\Gamma_{\mathrm{t}}, \boldsymbol{t}_{c}$ o vetor das tensões em $\Gamma_{\mathrm{t}}$ e $\overline{\boldsymbol{t}}_{c}$ o vetor de forças de superfície prescritas em $\Gamma_{t}$. As seguintes representações são possíveis:

- $\bar{u}_{c}^{T}=\left\{\begin{array}{ll}\bar{u}_{\mathrm{x}} & \overline{\mathrm{u}}_{\mathrm{y}}\end{array}\right\} ;$ sendo $\overline{\mathrm{u}}_{\mathrm{x}}$ e $\overline{\mathrm{u}}_{\mathrm{y}}$ componentes dos deslocamentos prescritos.

- $\quad \mathbf{t}_{\mathbf{c}}{ }^{\top}=\left\{\begin{array}{ll}\mathrm{t}_{\mathrm{x}} & \mathrm{t}_{\mathrm{y}}\end{array}\right\}$; sendo $\mathrm{t}_{\mathrm{x}}$ e $\mathrm{t}_{\mathrm{y}}$ componentes das tensões em $\Gamma_{\mathrm{t}}$. 
- $\quad \overline{\boldsymbol{t}}_{c}{ }^{T}=\left\{\begin{array}{ll}\overline{\mathrm{t}}_{x} & \overline{\mathrm{t}}_{y}\end{array}\right\}$; sendo $\bar{t}_{x}$ e $\bar{t}_{y}$ componentes das forças de superfície prescritas.

- $\boldsymbol{A}=\left[\begin{array}{ccc}n_{x} & 0 & n_{y} \\ 0 & n_{y} & n_{x}\end{array}\right]$; sendo $n_{x}$ e $n_{y}$ componentes de um vetor unitário $\boldsymbol{n}$ normal à superfície $\Gamma_{\mathrm{t}}$.

A Eq. (2.10) estabelece que as forças de superfície prescritas que agem num ponto qualquer do contorno devem estar em equilíbrio com o estado de tensões ao redor daquele ponto (teorema de Cauchy).

\section{- Equação de Navier}

A combinação das Eqs. (2.1), (2.3) e (2.5) ou (2.2), (2.4) e (2.6) pode ser representada genericamente por:

$$
\mathfrak{R}(\boldsymbol{u})=-\boldsymbol{b} \text { (Equação governativa do problema em } \Omega \text { ) }
$$

onde $\boldsymbol{u}$ e $\boldsymbol{b}$ representam, respectivamente, o campo de deslocamentos e o vetor de forças volúmicas e $\Re$ é um operador matricial.

Segundo o sistema de coordenadas cartesianas, onde $\boldsymbol{u}=\boldsymbol{u}_{\boldsymbol{c}}$ e $\boldsymbol{b}=\boldsymbol{b}_{\boldsymbol{c}}$, a Eq. (2.11) adquire o aspecto:

$$
\Re\left(u_{c}\right)=L_{c}\left[D L_{c}{ }^{T}\left(u_{c}\right)\right]=-b_{c}, \text { em } \Omega
$$

cuja expansão leva ao conjunto de equações diferenciais:

$$
\left\{\begin{array}{l}
\frac{\partial^{2} u_{x}}{\partial x^{2}}+\frac{1-v}{2} \frac{\partial^{2} u_{x}}{\partial y^{2}}+\frac{1+v}{2} \frac{\partial^{2} u_{y}}{\partial x \partial y}=-\frac{b_{x}}{\lambda_{c}} \\
\frac{\partial^{2} u_{y}}{\partial y^{2}}+\frac{1-v}{2} \frac{\partial^{2} u_{y}}{\partial x^{2}}+\frac{1+v}{2} \frac{\partial^{2} u_{x}}{\partial x \partial y}=-\frac{b_{y}}{\lambda_{c}}
\end{array} \quad, \text { em } \Omega\right.
$$

onde $\lambda_{c}=\frac{E}{1-v^{2}}$.

Por outro lado, quando do emprego do sistema de coordenadas polares, onde $\mathbf{u}=\mathbf{u}_{\mathrm{p}} \mathrm{e}$ $\mathbf{b}=\mathbf{b}_{\mathbf{p}}$, a Eq. (2.11) passa a ser expressa por:

$$
\mathfrak{R}\left(u_{p}\right)=M_{\sigma} D M_{\varepsilon} u_{p}+M_{\sigma} D L_{p}{ }^{T}\left(u_{p}\right)+L_{p}\left(D M_{\varepsilon} u_{p}\right)+L_{p}\left[D L_{p}{ }^{T}\left(u_{p}\right)\right]=-b_{p}, \text { em } \Omega
$$

cuja expansão leva ao conjunto de equações diferenciais:

$$
\left\{\begin{array}{c}
2 \frac{\partial^{2} u_{r}}{\partial r^{2}}+\frac{1-v}{r^{2}} \frac{\partial^{2} u_{r}}{\partial \theta^{2}}+\frac{1+v}{r} \frac{\partial^{2} u_{\theta}}{\partial r \partial \theta}+\frac{2}{r} \frac{\partial u_{r}}{\partial r}-\frac{3-v}{r^{2}} \frac{\partial u_{\theta}}{\partial \theta}-\frac{2}{r^{2}} u_{r}=-\frac{b_{r}}{\lambda_{p}} \\
\frac{2}{r^{2}} \frac{\partial^{2} u_{\theta}}{\partial \theta^{2}}+(1-v) \frac{\partial^{2} u_{\theta}}{\partial r^{2}}+\frac{1+v}{r} \frac{\partial^{2} u_{r}}{\partial r \partial \theta}+\frac{1-v}{r} \frac{\partial u_{\theta}}{\partial r}+\frac{3-v}{r^{2}} \frac{\partial u_{r}}{\partial \theta}-\frac{1-v}{r^{2}} u_{\theta}=-\frac{b_{\theta}}{\lambda_{p}}
\end{array}, \text { em } \Omega\right.
$$


onde $\lambda_{p}=\frac{E}{2\left(1-v^{2}\right)}$.

A Eq. (2.11), conhecida por equação de Navier, governa o problema elástico. Esta equação acrescida das condições de contorno (Eqs. (2.9) e (2.10)) constituem a chamada 'forma forte' do PVC. Esta denominação decorre da exigência estabelecida pela Eq. (2.11), a qual deve ser satisfeita, ponto a ponto, em todo domínio $\Omega$.

Embora todo o equacionamento anterior (da Eq. (2.1) à Eq. (2.15)) tenha sido deduzido para o EPT, o mesmo também vale para o estado plano de deformação (EPD), desde que o módulo de Young $E$ e o coeficiente de Poisson $v$ sejam substituídos pelas constantes $E^{\prime}=E /\left(1-v^{2}\right)$ e $v^{\prime}=v /(1-v)$, respectivamente.

\subsection{Aproximação do campo de deslocamentos: construção a partir da equação de Navier}

Conforme já mencionado, o Método de Trefftz fundamenta-se na adoção de funções aproximativas que satisfazem $a$ priori a equação governativa do problema analisado. Por conseguinte, no caso do problema elástico linear, a equação de Navier constitui-se na restrição que as funções aproximativas utilizadas para aproximar as componentes do campo de deslocamentos devem atender.

Assim sendo, admite-se que o vetor solução geral da Eq. (2.11) seja composto de uma parcela particular $\hat{\mathbf{u}}$, outra homogênea $\boldsymbol{u}_{\boldsymbol{h}}$ e de uma terceira $\boldsymbol{u}_{M}$, que incorpora os movimentos de corpo rígido:

$$
\mathbf{u}=\hat{\mathbf{u}}+\boldsymbol{u}_{\boldsymbol{h}}+\boldsymbol{u}_{\boldsymbol{M}}, \text { em } \Omega
$$

A solução particular depende das características do problema considerado. O caso mais comum é aquele em que û depende exclusivamente das forças volúmicas, que se traduz na restrição:

$$
\Re(\hat{\mathbf{u}})=-\boldsymbol{b}, \text { em } \Omega
$$

onde $\hat{\boldsymbol{u}}^{T}=\left\{\begin{array}{ll}\hat{u}_{x} & \hat{u}_{y}\end{array}\right\}$, sendo $\hat{u}_{x}$ e $\hat{u}_{y}$ funções que em conjunto constituem a solução particular.

Além desta situação, revestem-se de interesse as regiões próximas a forças concentradas. Neste caso, pode-se desprezar o efeito das forças volúmicas e adotar a própria solução analítica da Elasticidade como solução particular, conforme realizado no trabalho de 
Jirouseck e Venkatesh (1992).

Por sua vez, a solução homogênea é obtida de:

$$
\mathfrak{R}\left(\boldsymbol{u}_{\boldsymbol{h}}\right)=\boldsymbol{0}, \text { em } \Omega
$$

A solução homogênea não é única, sendo possível aproximá-la por uma combinação linear de vetores, cada qual atendendo à restrição imposta pela Eq. (2.18). Consequentemente, explorando-se a notação matricial e indicial, escreve-se:

$$
\boldsymbol{u}_{\boldsymbol{h}}=\sum_{j=1}^{n_{C}} c_{j} \boldsymbol{N}_{\boldsymbol{j}}=\boldsymbol{N} \boldsymbol{c}, \operatorname{com} \mathfrak{R}\left(\boldsymbol{N}_{\boldsymbol{j}}\right)=\mathbf{0} \operatorname{em} \Omega
$$

Na Eq. (2.19), o vetor c é formado pelos parâmetros da combinação linear $c_{j}$, sendo representado por:

$$
\boldsymbol{c}^{T}=\left\{\begin{array}{lllll}
c_{1} & \cdots & c_{j} & \cdots & c_{n_{c}}
\end{array}\right\}
$$

Por sua vez, a matriz $\mathbf{N}$, constituída pelos vetores $\mathbf{N}_{\mathrm{j}}$, toma a forma:

$$
\text { - } \quad \boldsymbol{N}=\left[\begin{array}{lllll}
\boldsymbol{N}_{1} & \cdots & \boldsymbol{N}_{j} & \cdots & \boldsymbol{N}_{n_{c}}
\end{array}\right] ; \text { sendo } \boldsymbol{N}_{j}^{T}=\left\{\begin{array}{ll}
\boldsymbol{N}_{j x} & N_{j y}
\end{array}\right\} \text {, para } j=1, \cdots, n_{c} .
$$

Na representação anterior, cada vetor solução $\boldsymbol{N}_{\boldsymbol{j}}, j=1, \cdots, n_{c}$, é formado pelas funções de aproximação $N_{j x}$ e $N_{j y}$, as quais em conjunto constituem uma solução da Eq. (2.18).

Já a solução referente aos movimentos de corpo rígido inclui dois movimentos de translação e um de rotação, o que totaliza três vetores. A restrição a ser atendida é idêntica à da solução homogênea. Assim sendo, escreve-se:

$$
\boldsymbol{u}_{\boldsymbol{M}}=\sum_{j=1}^{3} \overline{\boldsymbol{c}}_{j} \overline{\boldsymbol{N}}_{\boldsymbol{j}}=\overline{\boldsymbol{N}} \overline{\boldsymbol{c}}, \operatorname{com} \mathfrak{R}\left(\overline{\boldsymbol{N}}_{\boldsymbol{j}}\right)=\mathbf{0} \text { em } \Omega
$$

onde a notação utilizada é análoga a da Eq. (2.19). Consequentemente, o vetor $\bar{c}$ e a matriz $\overline{\boldsymbol{N}}$ admitem o aspecto:

$$
\begin{aligned}
& \overline{\boldsymbol{c}}^{T}=\left\{\begin{array}{lll}
\bar{c}_{1} & \bar{c}_{2} & \bar{c}_{3}
\end{array}\right\} \\
& \text { - } \quad \overline{\boldsymbol{N}}=\left[\begin{array}{lll}
\overline{\boldsymbol{N}}_{1} & \overline{\boldsymbol{N}}_{2} & \overline{\boldsymbol{N}}_{3}
\end{array}\right] ; \text { sendo } \overline{\boldsymbol{N}}_{j}^{T}=\left\{\begin{array}{ll}
\bar{N}_{j x} & \bar{N}_{j y}
\end{array}\right\}, \text { para } j=1, \cdots, 3 .
\end{aligned}
$$

É importante salientar que a característica fundamental que distingue a solução homogênea da referente aos movimentos de corpo rígido relaciona-se aos vetores solução. Ao contrário dos vetores $\boldsymbol{N}_{j}$, os vetores $\overline{\boldsymbol{N}}_{j}$ possuem significado físico, ou seja, as funções de aproximação $\bar{N}_{j x}$ e $\bar{N}_{j y}$ em conjunto descrevem movimentos de corpo rígido, aos quais se associam tensores de tensões e deformações nulos. 
A partir das Eqs. (2.16), (2.19) e (2.20) a aproximação do campo de deslocamentos pode ser expressa por:

$$
\mathbf{u}=\hat{\mathbf{u}}+\boldsymbol{u}_{\boldsymbol{h}}+\boldsymbol{u}_{\boldsymbol{M}}=\hat{\mathbf{u}}+\sum_{j=1}^{n_{C}} c_{j} \boldsymbol{N}_{j}+\sum_{j=1}^{3} \bar{c}_{j} \overline{\boldsymbol{N}}_{\boldsymbol{j}}=\hat{\mathbf{u}}+\mathbf{N} \mathbf{c}+\overline{\boldsymbol{N}} \overline{\mathbf{c}}, \text { em } \Omega
$$

Na Eq. (2.21), os parâmetros $c_{j}$ e $\bar{c}_{j}$ constituem-se nas incógnitas da aproximação e, embora não possuam significado físico, serão doravante referidos por graus de liberdade. Tais parâmetros podem ser obtidos mediante um método numérico adequado, que incorpore as condições de contorno do problema. Segundo Jirousek e Wróblewski (1996), se as funções de aproximação adotadas possuem boas propriedades de representação, a solução dada pela Eq. (2.21) converge para a solução exata à medida que o número de termos tomados na aproximação aumenta.

Sem prejuízos para os objetivos deste trabalho, pode-se desprezar o efeito das forças volúmicas na construção da aproximação ditada pela Eq. (2.21). Por conseguinte, a solução particular torna-se trivial, isto é $\hat{\mathbf{u}}=\mathbf{0}$, e consequentemente a aproximação do campo de deslocamentos passa a ser representada por:

$$
\mathbf{u}=\mathbf{u}_{\boldsymbol{h}}+\boldsymbol{u}_{\boldsymbol{M}}=\sum_{j=1}^{n_{C}} \boldsymbol{c}_{j} \boldsymbol{N}_{\boldsymbol{j}}+\sum_{j=1}^{3} \overline{\boldsymbol{c}}_{j} \overline{\boldsymbol{N}}_{\boldsymbol{j}}=\mathbf{N} \mathbf{c}+\overline{\boldsymbol{N}} \overline{\boldsymbol{c}}, \text { em } \Omega
$$

Na construção das matrizes $\boldsymbol{N}$ e $\overline{\boldsymbol{N}}$ serão analisados domínios contínuos e domínios com orifício circular, como exposto nos dois próximos itens.

\subsubsection{Aproximação para domínios contínuos}

Em domínios contínuos, segundo o sistema cartesiano, as variáveis envolvidas serão especificadas a partir de uma origem arbitrária de referência. Neste sistema, na hipótese de forças volúmicas nulas, a aproximação do campo de deslocamentos (Eq. (2.22)), passa a ser representada por:

$$
\boldsymbol{u}_{\boldsymbol{c}}=\boldsymbol{u}_{h c}+\boldsymbol{u}_{\boldsymbol{M c}}=\sum_{j=1}^{n_{c}} \boldsymbol{c}_{j} \boldsymbol{N}_{j c}+\sum_{j=1}^{3} \overline{\boldsymbol{c}}_{j} \overline{\boldsymbol{N}}_{j c}=\boldsymbol{N}_{c} \boldsymbol{c}+\overline{\boldsymbol{N}}_{c} \overline{\boldsymbol{c}}, \text { em } \Omega
$$

onde se adotou a notação $u=u_{c}, u_{h}=u_{h c}, u_{M}=u_{M c}, \boldsymbol{N}_{j}=\boldsymbol{N}_{j c}, \overline{\boldsymbol{N}}_{j}=\overline{\boldsymbol{N}}_{j c}, \boldsymbol{N}=\boldsymbol{N}_{c}$ e $\overline{\mathbf{N}}=\overline{\mathbf{N}}_{c}$ para referir-se ao sistema cartesiano.

A seguir, descrevem-se a solução homogênea e a solução dos movimentos de corpo rígido. 


\subsubsection{Solução homogênea}

Na notação adotada, a Eq. (2.18) adquire o aspecto:

$$
L_{c}\left[D L_{c}{ }^{T}\left(u_{h c}\right)\right]=0, \text { em } \Omega
$$

sendo a solução homogênea representada por:

$$
\boldsymbol{u}_{h c}=\sum_{j=1}^{n_{c}} c_{j} \boldsymbol{N}_{j c}=\boldsymbol{N}_{c} \boldsymbol{c}, \text { em } \Omega
$$

O conjunto de vetores $\boldsymbol{N}_{j c}$, para o EPT, pode ser obtido de maneira sistemática pela formulação, em variáveis complexas, proposta por Muskhelishvili (1953). Segundo esta formulação, as componentes $u_{x}$ e $u_{y}$ do campo de deslocamentos, referentes à parcela homogênea e que satisfazem a Eq. (2.24), podem ser obtidas da equação:

$$
E\left(u_{x}+i u_{y}\right)=(3-v) \mathrm{F}(\mathrm{z})-(1+v)\left[z \overline{F^{\prime}(z)}+\overline{G(z)}\right]
$$

onde $E$ é o módulo de Young, $v$ o coeficiente de Poisson, $F(z)$ e $G(z)$ são pares de funções arbitrárias, $z=x+i y$ é um número complexo, sendo composto de uma parte real $(\operatorname{Re}(z)=x)$ e outra imaginária $(\operatorname{Im}(z)=y)$. Nesta representação $i$ é a unidade imaginária $(i=\sqrt{-1})$ e 0 conjugado de $z$, representado por $\bar{z}$,é expresso por $\bar{z}=x-i y$. A parcela $\overline{G(z)}$ é a função conjugada de $G(\bar{z})$,admitindo-se neste desenvolvimento que $z$ seja uma variável real. Já $\overline{F^{\prime}(z)}$ é a função conjugada de $F^{\prime}(\bar{z})$, sendo $F^{\prime}(z)=\frac{d F(z)}{d z}$, admitindo-se, novamente, neste desenvolvimento, que $z$ seja uma variável real. Evidentemente, após a determinação das

funções $\overline{G(z)}$ e $\overline{F^{\prime}(z)} z$ retorna com sua definição original $(z=x+i y)$. Por exemplo, se $F(z)=i z^{k} \quad$ e $\quad G(z)=i z^{k}$ ter-se-ia: $\quad F^{\prime}(z)=i k z^{k-1} \Rightarrow \overline{F^{\prime}(z)}=-i k \bar{z}^{k-1}$ e $G(\bar{z})=i \bar{z}^{k} \Rightarrow \overline{G(z)}=-i \bar{z}^{k}$, $\operatorname{com} \bar{z}=x-i y$.

Em virtude da arbitrariedade de escolha dos pares $F(z)$ e $G(z)$, uma infinidade de funções $u_{x}$ e $u_{y}$ pode ser obtida. Elas, naturalmente, podem ser interpretadas como as próprias componentes dos vetores $\boldsymbol{N}_{j c}$.

Neste trabalho, considerar-se-á o conjunto de soluções polinomiais, utilizado por Jirouseck e Venkatesh (1992), gerado a partir das seguintes escolhas para os pares de funções $F(z)$ e $G(z)$ : 


$$
\left\{\begin{array}{lll}
F(z)=i z^{k} & e & G(z)=0 \\
F(z)=z^{k} & e & G(z)=0 \\
F(z)=0 & e & G(z)=i z^{k}
\end{array} \quad ; k=1,2, \ldots, n\right.
$$

$\mathrm{Na}$ Eq. (2.27) cada par de funções gera uma sequiência de vetores linearmente independentes. A base completa de vetores $\boldsymbol{N}_{j c}$ é formada pelas componentes das seqüências oriundas dos quatro pares, denotadas por $\mathbf{N}_{\mathbf{A K}}, \mathbf{N}_{\mathbf{B K}_{\mathrm{K}}}, \mathbf{N}_{\mathrm{CK}_{\mathrm{K}}}$ e $\mathbf{N}_{\mathbf{D K}_{\mathrm{K}}}$.

Como ilustração, considere-se o par $F(z)=i z^{k}$ e $G(z)=0$, com seus desenvolvimentos $\overline{F^{\prime}(z)}=-i k \bar{z}^{k-1}$ e $\overline{G(z)}=0$. A substituição destas funções na Eq. (2.26) leva à seguinte expressão: $E\left(u_{x}+i u_{y}\right)=(3-v) i z^{\mathrm{k}}+(1+v)\left(\mathrm{ki} z^{-k-1}\right)$. Definindo-se seu termo direito como $A_{k}=(3-v) \mathrm{i} z^{\mathrm{k}}+(1+v)\left(\mathrm{ki} z \bar{z}^{\mathrm{k}-1}\right)$, por comparação, conclui-se que $E u_{x}=\operatorname{Re}\left(A_{k}\right)$ e $E u_{y}=\operatorname{Im}\left(A_{k}\right)$. Sendo $u_{x}$ e $u_{y}$ interpretadas como as componentes de $\mathbf{N}_{\mathbf{A K}}$, a seguinte seqüência de vetores é gerada:

$$
\mathbf{N}_{\mathrm{AK}}=\frac{1}{E}\left\{\begin{array}{l}
\operatorname{Re}\left(A_{k}\right) \\
\operatorname{Im}\left(A_{k}\right)
\end{array}\right\}, \text { sendo } A_{k}=(3-v) \mathrm{i} z^{\mathrm{k}}+(1+v)\left(\mathrm{ki} z \overline{\mathrm{z}}^{\mathrm{k}-1}\right) ; k=1,2, \ldots, n
$$

De maneira análoga, para os outros pares de funções, obtêm-se:

$$
\begin{aligned}
& \mathbf{N}_{\mathrm{BK}}=\frac{1}{E}\left\{\begin{array}{l}
\operatorname{Re}\left(B_{k}\right) \\
\operatorname{Im}\left(B_{k}\right)
\end{array}\right\}, \text { sendo } B_{k}=(3-v) \mathrm{z}^{\mathrm{k}}-(1+v)\left(\mathrm{k} z \overline{\mathrm{z}}^{\mathrm{k}-1}\right) ; k=1,2, \ldots, n \\
& \mathbf{N}_{\mathrm{CK}}=\frac{1}{E}\left\{\begin{array}{l}
\operatorname{Re}\left(C_{k}\right) \\
I m\left(C_{k}\right)
\end{array}\right\}, \text { sendo } C_{k}=(1+v) i \overline{\mathrm{z}}^{\mathrm{k}} ; k=1,2, \ldots, n \\
& \mathbf{N}_{\mathrm{DK}}=\frac{1}{E}\left\{\begin{array}{l}
\operatorname{Re}\left(D_{k}\right) \\
\operatorname{Im}\left(D_{k}\right)
\end{array}\right\}, \text { sendo } D_{k}=-(1+v) \overline{\mathrm{z}}^{\mathrm{k}} ; k=1,2, \ldots, n
\end{aligned}
$$

Nas Figuras $2.3,2.4,2.5$ e 2.6 estão ilustradas as partes real e imaginária de $A_{k}$ para alguns valores de $k$. Para construção destas superfícies adotou-se $v=0,2$. 


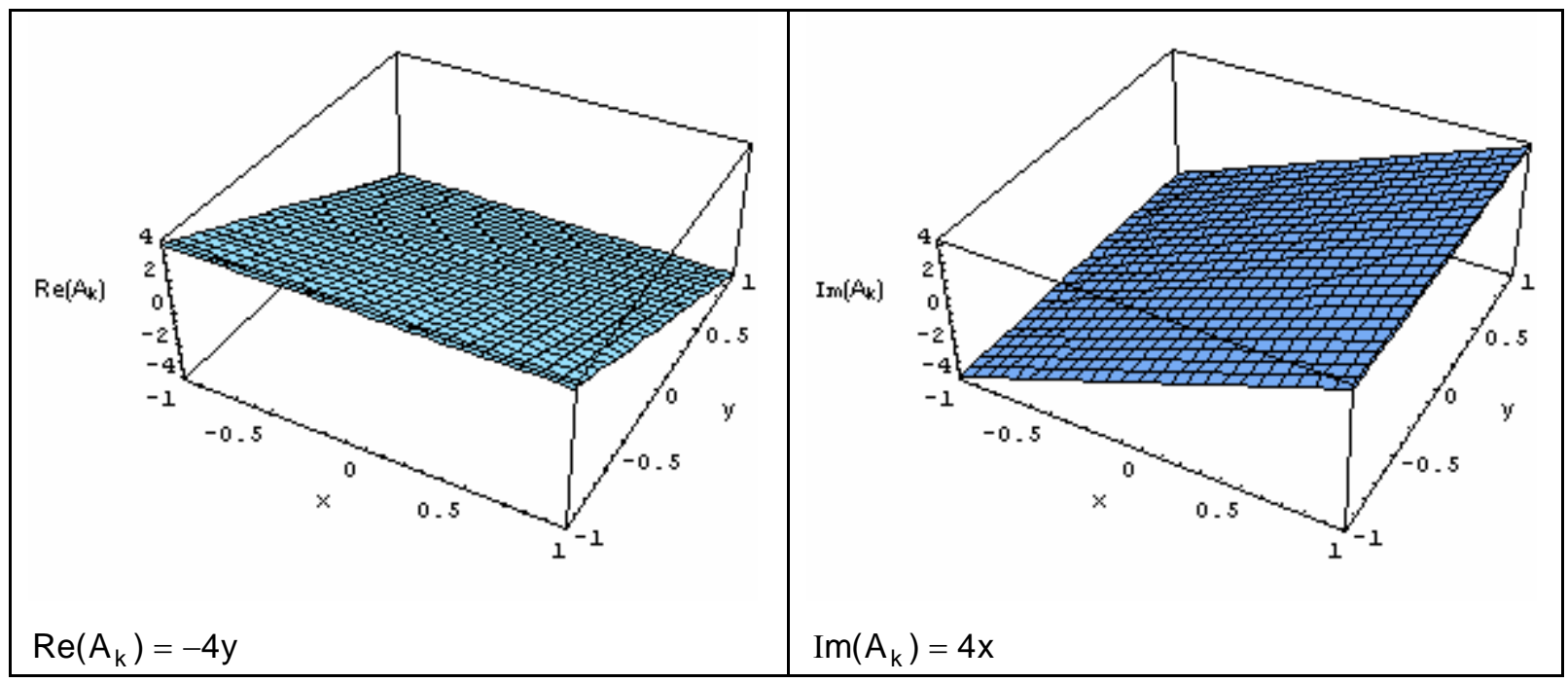

Figura 2.3. Partes real e imaginária de $A_{k}$, para $k=1$.

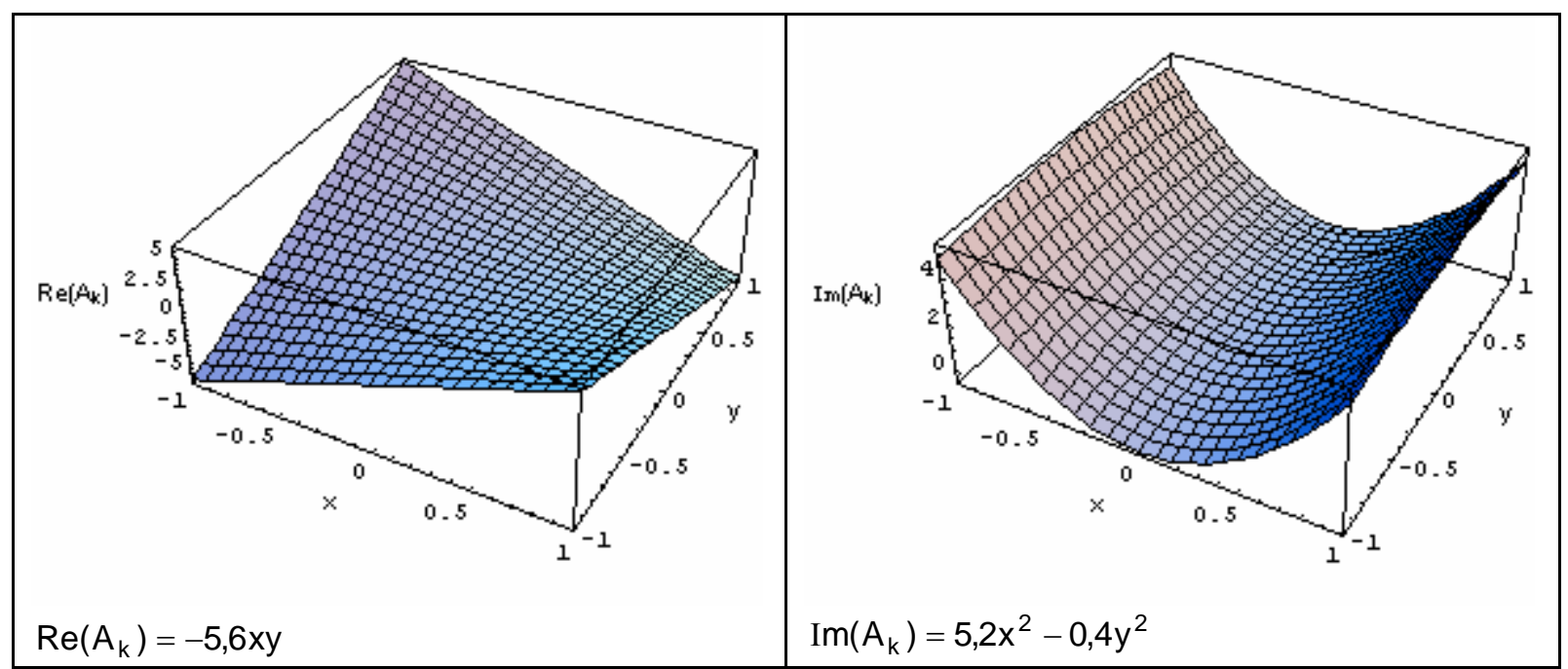

Figura 2.4. Partes real e imaginária de $A_{k}$, para $k=2$.

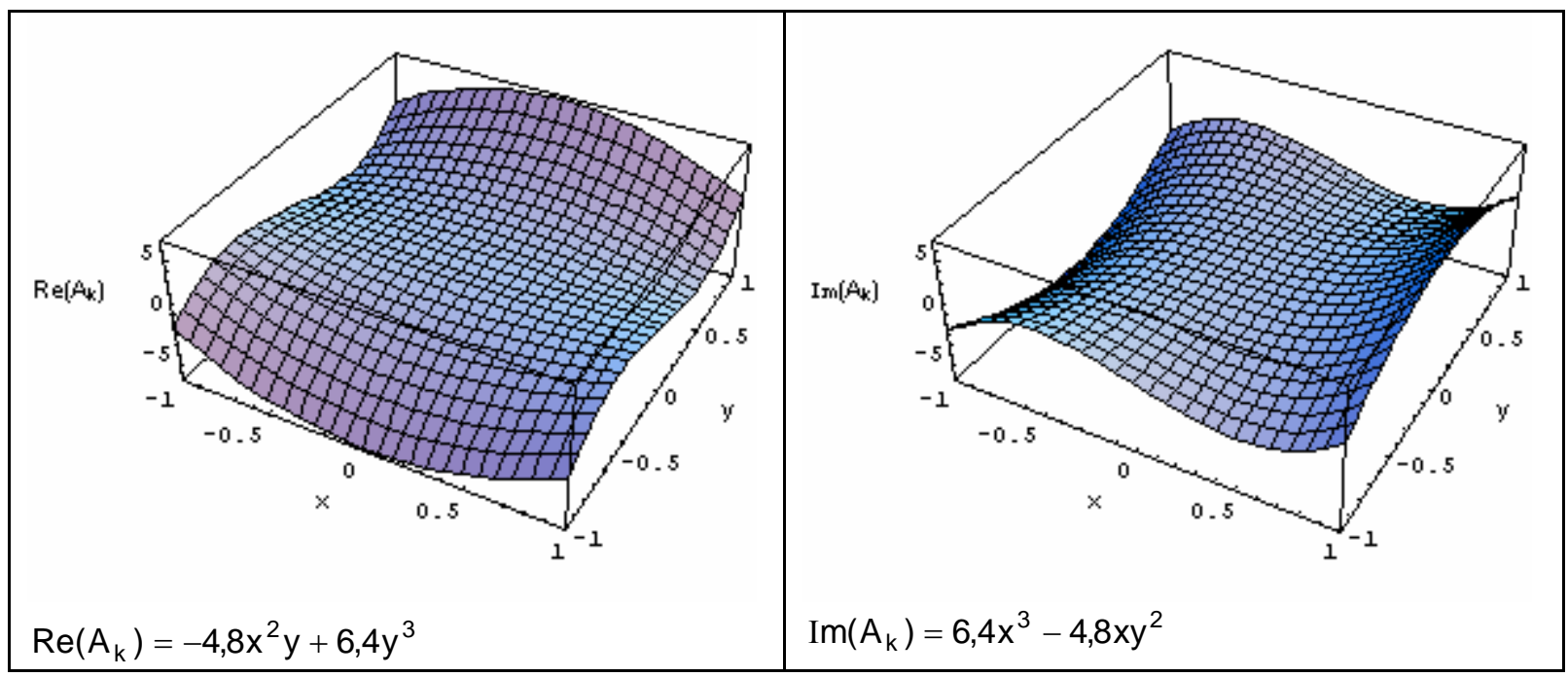

Figura 2.5. Partes real e imaginária de $A_{k}$, para $k=3$. 


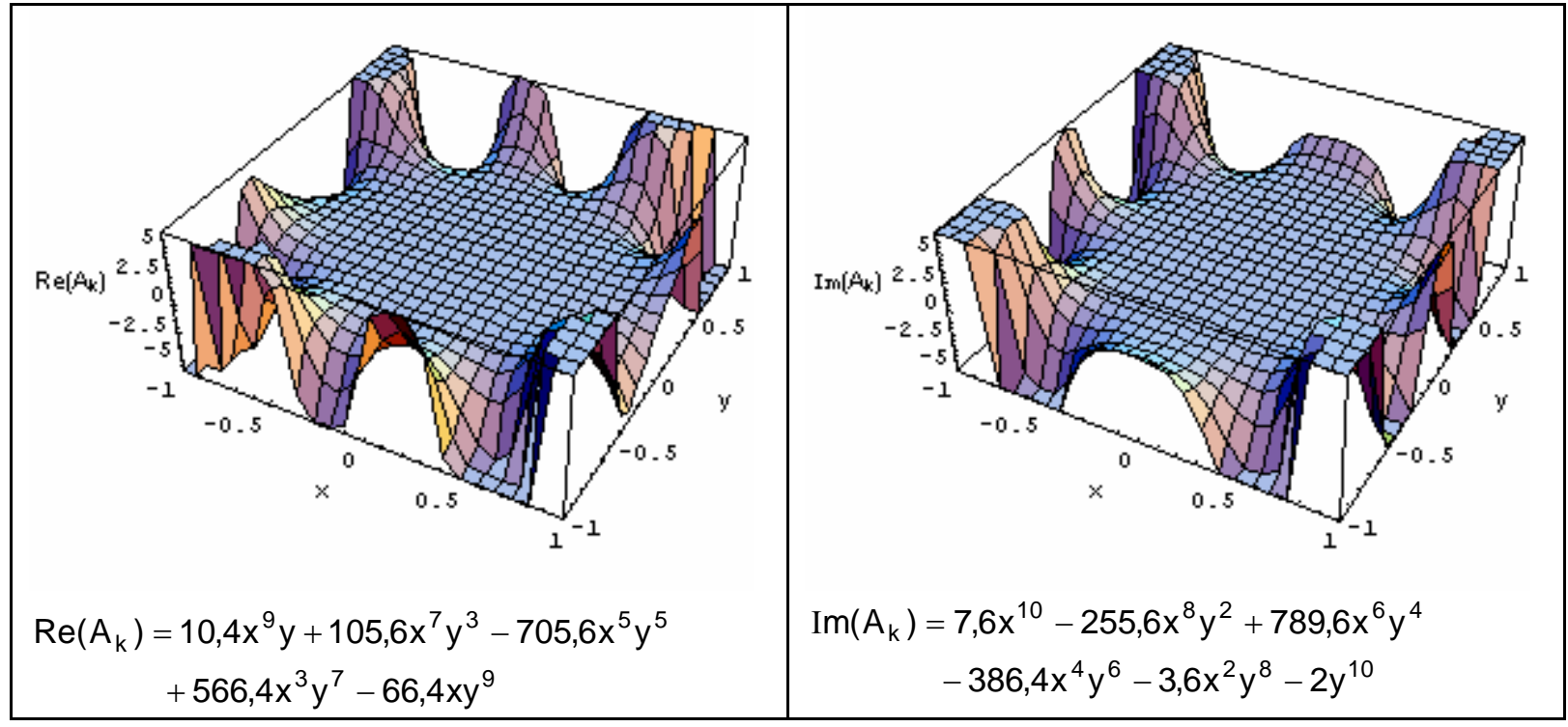

Figura 2.6. Partes real e imaginária de $A_{k}$, para $k=10$.

Iniciando-se a sequiência para $k=1$, observa-se que os deslocamentos obtidos pela Eq. (2.28) representam movimentos de rotação de corpo rígido. De fato, para $k=1$ os deslocamentos são expressos por $u_{x}=-4 E^{-1} y$ e $u_{y}=4 E^{-1} x ;$ assim, a rotação $\omega=\frac{1}{2}\left(\frac{\partial u_{x}}{\partial y}-\frac{\partial u_{y}}{\partial x}\right)=-4 E^{-1}$ é uma constante não nula. Portanto, na construção da seqüência dos vetores $\boldsymbol{N}_{j c}$, deve-se descartar a contribuição de $\boldsymbol{N}_{\boldsymbol{A} 1}$, que, aliás, é um dos vetores que compõe a matriz $\overline{\boldsymbol{N}}_{c}$.

Diante da observação anterior, conclui-se que o número $n_{c}$ de vetores $\boldsymbol{N}_{j c}$, ou de coeficientes $c_{j}$ da Eq. (2.25), obtido a partir das seqüências de vetores $\boldsymbol{N}_{A k}, \boldsymbol{N}_{B k}, \boldsymbol{N}_{C k}$ e $\boldsymbol{N}_{D k}$, com $k=1, \cdots, n$ é igual a $n_{c}=4 n-1$. Finalmente, a matriz $\boldsymbol{N}_{c}$ adquire o aspecto:

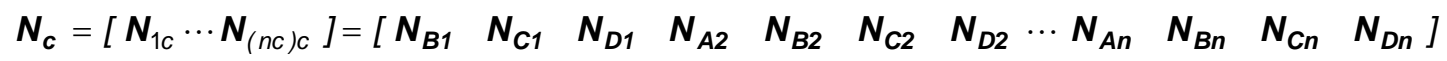

De acordo com as Eqs. (2.28)-(2.31), $\boldsymbol{N}_{c}$ pode ser representada por:

$$
\begin{array}{r}
\boldsymbol{N}_{c}=\frac{1}{E}\left[\begin{array}{llllll}
\operatorname{Re}\left(B_{1}\right) & \operatorname{Re}\left(C_{1}\right) & \operatorname{Re}\left(D_{1}\right) & \operatorname{Re}\left(A_{2}\right) & \operatorname{Re}\left(B_{2}\right) & \operatorname{Re}\left(C_{2}\right) \\
\operatorname{Im}\left(B_{1}\right) & \operatorname{Im}\left(C_{1}\right) & \operatorname{Im}\left(D_{1}\right) & \operatorname{Im}\left(A_{2}\right) & \operatorname{Im}\left(B_{2}\right) & \operatorname{Im}\left(C_{2}\right) \\
& \\
\operatorname{Re}\left(D_{2}\right) & \ldots & \operatorname{Re}\left(A_{n}\right) & \operatorname{Re}\left(B_{n}\right) & \operatorname{Re}\left(C_{n}\right) & \operatorname{Re}\left(D_{n}\right) \\
\operatorname{Im}\left(D_{2}\right) & \cdots & \operatorname{Im}\left(A_{n}\right) & \operatorname{Im}\left(B_{n}\right) & \operatorname{Im}\left(C_{n}\right) & \operatorname{Im}\left(D_{n}\right)
\end{array}\right]
\end{array}
$$

No apêndice (A), encontra-se a expansão de $\boldsymbol{N}_{c}$ até a $47^{\mathrm{a}}$ coluna. 


\subsubsection{Solução dos movimentos de corpo rígido}

A solução para os movimentos de corpo rígido, obtida pela mesma restrição imposta à solução homogênea, é da forma:

$$
\boldsymbol{u}_{M c}=\sum_{j=1}^{3} \bar{c}_{j} \overline{\boldsymbol{N}}_{j c}=\overline{\boldsymbol{N}}_{c} \bar{c}, \text { em } \Omega
$$

sendo $\overline{\boldsymbol{N}}_{c}=\left[\begin{array}{ccc}1 & 0 & -4 y / E \\ 0 & 1 & 4 x / E\end{array}\right]$.

Observa-se que as duas primeiras colunas da matriz $\overline{\boldsymbol{N}}_{c}$ representam os movimentos de translação enquanto a terceira decorre da rotação, que é justamente o vetor $\boldsymbol{N}_{\boldsymbol{A 1}}$.

\subsubsection{Aproximação para domínios com orifício circular}

Quando da existência de um orifício circular, para obtenção das soluções em deslocamento: homogênea e de corpo rígido, considera-se o domínio $\Omega$ e o sistema de coordenadas polares com origem no centro do orifício, conforme indicado na Figura 2.7.

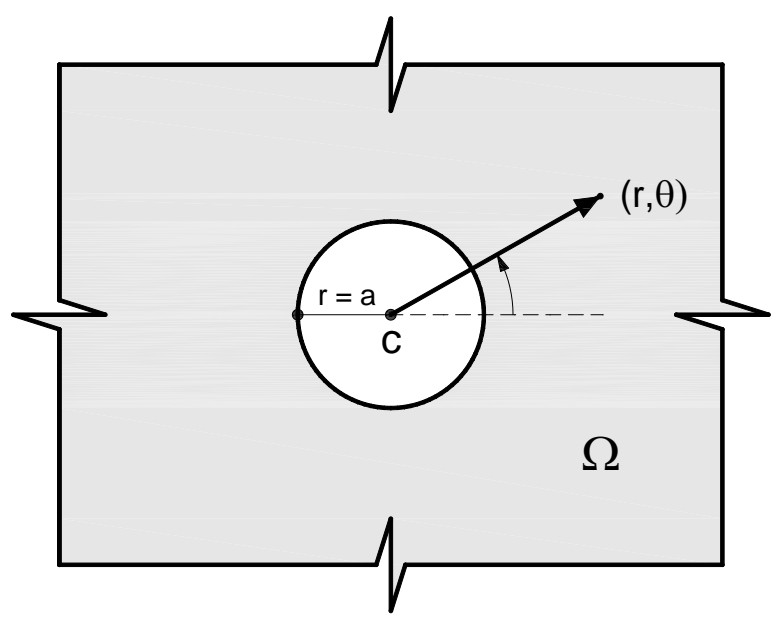

Figura 2.7. Domínio com orifício circular.

Em problemas com este tipo de domínio, a adoção do sistema de coordenadas polares permite que condições de contorno em forças sejam aplicadas diretamente ao longo da circunferência. Esta estratégia é fundamental na construção da solução homogênea, como será descrito adiante.

Neste sistema de coordenadas, na hipótese de forças volúmicas nulas, a aproximação 
do campo de deslocamentos (Eq. (2.22)) passa a ser representada por:

$$
\boldsymbol{u}_{\boldsymbol{p}}=\boldsymbol{u}_{h p}+\boldsymbol{u}_{\boldsymbol{M p}}=\sum_{j=1}^{n_{C}} c_{j} \boldsymbol{N}_{j p}+\sum_{j=1}^{3} \bar{c}_{j} \overline{\boldsymbol{N}}_{\boldsymbol{j p}}=\boldsymbol{N}_{p} \boldsymbol{c}+\overline{\boldsymbol{N}}_{\boldsymbol{p}} \overline{\boldsymbol{c}}, \text { em } \Omega
$$

onde se adotou a notação $\boldsymbol{u}=\boldsymbol{u}_{p}, \boldsymbol{u}_{\boldsymbol{h}}=\boldsymbol{u}_{\boldsymbol{h} p}, \boldsymbol{u}_{M}=\boldsymbol{u}_{\boldsymbol{M p}}, \boldsymbol{N}_{\boldsymbol{j}}=\boldsymbol{N}_{j p}, \overline{\boldsymbol{N}}_{\boldsymbol{j}}=\overline{\boldsymbol{N}}_{j p}, \boldsymbol{N}=\boldsymbol{N}_{\boldsymbol{p}}$ e $\overline{\boldsymbol{N}}=\overline{\boldsymbol{N}}_{\boldsymbol{p}}$, para referir-se ao sistema de coordenadas polares.

\subsubsection{Solução homogênea}

Na notação adotada, a Eq. (2.18) adquire o aspecto:

$$
M_{\sigma} D M_{\varepsilon} u_{h p}+M_{\sigma} D L_{p}^{T}\left(u_{h p}\right)+L_{p}\left(D M_{\varepsilon} u_{h p}\right)+L_{p}\left[D L_{p}^{T}\left(u_{h p}\right)\right]=0, \text { em } \Omega
$$

sendo a solução homogênea representada por:

$$
\boldsymbol{u}_{h p}=\sum_{j=1}^{n_{p}} c_{j} \boldsymbol{N}_{j p}=\boldsymbol{N}_{p} c, \text { em } \Omega
$$

O conjunto de vetores $\boldsymbol{N}_{j p}$, para EPT, pode ser obtido a partir da Eq. (2.35), admitindo-se soluções da forma:

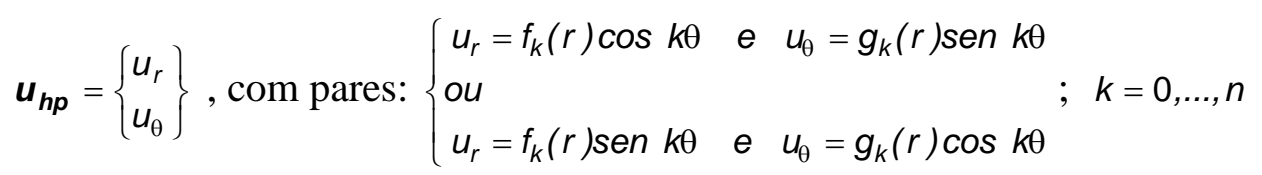

onde $f_{k}(r)$ e $g_{k}(r)$ são funções de $r$.

Cada par de soluções da Eq. (2.37) quando substituído na Eq. (2.35) conduz a um sistema de equações diferenciais ordinárias (Euler-Cauchy) em $f_{k}(r)$ e $g_{k}(r)$. Para o primeiro par, onde $u_{r}=f_{k}(r) \cos k \theta$ e $u_{\theta}=g_{k}(r) \operatorname{sen} k \theta$, a resolução do sistema leva às seguintes expressões para as funções $f_{k}$ e $g_{k}$ :

$$
\begin{aligned}
& \left\{\begin{array}{l}
f_{0}(r)=C_{01} r+C_{02} r^{-1} \\
g_{0}(r)=\frac{2}{1-v} C_{01} r+C_{02} r^{-1}
\end{array} ; \text { para } k=0\right. \\
& \left\{\begin{array}{l}
f_{1}(r)=C_{11} r^{2}+C_{12} r^{-2}+C_{13}+C_{14} \ln r \\
g_{1}(r)=\frac{5+v}{1-3 v} C_{11} r^{2}+C_{12} r^{-2}-C_{13}-C_{14} \ln r
\end{array} ; \text { para } k=1\right. \\
& \left\{\begin{array}{l}
f_{2}(r)=-4 v C_{21} r^{3}+C_{22} r^{-3}+C_{23} r^{-1}+C_{24} r \\
g_{2}(r)=2(3+v) C_{21} r^{3}+C_{22} r^{-3}-\frac{1-v}{2} C_{23} r^{-1}-C_{24} r
\end{array} \text {; para } k=2\right.
\end{aligned}
$$




$$
\left\{\begin{array}{l}
f_{k}(r)=C_{k 1} r^{1+k}+C_{k 2} r^{-1-k}+C_{k 3} r^{1-k}+C_{k 4} r^{-1+k} \\
g_{k}(r)=\lambda_{k} C_{k 1} r^{1+k}+C_{k 2} r^{-1-k}+\bar{\lambda}_{k} C_{k 3} r^{1-k}-C_{k 4} r^{-1+k}
\end{array} \quad ; \text { para } k \geq 3\right.
$$

sendo $\lambda_{k}=\frac{4+k(1+v)}{2(1-v)-k(1+v)}$ e $\bar{\lambda}_{k}=\frac{-4+k(1+v)}{2(1-v)+k(1+v)}$.

As expressões encontradas para as funções $f_{k}$ e $g_{k}$ são absolutamente gerais, sem vínculo algum com as dimensões do orifício ou com as forças de superfície ali atuantes. Por conseguinte, para que o par $u_{r}=f_{k}(r) \cos k \theta$ e $u_{\theta}=g_{k}(r) \operatorname{sen} k \theta$ constitua a solução procurada, as condições de contorno em forças ao longo da circunferência devem ser impostas, quais sejam:

$$
\left\{\begin{array}{l}
\sigma_{r}=0 \\
\tau_{r \theta}=0
\end{array} \quad ; e m r=a\right.
$$

A rigor, numa análise mais geral, as condições de contorno deveriam ser aplicadas ao campo de tensões obtido a partir da aproximação do campo de deslocamentos ditado pela Eq. (2.16), isto é, ao campo de tensões do problema, construído a partir das soluções em deslocamentos: particular, homogênea e de corpo rígido. Contudo, em virtude da premissa adotada de forças volúmicas nulas e das características da solução de corpo rígido, infere-se que elas podem ser aplicadas diretamente ao campo de tensões obtido apenas a partir da solução homogênea. Neste contexto, tal campo passa a representar as tensões resultantes do problema, uma vez que estão associadas tensões nulas tanto à solução particular, quanto à solução de corpo rígido.

Assim sendo, mediante aplicação das condições de contorno, os vetores $\boldsymbol{N}_{j p}$ da solução homogênea podem ser determinados sem maiores dificuldades. Como ilustração, considere-se a solução para $k=0$. Neste caso, de acordo com as Eqs. (2.37) e (2.38), tem-se a primeira parcela da solução homogênea:

$$
u_{1 p}=\left\{\begin{array}{l}
u_{r} \\
u_{\theta}
\end{array}\right\}=\left\{\begin{array}{l}
C_{01} r+C_{02} r^{-1} \\
0
\end{array}\right\}
$$

A partir da equação de compatibilidade (Eq. (2.4)) e da relação constitutiva (Eq. (2.6)), obtém-se o tensor de tensões:

$$
\sigma_{1 p}=\left\{\begin{array}{l}
\sigma_{r} \\
\sigma_{\theta} \\
\tau_{r \theta}
\end{array}\right\}=\frac{1}{-r^{2}+r^{2} v^{2}}\left\{\begin{array}{l}
C_{02}(1-v)-C_{01} r^{2}(1+v) \\
C_{02}(-1+v)-C_{01} r^{2}(1+v) \\
0
\end{array}\right\}
$$

Impondo-se as condições de contorno, obtém-se a relação entre as constantes $\mathrm{C}_{01} \mathrm{e}$ $\mathrm{C}_{02}$ : 


$$
C_{02}=\frac{a^{2}(1+v)}{1-v} C_{01}
$$

Finalmente a partir das Eqs. (2.43) e (2.45), obtém-se o primeiro termo da solução procurada, onde se identifica diretamente o grau de liberdade $c_{1}$ e o vetor $\boldsymbol{N}_{1 p}$ :

$$
u_{1 p}=\underbrace{C_{01}}_{c 1} \underbrace{\left\{\begin{array}{c}
\frac{-r^{2}(1-v)+a^{2}(1+v)}{r-N} \\
0
\end{array}\right\}}_{\mathbf{N}_{1 \mathrm{p}}}
$$

Repetindo-se este procedimento, para $k=1, k=2$ e $k \geq 3$, obtêm-se os demais termos da solução homogênea referentes ao primeiro par de soluções da Eq. (2.37). Para o outro par, devem-se determinar as novas expressões das funções $f_{k}$ e $g_{k}$ e proceder de maneira análoga. Contudo, observa-se que nem todos os vetores obtidos neste processo compõem a matriz $\boldsymbol{N}_{\boldsymbol{p}}$, já que três deles contêm movimentos de corpo rígido. É o que ocorre para o segundo par quando $k=0$ e para ambos pares quando $k=1$. Estes vetores, naturalmente, compõem a matriz $\bar{N}_{p}$.

No apêndice (B), encontra-se a lei de formação da Matriz $\boldsymbol{N}_{\boldsymbol{p}}$.

\subsubsection{Solução dos movimentos de corpo rígido}

A solução para os movimentos de corpo rígido, obtida pela mesma restrição imposta à solução homogênea, é da forma:

$$
\boldsymbol{u}_{\mathbf{M p}}=\sum_{j=1}^{3} \overline{\boldsymbol{c}}_{j} \overline{\boldsymbol{N}}_{j p}=\overline{\boldsymbol{N}}_{p} \overline{\boldsymbol{c}}, \text { em } \Omega
$$

sendo $\overline{\boldsymbol{N}}_{p}=\left[\begin{array}{ccc}\cos \theta & \operatorname{sen} \theta & 0 \\ -\operatorname{sen} \theta & \cos \theta & r\end{array}\right]$.

Observa-se que as duas primeiras colunas da matriz $\overline{\boldsymbol{N}}_{c}$ representam os movimentos de translação enquanto a terceira decorre da rotação. 


\subsection{Aproximação do campo de tensões no domínio}

A aproximação do campo de tensões pode ser obtida a partir da aproximação do campo de deslocamentos, mediante aplicação da equação de compatibilidade e da relação constitutiva.

Assim sendo, na hipótese de forças volúmicas nulas, utilizando-se as Eqs. (2.23), (2.3) e (2.5), a aproximação do campo de tensões para domínios contínuos pode ser expressa por:

$$
\boldsymbol{\sigma}_{c}=\sum_{j=1}^{n c} c_{j} \boldsymbol{S}_{j c}=\boldsymbol{S}_{c} \mathbf{c}, \text { em } \Omega
$$

sendo $\boldsymbol{S}_{j c}=D L_{c}{ }^{T} \boldsymbol{N}_{j c}$ e $\boldsymbol{S}_{c}=D L_{c}{ }^{T} \boldsymbol{N}_{c}$.

No apêndice (A), encontra-se a expansão de $\boldsymbol{S}_{c}$ até a $47^{\mathrm{a}}$ coluna.

De maneira análoga, a aproximação de tensões para domínios com orifício circular pode ser obtida utilizando-se as Eqs. (2.34), (2.4) e (2.6):

$$
\boldsymbol{\sigma}_{p}=\sum_{j=1}^{n c} c_{j} \boldsymbol{S}_{j p}=\boldsymbol{S}_{p} \boldsymbol{c}, \mathrm{em} \Omega
$$

sendo $S_{j p}=D M_{\varepsilon} N_{j p}+D L_{p}^{T} N_{j p}$ e $S_{p}=D M_{\varepsilon} N_{p}+D L_{p}^{T} N_{p}$.

A lei de formação da matriz $\boldsymbol{S}_{\boldsymbol{p}}$ encontra-se no apêndice (B).

\subsection{Aproximação do campo de tensões no contorno}

A aproximação do campo de tensões no contorno pode ser obtida da aproximação do campo de tensões no domínio, aplicando-se o teorema de Cauchy.

Para domínios contínuos, onde as variáveis estão especificadas para o sistema de coordenadas cartesianas, a aplicação do teorema é direta. Assim, na hipótese de forças volúmicas nulas, das Eqs. (2.10) e (2.48), tem-se:

$$
\boldsymbol{t}_{\mathrm{c}}=\boldsymbol{A} \boldsymbol{\sigma}_{c}=\sum_{j=1}^{n c} c_{j} \boldsymbol{T}_{j c}=\boldsymbol{T}_{c} \mathbf{c}, \mathrm{em} \Gamma
$$

sendo $\boldsymbol{T}_{j c}=\boldsymbol{A} \boldsymbol{S}_{j c}$ e $\boldsymbol{T}_{c}=\boldsymbol{A} \boldsymbol{S}_{c}$.

Por outro lado, em domínios com orifício circular, onde as variáveis estão especificadas para o sistema de coordenadas polares, deve-se primeiro transferir a aproximação de tensões no domínio (Eq. (2.49)) para o sistema cartesiano mediante a Eq. 
(2.8) e em seguida aplicar o teorema (Eq. (2.10)). Por conseguinte, a aproximação de tensões no contorno também é especificada para o sistema cartesiano e, na hipótese de forças volúmicas nulas, adquire o aspecto:

$$
\boldsymbol{t}_{p}=\boldsymbol{A}\left(\boldsymbol{R}_{\boldsymbol{\sigma \theta}} \boldsymbol{\sigma}_{\boldsymbol{p}}\right)=\sum_{j=1}^{n c} c_{j} \boldsymbol{T}_{j p}=\boldsymbol{T}_{p} \mathbf{c}, \text { em } \Gamma
$$

sendo agora $\boldsymbol{T}_{j p}=\boldsymbol{A} \boldsymbol{R}_{\sigma \theta} \boldsymbol{S}_{j p}$ e $\boldsymbol{T}_{p}=\boldsymbol{A} \boldsymbol{R}_{\sigma \theta} \boldsymbol{S}_{p}$. 


\section{Formulação híbrida-Trefftz em problemas bidimensionais da Elasticidade}

\subsection{Considerações iniciais}

No capítulo anterior apresentaram-se os fundamentos para a concepção das funções aproximativas utilizadas em problemas bidimensionais da Elasticidade baseadas no Método de Trefftz, as quais, teoricamente, poderiam ser utilizadas em qualquer método numérico. Em particular, neste capítulo, apresenta-se uma formulação em elementos finitos, designada por formulação híbrida-Trefftz, que utiliza as referidas funções na aproximação do campo de tensões no domínio do elemento e funções auxiliares na aproximação do campo de deslocamentos no contorno do elemento.

Tal formulação consagrou dois modelos de elementos da literatura, o HTD ("hybrid Trefftz displacement") que pode ser encontrado, por exemplo, no trabalho de Jirouseck e Wróblewski (1996) e o HTS ("hybrid Trefftz stress") apresentado por Freitas (1998). Na Figura 3.1 encontram-se suas representações no caso bidimensional.

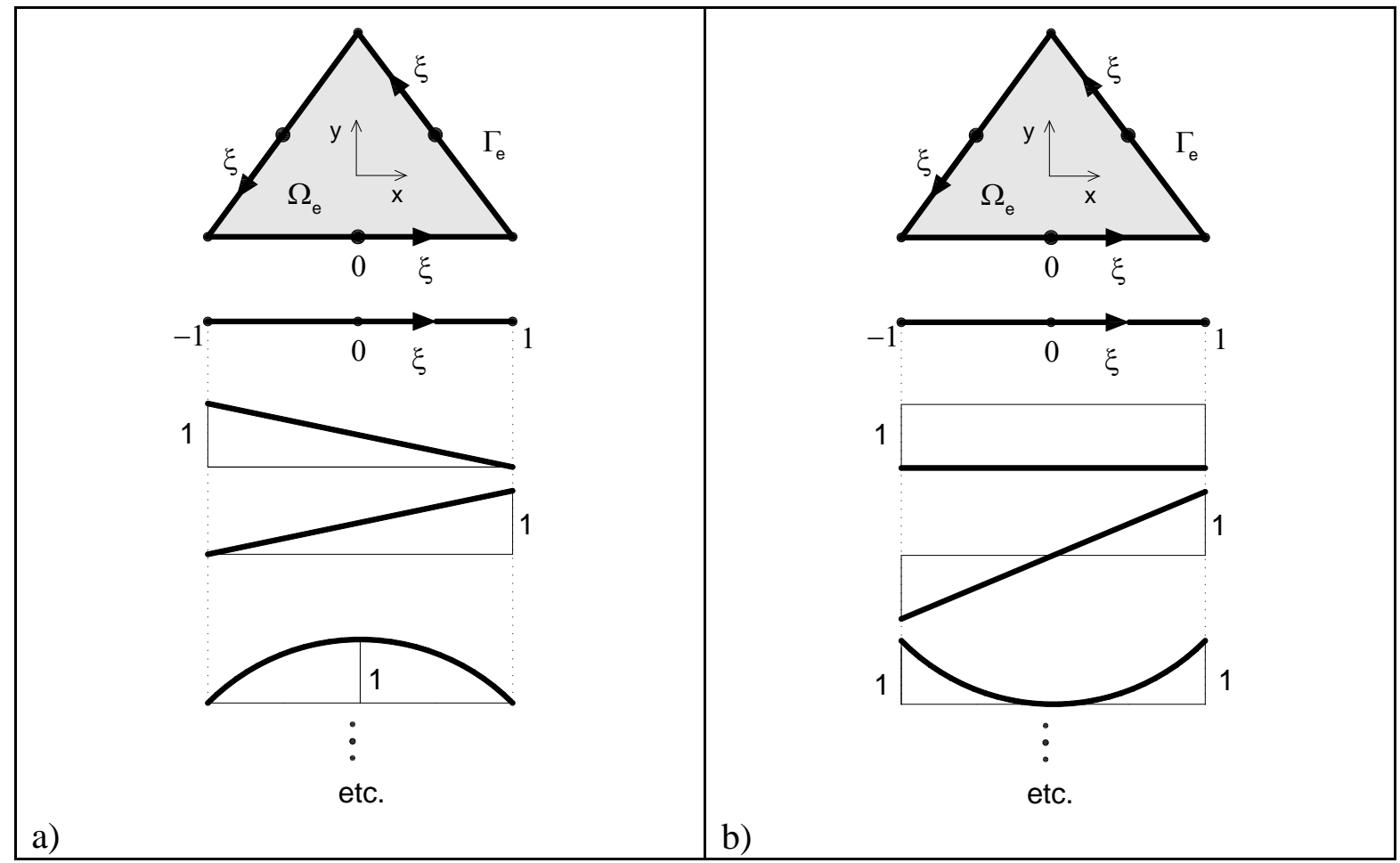

Figura 3.1. (a) HTD: campo aproximado de deslocamentos contínuo em $\Gamma_{\mathrm{e}}$ e (b) HTS: campo aproximado de deslocamentos em $\Gamma_{\mathrm{e}}$ descontínuo nas quinas. 
Apesar da nomenclatura utilizada, ambos os modelos, aproximam diretamente tensões no domínio $\Omega_{\mathrm{e}}$ e deslocamentos no contorno $\Gamma_{\mathrm{e}}$, tratando-se em essência de formulações de tensão. A aproximação de deslocamentos em $\Omega_{\mathrm{e}}$ pode ser obtida indiretamente, a partir dos valores nodais de deslocamentos aproximados diretamente em $\Gamma_{e}$. Neste sentido um outro método numérico, deve ser utilizado no pós-processamento, como, por exemplo, o Método dos Mínimos Quadrados. Entretanto, infere-se que na maioria das análises este procedimento é supérfluo, uma vez que sempre é possível aproximar diretamente o deslocamento de qualquer ponto mediante uma discretização conveniente. Talvez isto explique por que tal procedimento, característico do HTD, tenha sido negligenciado na formulação do HTS.

A diferença fundamental entre estes modelos caracteriza-se pela imposição ou não da continuidade na aproximação do campo de deslocamentos no contorno do elemento. No HTD impõe-se a continuidade do campo de deslocamentos em todo contorno $\Gamma_{\mathrm{e}}$ mediante a compatibilidade dos graus de liberdade nodais, enquanto, no HTS a aproximação é construída para cada lado de forma independente, resultando numa descontinuidade em cada quina de $\Gamma_{\mathrm{e}}$. Segundo Freitas (1998) tal descontinuidade, ocasionava maior continuidade na aproximação de tensões, característica essencial na análise de problemas singulares.

Neste trabalho, apesar da constatação de Freitas, referente à aproximação de tensões, opta-se pela continuidade na aproximação do campo de deslocamentos em $\Gamma_{\mathrm{e}}$. Tal opção não é meramente fortuita, uma vez que se utilizarão, conforme serão apresentadas no capítulo seguinte, técnicas de enriquecimento que promovem melhorias em todos os campos aproximados, inclusive no que concerne à continuidade da aproximação de tensões.

Por mera questão de organização do trabalho, apresenta-se neste capítulo a formulação híbrida-Trefftz, aplicada a problemas bidimensionais da Elasticidade, de forma simplificada, particularmente no tocante à aproximação do campo de deslocamentos no contorno do elemento, a qual é construída a partir de funções de forma lineares. Já no capítulo seguinte ela será estendida na obtenção de uma formulação mais geral.

Assim sendo, primeiramente, apresentam-se o elemento finito com domínio contínuo utilizado e as características dos campos aproximados. Logo em seguida, descreve-se o equacionamento da referida formulação, no tocante tanto aos princípios variacionais utilizados quanto às equações de compatibilidade, dos deslocamentos e das tensões, impostas na montagem do sistema global. Por fim, descrevem-se as particularidades do elemento finito com orifício circular. 


\subsection{Elemento finito híbrido-Trefftz em domínios contínuos}

Adota-se, neste trabalho, o elemento finito híbrido-Trefftz quadrilateral de quatro nós. Para aproximação de tensões no domínio do elemento, utilizam-se coordenadas cartesianas e estabelece-se como origem o centróide. Já para a aproximação do campo de deslocamentos no contorno, em virtude das funções de forma, especifica-se, para cada lado, um sistema local de coordenadas adimensionais com origem no respectivo ponto médio, conforme indicado na Figura 3.2.

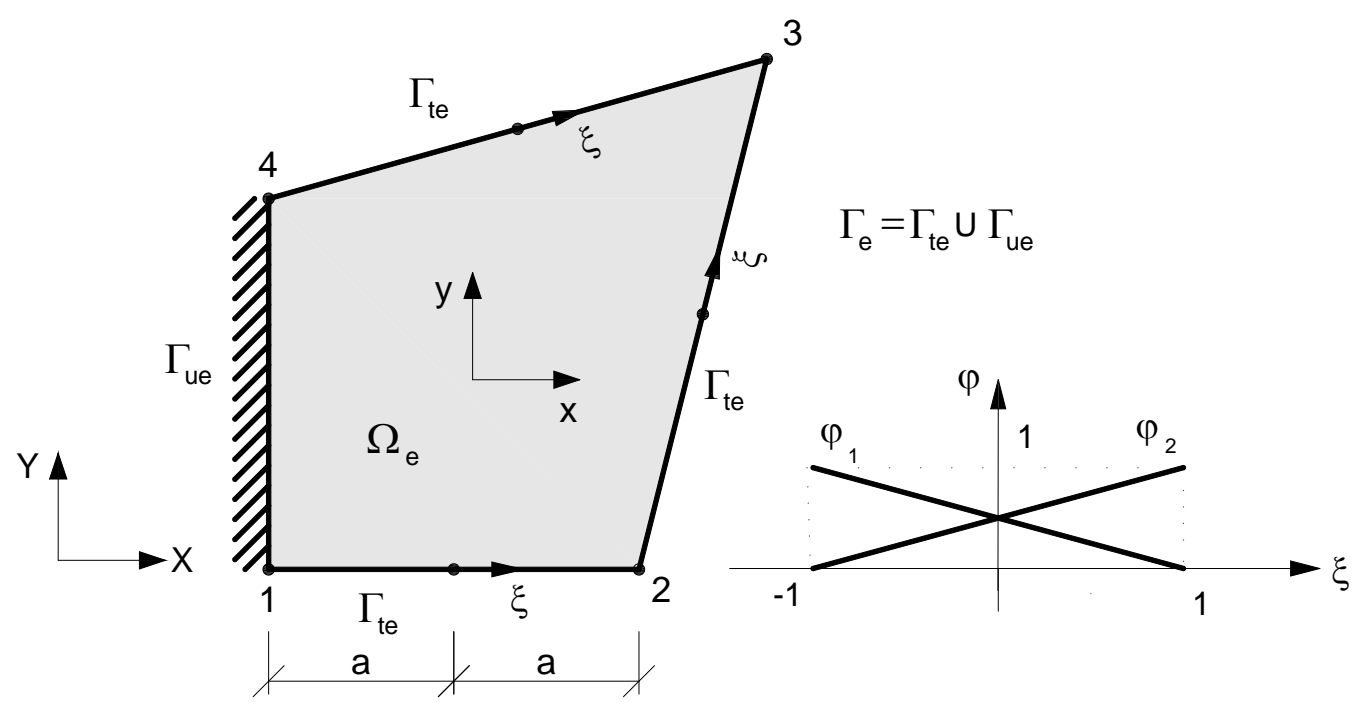

Figura 3.2. Elemento finito híbrido-Trefftz quadrilateral de quatro nós e funções de forma associadas a um lado arbitrário.

\subsubsection{Aproximação do campo de tensões}

\subsubsection{Aproximação no domínio do elemento}

A aproximação do campo de tensões dada pela Eq. (2.48), tomada para um número conveniente de termos e particularizada para o domínio do elemento adquire, doravante, a forma:

$$
\boldsymbol{\sigma}_{\boldsymbol{c}}=\boldsymbol{S}_{\boldsymbol{c}} \mathbf{c}, \mathrm{em} \Omega_{\mathrm{e}}
$$

sendo $S_{c}=D L_{c}{ }^{T} N_{c}$. 
O campo aproximado de tensões $\sigma_{c}$ é auto-equilibrado, uma vez que satisfaz, a menos das forças volúmicas, a equação de equilíbrio. Isto pode ser verificado, partindo-se da premissa que $\sigma_{c}$ é derivado da solução da equação de Navier, mediante a equação de compatibilidade e da relação constitutiva Assim, a partir das Eqs. (2.5), (2.3) e (2.12), obtémse:

$$
L_{c}\left[\sigma_{c}\right]=L_{c}\left[D \varepsilon_{c}\right]=L_{c}\left[D L_{c}{ }^{T}\left(u_{c}\right)\right]=\Re\left(u_{c}\right)=0, \text { em } \Omega_{\mathrm{e}}
$$

Como a aproximação dada pela Eq. (3.1) deve ser válida independentemente do vetor $\boldsymbol{c}$, também é imediato que:

$$
\boldsymbol{L}_{c} \boldsymbol{S}_{c}=\mathbf{0}, \text { em } \Omega_{\mathrm{e}}
$$

Além de satisfazer o equilíbrio local, a construção da aproximação mediante campos auto-equilibrados de tensões, como será visto adiante no equacionamento da formulação híbrida-Trefftz, permite que as integrações no domínio $\Omega_{\mathrm{e}}$ sejam realizadas apenas no contorno $\Gamma_{\mathrm{e}}$. Este fato facilita todo o processo de implementação, principalmente em modelos de elementos que apresentem geometria irregular.

\subsubsection{Aproximação no contorno do elemento}

A aproximação de tensões no contorno pode ser obtida da aproximação de tensões no domínio, aplicando-se o teorema de Cauchy. Assim, das Eqs. (3.1) e (2.10), tem-se:

$$
\boldsymbol{t}_{\boldsymbol{c}}=\boldsymbol{T}_{\boldsymbol{c}} \mathbf{c}, \mathrm{em} \Gamma_{\mathrm{e}}
$$

sendo $\boldsymbol{T}_{c}=\boldsymbol{A} \boldsymbol{S}_{c}$.

\subsubsection{Aproximação do campo de deslocamentos no contorno}

Sendo os deslocamentos conhecidos e impostos em forma forte na fronteira cinemática $\Gamma_{\text {ue }}$, a aproximação do campo de deslocamentos no contorno passa a ser restrita à fronteira estática $\Gamma_{\text {te }}$. Ela pode ser obtida mediante combinação linear de funções de forma associadas aos lados do elemento (vide Figura 3.2). Assim sendo, tem-se:

$$
\tilde{\mathbf{u}}=\tilde{\mathbf{U}} \mathbf{d} ; \mathrm{em} \Gamma_{\text {te }}
$$


onde $\tilde{u}$ é aproximação do campo de deslocamentos, $\tilde{\boldsymbol{U}}$ a matriz que coleta as funções de forma e $\mathbf{d}$ o vetor composto pelos graus de liberdade em deslocamentos.

Evidentemente, em decorrência da discretização adotada para o contorno, a aproximação deve ser realizada por trechos. Por exemplo, na Figura 3.2, considerando-se o lado que contêm os pontos 1 e 2 , as funções de forma escritas em função de coordenadas adimensionais $\xi=s / a$ com $s \in[-a$, a $]$, são expressas por:

$$
\left\{\begin{array}{l}
\text { - } \varphi_{1}=\frac{1}{2}(-\xi+1) \\
\text { - } \varphi_{2}=\frac{1}{2}(+\xi+1)
\end{array}\right.
$$

Para este lado, considerando-se os graus de liberdade do nó $1\left\{u_{x 1}, u_{y 1}\right\}$ e os do nó 2 $\left\{u_{x 2}, u_{y 2}\right\}$, o campo de deslocamentos aproximado adquire a forma:

$$
\tilde{\mathbf{u}}_{12}=\tilde{\boldsymbol{U}}_{\mathbf{1 2}} \boldsymbol{d}_{12}
$$

onde:

- $\tilde{u}_{12}{ }^{T}=\left\{\begin{array}{ll}\tilde{u}_{x 12} & \tilde{u}_{y 12}\end{array}\right\}$ é a aproximação do campo de deslocamentos expresso por suas componentes.

- $\tilde{\boldsymbol{U}}_{12}=\left[\begin{array}{ll}\tilde{\boldsymbol{U}}_{\varphi_{1}} & \tilde{\boldsymbol{U}}_{\varphi_{2}}\end{array}\right]=\left[\begin{array}{ll}\varphi_{1} \boldsymbol{I}_{2} & \varphi_{2} \boldsymbol{I}_{2}\end{array}\right]$ é a matriz que coleta as funções de forma do lado considerado, sendo $I_{2}$ a matriz identidade de ordem 2 .

- $\boldsymbol{d}_{12}{ }^{T}=\left\{\begin{array}{llll}u_{x 1} & u_{y 1} & u_{x 2} & u_{y 2}\end{array}\right\}$ o vetor que coleta os graus de liberdade dos nós 1 e 2 .

A construção da aproximação para os outros lados é análoga.

\subsubsection{Equacionamento}

Considere-se o elemento da Figura 3.2 submetido a forças de superfície $\overline{\boldsymbol{t}}_{c}$ em parte da fronteira estática $\Gamma_{\text {te }}$ e deslocamentos impostos $\bar{u}_{c}$ na fronteira cinemática $\Gamma_{\text {ue }}$. No equacionamento que se segue, a compatibilidade entre deformações no domínio e deslocamentos no contorno será expressa mediante o princípio dos trabalhos virtuais complementares (PTVC), enquanto o equilíbrio entre forças internas e externas será obtido via princípio dos trabalhos virtuais (PTV).

Enquanto o PTVC harmoniza os campos aproximativos de domínio e de contorno, o PTV assegura o equilíbrio de forma ponderada nas fronteiras estáticas. 


\subsubsection{Compatibilidade entre deformações no domínio e deslocamentos no contorno}

Pelo PTVC, na ausência de forças volúmicas, um estado compatível entre deformações e deslocamentos é possível se, para um sistema de forças virtuais externas $\delta \boldsymbol{t}_{\boldsymbol{c}}$ em equilíbrio com tensões virtuais $\delta \boldsymbol{\sigma}_{c}$, o trabalho virtual complementar interno realizado por $\delta \boldsymbol{\sigma}_{c}$ for igual ao trabalho virtual complementar externo realizado por $\delta \mathbf{t}_{\mathrm{c}}$. Considerando-se as deformações $\varepsilon_{c}$ em $\Omega_{\mathrm{e}}$ e os deslocamentos $\tilde{\boldsymbol{u}}$ em $\Gamma_{\mathrm{te}}$ e $\overline{\boldsymbol{u}}_{\boldsymbol{c}}$ em $\Gamma_{\mathrm{ue}}$, tem-se:

$$
\int_{\Omega_{e}} \delta \boldsymbol{\sigma}_{c}{ }^{T} \boldsymbol{\varepsilon}_{c} d \Omega=\int_{\Gamma_{t e}} \delta \boldsymbol{t}_{c}{ }^{T} \tilde{\boldsymbol{u}} d \Gamma+\int_{\Gamma_{u e}} \delta \boldsymbol{t}_{c}{ }^{T} \overline{\boldsymbol{u}}_{c} d \Gamma
$$

A partir das Eqs. (3.1) e (3.4) obtêm-se as respectivas variações $\delta \boldsymbol{\sigma}_{\boldsymbol{c}}=\boldsymbol{S}_{\boldsymbol{c}} \delta \boldsymbol{c}$ e $\delta \boldsymbol{t}_{\boldsymbol{c}}=\boldsymbol{T}_{c} \delta \boldsymbol{c}$, que levadas na equação anterior resulta:

$$
\delta c^{T} \int_{\Omega_{e}} S_{c}{ }^{T} \varepsilon_{c} d \Omega=\delta c^{T} \int_{\Gamma t e} T_{c}{ }^{T} \tilde{u} d \Gamma+\delta c^{T} \int_{\Gamma u e} T_{c}{ }^{T} \bar{u}_{c} d \Gamma, \forall \delta c
$$

Sendo $\delta \boldsymbol{c}$ arbitrário e aplicando-se a relação constitutiva (Eq. (2.5)), tem-se:

$$
\int_{\Omega_{e}} S_{c}^{T} f \sigma_{c} d \Omega=\int_{\Gamma_{t e}} T_{c}^{T} \tilde{u} d \Gamma+\int_{\Gamma_{u e}} T_{c}^{T} \bar{u}_{c} d \Gamma
$$

Inserindo-se as aproximações de $\sigma_{c}$ e $\tilde{\boldsymbol{u}}$, dadas respectivamente pelas Eqs. (3.1) e (3.5), encontra-se:

$$
F c-G d=e
$$

onde $\boldsymbol{F}=\int_{\Omega_{e}} \boldsymbol{S}_{c}{ }^{T} \boldsymbol{f} \boldsymbol{S}_{c} d \Omega, \boldsymbol{G}=\int_{\Gamma_{t e}} \boldsymbol{T}_{c}{ }^{T} \tilde{U} d \Gamma$ e $e=\int_{\Gamma_{u e}} T_{c}{ }^{T} \bar{u}_{c} d \Gamma$

Observa-se da Eq. (3.11) que a matriz $\boldsymbol{F}$ é simétrica. Além disso, em virtude do campo aproximado de tensões, na hipótese de forças volúmicas nulas, ser auto-equilibrado, pode-se demonstrar que sua integração no domínio $\Omega_{\mathrm{e}}$ pode ser realizada apenas no contorno $\Gamma_{\mathrm{e}}$.

De fato, a partir da expressão de $\boldsymbol{S}_{\boldsymbol{c}}$ dada pela Eq. (3.1) e sendo a matriz de rigidez a inversa da de flexibilidade, a expressão do integrando pode ser reescrita como:

$$
\boldsymbol{F}=\int_{\Omega_{e}} \boldsymbol{S}_{c}{ }^{T} f S_{c} d \Omega=\int_{\Omega_{e}} S_{c}{ }^{T} f D L_{c}{ }^{T} N_{c} d \Omega=\int_{\Omega_{e}} S_{c}{ }^{T} L_{c}{ }^{T} N_{c} d \Omega
$$

Aplicando-se, então, o teorema da divergência, vem:

$$
F=\int_{\Omega_{e}} S_{c}{ }^{T} L_{c}{ }^{T} N_{c} d \Omega=-\int_{\Omega_{e}}\left(L_{c} S_{c}\right)^{T} N_{c} d \Omega+\int_{\Gamma_{e}}\left(A S_{c}\right)^{T} N_{c} d \Gamma
$$


A partir da condição estabelecida pela Eq. (3.3) e da definição de $\boldsymbol{T}_{\boldsymbol{c}}$ dada pela Eq. (3.4), resulta:

$$
\boldsymbol{F}=\int_{\Gamma_{e}} T_{c}^{T} N_{c} d \Gamma
$$

Observa-se, na Eq. (3.14), que a matriz $\boldsymbol{F}$ é obtida do produto de matrizes que, em virtude das características da aproximação, apresentam colunas linearmente independentes. Segundo Freitas (1998), esta condição garante que ela seja não-singular e positiva definida.

\subsubsection{Equilíbrio entre forças internas e externas}

Pelo PTV, na ausência de forças volúmicas, um sistema está em equilíbrio sob ação de forças externas $\overline{\boldsymbol{t}}_{\boldsymbol{c}}$ se, para deslocamentos virtuais compatíveis $\delta \tilde{\boldsymbol{u}}$, isto é ao qual correspondem deformações virtuais $\delta \boldsymbol{\varepsilon}_{c}$, o trabalho virtual interno das tensões $\boldsymbol{\sigma}_{c}$ for igual ao trabalho virtual externo realizado por $\overline{\boldsymbol{t}}_{\boldsymbol{c}}$. Considerando-se deslocamentos virtuais $\delta \tilde{\boldsymbol{u}}$ em $\Gamma_{\mathrm{te}}$ e forças externas $\bar{t}_{c}$ em $\Gamma_{\text {te }}$, tem-se:

$$
\int_{\Omega_{e}} \delta \boldsymbol{\varepsilon}_{c}^{T} \boldsymbol{\sigma}_{c} d \Omega=\int_{\Gamma_{t e}} \delta \tilde{u} \bar{t}_{c} d \Gamma
$$

A forma equivalente da expressão anterior, considerando-se a aproximação de tensões $\boldsymbol{t}_{c}$ em $\Gamma_{\text {te }}$, é dada por:

$$
\int_{\Gamma_{t e}} \delta \tilde{\boldsymbol{u}}^{T} \boldsymbol{t}_{c} d \Gamma=\int_{\Gamma_{t e}} \delta \tilde{\boldsymbol{u}}^{T} \overline{\boldsymbol{t}}_{\boldsymbol{c}} d \Gamma
$$

A partir da Eq. (3.5) obtém-se a variação $\delta \tilde{\boldsymbol{u}}=\tilde{\boldsymbol{U}} \delta \boldsymbol{d}$ que levada à equação anterior resulta:

$$
\delta \boldsymbol{d}^{T} \int_{\Gamma_{t e}} \tilde{\boldsymbol{U}}^{T} \boldsymbol{t}_{c} d \Gamma=\delta \boldsymbol{d}^{T} \int_{\Gamma_{t e}} \tilde{\boldsymbol{U}}^{T} \overline{\boldsymbol{t}}_{c} \mathrm{~d} \Gamma
$$

Sendo $\delta \boldsymbol{d}$ arbitrário, tem-se:

$$
\int_{\Gamma_{t e}} \tilde{\boldsymbol{U}}^{T} \boldsymbol{t}_{c} \mathrm{~d} \Gamma=\int_{\Gamma_{t e}} \tilde{\boldsymbol{U}}^{T} \overline{\boldsymbol{t}}_{c} \mathrm{~d} \Gamma
$$

Finalmente, inserindo-se a aproximação de $\boldsymbol{t}_{c}$ dada pela Eq. (3.4) e considerando-se a definição da matriz $G$ dada pela Eq. (3.11), obtém-se:

$$
-\boldsymbol{G}^{T} \boldsymbol{c}=-\boldsymbol{q}
$$

onde $\boldsymbol{q}=\int_{\Gamma_{t e}} \tilde{\boldsymbol{U}}^{T} \overline{\boldsymbol{t}}_{c} \mathrm{~d} \Gamma$ 


\subsubsection{Sistema resolutivo}

O sistema resolutivo para o elemento é formado pelas Eqs. (3.11) e (3.19), sendo expresso por:

$$
\left[\begin{array}{cc}
\boldsymbol{F} & -\boldsymbol{G} \\
-\boldsymbol{G}^{T} & 0
\end{array}\right]\left\{\begin{array}{l}
\boldsymbol{c} \\
\boldsymbol{d}
\end{array}\right\}=\left\{\begin{array}{c}
\boldsymbol{e} \\
-\boldsymbol{q}
\end{array}\right\}
$$

O sistema representado na Eq. (3.20) é de formato idêntico ao analisado por Zienkiewicz et al. (1986) para formulações mistas, diferindo-se basicamente na definição das matrizes e vetores envolvidos. Nesse trabalho, encontra-se uma análise da condição necessária e suficiente para a existência e unicidade de sua solução. Tal análise é aqui reproduzida para a formulação híbrida-Trefftz.

A condição necessária, referida por condição de estabilidade, estabelece que a dimensão do vetor $\boldsymbol{c}$ não deve ser inferior à do vetor $\boldsymbol{d}$.

Para o entendimento desta condição, considere-se a matriz $\boldsymbol{F}$ de dimensões $\left(n_{c} \times n_{c}\right)$, a matriz $\boldsymbol{G}$ de dimensões $\left(n_{c} \times n_{d}\right)$, os vetores $\boldsymbol{c}$ e $\boldsymbol{e}$ de dimensão $\left(n_{c}\right)$ e os vetores $\boldsymbol{d}$ e $\boldsymbol{q}$ de dimensão $\left(n_{d}\right)$.

Sendo $\boldsymbol{F}$ não-singular, pode-se obter o vetor $\boldsymbol{c}$ diretamente da primeira linha do sistema:

$$
c=F^{-1} G d q+F^{-1} e
$$

Substituindo-se a expressão de $\boldsymbol{c}$ na segunda linha do sistema, obtém-se:

$$
K d=q-G^{T} F^{-1} e
$$

sendo $\boldsymbol{K}=\boldsymbol{G}^{T} \boldsymbol{F}^{-1} \boldsymbol{G}$.

Observa-se, da Eq. (3.22), que a existência e unicidade da solução do sistema resolutivo ficam condicionadas à matriz $\boldsymbol{K}$, especificamente em relação à sua singularidade. Considerando-se as dimensões envolvidas pode-se reescrevê-la como:

$$
\boldsymbol{K}=\boldsymbol{B}_{\left(n_{d} \times n_{C}\right)} \boldsymbol{G}_{\left(n_{C} \times n_{d}\right)}
$$

sendo $B=\boldsymbol{G}^{T} \boldsymbol{F}^{-1}$

Analisando-se as matrizes da Eq. (3.23), decorre de um teorema da álgebra linear (HOFFMAN; KUNZE, 1971), que a condição necessária para que $\boldsymbol{K}$ seja não-singular é que o número de colunas de $\boldsymbol{B}$ não seja inferior ao numero de colunas de $\boldsymbol{G}$. Assim sendo, tem-se a seguinte condição algébrica, doravante, denominada condição de estabilidade: 


$$
n_{c} \geq n_{d}
$$

Percebe-se que a condição de estabilidade estabelece que o número de graus de liberdade de tensão não deve ser inferior ao de deslocamentos.

Por sua vez, a condição suficiente somente se caracteriza com a positividade da matriz $\boldsymbol{K}$. Formalmente, tal matriz é positiva-definida se:

$$
\boldsymbol{d}^{T} K \boldsymbol{d}>0, \forall \boldsymbol{d} \neq \mathbf{0}
$$

Mas, sendo $\boldsymbol{K}=\boldsymbol{G}^{\boldsymbol{T}} \boldsymbol{F}^{-1} \boldsymbol{G}$, e $\boldsymbol{F}$ uma matriz positiva definida a condição dada pela Eq. (3.25), é equivalente a:

$$
G d \neq 0, \forall d \neq 0
$$

Analisando-se a Eq. (3.26), pode-se interpretá-la pela transformação linear:

$$
\mathrm{T}: \mathbb{R}^{n_{d}} \rightarrow \mathbb{R}^{n_{C}}, \text { tal que: } \mathrm{T}(\boldsymbol{d})=\boldsymbol{G d} \neq \mathbf{0}, \quad \forall \boldsymbol{d} \neq \mathbf{0}
$$

Segundo um teorema da álgebra linear (HOFFMAN; KUNZE, 1971), para que a Eq. (3.27) seja satisfeita a transformação linear deve ser injetora, tornando-se imperativas as seguintes condições:

- 1) $n_{c} \geq n_{d}$.

- 2) posto ( $n^{\circ}$ de colunas linearmente independentes) de $G$ igual a $n_{d}$.

Analisando-se a definição de $\boldsymbol{G}$, verifica-se que ambas as condições são atendidas. Na realidade, o atendimento à primeira implica no atendimento à segunda, já que as matrizes $\boldsymbol{T}_{c}$ e $\tilde{\boldsymbol{U}}$, envolvidas no calculo de $\boldsymbol{G}$, apresentam colunas linearmente independentes.

Assim sendo, percebe-se que a condição de estabilidade, quando da formulação híbrida-Trefftz, que utiliza funções linearmente independentes na construção das aproximações, é suficiente para garantir a existência e unicidade da solução do sistema resolvente expresso pela Eq. (3.20).

\subsubsection{Montagem do sistema global}

Evidentemente, o sistema resolutivo expresso pela Eq. (3.20), aplica-se apenas numa estrutura discretizada por um único elemento. Numa situação mais geral a discretização envolve uma rede de elementos. Neste contexto, o sistema global formado pela contribuição de cada elemento deve garantir a conectividade de toda rede e, assim, condições extras 
relacionadas à compatibilidade de deslocamentos e tensões, obrigam-se a estar presentes.

Em virtude das características da formulação, que aproxima diretamente deslocamentos no contorno, a compatibilidade de deslocamentos entre elementos que dividem uma fronteira comum é imposta em forma forte, isto é, adota-se a mesma aproximação para o campo de deslocamentos nas interfaces entre elementos. Para o estabelecimento desta condição, considerem-se dois elementos $i$ e $j$ quaisquer da discretização, cujos contornos são, respectivamente, denotados por $\Gamma_{\mathrm{e}}^{\mathrm{i}} \mathrm{e} \Gamma_{\mathrm{e}}{ }^{\mathrm{j}}$. Assim, na interface comum aos elementos $i$ e $j$, escreve-se:

$$
\tilde{\boldsymbol{u}}^{i}-\tilde{\boldsymbol{u}}^{j}=0, \mathrm{em} \Gamma_{\mathrm{e}}^{\mathrm{i}} \cap \Gamma_{\mathrm{e}}^{\mathrm{j}}
$$

Por outro lado, a condição referente à compatibilidade de tensões é imposta em forma fraca. Neste sentido, impõe-se o equilíbrio de forças nodais equivalentes em cada nó da discretização. Para um nó arbitrário $k$, vértice de $n_{\Gamma}$ fronteiras comuns entre elementos temse:

$$
\sum \boldsymbol{q}_{\sigma k l}=\sum \boldsymbol{q}_{\bar{t} k l} ; l=1, \cdots, n_{\Gamma}
$$

onde $\boldsymbol{q}_{\boldsymbol{\sigma} \boldsymbol{k} l}$ representam forças nodais equivalentes provenientes das tensões, enquanto $\boldsymbol{q}_{\bar{t} \boldsymbol{k} \boldsymbol{l}}$ representam forças nodais equivalentes provenientes do carregamento. Elas atuam num nó $k$, sendo calculadas para cada trecho I atrelado a ele.

Considerando-se que a rede adotada seja composta de $n$ elementos com um total de $n_{t}$ graus de liberdade em deslocamentos, o sistema global apresenta o aspecto:

$$
\left(\begin{array}{ccccc}
F_{1} & 0 & \cdots & 0 & \stackrel{-G_{1}}{1,2, \cdots, n_{t}} \\
0 & F_{2} & \cdots & 0 & -G_{2} \\
\vdots & \vdots & \ddots & \vdots & \vdots \\
0 & 0 & \cdots & F_{n} & -G_{n} \\
-G_{1}{ }^{T} & -G_{2}{ }^{T} & \cdots & -G_{n}{ }^{T} & 0
\end{array}\right)\left\{\begin{array}{c}
c_{1} \\
c_{2} \\
\vdots \\
c_{n} \\
d_{t}
\end{array}\right\}=\left\{\begin{array}{c}
e_{1} \\
e_{2} \\
\vdots \\
e_{n} \\
-q_{t}
\end{array}\right\}
$$

onde foram introduzidos o vetor $\boldsymbol{d}_{\boldsymbol{t}}$ que coleta os $n_{t}$ graus de liberdade nodais em deslocamentos e $\boldsymbol{q}_{\boldsymbol{t}}$ que pode ser interpretado como o vetor de forças nodais equivalentes.

Verifica-se que o equilíbrio ditado pela equação (3.29) é imposto no sistema pelo compartilhamento dos graus de liberdade nodais, mediante alocação das matrizes $\boldsymbol{G}_{\boldsymbol{i}}$ (mesma posição de colunas para cada grau de liberdade), mantendo-se, entretanto, o posicionamento das linhas correspondentes a cada elemento.

Para esclarecer todo o processo de montagem do sistema ditado pela Eq. (3.31), no tocante tanto à alocação de todas as matrizes e vetores envolvidos, quanto à compatibilidade 
de deslocamentos e à verificação ao equilíbrio de forças nodais equivalentes, apresenta-se no apêndice (C) um exemplo para uma chapa discretizada em dois elementos.

Ressalta-se que a existência e unicidade da solução do sistema global estão vinculadas à condição de estabilidade de cada elemento. Entretanto, em virtude do compartilhamento de graus de liberdade em deslocamentos, ela pode ser relaxada. Neste sentido aplica-se o teste do "mosaico", que consiste na verificação da Eq. (3.24) em todos os "mosaicos" que podem ser construídos com os elementos da discretização adotada (ZIENKIEWICZ et al. 1986). É importante destacar que a falha daquela condição num único "mosaico" compromete a estabilidade da solução global, mesmo quando a Eq. (3.24) é verificada para a totalidade de graus de liberdade envolvidos na análise.

\subsection{Elemento finito híbrido-Trefftz em domínios com orifício circular central}

Em domínios com orifícios circulares, em forma convencional, a discretização é caracterizada por um refino excessivo da rede e pela aproximação da geometria do furo. Entretanto, a formulação hibrida-Trefftz possibilita a inserção direta de elementos com orifício circular à rede.

Neste trabalho, para facilitar o processo de implementação, considera-se o elemento retangular de quatro nós, conforme ilustrado na Figura (3.3). Nele, a aproximação de tensões ditada pela Eq. (2.49) é particularizada para seu domínio, estabelecendo-se o seu centróide, como origem comum dos sistemas de coordenadas cartesiano e polar. A aproximação do campo de deslocamentos no contorno continua sendo dada pela Eq. (3.5).
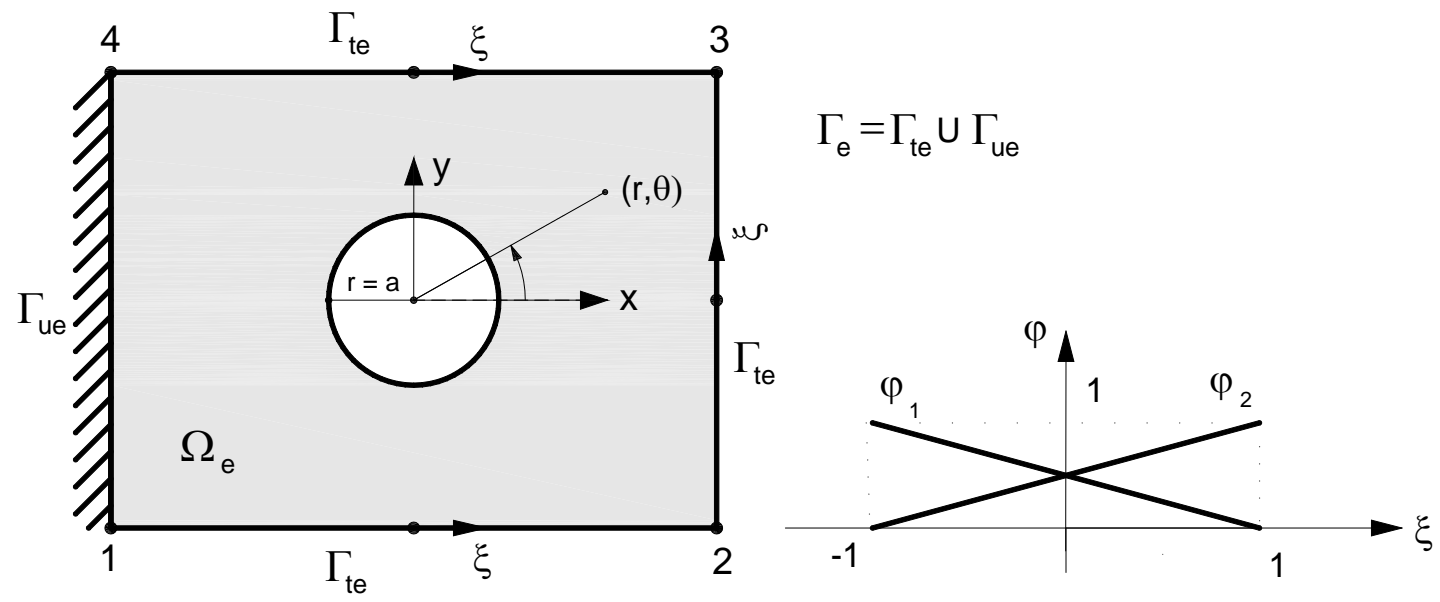

Figura 3.3. Elemento finito com orifício circular. 
Todo desenvolvimento realizado para elementos com domínio contínuo é naturalmente válido para elementos com orifício circular. Contudo, em virtude da adoção do sistema de coordenadas polares em $\Omega_{\mathrm{e}}$, algumas modificações são necessárias, no tocante às equações da Elasticidade e à aproximação de tensões. Em termos práticos, tais alterações são evidenciadas pelas novas expressões dos campos aproximados de tensões e das matrizes e vetores do sistema resolvente, quais sejam:

\section{- Aproximação de tensões no domínio}

A aproximação de tensões no domínio do elemento dada pela Eq. (2.49), tomada para um número conveniente de termos e particularizada para o domínio do elemento toma, doravante, a forma:

$$
\boldsymbol{\sigma}_{p}=\boldsymbol{S}_{p} \mathrm{c}, \mathrm{em} \Omega_{\mathrm{e}}
$$

\section{- Aproximação de tensões no contorno}

Por sua vez, a aproximação de tensões no contorno do elemento pode ser obtida da Eq. (3.32), quando do emprego da rotação de tensões, mediante a Eq. (2.8), e da aplicação do teorema de Cauchy (Eq. (2.10)), sendo expressa por:

$$
\boldsymbol{t}_{p}=\boldsymbol{T}_{p} \mathbf{c}, \text { em } \Gamma_{\mathrm{e}}
$$

sendo $\boldsymbol{T}_{p}=\boldsymbol{A} \boldsymbol{R}_{\sigma \theta} \boldsymbol{S}_{p}$.

\section{- Matrizes do sistema resolvente}

Considerando-se as equações da Elasticidade em coordenadas polares, a saber, equação de equilíbrio (Eq. (2.2)), equação de compatibilidade (Eq. (2.4)), relação constitutiva (Eq. (2.6)) e equação de Navier (Eq. (2.14)), além de outras auxiliares Eqs (2.7) e (2.10), o mesmo equacionamento realizado para elementos em domínios contínuos conduz às seguintes alterações nas expressões das matrizes do sistema resolvente:

$$
\begin{aligned}
& \boldsymbol{F}=\int_{\Gamma_{e}} \boldsymbol{T}_{\boldsymbol{p}}{ }^{\boldsymbol{T}} \boldsymbol{R}_{\boldsymbol{u} \boldsymbol{\theta}} \boldsymbol{N}_{\boldsymbol{p}} d \Gamma \\
& \boldsymbol{G}=\int_{\Gamma_{t e}} \boldsymbol{T}_{p}^{\boldsymbol{T}} \tilde{\boldsymbol{U}} d \Gamma
\end{aligned}
$$




$$
\boldsymbol{e}=\int_{\Gamma_{u e}} \boldsymbol{T}_{\boldsymbol{p}}{ }^{T} \bar{u}_{c} d \Gamma
$$

sendo $\boldsymbol{T}_{p}=\boldsymbol{A} \boldsymbol{R}_{\sigma \theta} \boldsymbol{S}_{p}$. 


\section{Formulação híbrida-Trefftz com enriquecimento seletivo em problemas bidimensionais da Elasticidade}

\subsection{Considerações iniciais}

No capítulo anterior, apresentou-se a formulação híbrida-Trefftz de forma simplificada, particularmente no tocante à aproximação do campo de deslocamentos no contorno do elemento. Ao contrário da aproximação do campo de tensões no domínio do elemento, a qual podia adquirir um número elevado de termos, a aproximação do campo de deslocamentos no contorno do elemento foi restrita apenas à combinação linear de funções de forma lineares.

Análises realizadas mediante tal formulação apresentam, em geral, uma reduzida capacidade de aproximação, requerendo, na maioria das vezes, uma discretização elevada. Verifica-se que o baixo grau das referidas funções de forma influi diretamente na qualidade da aproximação do campo de deslocamentos no contorno do elemento, uma vez que se subestimam distribuições não lineares. Além disto, o número reduzido de termos tomados nesta aproximação compromete também a qualidade da aproximação do campo de tensões, já que tanto o equilíbrio do elemento via PTV quanto à continuidade de tensões entre elementos são impostos em média, no sentido de Galerkin.

Assim sendo, com o intuito de melhorar a qualidade daquela formulação pode-se recorrer a técnicas de enriquecimento que influem diretamente na aproximação do campo de deslocamentos no contorno do elemento. Tais técnicas caracterizam-se pela ampliação da base de funções de aproximação.

No tocante às técnicas de enriquecimento duas opções são possíveis:

A primeira deriva do clássico refino-p. Ela consiste em construir uma nova base de funções de aproximação, ou base enriquecida, adicionando-se outra, de interesse, à existente.

A segunda é parte integrante do Método dos Elementos Finitos Generalizados, (DUARTE; BABUŠKA; ODEN, 2000). Tal técnica fundamenta-se nos seguintes requisitos, descritos de forma sintetizada:

- As funções de aproximação da base inicial devem constituir uma partição da 
unidade (PU), em essência funções cuja soma é unitária.

- A base enriquecida deve ser obtida pela união da PU com o resultado de seu produto por uma outra base de interesse.

- O enriquecimento deve ser realizado a partir de um domínio local de influência, denominado nuvem, formado pelo conjunto de elementos que apresentam um nó comum.

Neste trabalho, o enriquecimento será realizado combinando-se aspectos das duas técnicas descritas. Baseando-se na estrutura de enriquecimento centrada em nuvens, proposta pelo MEFG, adota-se o enriquecimento seletivo, o qual permite priorizar qualquer região de interesse no domínio estrutural $\Omega$, conforme ilustrado na Figura (4.1). Em cada nuvem, a aproximação do campo de deslocamentos nas fronteiras enriquecidas é construída a partir de uma base enriquecida, obtida pela união da base original com outra de interesse, como preconiza o clássico refino-p. Neste contexto novos graus de liberdade em tensão devem ser adicionados aos elementos enriquecidos, mediante um acréscimo de termos da aproximação do campo de tensões no domínio, de forma a satisfazer o teste do "mosaico" em toda a nuvem.

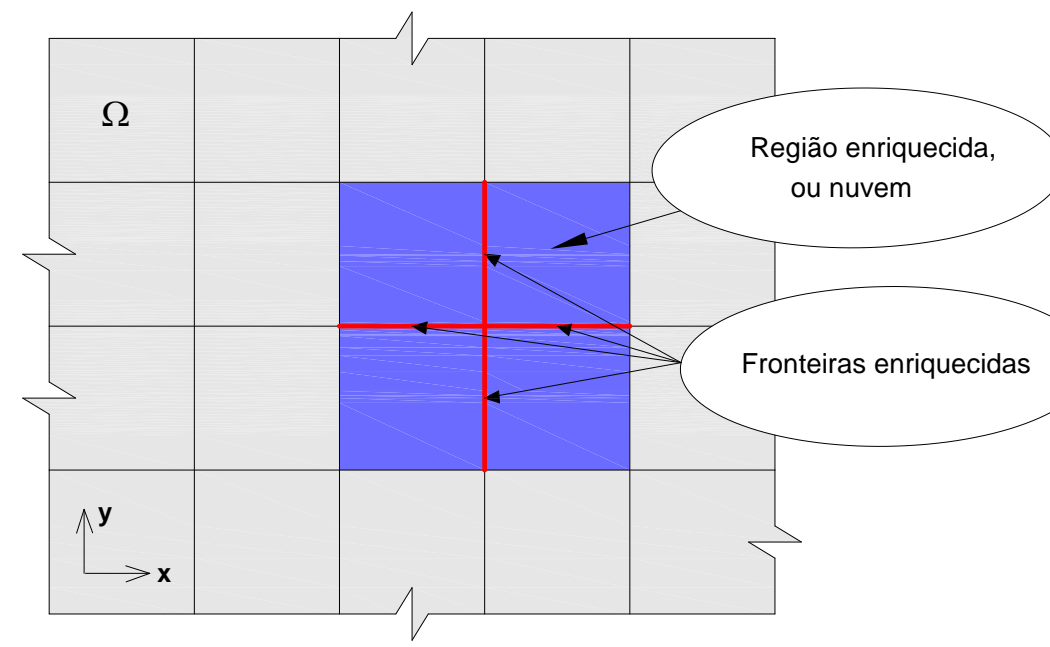

Figura 4.1. Nuvem de influência do enriquecimento seletivo.

Desta forma, neste capítulo, primeiramente, apresentam-se diferentes possibilidades de enriquecimento. Elas incluem bases de funções polinomiais com refinamento hierárquico e não hierárquico e ainda uma base não polinomial, que envolve funções trigonométricas. Em seguida, evidenciam-se as alterações decorrentes do enriquecimento no tocante tanto à aproximação do campo de deslocamentos no contorno do elemento, quanto às matrizes e vetores envolvidos na análise. Finalmente, estabelecem-se o novo formato do sistema resolvente para o elemento e os aspectos inerentes à montagem do sistema global da 
formulação híbrida-Trefftz com enriquecimento seletivo.

\subsection{Bases enriquecidas}

Conforme já mencionado, no estabelecimento das novas bases das funções de aproximação, adiciona-se à base inicial uma outra base de interesse. Evidentemente, a base adicionada deve ser formada por funções que apresentem boas propriedades de representação, isto é, capazes de captar as variações e taxas de variações de uma determinada distribuição do campo de deslocamentos no contorno do elemento. Além disso, é oportuno que tais funções sejam do tipo "bolha", ou seja, apresentem valores nulos nos nós. Tal característica preserva o significado físico dos graus de liberdade nodais iniciais.

Assim sendo, considere-se que a base original, formada pelas funções de forma $\varphi_{1}$ e $\varphi_{2}$ e utilizada para aproximar o campo de deslocamentos no contorno do elemento, tenha a seguinte representação:

$$
\beta_{0}=\left\{\varphi_{1}, \varphi_{2}\right\}
$$

Então, uma base enriquecida, genericamente denotada por $\beta_{\mathrm{g}}$, admite o aspecto:

$$
\beta_{g}=\left\{\varphi_{1}, \varphi_{2}\right\} \cup \beta_{E}
$$

sendo $\beta_{E}$ uma base de interesse utilizada no enriquecimento.

Considerando-se que $\beta_{E}$ seja composta de funções de aproximação enriquecedoras $h_{j}, j=1, . ., n$, todas elas distintas de $\varphi_{1}$ ou $\varphi_{2}$, a representação dada pela Eq. (4.2) é equivalente a:

$$
\beta_{g}=\left\{\varphi_{1}, \varphi_{2}, h_{1}, \cdots, h_{n}\right\}
$$

Neste trabalho, exploram-se três possibilidades de enriquecimento, apresentadas a seguir.

\section{- Enriquecimento polinomial com refinamento hierárquico}

No refinamento polinomial hierárquico a base enriquecida, denotada doravante por $\beta_{1}$ , é gerada pela seqüência:

- $\beta_{1}=\left\{\varphi_{1}, \varphi_{2}\right\} \cup\left\{\xi^{n-1}\left(1-\xi^{2}\right)\right\}$, com $n \geq 1$ e $n \in Z$

sendo $\mathrm{Z}$ o conjunto dos números inteiros.

Por exemplo, considerando-se os primeiros oito termos, $\beta_{1}$ apresenta o aspecto:

$$
\beta_{1}=\left\{(\xi+1) / 2,(-\xi+1) / 2,1-\xi^{2}, \xi\left(1-\xi^{2}\right), \xi^{2}\left(1-\xi^{2}\right), \xi^{3}\left(1-\xi^{2}\right), \xi^{4}\left(1-\xi^{2}\right), \xi^{5}\left(1-\xi^{2}\right)\right\}
$$


Denotando-se cada termo desta base por $m, m=1, \cdots, 8$, tem-se a representação indicada na Figura (4.2).

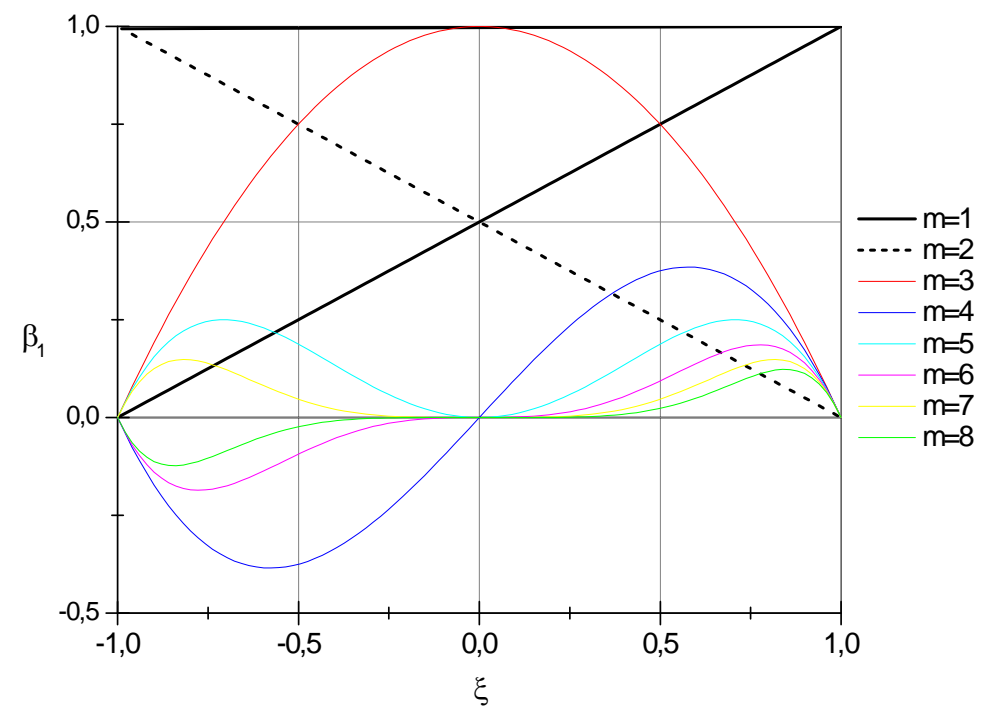

Figura 4.2. Base $\beta_{1}$, seqüência com oito termos.

\section{- Enriquecimento polinomial com refinamento não hierárquico}

Por sua vez, no refinamento polinomial não hierárquico a base enriquecida será denotada doravante por $\beta_{2}$. Ela é gerada pela seqüência:

- $\beta_{2}=\left\{\varphi_{1}, \varphi_{2}\right\} \cup\left\{(\xi+1)^{2 n}(\xi-1)^{2 n},(\xi+1)^{2 n-1}(\xi-1)^{2 n},-(\xi+1)^{2 n}(\xi-1)^{2 n-1}\right\}$, com $n \geq 1 \mathrm{e}$ $n \in \mathrm{Z}$

Por exemplo, considerando-se os primeiros oito termos, $\beta_{2}$ apresenta a forma:

$$
\begin{aligned}
\beta_{2}= & \left\{(\xi+1) / 2,(-\xi+1) / 2,(\xi+1)^{2}(\xi-1)^{2},(\xi+1)^{1}(\xi-1)^{2},-(\xi+1)^{2}(\xi-1)^{1},(\xi+1)^{4}(\xi-1)^{4},\right. \\
& \left.(\xi+1)^{3}(\xi-1)^{4},-(\xi+1)^{4}(\xi-1)^{3}\right\}
\end{aligned}
$$

Denotando-se seus termos por $m, m=1, \cdots, 8$, tem-se a representação da Figura (4.3):

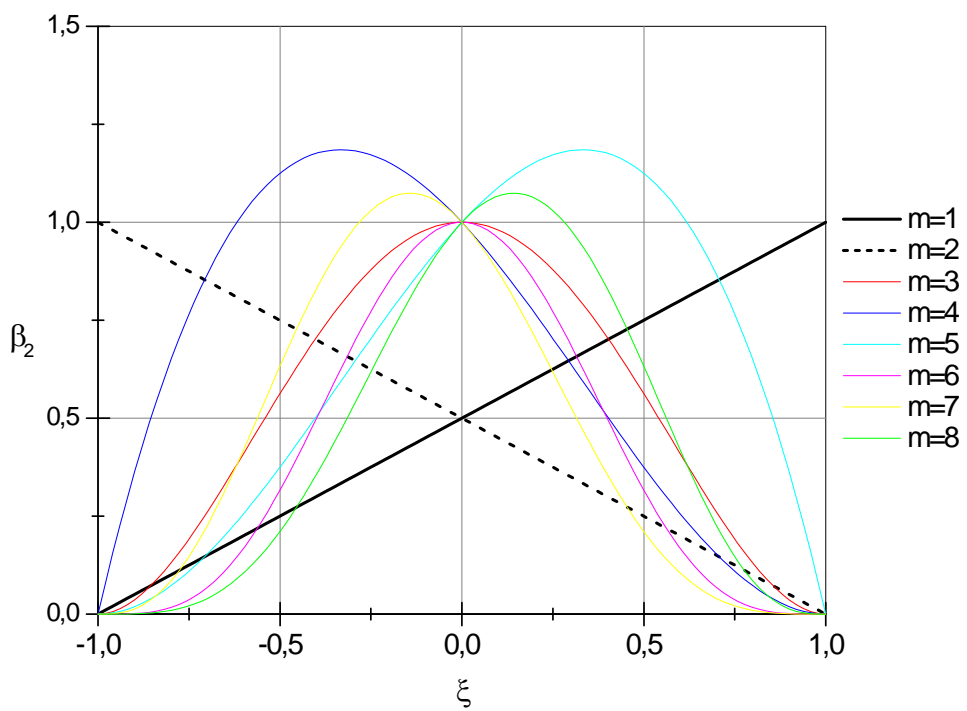

Figura 4.3. Base $\beta_{2}$, seqüência com oito termos. 


\section{- Enriquecimento não polinomial envolvendo funções trigonométricas}

Neste caso, a base enriquecida, doravante denotada por $\beta_{3}$, é gerada pela seqüência:

- $\beta_{3}=\left\{\varphi_{1}, \varphi_{2}\right\} \cup\left\{\left(1-\xi^{2}\right) \cos (n \xi),\left(1-\xi^{2}\right) \operatorname{sen}(n \xi)\right\}$, com $n \geq 1$ e $n \in Z$

Por exemplo, considerando-se os primeiros oito termos, $\beta_{3}$ apresenta o aspecto:

$$
\begin{aligned}
\beta_{3}= & \left\{(\xi+1) / 2,(-\xi+1) / 2,\left(1-\xi^{2}\right) \cos (\xi),\left(1-\xi^{2}\right) \operatorname{sen}(\xi),\left(1-\xi^{2}\right) \cos (2 \xi),\left(1-\xi^{2}\right) \operatorname{sen}(2 \xi),\right. \\
& \left.\left(1-\xi^{2}\right) \cos (3 \xi),\left(1-\xi^{2}\right) \operatorname{sen}(3 \xi)\right\}
\end{aligned}
$$

Denotando-se cada termo desta base por $m, m=1, \cdots, 8$, tem-se a representação indicada na Figura (4.4).

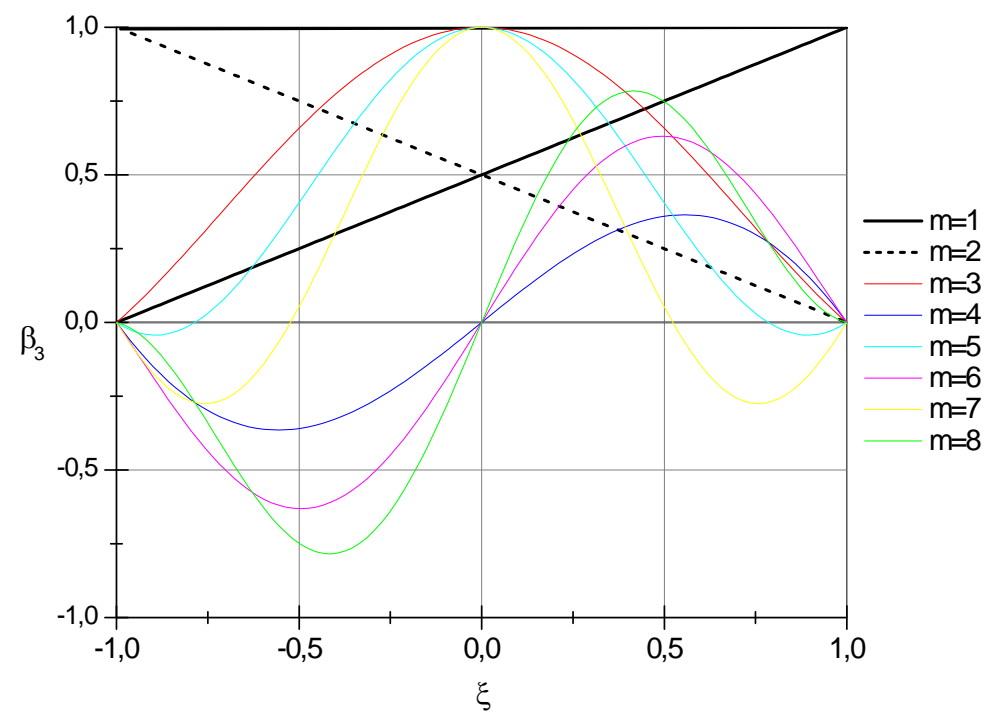

Figura 4.4. Base $\beta_{3}$, seqüência com oito termos.

\subsection{Aproximação do campo de deslocamentos numa fronteira enriquecida}

Considere-se que se pretenda enriquecer uma fronteira $\Gamma_{\mathrm{E}}$ de um determinado elemento, conforme ilustrado na Figura (4.5).

Então, deve-se acrescentar à aproximação inicial do campo de deslocamentos em $\Gamma_{\mathrm{E}}$ novos termos decorrentes do enriquecimento. Neste sentido, a nova aproximação do campo de deslocamentos em $\Gamma_{E}$ é obtida mediante combinação linear das funções de aproximação da base enriquecida, tomando-se como pesos os graus de liberdade em deslocamentos da referida fronteira. É importante destacar que os graus de liberdades acrescidos em virtude do enriquecimento não apresentam significado físico, mas os graus de liberdade nodais iniciais 
podem oportunamente manter seu significado, quando do emprego de funções de aproximação do tipo "bolha".

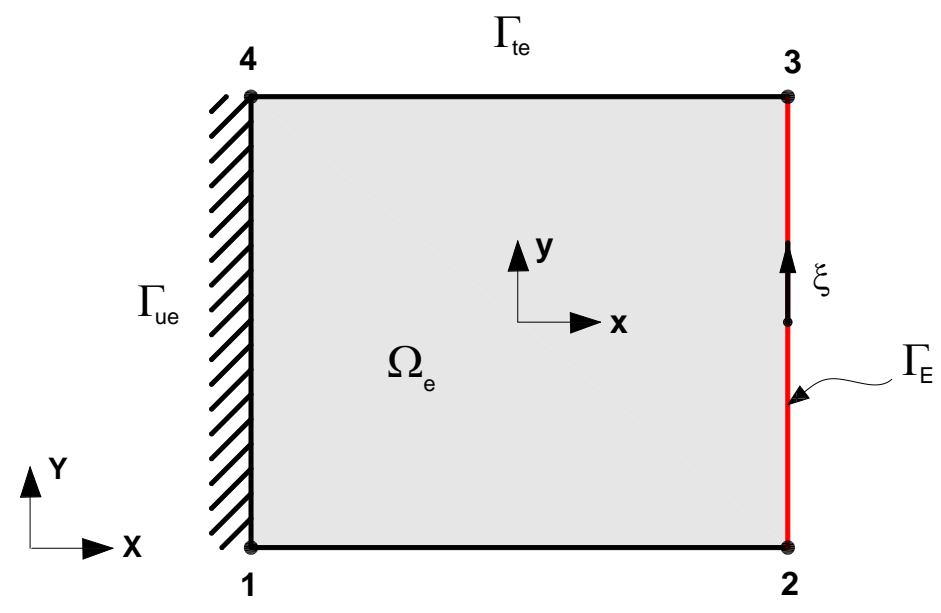

Figura 4.5. Fronteira enriquecida $\Gamma_{\mathrm{E}}$.

Assim sendo, considerando-se que o enriquecimento adicione um total de $\mathrm{n}$ graus de liberdade em deslocamentos pode-se representar a aproximação do campo de deslocamentos da fronteira enriquecida por:

$$
\tilde{\mathbf{u}}_{\mathrm{E}}=\tilde{\mathbf{U}}_{E} \mathbf{d}_{\mathrm{E}} ; \text { em } \Gamma_{\mathrm{E}} \subset \Gamma_{\text {te }}
$$

Para aproveitar a estrutura desenvolvida no capítulo anterior é oportuno representar a matriz $\tilde{\boldsymbol{U}}_{E}$ e o vetor $\boldsymbol{d}_{E}$ discriminando-se as parcelas referentes à aproximação inicial daquelas acrescidas por causa do enriquecimento, ou seja:

$$
\begin{aligned}
& \tilde{\boldsymbol{U}}_{E}=\left[\begin{array}{ll}
\tilde{\boldsymbol{U}}_{\Gamma_{E}} & \Delta \tilde{\boldsymbol{U}}_{\Gamma_{E}}
\end{array}\right] \\
& \boldsymbol{d}_{\boldsymbol{E}}=\left[\begin{array}{c}
\boldsymbol{d}_{\Gamma_{E}} \\
\Delta \boldsymbol{d}_{\Gamma_{E}}
\end{array}\right]
\end{aligned}
$$

O procedimento para obter a matriz $\tilde{\boldsymbol{U}}_{\Gamma_{E}}$ e do vetor $\boldsymbol{d}_{\Gamma_{E}}$, foi apresentado no item 3.2.2. Por outro lado, a matriz $\tilde{\boldsymbol{U}}_{\Gamma_{E}}$ e o vetor $\boldsymbol{d}_{\Gamma_{E}}$ foram introduzidos em virtude do enriquecimento, sendo apresentados no que se segue.

Considerando-se, uma base enriquecida genérica $\beta_{g}$ (vide Eq. (4.3)), para a matriz que coleta as funções que enriquecem a aproximação escreve-se:

$$
\tilde{\boldsymbol{U}}_{\Gamma_{E}}=\left[h_{1} \boldsymbol{I}_{2} \cdots h_{n} \boldsymbol{I}_{2}\right]
$$

sendo $h_{i}, i=1, \cdots, n$, funções utilizadas no enriquecimento da aproximação e $I_{2}$ a matriz identidade de ordem 2 .

Já o vetor que coleta os graus de liberdade acrescidos no enriquecimento é 
representado por:

$$
\Delta \boldsymbol{d}_{\Gamma_{E}}^{T}=\left\{\begin{array}{lllll}
\Delta u_{x 1} & \Delta u_{y 1} & \cdots & \Delta u_{x n} & \Delta u_{y n}
\end{array}\right\}
$$

sendo $\Delta u_{x i}$ e $\Delta u_{y i}, i=1, \cdots, n$, graus de liberdade decorrentes do enriquecimento nas direções $x$ e $y$, respectivamente.

\subsection{Equacionamento da formulação com enriquecimento}

Em virtude do enriquecimento evidenciam-se algumas alterações na formulação apresentada no capítulo anterior. Naturalmente, a nova aproximação do campo de deslocamentos numa fronteira enriquecida deve ser acompanhada pela inserção adequada de outras matrizes e vetores, exclusivamente decorrentes do enriquecimento, à estrutura inicial do sistema resolvente do elemento e consequentemente à do sistema resolvente global. Todo este procedimento é apresentado no que se segue.

\subsubsection{Matrizes e vetores acrescidos pelo enriquecimento}

Considere-se que após o enriquecimento a matriz $G$ referente à fronteira enriquecida $\Gamma_{E}$ (vide Fig. 4.5) seja denotada por $G_{E}$. Então, a partir da definição de $G$, a qual depende do sistema de coordenadas adotado (vide Eqs. (3.11) e (3.35)), escreve-se:

$$
\boldsymbol{G}_{E}=\int_{\Gamma_{E}} \boldsymbol{T}^{T} \tilde{\boldsymbol{U}}_{E} d \Gamma
$$

sendo $\boldsymbol{T}=\boldsymbol{T}_{c}$ ou $\boldsymbol{T}=\boldsymbol{T}_{p}$, segundo os sistemas cartesiano ou polar, respectivamente.

Inserindo-se a expressão da matriz $\tilde{\boldsymbol{U}}_{E}$ dada pela Eq. (4.5) na Eq. (4.9) resulta:

$$
\boldsymbol{G}_{E}=\left[\begin{array}{ll}
\boldsymbol{G}_{\Gamma_{E}} & \Delta \boldsymbol{G}_{\Gamma_{E}}
\end{array}\right]
$$

onde a matriz $G_{\Gamma_{E}}$ é parcela de $G$ referente à aproximação inicial, sendo dada por:

$$
G_{\Gamma_{E}}=\int_{\Gamma_{E}} \boldsymbol{T}^{T} \tilde{U}_{\Gamma_{E}} d \Gamma
$$

enquanto $\Delta \boldsymbol{G}_{\Gamma_{E}}$, introduzida em virtude do enriquecimento, é da forma: 


$$
\boldsymbol{G}_{\Gamma_{E}}=\int_{\Gamma_{E}} \boldsymbol{T}^{T} \tilde{\boldsymbol{U}}_{\Gamma_{E}} d \Gamma
$$

Analogamente, admitindo-se forças de superfície em $\Gamma_{E}$ e denotando-se por $\boldsymbol{q}_{E}$ a nova configuração do vetor $\boldsymbol{q}$ na referida fronteira, a partir da definição de $\boldsymbol{q}$ (vide Eq. (3.19)), tem-se:

$$
\boldsymbol{q}_{E}=\int_{\Gamma_{E}} \tilde{\boldsymbol{U}}_{E}{ }^{\top} \overline{\boldsymbol{t}}_{c} d \Gamma
$$

que é equivalente a:

$$
\boldsymbol{q}_{E}=\left[\begin{array}{ll}
\boldsymbol{q}_{\Gamma_{E}} & \boldsymbol{q}_{\Gamma_{E}}
\end{array}\right]
$$

onde o vetor $\boldsymbol{q}_{\Gamma_{E}}$ é a parcela das forças nodais equivalentes referente à aproximação inicial, sendo dado por:

$$
\boldsymbol{q}_{\Gamma_{E}}=\int_{\Gamma_{E}} \tilde{\boldsymbol{U}}_{\Gamma_{E}}{ }^{T} \overline{\boldsymbol{t}}_{\boldsymbol{c}} \mathrm{d} \Gamma
$$

Já o vetor $\Delta \boldsymbol{q}_{\Gamma_{E}}$, introduzido em função do enriquecimento, é da forma:

$$
\boldsymbol{q}_{\Gamma_{E}}=\int_{\Gamma_{E}} \tilde{\boldsymbol{U}}_{\Gamma_{E}}{ }^{T} \overline{\boldsymbol{t}}_{\boldsymbol{c}} d \Gamma
$$

\subsubsection{Sistema resolvente da formulação com enriquecimento}

A partir das Eqs. (4.6), (4.10) e (4.14), infere-se que o enriquecimento da fronteira $\Gamma_{\mathrm{E}}$ do elemento indicado na Fig. (4.5), insere a matriz $\Delta \boldsymbol{G}_{\Gamma_{E}}$ e os vetores $\boldsymbol{d}_{\Gamma_{E}}$ e $\Delta \boldsymbol{q}_{\Gamma_{E}}$ à estrutura inicial do sistema resolvente, que apresenta a seguinte configuração:

$$
\left[\begin{array}{ccc}
\boldsymbol{F} & -\boldsymbol{G} & -\Delta \boldsymbol{G}_{\Gamma_{E}} \\
-\boldsymbol{G}^{\boldsymbol{T}} & \boldsymbol{0} & \boldsymbol{0} \\
-\Delta \boldsymbol{G}_{\Gamma_{E}}{ }^{T} & \boldsymbol{0} & \boldsymbol{0}
\end{array}\right]\left\{\begin{array}{c}
\boldsymbol{c} \\
\boldsymbol{d} \\
\Delta \boldsymbol{d}_{\Gamma_{E}}
\end{array}\right\}=\left\{\begin{array}{c}
\boldsymbol{e} \\
-\boldsymbol{q} \\
-\boldsymbol{q}_{\Gamma_{E}}
\end{array}\right\}
$$

Evidentemente o sistema ditado pela Eq. (4.17) aplica-se num elemento com uma única fronteira enriquecida. Numa situação mais geral, podem-se enriquecer todas as fronteiras. Neste caso, deve-se acrescentar para cada fronteira enriquecida as matrizes e os vetores correspondentes.

Assim sendo, o sistema resolutivo para o elemento pode ser representado por: 


$$
\left[\begin{array}{ccc}
\boldsymbol{F} & -\boldsymbol{G} & -\Delta \boldsymbol{G} \\
-\boldsymbol{G}^{T} & \boldsymbol{0} & \boldsymbol{0} \\
-\Delta \boldsymbol{G}^{T} & \boldsymbol{0} & \boldsymbol{0}
\end{array}\right]\left\{\begin{array}{c}
\boldsymbol{c} \\
\boldsymbol{d} \\
\Delta \boldsymbol{d}
\end{array}\right\}=\left\{\begin{array}{c}
\boldsymbol{e} \\
-\boldsymbol{q} \\
-\boldsymbol{q}
\end{array}\right\}
$$

onde agora $\Delta \boldsymbol{d}$ representa o total de graus de liberdade acrescentados, $\Delta \boldsymbol{G}$ coleta as matrizes acrescidas e o vetor $\Delta \boldsymbol{q}$ os vetores acrescidos, considerando-se os possíveis trechos enriquecidos. Assim, as integrações de $\Delta \boldsymbol{G}$ e $\Delta \boldsymbol{q}$ passam a abranger toda a fronteira estática do elemento, ou seja:

$$
\boldsymbol{G}=\int_{\Gamma_{t e}} \boldsymbol{T}^{T} \tilde{\boldsymbol{U}} d \Gamma \text { e } \quad \boldsymbol{q}=\int_{\Gamma_{t e}} \tilde{\boldsymbol{U}}^{T} \overline{\boldsymbol{t}}_{\boldsymbol{c}} d \Gamma
$$

É importante salientar que a existência e unicidade do sistema resolvente ditado pela Eq. (4.18) continuam vinculados à condição de estabilidade (Eq. (3.24)). Porém, para verificação daquela condição, os graus de liberdade em deslocamentos devem incluir tanto os nodais quanto os provenientes do enriquecimento. Dessa forma, percebe-se que em virtude do enriquecimento um acréscimo de graus de liberdade em deslocamentos deve ser acompanhado por um aumento de graus de liberdade em tensões.

\subsubsection{Montagem do sistema resolvente global da formulação com enriquecimento}

Conforme já mencionado, na montagem do sistema global, condições extras referentes à compatibilidade de deslocamentos e de tensões devem ser impostas para garantir a conectividade de toda a rede de elementos da discretização adotada. Com este propósito, numa determinada fronteira enriquecida, deve-se considerar a nova aproximação do campo de deslocamentos.

Assim sendo, seja $\Gamma_{\mathrm{E}}$ uma fronteira enriquecida comum aos elementos $i$ e $j$ de um domínio $\Omega$, conforme ilustrado na Figura (4.6). Considerando-se que aproximação do campo de deslocamentos em $\Gamma_{E}$ para cada elemento seja dada pela Eq. (4.4), então a compatibilidade de deslocamentos, imposta em forma forte, é da forma:

$$
\tilde{\boldsymbol{u}}_{E}^{i}-\tilde{\boldsymbol{u}}_{E}^{j}=0, \text { em } \Gamma_{\mathrm{E}}
$$

Por sua vez, a compatibilidade de tensões é imposta em forma fraca. Considerando-se a matriz que coleta as funções de aproximação provenientes do enriquecimento dada pela Eq. (4.7) e que numa situação mais geral existam forças de superfície $\overline{\boldsymbol{t}}$ em $\Gamma_{\mathrm{E}}$ escreve-se: 


$$
\int_{\Gamma_{e}} \tilde{\boldsymbol{U}}_{\Gamma_{e}}^{T}\left(\boldsymbol{t}_{\boldsymbol{i}}+\boldsymbol{t}_{j}-\overline{\boldsymbol{t}}\right) d \Gamma=\boldsymbol{0}, \text { em } \Gamma_{\mathrm{E}}
$$

Ressalta-se que a Eq. (4.21) é uma condição complementar de equilíbrio. Ela verifica o equilíbrio de forças equivalentes na fronteira enriquecida $\Gamma_{\mathrm{E}} . \mathrm{O}$ equilíbrio de forças nodais equivalentes continua sendo imposto mediante a Eq. (3.29), em cada nó não restrito da discretização adotada.

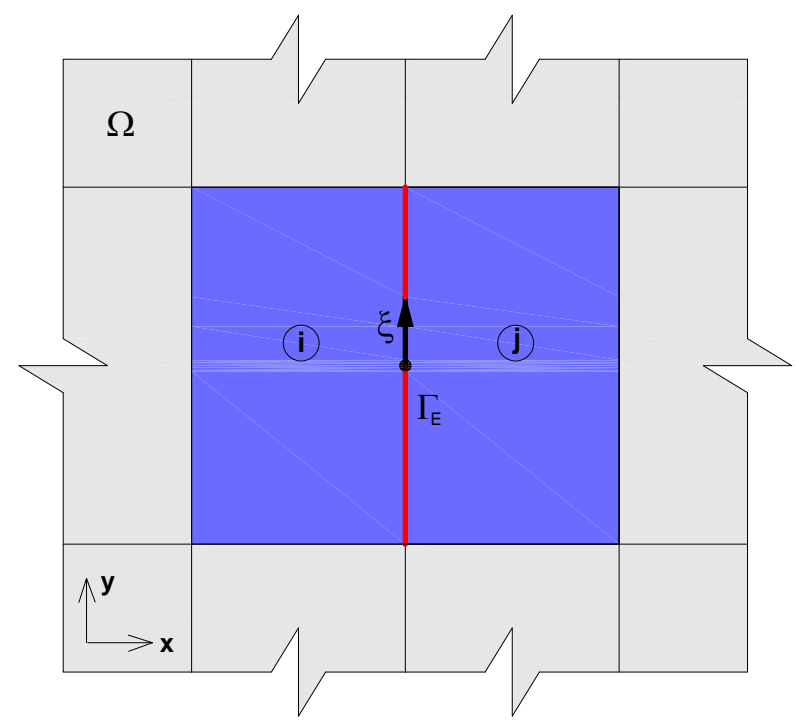

Figura 4.6. Fronteira enriquecida comum aos elementos $i$ e $j$.

Perante as considerações anteriores, admitindo-se uma rede composta de $n$ elementos com um total de $n_{t}$ graus de liberdade em deslocamentos nodais e $\Delta n_{t}$ graus de liberdade acrescidos em virtude do enriquecimento, o sistema global apresenta o aspecto genérico:

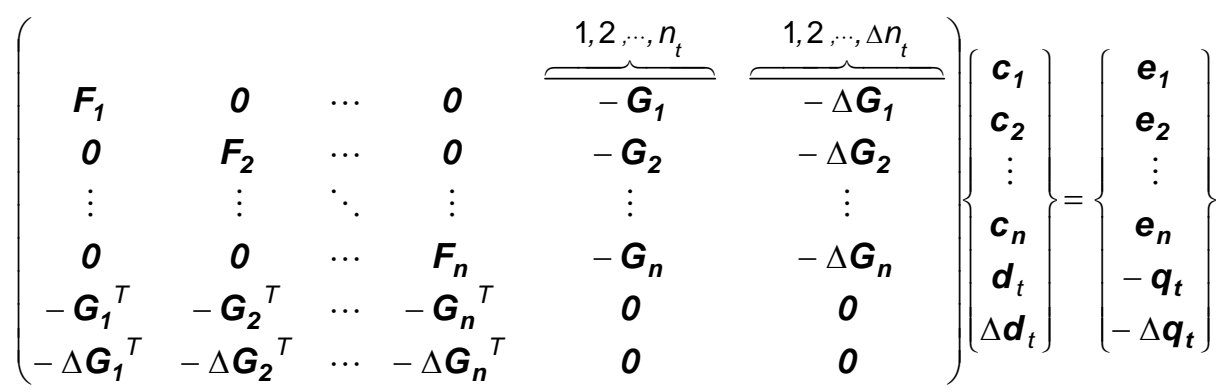

onde foram introduzidos o vetor $\Delta \boldsymbol{d}_{\boldsymbol{t}}$ que coleta os $\Delta n_{t}$ graus de liberdade em deslocamentos e o vetor $\Delta \boldsymbol{q}_{\boldsymbol{t}}$ de forças equivalentes das fronteiras enriquecidas.

Para que a condição dada pela equação (4.21) seja introduzida no sistema, as parcelas das matrizes $\Delta \boldsymbol{G}_{\boldsymbol{i}}$ e $\Delta \boldsymbol{G}_{j}$ referentes a uma fronteira enriquecida $\Gamma_{\mathrm{E}}$ devem ser alocadas nas mesmas colunas, mas nas linhas correspondentes ao posicionamento de cada elemento.

Para esclarecer todo o processo de montagem do sistema ditado pela Eq. (4.22), no 
tocante tanto à alocação de todas as matrizes e vetores envolvidos, quanto à compatibilidade de deslocamentos e à verificação ao equilíbrio de forças equivalentes, apresenta-se no apêndice (D) um exemplo para uma chapa discretizada em dois elementos.

Ressalta-se que a existência e unicidade da solução do sistema global estão vinculadas ao teste do "mosaico", cujos procedimentos foram descritos no item 3.2.3.4. Contudo, deve-se considerar que em cada possível "mosaico", construído com os elementos da discretização adotada, os graus de liberdade em deslocamentos compreendem tanto os nodais quanto os acrescidos pelo enriquecimento. 


\section{Método da Partição em formulação híbrida-Trefftz com enriquecimento seletivo aplicado a problemas com múltiplas fissuras em domínios bidimensionais}

\subsection{Considerações iniciais}

Neste capítulo aborda-se o Método da Partição em formulação híbrida-Trefftz com enriquecimento seletivo, particularmente na análise de problemas bidimensionais da Elasticidade cujos domínios envolvam múltiplas fissuras. São apresentadas as estratégias necessárias para a aplicação dos fundamentos original do método mediante a formulação apresentada no capítulo anterior.

Os fundamentos matemáticos do Método da Partição podem ser encontrados no trabalho de Babuška e Andersson (2005). Nele, encontra-se o equacionamento do método na simulação de um problema plano, regido pela equação de Laplace, cujo domínio envolva múltiplas fissuras, e sua generalização para o correspondente caso tridimensional; além de algumas aplicações numéricas do referido método via os procedimentos h-adaptativos e padaptativos do MEF.

Não é objetivo deste trabalho explorar as grandes potencialidades de aplicação do Método da Partição, as quais se evidenciam, sobretudo, em análises de maior complexidade no âmbito da mecânica do dano, confiabilidade estrutural, fadiga, entre outras. Desta forma, restringe-se a meta da análise à aproximação de algumas grandezas de interesse: campo de tensões, campo de deslocamentos e fatores de intensidade de tensão.

Assim sendo, primeiramente, apresentam-se neste capítulo os fundamentos do Método da Partição aplicado a problemas bidimensionais da Elasticidade em domínios com múltiplas fissuras. Neste sentido, são descritas as etapas do método para se aproximar os campos de tensões e de deslocamentos. Em seguida, abordam-se as alterações necessárias quando se emprega o referido método via formulação híbrida-Trefftz com enriquecimento seletivo. Por fim, descreve-se a técnica adotada na extração dos fatores de intensidade de tensão. 


\subsection{Fundamentos do Método da Partição}

No estabelecimento dos fundamentos do Método da Partição aplicado a problemas bidimensionais da Elasticidade, considera-se o PVC definido no item 2.2. Contudo, estende-se sua formulação admitindo-se a presença de múltiplas fissuras $f_{i}$ em $\Omega$, conforme ilustrado na Figura (5.1).

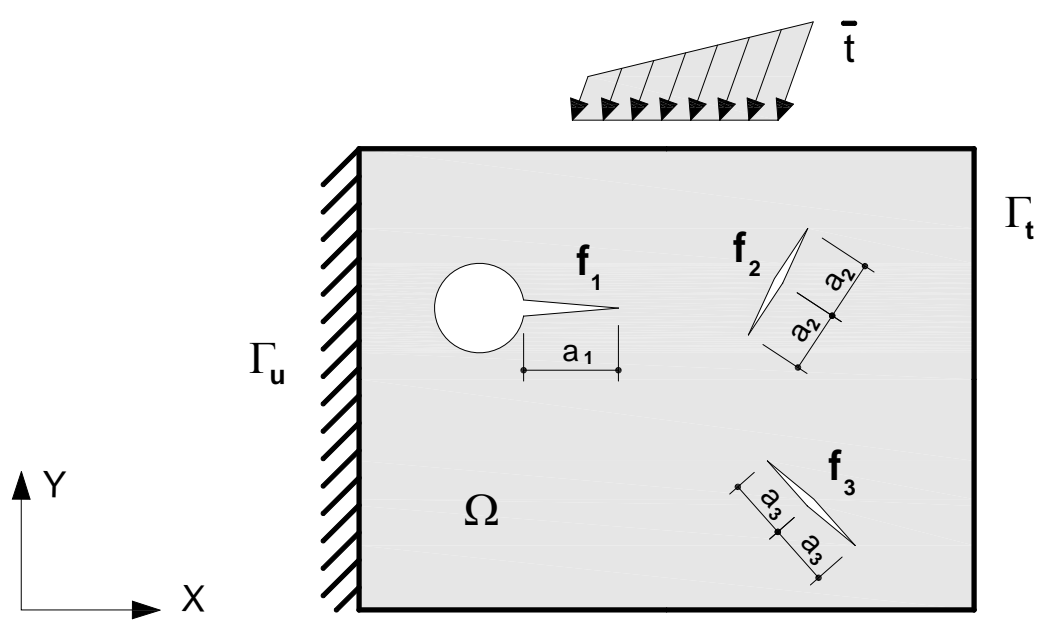

Figura 5.1. Sólido com múltiplas fissuras.

O Método da Partição consiste em analisar o referido PVC mediante a sobreposição adequada de uma série de subproblemas, assim designados: problema global $\left(\boldsymbol{P} \boldsymbol{G}_{\boldsymbol{0}}\right)$, problemas locais $\left(\boldsymbol{P} \boldsymbol{L}_{\boldsymbol{k}}\right)$ e problemas globais $\left(\boldsymbol{P} \boldsymbol{G}_{\boldsymbol{k}}\right)$.

Admite-se que a solução do campo de deslocamentos do PVC seja determinada pela sobreposição das soluções $\boldsymbol{u}_{\boldsymbol{0}}$ de $\boldsymbol{P} \boldsymbol{G}_{\boldsymbol{o}}, \boldsymbol{u}_{\boldsymbol{L} \boldsymbol{k}}$ de cada $\boldsymbol{P \boldsymbol { L } _ { \boldsymbol { k } }}$ e $\boldsymbol{u}_{G \boldsymbol{k}}$ de cada $\boldsymbol{P G _ { \boldsymbol { k } }}$. Assim, segundo o sistema de coordenadas cartesianas, escreve-se:

$$
\boldsymbol{u}=\boldsymbol{u}_{\boldsymbol{0}}+\sum_{k=1}^{n_{p}} \alpha_{k} \boldsymbol{u}_{G \boldsymbol{k}}-\sum_{k=1}^{n_{p}} \alpha_{k} \boldsymbol{u}_{L \boldsymbol{k}}, \mathrm{em} \Omega
$$

Na Eq. (5.1), o número de termos $n_{p}$ depende do número de fissuras existentes e da quantidade de problemas locais considerados para cada uma delas, enquanto os fatores de escala $\alpha_{k}, k=1, \cdots, n_{p}$, são obtidos da imposição, em forma fraca, da nulidade de tensões nas faces de cada fissura $f_{i}$, conforme será exposto adiante.

Por sua vez, o campo de tensões pode ser obtido a partir da Eq. (5.1) utilizando-se a relação de compatibilidade (Eq. 2.3) e a relação constitutiva (Eq. 2.5), sendo representado por: 


$$
\boldsymbol{\sigma}=\boldsymbol{\sigma}_{\boldsymbol{o}}+\sum_{k=1}^{n_{p}} \alpha_{k} \boldsymbol{\sigma}_{G \boldsymbol{k}}-\sum_{k=1}^{n_{p}} \alpha_{k} \boldsymbol{\sigma}_{L \boldsymbol{k}}, \text { em } \Omega
$$

sendo $\boldsymbol{\sigma}_{\boldsymbol{0}}=\boldsymbol{D} \boldsymbol{L}_{\boldsymbol{c}}{ }^{T} \boldsymbol{u}_{\boldsymbol{0}}, \boldsymbol{\sigma}_{G k}=\boldsymbol{D} \boldsymbol{L}_{\boldsymbol{c}}{ }^{T} \boldsymbol{u}_{G k}$ e $\boldsymbol{\sigma}_{L k}=\boldsymbol{D} \boldsymbol{L}_{\boldsymbol{c}}{ }^{T} \boldsymbol{u}_{L k}$.

As peculiaridades de cada subproblema e seus objetivos são descritos a seguir.

\subsubsection{Problema global $\left(\mathrm{PG}_{0}\right)$}

No problema global $P G_{0}$, considera-se o PVC sem as fissuras $f_{i}$ e mantêm-se todas as condições de contorno, conforme ilustrado na Figura (5.2). A meta da análise é obter a distribuição de tensões nas linhas $\mathbf{L}_{\mathbf{f i}}$ correspondentes a cada uma das fissuras previamente desconsideradas.

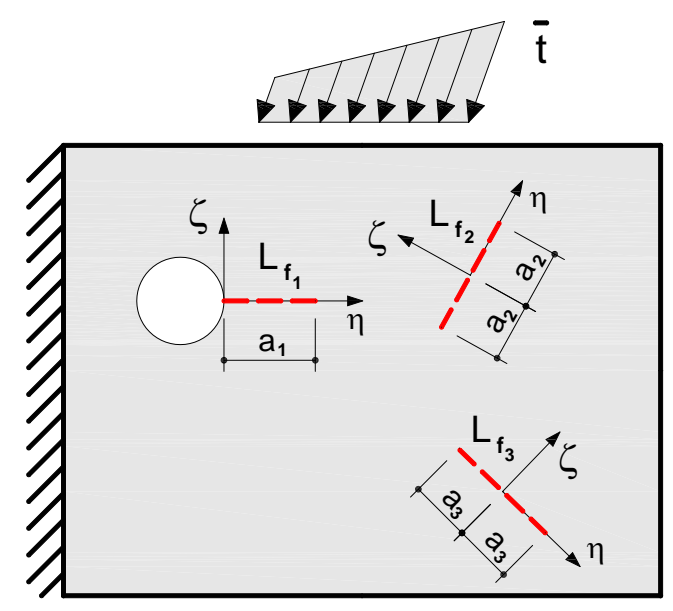

Figura 5.2. Problema global $P G_{0}$.

Em cada linha, determina-se um vetor resultante de tensão representado por:

$$
\boldsymbol{t}_{\boldsymbol{0}}{ }^{T}=\left\{\begin{array}{ll}
t_{0 \eta} & t_{0 \zeta}
\end{array}\right\}, \mathrm{em} \mathrm{\mathbf {L } _ { \mathrm { fi } }}
$$

onde $t_{0 \eta}$ e $t_{0 \zeta}$ são as componentes da resultante de tensão na direção da linha e perpendicular a ela, respectivamente; $\xi$ e $\eta$ são eixos de referência posicionados nas faces das fissuras.

\subsubsection{Problemas locais $\left(\mathrm{PL}_{\mathrm{k}}\right)$}

Para o estabelecimento dos problemas locais $P \boldsymbol{L}_{\boldsymbol{k}}$, consideram-se, individualmente, domínios locais arbitrários de influência $\boldsymbol{V}_{f i}$, cada qual englobando uma fissura $f_{i}$ do 
problema. As condições de contorno em cada $\boldsymbol{V}_{f i}$ podem ser arbitrárias, mas devem garantir o equilíbrio estático em qualquer situação de carregamento. Em cada $\boldsymbol{V}_{\boldsymbol{f i}}$ define-se um caminho interno de referência $\Gamma_{\mathrm{fi}}$ no qual se insere toda a fissura $f_{i}$, conforme ilustrado na Figura
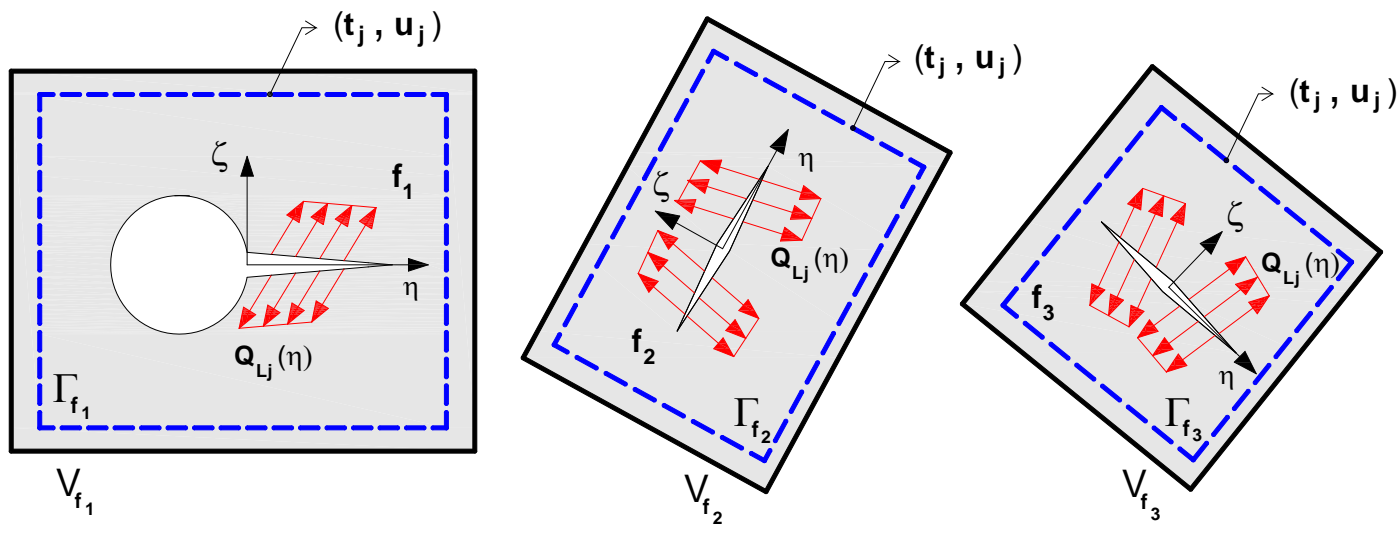

Figura 5.3. Problemas locais $\mathrm{PL}_{k}$.

Cada problema local caracteriza-se pela aplicação de um carregamento fictício $\boldsymbol{Q}_{\boldsymbol{L}_{j}}$ nas faces de uma fissura envolvida por um $\boldsymbol{V}_{\boldsymbol{f i}}$. Assim, para cada fissura podem-se estabelecer inúmeros problemas locais.

O objetivo de cada problema local é determinar, para um carregamento fictício $\boldsymbol{Q}_{\boldsymbol{L}_{\boldsymbol{j}}}$ aplicado sobre as faces de uma fissura $f_{i}$, as correspondentes distribuições de tensão $\boldsymbol{t}_{\boldsymbol{j}}$ e deslocamentos $\boldsymbol{u}_{j}$ ao longo do respectivo caminho $\Gamma_{\mathrm{fi}}$ (vide Figura (5.3)).

A distribuição das tensões resultantes nas faces de cada fissura $f_{i}$ pode ser obtida pela contribuição dos correspondentes problemas locais. Para isto, considera-se que cada carregamento fictício seja multiplicado por um fator de escala $\alpha_{j}$. Assim, considerando-se um total de $n$ carregamentos fictícios aplicados sobre as faces de $f_{i}$, tem-se:

$$
\boldsymbol{t}_{L}=\sum_{j=1}^{n} \alpha_{j} \boldsymbol{Q}_{L j}=\boldsymbol{Q}_{\mathbf{L}} \boldsymbol{\alpha}
$$

Na Eq. (5.4), o vetor $\alpha$ é formado pelos fatores de escala $\alpha_{j}$, sendo representado por:

$$
\boldsymbol{\alpha}^{T}=\left\{\begin{array}{lllll}
\alpha_{1} & \cdots & \alpha_{j} & \cdots & \alpha_{n}
\end{array}\right\}
$$

Por sua vez, a matriz $\boldsymbol{Q}_{L}$, constituída pelos vetores $\boldsymbol{Q}_{L_{j}}$, toma a forma:

$$
\text { - } \boldsymbol{Q}_{\boldsymbol{L}}=\left[\begin{array}{lllll}
\boldsymbol{Q}_{\boldsymbol{L} 1} & \cdots & \boldsymbol{Q}_{\boldsymbol{L} j} & \cdots & \boldsymbol{Q}_{L_{n}}
\end{array}\right] ; \text { onde } \boldsymbol{Q}_{\boldsymbol{L} j}{ }^{T}=\left\{\begin{array}{ll}
\boldsymbol{Q}_{L j \eta} & Q_{L j \zeta}
\end{array}\right\} \text {, para } j=1, \cdots, n,
$$


sendo $Q_{L j \eta}$ e $Q_{L j \zeta}$ componentes dos carregamentos fíctícios nas direções $\eta$ e $\zeta$, respectivamente.

\subsubsection{Problemas globais $\left(\mathrm{PG}_{\mathrm{k}}\right)$}

Nos problemas globais $P G_{k}$, considera-se o PVC sem fissuras e mantêm-se somente as condições de contorno homogêneas em forças e em deslocamentos. Cada $P G_{\boldsymbol{k}}$ caracterizase pela aplicação das distribuições de tensões $\boldsymbol{t}_{\boldsymbol{j}}$ e deslocamentos $\boldsymbol{u}_{\boldsymbol{j}}$ provenientes de um $\boldsymbol{P} \boldsymbol{L}_{\boldsymbol{k}}$, que atuam sobre um caminho $\Gamma_{\mathrm{fi}}$ previamente definido, no correspondente $\boldsymbol{P} \boldsymbol{G}_{\boldsymbol{k}}$, conforme ilustrado na Figura (5.4). Portanto, a cada $\boldsymbol{P} \boldsymbol{L}_{\boldsymbol{k}}$ corresponde um $\boldsymbol{P} \boldsymbol{G}_{\boldsymbol{k}}$.

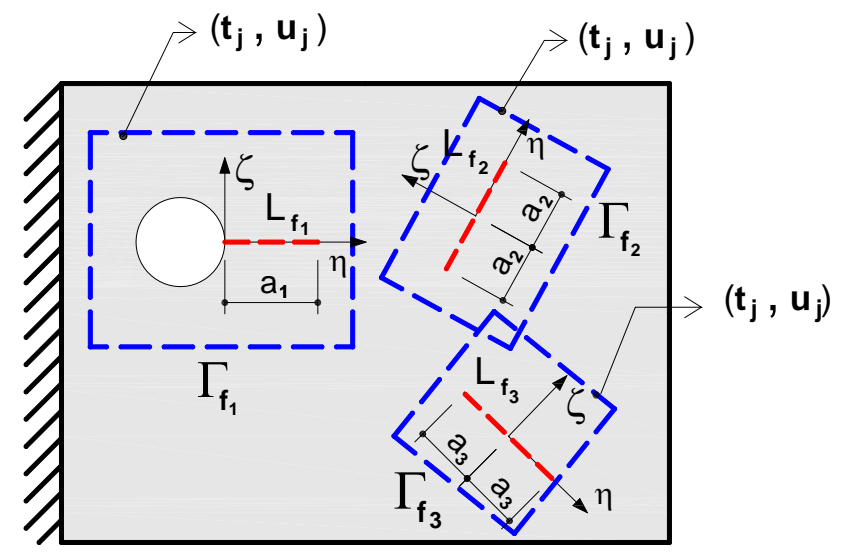

Figura 5.4. Problemas globais $P G_{k}$.

A meta de cada $P G_{k}$ é obter a distribuição de tensões nas linhas $\mathbf{L}_{\mathrm{fi}}$ correspondentes a cada uma das fissuras previamente desconsideradas.

Em cada linha, a distribuição de tensões resultante pode ser obtida pela contribuição das tensões provenientes de cada $\boldsymbol{P} \boldsymbol{G}_{\boldsymbol{k}}$. Neste sentido, para estabelecer uma relação de proporcionalidade de tensões entre os problemas locais e globais, consideram-se os mesmos fatores de escala $\alpha_{j}$ utilizados na Eq. (5.4), para cada uma das fissuras $f_{i}$. Assim, na linha correspondente a uma fissura $f_{i}$, da sobreposição de todos os $n_{p}$ problemas globais $P G_{k}$, escreve-se:

$$
\boldsymbol{t}_{G}=\sum_{k=1}^{n p} \alpha_{k} \boldsymbol{Q}_{G k}=\boldsymbol{Q}_{G} \boldsymbol{\alpha}, \mathrm{em} \mathbf{L}_{\mathbf{f i}}
$$

Na Eq. (5.5) a matriz $\boldsymbol{Q}_{G}$, constituída pelos vetores $\boldsymbol{Q}_{G_{k}}$, e o vetor $\boldsymbol{\alpha}$ admitem a 
forma:

$$
\begin{aligned}
& \boldsymbol{Q}_{G}=\left[\begin{array}{lllll}
\boldsymbol{Q}_{G_{1}} & \cdots & \boldsymbol{Q}_{G_{k}} & \cdots & \boldsymbol{Q}_{G_{n p}}
\end{array}\right] ; \text { onde } \boldsymbol{Q}_{k}{ }^{T}=\left\{\begin{array}{lll}
Q_{G k n} & Q_{G k \zeta}
\end{array}\right\} \text {, sendo } Q_{G_{k n}} \text { e } \\
& Q_{G_{k \zeta}} \text {, para } k=1, \cdots, n_{p} \text {, componentes das tensões na linha nas direções } \eta \text { e } \\
& \zeta, \text { respectivamente. } \\
& \boldsymbol{\alpha}^{T}=\left\{\begin{array}{lllll}
\alpha_{1} & \cdots & \alpha_{k} & \cdots & \alpha_{n p}
\end{array}\right\}
\end{aligned}
$$

Observa-se que agora o vetor $\boldsymbol{\alpha}$ deve ser formado pela totalidade de fatores de escala $\alpha_{k}$ de todos os problemas locais, oriundos de todas as fissuras, de forma a captar a influência de cada uma das fissuras sobre as demais.

\subsubsection{Solução numérica do método}

Segundo o Método da Partição, a distribuição de tensões em uma fissura $f_{i}$ do PVC é

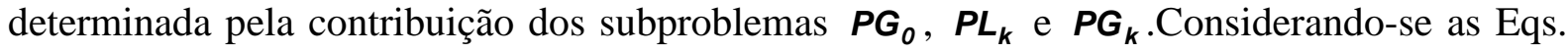
(5.3), (5.4) e (5.5), admite-se que a distribuição de tensões seja da forma:

$$
\boldsymbol{t}=\boldsymbol{t}_{\boldsymbol{0}}+\left(\boldsymbol{Q}_{G}-\boldsymbol{Q}_{L}\right) \boldsymbol{\alpha}
$$

Os fatores de escala da Eq. (5.6) podem ser determinados impondo-se, em forma fraca, a nulidade de tensões ao longo do comprimento da referida fissura. Assim, tomando-se como ponderação a primeira variação da aproximação ditada pela Eq. (5.4), escreve-se

$$
\int_{L_{f i}} \delta \boldsymbol{t}_{L}^{T}\left[\boldsymbol{t}_{\boldsymbol{0}}+\left(\boldsymbol{Q}_{G}-\boldsymbol{Q}_{L}\right) \boldsymbol{\alpha}\right] d L=\mathbf{0}
$$

que é equivalente a:

$$
\delta \boldsymbol{\alpha}^{T} \int_{L_{f i}} \boldsymbol{Q}_{L}^{T}\left[\boldsymbol{t}_{\boldsymbol{0}}+\left(\boldsymbol{Q}_{G}-\boldsymbol{Q}_{L}\right) \boldsymbol{\alpha}\right] d L=\boldsymbol{O}, \forall \delta \boldsymbol{\alpha}
$$

Sendo $\delta \boldsymbol{\alpha}$ arbitrário resulta:

$$
K_{p i} \alpha=-v_{p i}
$$

onde $\boldsymbol{K}_{p i}=\int_{L_{f i}} \boldsymbol{Q}_{L}^{T}\left(\boldsymbol{Q}_{G}-\boldsymbol{Q}_{L}\right) d L$ e $\boldsymbol{v}_{p i}=\int_{L_{f i}} \boldsymbol{Q}_{L}^{T} \boldsymbol{t}_{0} d L$

É importante ressaltar que na Eq. (5.9) o vetor $\alpha$ é formado pelos fatores $\alpha_{k}$ provenientes de todos os problemas locais. Além disso, para que a operação de subtração entre as matrizes $\boldsymbol{Q}_{L}$ e $\boldsymbol{Q}_{\boldsymbol{G}}$ definida pela matriz $\boldsymbol{K}_{\boldsymbol{p} i}$ seja possível, a matriz $\boldsymbol{Q}_{L}$, definida na Eq. (5.4), deve ter agora a mesma dimensão de $\boldsymbol{Q}_{G}$, bastando alocar as posições originais 
conforme os fatores de escala $\alpha_{k}$ e preencher os espaços restantes com entradas nulas.

A totalidade de fatores de escala determinados a partir do sistema formado pelo uso sistemático da Eq. (5.9) para todas as fissuras constituem os fatores $\alpha_{k}$ utilizados para determinar a solução do campo de deslocamentos do PVC (Eq. (5.1)).

\subsection{Equacionamento do Método da Partição em formulação híbrida- Trefftz com enriquecimento seletivo}

Neste item abordam-se as particularidades do Método da Partição em formulação híbrida-Trefftz com enriquecimento seletivo, conforme descrito a seguir.

\subsubsection{Aproximação dos campos de deslocamentos e tensões}

$\mathrm{Na}$ aplicação do Método da Partição em formulação híbrida-Trefftz com enriquecimento seletivo, admite-se que a solução do campo de deslocamentos do PVC seja semelhante à Eq. (5.1), porém restrita ao contorno dos elementos da discretização adotada. Por conseguinte, para cada elemento a aproximação do campo de deslocamentos, resultado da sobreposição dos subproblemas $P G_{0}, P L_{k}$ e $P G_{k}$, fica com o aspecto:

$$
\tilde{\boldsymbol{u}}=\tilde{\boldsymbol{u}}_{0}+\sum_{k=1}^{n_{p}} \alpha_{k} \tilde{\boldsymbol{u}}_{G \boldsymbol{k}}-\sum_{k=1}^{n_{p}} \alpha_{k} \tilde{\boldsymbol{u}}_{L \boldsymbol{k}}, \text { em } \Gamma_{\text {te }}
$$

Analogamente, admite-se que a aproximação do campo de tensões do PVC seja dada pela Eq. (5.2), agora restrita ao domínio de cada elemento da discretização adotada, ou seja:

$$
\boldsymbol{\sigma}=\boldsymbol{\sigma}_{\boldsymbol{o}}+\sum_{k=1}^{n_{p}} \alpha_{k} \boldsymbol{\sigma}_{G \boldsymbol{k}}-\sum_{k=1}^{n_{p}} \alpha_{k} \boldsymbol{\sigma}_{L \boldsymbol{k}}, \text { em } \Omega_{\mathrm{e}}
$$

\subsubsection{Análise do problema global $\left(\mathrm{PG}_{0}\right)$}

$\mathrm{Na}$ análise do problema global aproveita-se toda a estrutura da formulação híbridaTrefftz com enriquecimento seletivo. Adotando-se uma discretização adequada aproximam-se as grandezas de interesse do $\mathbf{P G}_{0}$ : campo de tensões, campo de deslocamentos e distribuição 
de tensões nas fronteiras dos elementos pertencentes às linhas correspondentes às fissuras previamente desconsideradas.

Desta forma, a aproximação do campo de tensões de cada elemento é ditada pela Eq. (3.1), sendo representada por:

$$
\boldsymbol{\sigma}_{0}=\boldsymbol{S}_{c} \mathbf{c}, \mathrm{em} \Omega_{\mathrm{e}}
$$

Por sua vez, a aproximação básica do campo de deslocamento na fronteira estática é dada pela Eq. (3.5), admitindo a forma:

$$
\tilde{\mathbf{u}}_{\mathbf{0}}=\tilde{\mathbf{U}} \mathbf{d} ; \mathrm{em} \Gamma_{\text {te }}
$$

Em particular, numa fronteira enriquecida, deve-se substituir a aproximação básica pela aproximação ditada pela Eq. (4.4), ou seja:

$$
\tilde{\mathbf{u}}_{\mathbf{0}}=\tilde{\mathbf{U}}_{E} \mathbf{d}_{\mathrm{E}} ; \text { em } \Gamma_{\mathrm{E}} \subset \Gamma_{\mathrm{te}}
$$

Finalmente, a distribuição de tensões na linha $L_{f i}$ correspondente a uma fissura desconsiderada previamente pode ser determinada aplicando-se primeiramente o teorema de Cauchy à Eq. (5.12) e em seguida transferindo as tensões calculadas para o sistema local de coordenadas da referida linha, conforme indicado na Figura (5.5).
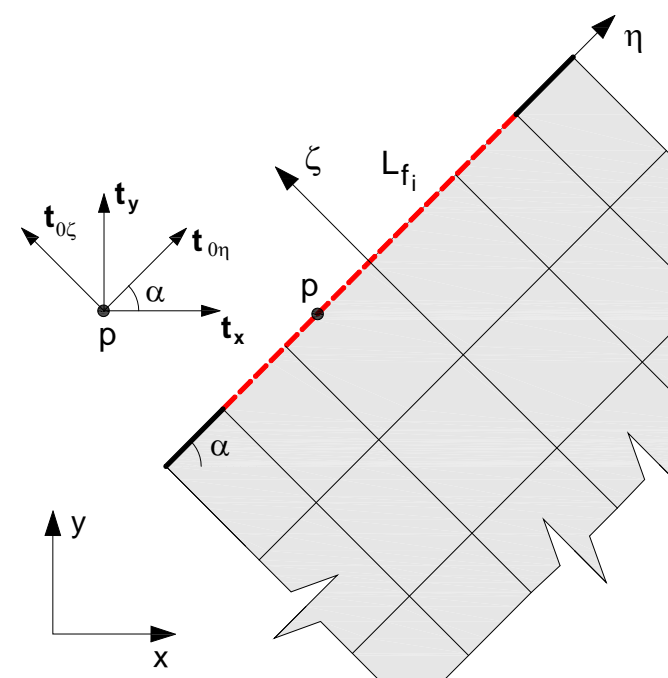

Figura 5.5. Tensões associadas a um ponto de $\mathrm{L}_{\mathrm{fi}}$.

Assim sendo, nos elementos que apresentam uma fronteira pertencente a $\mathbf{L}_{\mathrm{fi}}$, tem-se:

$$
\boldsymbol{t}_{\boldsymbol{0}}=\boldsymbol{R}_{\alpha} \boldsymbol{t}, \text { em } \Gamma_{\mathrm{te}} \cap \mathbf{L}_{\mathrm{fi}}
$$

onde $\boldsymbol{t}_{\boldsymbol{0}}{ }^{T}=\left\{\begin{array}{ll}t_{0 \eta} & t_{0 \zeta}\end{array}\right\}$, sendo $t_{0 \eta}$ e $t_{0 \zeta}$ as componentes da tensão na direção da linha e perpendicular a ela, respectivamente; $\boldsymbol{R}_{\alpha}=\left(\begin{array}{cc}\cos \alpha & \operatorname{sen} \alpha \\ -\operatorname{sen} \alpha & \cos \alpha\end{array}\right)$ é a matriz de rotação e $\boldsymbol{t}=\boldsymbol{A} \boldsymbol{\sigma}_{\boldsymbol{0}}$. 


\subsubsection{Análise dos problemas locais $\left(\mathrm{PL}_{\mathrm{k}}\right)$}

$\mathrm{Na}$ análise dos problemas locais, a formulação híbrida-Trefftz com enriquecimento seletivo é utilizada de maneira sistemática mediante a aplicação de uma série de carregamentos fictícios sobre as faces de cada fissura $f_{i}$ do problema. A partir de uma discretização conveniente, para cada $\mathbf{P L}_{\mathbf{k}}$, caracterizado por um carregamento fíctício, aproximam-se as grandezas de interesse: campo de tensões, campo de deslocamentos e a distribuição de tensões e deslocamentos no caminho $\Gamma_{\mathrm{fi}}$ referente a uma fissura $f_{i}$.

Para aplicação dos carregamentos fictícios, estabelece-se para cada fissura um sistema local de coordenadas adimensionais cuja origem depende das características da fissura. Numa fissura completamente inserida no domínio, adota-se como origem o ponto eqüidistante de suas extremidades, enquanto numa fissura de bordo, adota-se o ponto da extremidade livre, conforme ilustrado na Figura (5.6).

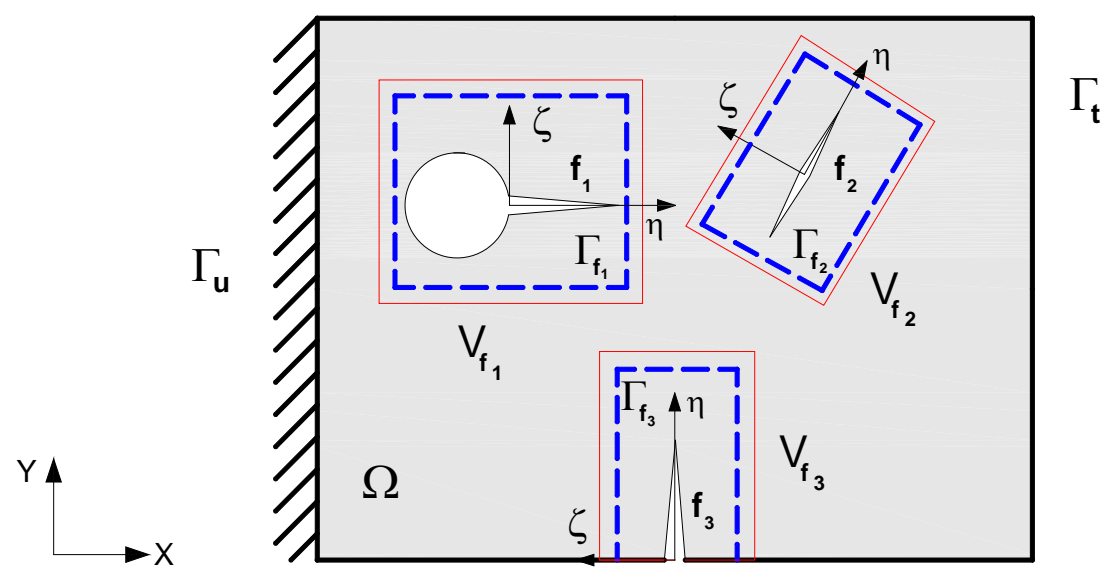

Figura 5.6. Sistema de coordenadas locais em cada fissura.

A série de carregamentos fictícios é aplicada alternadamente segundo as direções $\zeta$ e $\eta$ mediante um sistema auto-equilibrado de forças atuantes nas faces de cada fissura $f_{i}$, conforme ilustrado na Figura (5.7). 


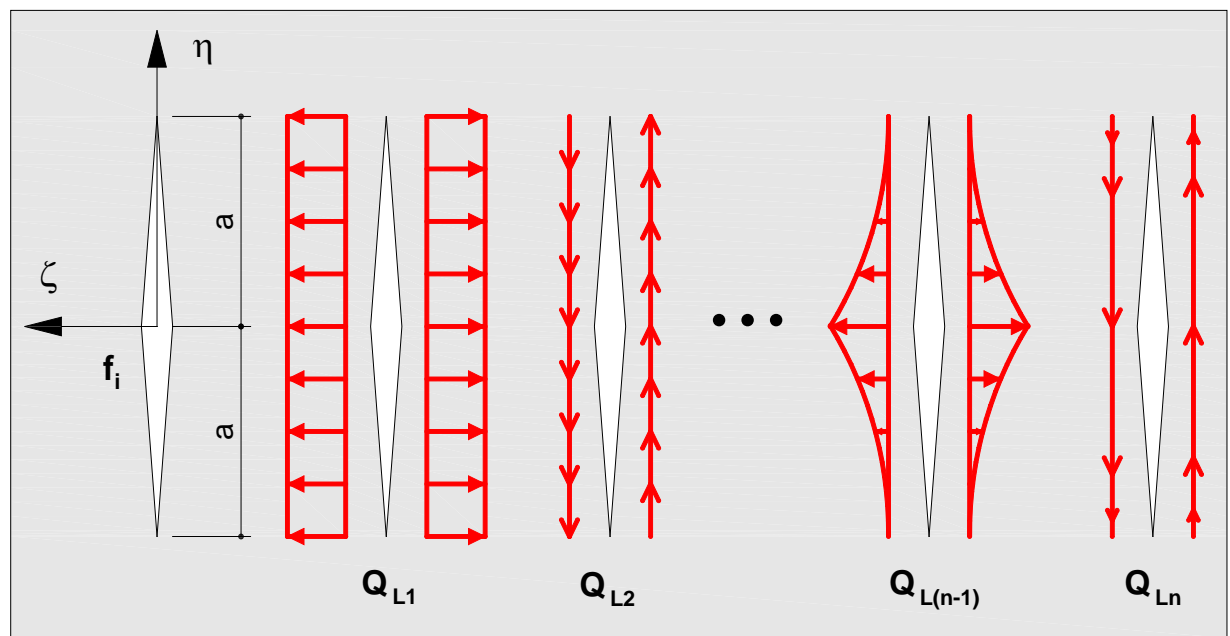

Figura 5.7. Série de carregamentos fictícios em uma fissura $f_{i}$.

Em ambas as direções adota-se uma sequiência hierárquica de carregamentos fictícios em função da coordenada adimensional $\eta$. Considerando-se que em cada direção sejam aplicados $n_{q}$ carregamentos fictícios, a distribuição da intensidade das forças fictícias atuantes nas faces da referida fissura tanto na direção $\zeta$ quanto na direção $\eta$, destas seqüências, pode ser obtido do conjunto de funções:

$$
g_{i}(\eta)=(1-|\eta|)^{i-1}, i=1, \cdots, n_{q}
$$

sendo $\eta=u$ / $a$, onde a representa o comprimento efetivo da fissura e $u$ é uma variável que depende de suas características. Numa fissura completamente inserida no domínio $u \in[-a, a]$, enquanto numa fissura de bordo $u \in[0, a]$.

É importante destacar que a Eq. (5.16) não é restrita a um método numérico específico e eventualmente pode sofrer algumas alterações. Em particular, quando de seu emprego mediante a formulação híbrida-Trefftz, deve-se relacionar o sistema de coordenadas adimensionais de cada fissura com o de seus elementos adjacentes, tal que o conjunto de funções $g_{i}$ seja função das coordenadas adimensionais $\xi$ dos elementos. Assim sendo, considere-se uma fissura $f_{i}$ de comprimento efetivo $a$, à qual esteja vinculada um conjunto de elementos, conforme ilustrado na Figura (5.8). 


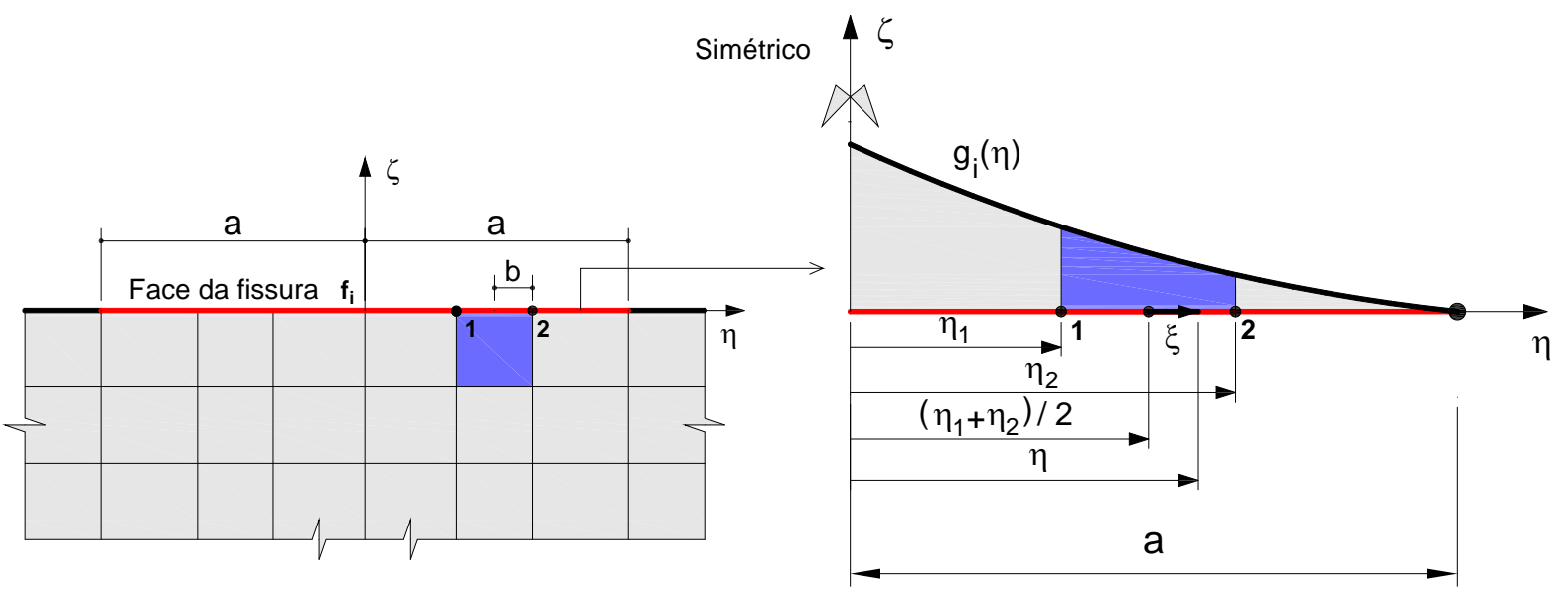

Figura 5.8. Distribuição das forças fictícias numa das faces de uma fissura.

Então, considerando-se um elemento adjacente a uma das faces da fissura, a partir de aspectos geométricos obtêm-se a seguinte relação entre as coordenadas dos sistemas locais da fissura e do elemento:

$$
\eta=\frac{\eta_{1}+\eta_{2}}{2}+\frac{b}{a} \xi
$$

sendo $\eta_{1}$ e $\eta_{2}$ valores adimensionais especificados para o sistema coordenado da fissura, referentes aos nós 1 e 2 , respectivamente e $b$ a metade do lado do elemento pertencente a uma das faces da fissura (vide Figura (5.8)).

Por conseguinte, o conjunto de funções representativo da distribuição de intensidade das forças fictícias dado pela Eq. (5.16) tem a forma:

$$
g_{i}(\xi)=\left(1-\left|\frac{\eta_{1}+\eta_{2}}{2}+\frac{b}{a} \xi\right|\right)^{i-1} ; \text { para } i=1, \cdots, n_{q}
$$

A partir das considerações anteriores, verifica-se que a distribuição de tensões resultantes nas faces da referida fissura é composta de $2 n_{q}$ termos e fica com o aspecto:

$$
\boldsymbol{t}_{L}=\sum_{j=1}^{2 n_{q}} \alpha_{j} \boldsymbol{Q}_{L j}=\boldsymbol{Q}_{L} \boldsymbol{\alpha}
$$

Todas as grandezas envolvidas na Eq. (5.19) já foram apresentadas genericamente no item 5.2.2 (vide Eq. (5.4)). Contudo, em virtude da disposição adotada na aplicação da série de carregamentos fictícios, algumas grandezas passam a ter representações especificas. A partir do conjunto de funções dado pela Eq. (5.18), verifica-se que os vetores $\boldsymbol{Q}_{L j}$ são da forma:

$$
\text { - } \boldsymbol{Q}_{L 1}=\left\{\begin{array}{c}
g_{1} \\
0
\end{array}\right\}, \boldsymbol{Q}_{\mathbf{L} 2}=\left\{\begin{array}{c}
0 \\
g_{1}
\end{array}\right\}, \ldots, \boldsymbol{Q}_{L(n-1)}=\left\{\begin{array}{c}
g_{n q} \\
0
\end{array}\right\}, \boldsymbol{Q}_{\mathbf{L n}}=\left\{\begin{array}{c}
0 \\
g_{n q}
\end{array}\right\}
$$


e, por conseguinte, a matriz $\mathbf{Q}_{\mathbf{L}}$ apresenta o aspecto:

- $\boldsymbol{Q}_{\mathbf{L}}=\left[\begin{array}{lll}g_{1} \boldsymbol{I}_{2} & \cdots & g_{n_{q}} \boldsymbol{I}_{2}\end{array}\right] ;$ onde $\boldsymbol{I}_{2}$ é a matriz identidade de ordem 2.

A análise dos problemas locais completa-se com a determinação dos campos de tensões no domínio dos elementos, deslocamentos nas fronteiras estáticas e distribuições de tensões e deslocamentos nos caminhos $\Gamma_{\mathrm{fi}}$ de cada fissura $f_{i}$.

Assim sendo, para cada $\mathbf{P L}_{\mathbf{k}}$, caracterizado pela aplicação de um $\boldsymbol{Q}_{\mathbf{L} j}$, a aproximação do campo de tensões em cada elemento é ditada pela Eq. (3.1), sendo representada por:

$$
\sigma_{L k}=S_{c} c, \text { em } \Omega_{e}
$$

enquanto, a aproximação básica do campo de deslocamentos na fronteira estática dos elementos, para cada $\mathbf{P L}_{\mathbf{k}}$, é dada pela Eq. (3.5), admitindo o aspecto:

$$
\tilde{\boldsymbol{u}}_{L \boldsymbol{k}}=\tilde{\mathbf{U}} \mathbf{d} ; \mathrm{em} \Gamma_{\mathrm{te}}
$$

Em particular, numa fronteira enriquecida, deve-se substituir a aproximação básica pela aproximação ditada pela Eq. (4.4), ou seja:

$$
\tilde{\boldsymbol{u}}_{L \boldsymbol{k}}=\tilde{\mathbf{U}}_{E} \mathbf{d}_{\mathrm{E}} ; \text { em } \Gamma_{\mathrm{E}} \subset \Gamma_{\text {te }}
$$

Por sua vez, a distribuição de tensões em um caminho $\Gamma_{\mathrm{fi}}$ pode ser obtida de maneira sistemática mediante o teorema de Cauchy para cada problema local considerado na respectiva fissura $f_{i}$. Desta forma, determina-se a distribuição de tensões para cada termo da série de carregamentos fictícios $\left(j=1, \cdots, 2 n_{q}\right)$, a partir da relação:

$$
\boldsymbol{t}_{\boldsymbol{j}}=\boldsymbol{A} \boldsymbol{\sigma}_{\Gamma \boldsymbol{j}}, \mathrm{em} \Gamma_{\mathrm{fi}}
$$

onde se introduziu o vetor $\sigma_{\Gamma j}$ que representa a distribuição de tensões dos elementos adjacentes a um caminho $\Gamma_{\mathrm{fi}}$.

Finalmente, na determinação dos deslocamentos em $\Gamma_{\mathrm{fi}}$, utiliza-se a Eq. (3.5) ou a Eq. (4.4) sistematicamente para cada termo da série de carregamentos fictícios $\left(j=1, \cdots, 2 n_{q}\right)$ numa fissura, ou seja:

$$
\tilde{u}_{j}=\tilde{U} \mathbf{d}, \text { em } \Gamma_{\mathrm{fi}}
$$

$\mathrm{ou}$

$$
\tilde{\mathbf{u}}_{j}=\tilde{\mathbf{U}}_{E} \mathbf{d}_{\mathbf{E}}, \text { em } \Gamma_{\mathrm{fi}}
$$




\subsubsection{Análise dos problemas globais $\left(\mathrm{PG}_{\mathrm{k}}\right)$}

$\mathrm{Na}$ análise dos problemas globais a formulação híbrida-Trefftz com enriquecimento seletivo é utilizada de forma ordenada. Aplicam-se sistematicamente as distribuições de tensões $\boldsymbol{t}_{\boldsymbol{j}}$ e deslocamentos $\boldsymbol{u}_{\boldsymbol{j}}$, provenientes de cada $\mathrm{PL}_{\mathbf{k}}$, sobre o caminho $\Gamma_{\mathrm{fi}}$ do correspondente $\mathbf{P G}_{\mathbf{k}}$. Adotando-se uma discretização adequada, para cada $\mathbf{P G _ { \mathbf { k } }}$, aproximamse as grandezas de interesse: campo de tensões, campo de deslocamentos e distribuição de tensões nas linhas correspondentes às fissuras previamente desconsideradas.

Assim sendo, na análise de cada $\mathbf{P G}_{\mathbf{k}}$ mediante a formulação híbrida-Trefftz com enriquecimento seletivo, devem-se inserir as referidas distribuições $\boldsymbol{t}_{\boldsymbol{j}}$ e $\boldsymbol{u}_{\boldsymbol{j}}$ na estrutura do sistema resolutivo, em substituição aos tradicionais valores prescritos de forças de superfície $\overline{\boldsymbol{t}}_{\boldsymbol{c}}$ e deslocamentos $\overline{\boldsymbol{u}}_{\boldsymbol{c}}$. Por conseguinte, os vetores $\boldsymbol{q}, \Delta \boldsymbol{q}$ e $\boldsymbol{e}$ presentes no sistema ditado pela Eq. (4.18), adquirem o aspecto:

$$
\begin{gathered}
\boldsymbol{q}=\int_{\Gamma_{f i}} \tilde{\boldsymbol{U}}^{\boldsymbol{T}} \boldsymbol{t}_{j} d \Gamma \\
\boldsymbol{q}=\int_{\Gamma_{f i}} \tilde{\boldsymbol{U}}^{T} \boldsymbol{t}_{j} d \Gamma \\
\boldsymbol{e}=\int_{\Gamma_{f i}} \boldsymbol{T}_{c}^{\boldsymbol{T}} \boldsymbol{u}_{j} d \Gamma
\end{gathered}
$$

para a totalidade de problemas globais.

A partir das considerações anteriores, para cada $\mathbf{P G}_{\mathbf{k}}$ a aproximação do campo de tensões nos elementos é ditada pela Eq. (3.1), sendo representada por:

$$
\boldsymbol{\sigma}_{G \boldsymbol{k}}=\boldsymbol{S}_{c} \mathbf{c}, \mathrm{em} \Omega_{\mathrm{e}}
$$

Por sua vez a aproximação básica do campo de deslocamentos na fronteira estática dos elementos, para cada $\mathbf{P G}_{\mathbf{k}}$, é dada pela Eq. (3.5), admitindo o aspecto:

$$
\tilde{\mathbf{u}}_{G \boldsymbol{k}}=\tilde{\mathbf{U} d} ; \mathrm{em} \Gamma_{\mathrm{te}}
$$

Em particular, numa fronteira enriquecida, deve-se substituir a aproximação básica pela aproximação ditada pela Eq. (4.4), ou seja:

$$
\tilde{\mathbf{u}}_{G \boldsymbol{k}}=\tilde{\mathbf{U}}_{E} \mathbf{d}_{\mathrm{E}} ; \text { em } \Gamma_{\mathrm{E}} \subset \Gamma_{\mathrm{te}}
$$

Finalmente, a análise se completa com aproximação da distribuição de tensões nas linhas $\mathbf{L}_{\mathrm{fi}}$ correspondentes às fissuras previamente desconsideradas. Para uma fissura $f_{i}$ a 
distribuição de tensões pode ser obtida de maneira análoga a realizada no problema global $\mathbf{P G}_{0}$ (vide Eq. (5.15)). Assim sendo, para cada problema global $\mathbf{P G}_{\mathbf{k}}$ escreve-se:

$$
\boldsymbol{Q}_{G k}=\boldsymbol{R}_{\alpha} \boldsymbol{t}, \text { em } \Gamma_{\text {te }} \cap \mathbf{L}_{\mathrm{fi}}
$$

para $k=1, \cdots, n_{p}$.

Por conseguinte, a distribuição de tensões resultantes da sobreposição dos $n_{p}$ problemas globais na linha de uma fissura $f_{i}$ fica com o aspecto:

$$
\boldsymbol{t}_{G}=\sum_{k=1}^{n p} \alpha_{k} \boldsymbol{Q}_{G k}=\boldsymbol{Q}_{G} \boldsymbol{\alpha}, \mathrm{em} \mathbf{L}_{\mathrm{fi}}
$$

onde as grandezas envolvidas já foram apresentadas no item 5.2.3.

\subsubsection{Determinação dos fatores de escala}

Considerando-se a totalidade de problemas locais e globais correspondentes a todas as fissuras do PVC, os fatores de escala das Eqs. (5.10) e (5.11) são determinados a partir do sistema formado pelo uso sistemático da Eq. (5.34), considerando-se todas as fissuras do problema:

$$
K_{p i} \alpha=-v_{p i}
$$

onde $\boldsymbol{K}_{\boldsymbol{p} \boldsymbol{i}}=\int_{L_{f i}} \boldsymbol{Q}_{L}^{T}\left(\boldsymbol{Q}_{G}-\boldsymbol{Q}_{\boldsymbol{L}}\right) d L$, para $i=1, \ldots, n$, sendo $n$ o número de fissuras (vide as observações acerca da Eq. (5.9)).

\subsection{Fatores de intensidade de tensão}

Neste trabalho, na extração dos fatores de intensidade de tensão utiliza-se a técnica de correlação dos deslocamentos, a qual foi empregada com sucesso em análises realizadas por Bittencourt et. al (2003), Leonel (2006), entre outros. Tal técnica caracteriza-se pela correlação entre os valores analíticos e aproximados de deslocamentos obtidos na região próxima à extremidade da fissura.

Segundo Westergard ${ }^{1}(1939$ apud LEONEL, 2006) as componentes do campo de deslocamentos no entorno da extremidade de uma fissura podem ser determinadas mediante as Eqs. (5.35) e (5.36). 


$$
\begin{aligned}
& u_{\eta}=\frac{K_{\mathrm{I}}}{2 G} \sqrt{\frac{r}{2 \pi}} \cos \left(\frac{\phi}{2}\right)\left[(\kappa-1)+2 \operatorname{sen}^{2}\left(\frac{\phi}{2}\right)\right]+\frac{K_{\mathrm{II}}}{2 G} \sqrt{\frac{r}{2 \pi}} \operatorname{sen}\left(\frac{\phi}{2}\right)\left[(\kappa+1)+2 \cos ^{2}\left(\frac{\phi}{2}\right)\right] \\
& u_{\zeta}=\frac{K_{\mathrm{I}}}{2 G} \sqrt{\frac{r}{2 \pi}} \operatorname{sen}\left(\frac{\phi}{2}\right)\left[(\kappa+1)-2 \cos ^{2}\left(\frac{\phi}{2}\right)\right]+\frac{K_{\mathrm{II}}}{2 G} \sqrt{\frac{r}{2 \pi}} \cos \left(\frac{\phi}{2}\right)\left[(\kappa-1)+2 \operatorname{sen}^{2}\left(\frac{\phi}{2}\right)\right]
\end{aligned}
$$

onde:

- $u_{\eta}$ e $u_{\zeta}$ são as componentes do campo de deslocamentos nas direções paralela e perpendicular às faces da fissura, respectivamente.

- $K_{\mathrm{I}}$ e $K_{\mathrm{II}}$ são os fatores de intensidade de tensão, respectivamente, para os modos de abertura e deslizamento.

- $G=\frac{E}{2(1+v)}$ é o modulo de elasticidade transversal.

- $\quad r$ é a distância de um ponto considerado à extremidade da fissura.

- $\phi$ é o ângulo do ponto considerado relativo à bissetriz da abertura da fissura.

- $\kappa=3-4 v$ para o EPD ou $\kappa=\frac{3-v}{1+v}$ para o EPT.

Os fatores de intensidade de tensão podem ser determinados considerando-se dois pontos contíguos próximos à extremidade da fissura e pertencentes às suas faces, conforme ilustrado na Figura (5.9).

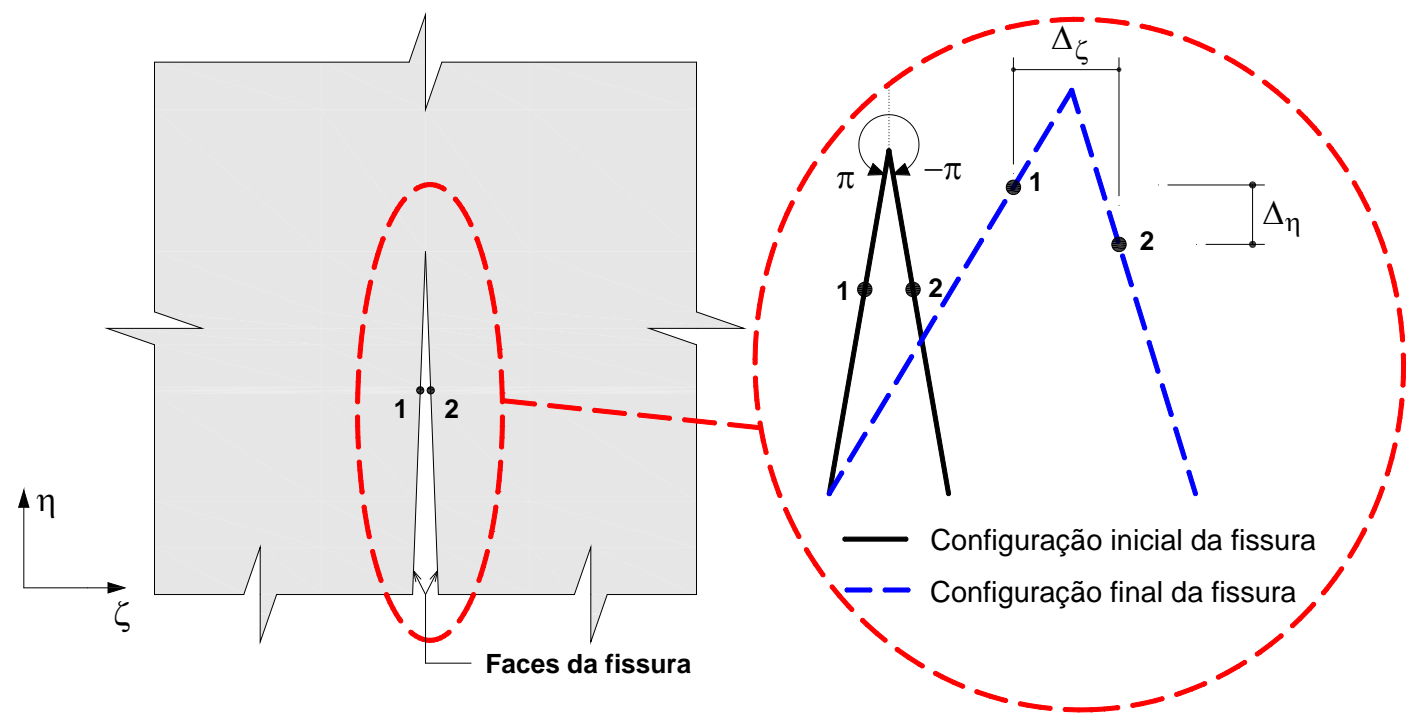

Figura 5.9. Pontos nodais considerados na avaliação dos fatores de intensidade de tensão.

As expressões obtidas da avaliação da Eq. (5.35) ou (5.36), para os ângulos rasos $\pi$ e $-\pi$, quando subtraídas fornecem, em cada situação, as expressões que definem os fatores de intensidade de tensão em função dos deslocamentos relativos $\Delta_{\zeta}$ e $\Delta \eta$, segundo as direções 
perpendicular e paralela às faces da fissura dos pontos considerados (vide Figura (5.9)):

$$
\begin{aligned}
& K_{\text {I }}=\frac{G}{\kappa+1} \sqrt{\frac{2 \pi}{r}} \Delta_{\zeta} \\
& K_{\text {II }}=\frac{G}{\kappa+1} \sqrt{\frac{2 \pi}{r}} \Delta_{\eta}
\end{aligned}
$$

A técnica de correlação dos deslocamentos se completa, mediante a avaliação numérica das Eqs. (5.37) e (5.38), aproximando-se os deslocamentos relativos nos pontos de interesse. Em particular, numa análise mediante elementos finitos consideram-se os pontos nodais sobre as faces da fissura mais próximos de sua extremidade, conforme ilustrado na Figura (5.10).

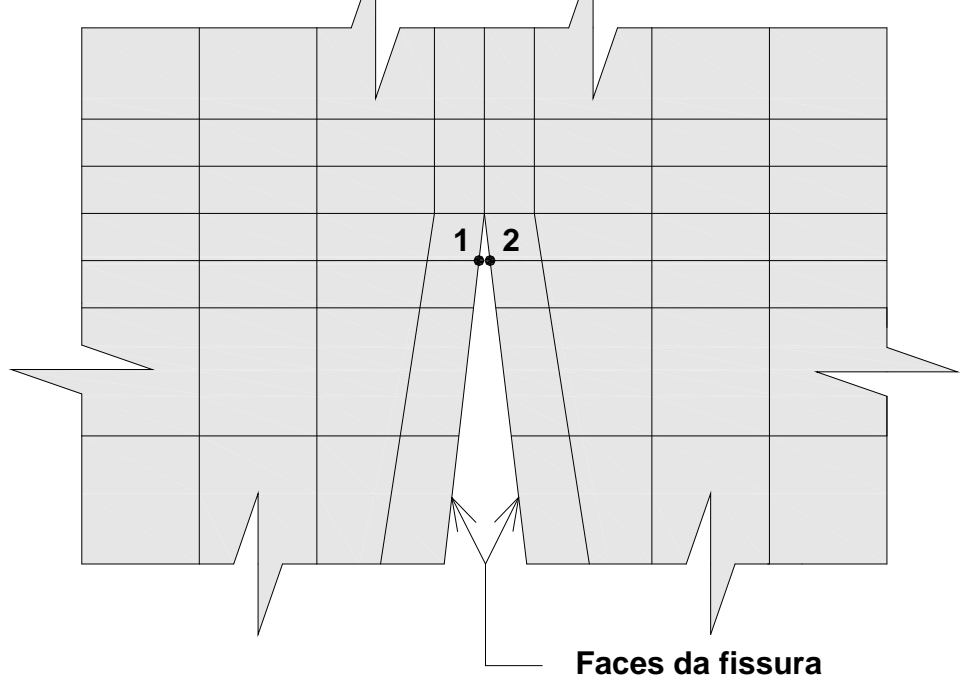

Figura 5.10. Pontos considerados numa análise via MEF para a extração dos fatores de intensidade de tensão mediante a técnica de correlação dos deslocamentos.

Na aproximação dos deslocamentos dos pontos indicados na Figura (5.10), quando da aplicação do Método da Partição em formulação híbrida-Trefftz com enriquecimento seletivo, utiliza-se a Eq. (5.10). 


\section{Implementação numérica}

\subsection{Considerações iniciais}

Neste capítulo apresentam-se as estratégias utilizadas na implementação numérica da formulação híbrida-Trefftz com enriquecimento seletivo. Primeiramente, são mencionados aspectos relevantes do código computacional empregado. Logo em seguida, descrevem-se os sistemas coordenados adotados na referida formulação. Num outro contexto, são descritos os recursos utilizados na implementação do elemento finito com domínio contínuo e do elemento com orifício circular, tais como: integração numérica e condicionamento numérico. Em seguida, abordam-se os aspectos relativos a todo o processo de implementação no tocante tanto à montagem e solução do sistema resolutivo quanto ao pós-processamento. Finalmente, mencionam-se as etapas inerentes à implementação do Método da Partição em formulação híbrida-Trefftz com enriquecimento seletivo.

\subsection{Código computacional}

O programa desenvolvido neste trabalho foi escrito em linguagem de programação FORTRAN. Optou-se pela programação modular, sendo concebidos os seguintes módulos:

- Variáveis globais: módulo constituído das variáveis comuns a todos os módulos do programa.

- Leitura de dados: utilizado na interface com o usuário e na leitura geral dos dados mediante arquivos de entrada.

- Pontos de Gauss: módulo que armazena pontos e pesos de Gauss utilizados na integração numérica. São disponibilizados de cinco até sessenta pontos de Gauss.

- Matrizes dos elementos: utilizado na geração de todas as matrizes e vetores dos elementos. 
- Enriquecimento: módulo que gera as matrizes e vetores provenientes do enriquecimento seletivo.

- Montagem: utilizado na montagem do sistema global.

- Resolução: módulo que impõe as condições de contorno dos nós da discretização adotada e resolve o sistema global.

- Armazena valores: utilizado na armazenagem dos valores de interesse para a impressão dos dados.

- Partição: módulo que utiliza os módulos anteriores em cada uma das etapas de execução do Método da Partição em formulação híbrida-Trefftz com enriquecimento seletivo, além da leitura de outros arquivos de entrada de dados referentes às características dos problemas locais e globais.

- Impressão: utilizado na impressão de todos os dados de interesse e para saída gráfica de tensões.

Visando-se minimizar o tempo de processamento em todo o processo de implementação, tomaram-se cuidados relativos ao uso de memória, mediante alocação dinâmica.

\subsection{Sistemas de coordenadas empregados na implementação}

$\mathrm{Na}$ implementação da formulação híbrida-Trefftz com enriquecimento seletivo utilizam-se três sistemas de interesse: o global, o local no domínio dos elementos e o adimensional nas fronteiras estáticas. Tais sistemas devem, evidentemente, ser interrelacionados na concepção da formulação estudada.

O sistema global é utilizado na interface com o usuário mediante a entrada de dados. Nesse sentido, adota-se um sistema de coordenadas cartesianas de origem arbitrária para a identificação de cada ponto da discretização adotada.

Por sua vez, o sistema local no domínio dos elementos é utilizado em virtude das características da formulação, a qual se baseia na discretização do domínio num conjunto de elementos finitos para construção da aproximação do campo de tensões. Conforme já mencionado, neste trabalho, tanto nos elementos finitos com domínios contínuos quanto nos elementos com orifício circular, adotou-se o centróide do elemento como origem local deste 
sistema.

Finalmente, no contorno de cada elemento da discretização adotada estabelece-se um sistema local de coordenadas adimensionais, tanto para a construção da aproximação do campo de deslocamentos quanto para ser utilizado nas integrações numéricas. Neste caso, como já exposto, para cada lado do elemento adota-se o respectivo ponto médio como origem do sistema adimensional de coordenadas locais. Além disso, para compatibilizar os sistemas destas coordenadas, quando se considera uma rede de elementos, adota-se, no contorno de cada elemento, o 'fluxo' indicado na Figura (6.1), partindo-se do ponto mais inferior à esquerda até o ponto mais superior à direita.

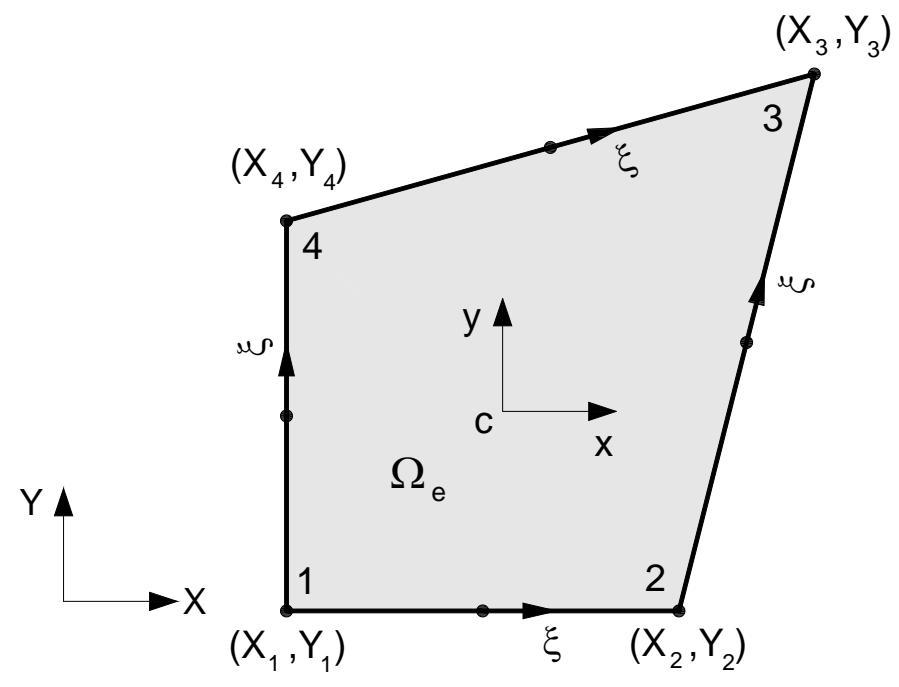

Figura 6.1. Sistemas de coordenadas no elemento finito com domínio contínuo.

\subsection{Implementação do elemento finito com domínio contínuo}

\subsubsection{Posição do centróide}

Considere-se o elemento indicado na Figura (6.1) onde estão indicados todos os sistemas de interesse. A partir das coordenadas nodais, as quais são inicialmente referidas ao sistema global, é possível determinar a posição do centróide do elemento mediante o teorema de Green. Tal teorema permite que se calculem integrais em domínios bidimensionais através de integrais curvilíneas definidas sobre o contorno do objeto analisado. Em particular, para o elemento finito indicado na Figura (6.1) as coordenadas $\bar{X}$ e $\bar{Y}$ do centróide $c$ podem ser obtidas das relações dadas, respectivamente, pelas Eqs. (6.1) e (6.2) (SWOKOWSKI, 1983). 


$$
\begin{aligned}
& \bar{X}=\frac{1}{2 A} \oint_{\Gamma_{e}} X^{2} d Y \\
& \bar{Y}=-\frac{1}{2 A} \oint_{\Gamma_{e}} Y^{2} d X
\end{aligned}
$$

sendo $A=\oint_{\Gamma_{e}} X d Y$ a área do elemento

Substituindo-se as expressões analíticas de cada segmento de reta em $\Gamma_{\mathrm{e}}$ nas Eqs. (6.1) e (6.2) e efetuando-se as operações necessárias resulta:

$$
\begin{aligned}
\bar{X}= & {\left[\left(X_{1}^{2}+X_{1} X_{2}+X_{2}{ }^{2}\right)\left(-Y_{1}+Y_{2}\right)+\left(X_{2}{ }^{2}+X_{2} X_{3}+X_{3}{ }^{2}\right)\left(-Y_{2}+Y_{3}\right)+\right.} \\
& \left.\left(X_{1}^{2}+X_{1} X_{4}+X_{4}{ }^{2}\right)\left(Y_{1}-Y_{4}\right)+\left(X_{3}{ }^{2}+X_{3} X_{4}+X_{4}{ }^{2}\right)\left(-Y_{3}+Y_{4}\right)\right] / 6 A \\
\bar{Y}= & \left\{-X_{2}\left(Y_{1}-Y_{3}\right)\left(Y_{1}+Y_{2}+Y_{3}\right)+X_{4}\left(Y_{1}-Y_{3}\right)\left(Y_{1}+Y_{3}+Y_{4}\right)+\right. \\
& \left.\left(Y_{2}-Y_{4}\right)\left[X_{1}\left(Y_{1}+Y_{2}+Y_{4}\right)-X_{3}\left(Y_{2}+Y_{3}+Y_{4}\right)\right]\right\} / 6 \mathrm{~A}
\end{aligned}
$$

onde a área do elemento é dada por:

$$
A=\frac{1}{2}\left[-\left(X_{2}-X_{4}\right)\left(Y_{1}-Y_{3}\right)+\left(X_{1}-X_{3}\right)\left(Y_{2}-Y_{4}\right)\right]
$$

\subsubsection{Mapeamento geométrico e determinação dos co-senos diretores}

Uma vez determinadas as coordenadas do centróide do elemento pode-se estabelecer o sistema local de coordenadas no domínio do elemento. Neste sistema, as coordenadas nodais do elemento adquirem o aspecto:

$$
\begin{aligned}
& x_{k}=X_{k}-\bar{X}, \text { para } k=1, \cdots, 4 \\
& y_{k}=Y_{k}-\bar{Y}, \text { para } k=1, \cdots, 4
\end{aligned}
$$

onde $x_{k}$ e $y_{k}, k=1, \cdots, 4$, são as coordenadas cartesianas de cada nó do elemento segundo o sistema local de coordenadas.

Conforme já apresentado, as integrações de todas as matrizes e vetores integrantes do sistema resolvente da formulação híbrida-Trefftz com enriquecimento seletivo restringem-se ao contorno do elemento. Assim sendo, deve-se expressar as coordenadas $x$ e $y$ no domínio do elemento em função da coordenada adimensional $\xi \in[-1,1]$ definida no contorno $\Gamma_{\mathrm{e}}$, antes de proceder-se com as integrações numéricas. Este mapeamento depende das coordenadas nodais iniciais e finais pertencentes a cada lado considerado, conforme ilustrado na Figura (6.2). 


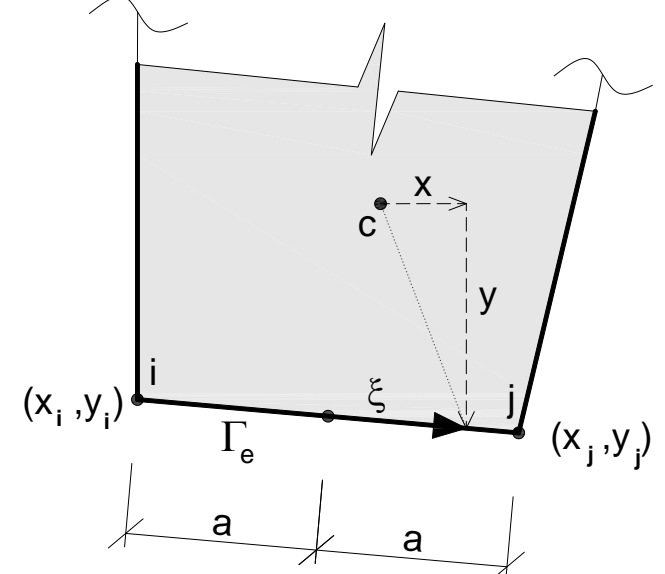

Figura 6.2. Mapeamento de um dos lados do elemento.

Sendo as distribuições em $x$ e $y$ lineares e denotando-se por $i$ e $j$ os nós inicial e final do lado analisado obtêm-se:

$$
\begin{aligned}
& x=\frac{x_{j}-x_{i}}{2} \xi+\frac{x_{j}+x_{i}}{2} \\
& y=\frac{y_{j}-y_{i}}{2} \xi+\frac{y_{j}+y_{i}}{2}
\end{aligned}
$$

onde $x_{i}, x_{j}, y_{i}$ e $y_{j}$ são as coordenadas cartesianas dos referidos nós segundo o sistema local de coordenadas cartesianas no domínio do elemento (vide Figura (6.2)).

A partir das coordenadas dadas pelas Eqs. (6.8) e (6.9), os co-senos diretores de um determinado lado podem ser obtidos pelo produto vetorial entre dois vetores unitários. Um na direção paralela ao lado e o outro perpendicular ao plano do elemento. Assim sendo, escrevese:

$$
\boldsymbol{n}=\frac{\left\{x_{j}-x_{i}, y_{j}-y_{i}, 0\right\}}{\sqrt{\left(x_{j}-x_{i}\right)^{2}+\left(y_{j}-y_{i}\right)^{2}}} \times\{0,0,1\}
$$

onde $\boldsymbol{n}^{T}=\left\{n_{x}, n_{y}, n_{z}\right\}$ é o vetor que coleta os co-senos diretores de um determinado lado segundo as direções $x, y$ e $z$, respectivamente.

Efetuando-se o produto vetorial indicado na Eq. (6.10) resulta:

$$
\begin{aligned}
& n_{x}=\frac{y_{j}-y_{i}}{\sqrt{\left(x_{j}-x_{i}\right)^{2}+\left(y_{j}-y_{i}\right)^{2}}} \\
& n_{y}=-\frac{x_{j}-x_{i}}{\sqrt{\left(x_{j}-x_{i}\right)^{2}+\left(y_{j}-y_{i}\right)^{2}}} \\
& n_{z}=0
\end{aligned}
$$




\subsubsection{Condicionamento numérico do sistema resolvente}

Com o intuito de garantir boas condições numéricas do sistema resolutivo, as coordenadas $x$ e $y$ dada pelas Eqs. (6.8) e (6.9) do sistema local no domínio do elemento foram tornadas adimensionais. Neste sentido, considerou-se uma distância de normalização $d_{N}$ dada pela média aritmética das distâncias de cada nó do elemento ao seu centróide, ou seja:

$$
d_{N}=\frac{1}{4} \sum_{k=1}^{4} \sqrt{\left(x_{k}^{2}+y_{k}^{2}\right)}
$$

Então, as coordenadas adimensionais admitem o aspecto:

$$
\begin{aligned}
& x_{N}=x / d_{N} \\
& y_{N}=y / d_{N}
\end{aligned}
$$

Assim sendo, considera-se que no processo de implementação, a matriz $\boldsymbol{N}_{c}$ seja referida ao sistema adimensional de coordenadas no domínio do elemento, mediante a substituição das coordenadas cartesianas $x$ e $y$ pelas coordenadas adimensionais $x_{N}$ e $y_{N}$, respectivamente.

Em virtude da mudança de configuração da matriz $\boldsymbol{N}_{c}$ verifica-se que a matriz $\boldsymbol{S}_{c}$, referida ao sistema adimensional, deve ser dividida pela distância de normalização $d_{N}$. De fato, sendo o operador $L_{c}$ aplicado sobre as coordenas cartesianas originais $x$ e $y$ da matriz $\boldsymbol{N}_{\boldsymbol{c}}$ (vide Eq. (3.1)), a partir da regra da cadeia escreve-se:

$$
\boldsymbol{L}_{\boldsymbol{c}}=\left[\begin{array}{ccc}
\partial / \partial \mathbf{x} & 0 & \partial / \partial \mathrm{y} \\
0 & \partial / \partial \mathbf{y} & \partial / \partial \mathbf{x}
\end{array}\right]=\left[\begin{array}{cccc}
\frac{\partial}{\partial x_{N}} \cdot \frac{\partial x_{N}}{\partial x} & 0 & \frac{\partial}{\partial y_{N}} \cdot \frac{\partial y_{N}}{\partial y} \\
0 & \frac{\partial}{\partial y_{N}} \cdot \frac{\partial y_{N}}{\partial y} & \frac{\partial}{\partial x_{N}} \cdot \frac{\partial x_{N}}{\partial x}
\end{array}\right]
$$

Considerando-se as Eqs. (6.15), (6.16) e (6.17), resulta:

$$
L_{c}=\frac{1}{d_{N}} \bar{L}_{c}
$$

sendo $\bar{L}_{c}=\left[\begin{array}{ccc}\partial / \partial x_{N} & 0 & \partial / \partial y_{N} \\ 0 & \partial / \partial y_{N} & \partial / \partial x_{N}\end{array}\right]$.

Finalmente, a partir da definição da matriz $\boldsymbol{S}_{c}$, contata-se:

$$
S_{c}=\frac{1}{d_{N}} D \bar{L}_{c}^{T} N_{c}
$$

onde todas as grandezas envolvidas estão especificadas para o sistema local adimensional no 
domínio do elemento.

Por conseguinte, no processo de implementação, evidenciam-se alteração em todas as matrizes e vetores dependentes da matriz $\boldsymbol{S}_{\boldsymbol{c}}$ referida ao sistema local adimensional no elemento, quais sejam:

$$
\begin{gathered}
\boldsymbol{F}=\frac{1}{d_{N}} \int_{\Gamma_{e}} T_{c}{ }^{T} \boldsymbol{N}_{c} d \Gamma \\
\boldsymbol{G}=\frac{1}{d_{N}} \int_{\Gamma_{t e}} \boldsymbol{T}_{c}{ }^{T} \tilde{\boldsymbol{U}} d \Gamma \\
\boldsymbol{e}=\frac{1}{d_{N}} \int_{\Gamma_{u e}} \boldsymbol{T}_{c}{ }^{\boldsymbol{T}} \overline{\boldsymbol{u}}_{c} d \Gamma \\
\boldsymbol{G}=\frac{1}{d_{N}} \int_{\Gamma_{t e}} \boldsymbol{T}_{c}^{T} \tilde{\boldsymbol{U}} d \Gamma
\end{gathered}
$$

onde, $\boldsymbol{T}_{c}=\boldsymbol{A} \overline{\boldsymbol{S}}_{c}$, com $\overline{\boldsymbol{S}}_{c}$ apresentando a forma: $\overline{\boldsymbol{S}}_{\boldsymbol{c}}=\boldsymbol{D} \overline{\boldsymbol{L}}_{c}{ }^{T} \boldsymbol{N}_{c}$ com as variáveis normalizadas.

\subsubsection{Integração numérica}

$\mathrm{Na}$ integração numérica utiliza-se a consagrada técnica da quadratura de GaussLegendre. Tal técnica caracteriza-se pela substituição de uma integral definida por um somatório de termos resultantes do produto entre valores funcionais e pesos adequados estabelecidos no intervalo de integração, ou seja:

$$
\int_{a}^{b} f(x) d x=w_{1} f\left(x_{1}\right)+\cdots+w_{n} f\left(x_{n}\right), \text { com } x_{i} \in[a, b] \text { para } i=1, n
$$

onde $f\left(x_{i}\right)$ são valores funcionais e $w_{i}$ os respectivos pesos, para $i=1, n$.

Segundo a referida técnica, a partir de $n$ pontos tomados no intervalo de integração é possível calcular a integral exata de um polinômio de até grau $2 n-1$. Neste trabalho utilizouse esta condição em todas as integrações polinomiais.

Para aplicação da Eq. (6.24) na integração de todas as matrizes e vetores do sistema resolvente considerou-se a variável de integração adimensional $\xi$ definida no intervalo de $[-1,1]$ sobre cada um dos lados do elemento. Desta forma, o processo de integração realiza-se trecho a trecho, tornando-se necessário expressar tanto as coordenadas cartesianas adimensionais $x_{N}$ e $y_{N}$ em função da coordenada $\xi$, quanto a diferencial $\mathrm{d} \Gamma$ em função da diferencial $d \xi$. 
Assim sendo, a partir da combinação das Eqs. (6.8) e (6.9) com as Eqs. (6.15) e (6.16), resulta:

$$
\begin{aligned}
& x_{N}=\frac{1}{d_{N}}\left(\frac{x_{j}-x_{i}}{2} \xi+\frac{x_{j}+x_{i}}{2}\right) \\
& y_{N}=\frac{1}{d_{N}}\left(\frac{y_{j}-y_{i}}{2} \xi+\frac{y_{j}+y_{i}}{2}\right)
\end{aligned}
$$

enquanto do cálculo diferencial obtém-se a relação de interesse entre as referidas diferenciais:

$$
d \Gamma=\frac{1}{2} \sqrt{\left(x_{j}-x_{i}\right)^{2}+\left(y_{j}-y_{i}\right)^{2}} d \xi
$$

onde, conforme já mencionado, $x_{i}, x_{j}, y_{i}$ e $y_{j}$ referem-se às coordenadas cartesianas de dois nós consecutivos de um determinado lado do elemento segundo o sistema local.

\subsection{Implementação do elemento finito com orifício circular central}

Conforme já apresentado, no elemento finito retangular com orifício circular considera-se um sistema local em coordenadas polares na construção das aproximações dos campos de tensões e deslocamentos em seu domínio. Consequentemente, evidenciam-se algumas alterações no processo de implementação deste elemento quando comparado àquele do elemento com domínio contínuo.

Assim sendo, considere-se o elemento indicado na Figura (6.3), onde se representam todos os sistemas de interesse.

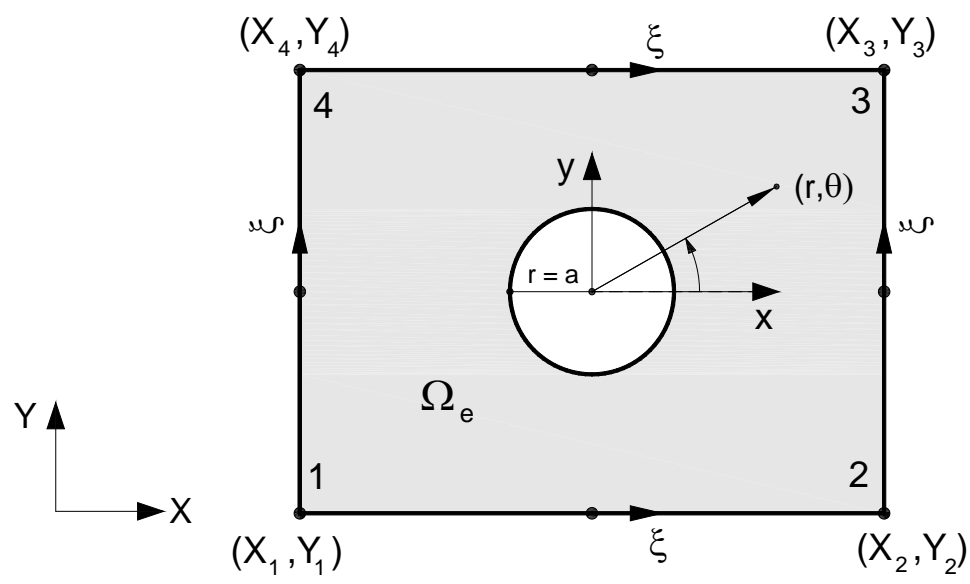

Figura 6.3. Sistemas de coordenadas no elemento finito com orifício.

Analisando-se o elemento indicado na Figura (6.3) determina-se sem maiores 
dificuldades a posição de seu centróide $c$ mediante as seguintes equações:

$$
\begin{aligned}
& \bar{X}=\left(X_{1}+X_{2}+X_{3}+X_{4}\right) / 4 \\
& \bar{Y}=\left(Y_{1}+Y_{2}+Y_{3}+Y_{4}\right) / 4
\end{aligned}
$$

A partir da posição do centróide, verifica-se que as coordenadas nodais do elemento $x_{k}$ e $y_{k}$, para $k=1, \cdots, 4$, segundo o sistema cartesiano local continuam sendo ditadas pelas Eqs. (6.6) e (6.7), enquanto, os co-senos diretores ainda são obtidos pelas Eqs. (6.11), (6.12) e (6.13), os quais foram obtidos a partir de condições mais gerais de geometria.

No elemento indicado na Figura (6.3), o mapeamento em $\Gamma_{\mathrm{e}}$ realiza-se mediante uma relação entre cada uma das coordenadas polares $r$ e $\theta$ com a coordenada adimensional $\xi$. Neste contexto quatro situações são possíveis, a depender do lado considerado.

Desta forma, considere-se o mapeamento do lado inferior do elemento, conforme ilustrado na Figura (6.4).

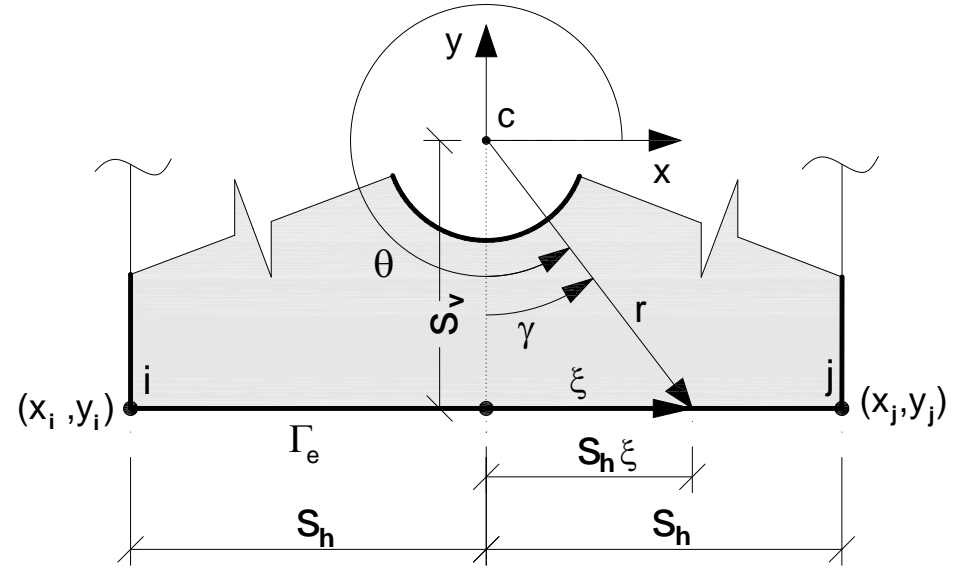

Figura 6.4. Mapeamento de um dos lados do elemento com orifício.

Nesta situação a coordenada $r$ admite o aspecto:

$$
r=\sqrt{s_{v}{ }^{2}+s_{h}{ }^{2} \xi^{2}}
$$

sendo $s_{h}$ e $s_{v}$ a metade do comprimento dos lados horizontal e vertical, respectivamente (vide figura (6.4)), enquanto a coordenada $\theta$ pode ser representada por:

$$
\theta=\frac{3 \pi}{2}+\gamma
$$

sendo $\gamma=\operatorname{arcsen}\left[s_{h} \xi / \sqrt{s_{v}{ }^{2}+s_{h}{ }^{2} \xi^{2}}\right]$.

Analogamente, seguindo-se este procedimento para as situações restantes, obtêm-se:

$$
r=\sqrt{s_{h}{ }^{2}+s_{v}{ }^{2} \xi^{2}}
$$




$$
\theta=\operatorname{arcsen}\left[s_{v} \xi / \sqrt{s_{h}{ }^{2}+s_{v}{ }^{2} \xi^{2}}\right]
$$

para o lado direito.

$$
\begin{aligned}
& r=\sqrt{s_{v}{ }^{2}+s_{h}{ }^{2} \xi^{2}} \\
& \theta=\frac{\pi}{2}-\operatorname{arcsen}\left[s_{h} \xi / \sqrt{s_{v}{ }^{2}+s_{h}{ }^{2} \xi^{2}}\right]
\end{aligned}
$$

para o lado superior, e:

$$
\begin{aligned}
& r=\sqrt{\mathrm{s}_{\mathrm{h}}{ }^{2}+\mathrm{s}_{\mathrm{v}}{ }^{2} \xi^{2}} \\
& \theta=\pi-\operatorname{arcsen}\left[\mathrm{s}_{\mathrm{v}} \xi / \sqrt{\mathrm{s}_{\mathrm{h}}{ }^{2}+\mathrm{s}_{\mathrm{v}}{ }^{2} \xi^{2}}\right]
\end{aligned}
$$

para o lado esquerdo.

De maneira similar a realizada para o elemento com domínio contínuo, para garantir boas condições numéricas do sistema resolvente, utilizam-se coordenadas adimensionais no processo de implementação. Neste caso, apenas a variável $r$ deve ser normalizada, uma vez que a variável $\theta$ já está expressa em radianos.

Assim sendo, considerando-se que a distância de normalização seja dada pela Eq. (6.14), obtém-se a coordenada adimensional de interesse:

$$
r_{N}=r / d_{N}
$$

Seguindo-se os mesmos procedimentos executados no processo de normalização das variáveis $x$ e $y$ do sistema local, quando de um elemento com domínio contínuo, para a variável $r$ de um elemento com orifício circular, evidenciam-se as seguintes alterações nas matrizes e vetores do sistema resolvente:

$$
\begin{gathered}
\boldsymbol{F}=\frac{1}{d_{N}} \int_{\Gamma_{e}} \boldsymbol{T}_{\boldsymbol{p}}{ }^{\boldsymbol{T}} \boldsymbol{R}_{u \theta} \boldsymbol{N}_{\boldsymbol{p}} d \Gamma \\
\boldsymbol{G}=\frac{1}{d_{N}} \int_{\Gamma_{t e}} \boldsymbol{T}_{p}^{\boldsymbol{T}} \tilde{\boldsymbol{U}} d \Gamma \\
\boldsymbol{e}=\frac{1}{d_{N}} \int_{\Gamma_{u e}} \boldsymbol{T}_{p}^{T} \overline{\boldsymbol{u}}_{c} d \Gamma \\
\boldsymbol{G}=\frac{1}{d_{N}} \int_{\Gamma_{t e}} \boldsymbol{T}_{p}^{T} \tilde{\boldsymbol{U}} d \Gamma
\end{gathered}
$$

sendo $\boldsymbol{T}_{p}=\boldsymbol{A} \boldsymbol{R}_{\sigma \theta} \overline{\boldsymbol{S}}_{p}$, com $\overline{\boldsymbol{S}}_{\boldsymbol{p}}=\boldsymbol{D} \boldsymbol{M}_{\boldsymbol{\varepsilon}} \boldsymbol{N}_{\boldsymbol{p}}+\boldsymbol{D} \overline{\boldsymbol{L}}_{\boldsymbol{p}}{ }^{T} \boldsymbol{N}_{\boldsymbol{p}}$ definida a partir das variáveis adimensionais, de maneira similar ao que foi realizado para o sistema coordenado.

Nas Eqs. (6.39)-(6.42) as matrizes $\boldsymbol{N}_{p}$ e $\overline{\boldsymbol{S}}_{\boldsymbol{p}}$ são funções das coordenadas 
adimensionais $r_{N}$ e $\theta$. Para $\theta$, utilizam-se as expressões (6.31), (6.33), (6.35) e (6.37), enquanto para $r_{N}$, combina-se a Eq. (6.38) com cada uma das seguintes equações: Eqs. (6.30), (6.32), (6.34) e (6.36). Além disso, a relação entre as diferenciais $\mathrm{d} \Gamma \mathrm{e} \mathrm{d} \xi$ continua sendo ditada pela Eq. (6.27), a qual foi deduzida para uma situação mais geral de geometria.

Finalmente, na integração numérica, assim como nos elementos com domínios contínuos, utiliza-se a técnica de quadratura de Gauss-Legendre. Contudo, uma vez que as matrizes e vetores do sistema resolvente envolvem funções não polinomiais verifica-se que a integração numérica não é exata. Neste contexto, visando-se minimizar o erro decorrente deste processo, utilizou-se uma quantidade significativa de pontos de Gauss em cada um dos lados do elemento (sessenta pontos).

\subsection{Montagem do sistema resolvente global}

Com o intuito de facilitar o processo de implementação no tocante à montagem do sistema global ditado pela Eq. (4.22), considera-se que tanto a matriz dos coeficientes do sistema global quanto o vetor dos termos independentes sejam adequadamente decompostos em algumas parcelas de interesse.

Para a matriz dos coeficientes, denotada por $\boldsymbol{A}_{G}$, escreve-se:

$$
A_{G}=F_{G}+G_{G}+G_{G}^{T}+\Delta G_{G}+\Delta G_{G}^{T}
$$

sendo as matrizes $\boldsymbol{F}_{\boldsymbol{G}}, \boldsymbol{G}_{\boldsymbol{G}}$ e $\Delta \boldsymbol{G}_{\boldsymbol{G}}$ as matrizes resultantes da alocação das matrizes $\boldsymbol{F}, \boldsymbol{G}$ e $\Delta \boldsymbol{G}$ de cada elemento da discretização adotada, respectivamente.

Na construção de cada matriz $F_{G}, G_{G}$ e $\Delta G_{G}$ gera-se primeiramente uma matriz nula com a mesma dimensão de $\boldsymbol{A}_{G}$ e em seguida alocam-se adequadamente as matrizes de interesse de cada elemento da discretização adotada.

Já para o vetor de termos independentes, denotado por $\boldsymbol{B}_{\boldsymbol{G}}$, tem-se:

$$
\boldsymbol{B}_{G}=\boldsymbol{e}_{G}+\boldsymbol{q}_{t G}+\Delta \boldsymbol{q}_{t G}
$$

sendo os vetores $\boldsymbol{e}_{G}, \boldsymbol{q}_{t G}$ e $\Delta \boldsymbol{q}_{t G}$ os vetores resultantes da alocação dos vetores $\boldsymbol{e}, \boldsymbol{q}$ e $\Delta \boldsymbol{q}$ de cada elemento da discretização adotada.

De maneira análoga à realizada para as matrizes $F_{G}, G_{G}$ e $\Delta G_{G}$, na construção dos vetores $\boldsymbol{e}_{G}, \boldsymbol{q}_{t G}$ e $\Delta \boldsymbol{q}_{t G}$ concebe-se um vetor nulo com a mesma dimensão de $\boldsymbol{B}_{\boldsymbol{G}}$ e procedese com a alocação adequada dos vetores de interesse dos elementos da discretização adotada. 


\subsection{Solução do sistema resolvente}

Na solução do sistema resolvente foi utilizada a rotina interna DLSRG do FORTRAN. Tal rotina caracteriza-se pela resolução de sistemas lineares sem refinamento iterativo.

\subsection{Pós-processamento}

Conforme já apresentado, a solução do sistema resolvente ditado pela Eq. (4.22) é composta dos vetores $\boldsymbol{c}_{\boldsymbol{j}}$, que coleta os graus de liberdade em tensão no domínio de cada elemento, dos graus de liberdade nodais $\boldsymbol{d}_{\boldsymbol{t}}$ e dos graus de liberdade acrescidos em virtude do enriquecimento $\Delta \boldsymbol{d}_{\boldsymbol{t}}$. Além destas grandezas, são calculadas mediante o pós-processamento as seguintes:

\section{- Norma do resíduo do erro do sistema}

O sistema resolutivo global ditado pela Eq. (4.22) é aqui representado por:

$$
\boldsymbol{A}_{G} \boldsymbol{X}_{G}=\boldsymbol{B}_{G}
$$

onde $\boldsymbol{X}_{G}=\left\{\begin{array}{c}\boldsymbol{c}_{G} \\ \boldsymbol{d}_{\boldsymbol{t}} \\ \Delta \boldsymbol{d}_{\boldsymbol{t}}\end{array}\right\}$, sendo $\boldsymbol{c}_{G}=\left\{\begin{array}{c}\boldsymbol{c}_{\boldsymbol{1}} \\ \vdots \\ \boldsymbol{c}_{\boldsymbol{n}}\end{array}\right\}$ o vetor que coleta os vetores $\boldsymbol{c}_{j}$ dos $n$ elementos da discretização adotada.

Uma vez que a solução do sistema foi adotada sem refinamento iterativo, a partir da Eq. (6.45), o resíduo $\boldsymbol{R}_{\boldsymbol{G}}$ da solução admite o aspecto:

$$
\boldsymbol{R}_{G}=\boldsymbol{A}_{G} \boldsymbol{X}_{G}-\boldsymbol{B}_{G}
$$

enquanto sua norma, denotada por $\left\|R_{G}\right\|$, é obtida da relação:

$$
\left\|\boldsymbol{R}_{G}\right\|=\sqrt{\sum_{k=1}^{m}\left(R_{G}(k)\right)^{2}}
$$

sendo $R_{G}(k)$ as componentes do vetor $\boldsymbol{R}_{G}$ e $m$ sua dimensão. 


\section{- Energia de deformação}

Seja a energia de deformação $U$, definida por:

$$
U=\frac{1}{2} \sum \int_{\Omega_{e}} \varepsilon^{T} \sigma d \Omega
$$

onde $\boldsymbol{\varepsilon}=\boldsymbol{\varepsilon}_{c}$ e $\boldsymbol{\sigma}=\boldsymbol{\sigma}_{c}$, segundo o sistema cartesiano ou $\boldsymbol{\varepsilon}=\boldsymbol{\varepsilon}_{\boldsymbol{p}}$ e $\boldsymbol{\sigma}=\boldsymbol{\sigma}_{p}$, segundo o sistema polar.

Então, utilizando-se a relação constitutiva dada pela Eq. (2.5) ou (2.6) e inserindo-se a aproximação do campo de tensões no domínio do elemento, ditada pela Eq. (3.1) ou (3.32), resulta a aproximação:

$$
U=\frac{1}{2} \sum c^{T} F \boldsymbol{c}
$$

que é equivalente a

$$
U=\frac{1}{2} c_{G} \bar{F}_{G} c_{G}
$$

onde $\overline{\boldsymbol{F}}_{G}=\left(\begin{array}{ccc}\boldsymbol{F}_{1} & \cdots & \boldsymbol{0} \\ \vdots & \ddots & \vdots \\ \boldsymbol{0} & \cdots & \boldsymbol{F}_{\boldsymbol{n}}\end{array}\right)$ a matriz que coleta as matrizes $\boldsymbol{F}_{\boldsymbol{i}}$, para $i=1, \ldots, n$, sendo $n$ o número de elementos da discretização adotada.

\section{- Saída gráfica de tensões}

As tensões nos elementos são calculadas mediante a Eq. (3.1), quando de elementos com domínios contínuos ou pela Eq. (3.31), quando de elementos com orifício circular.

Nos elementos com domínios contínuos calculam-se as tensões nos nós e no centróide, enquanto nos elementos com orifício circular, além das tensões nodais, calculam-se as tensões sobre o contorno do orifício circular em oitos pontos regularmente dispostos.

Com o propósito de se obter uma maior regularidade na distribuição de tensões, considera-se que as tensões efetivas de um dado nó sejam obtidas pela média aritmética das tensões calculadas no referido nó, em cada um dos elementos que o compartilham.

A saída gráfica envolvendo as tensões nodais efetivas, as do centróide e as do contorno do orifício são realizadas com o software SURFER32 ${ }^{\circledR}$. 


\subsection{Implementação do Método da Partição}

$\mathrm{Na}$ implementação do Método da Partição em formulação híbrida-Trefftz com enriquecimento seletivo, como parte inicial do processo investigativo de pesquisa, considerou-se apenas a presença de uma única fissura no domínio estrutural, apesar da formulação apresentada no capítulo 6 contemplar domínios com múltiplas fissuras. Além disso, efetuaram-se outras simplificações, quais sejam:

- Nos problemas locais considerou-se o caminho $\Gamma_{f}$ como o próprio contorno do domínio local de influência $\boldsymbol{V}_{\boldsymbol{f}}$. Tal iniciativa é conveniente quando se adota um $\boldsymbol{V}_{\boldsymbol{f}}$ completamente impedido de se deslocar, uma vez que nesta situação apenas é necessário determinar a distribuição de tensões no contorno de $\boldsymbol{V}_{\boldsymbol{f}}$, ao invés de tensões e deslocamentos.

- Os carregamentos fictícios foram aplicados apenas na direção perpendicular à fissura, ao invés da aplicação nas direções perpendicular e paralela a ela.

- Em virtude da simplificação anterior só é plausível a extração do fator de intensidade de tensão relativo à abertura da fissura $\left(K_{\mathrm{I}}\right)$.

O processo de implementação da formulação geral fica como sugestão para desenvolvimentos futuros.

$\mathrm{Na}$ implementação executada neste trabalho aproveitou-se a maioria dos módulos concebidos inicialmente para a análise de problemas bidimensionais da Elasticidade via a formulação híbrida-Trefftz com enriquecimento seletivo. Estes módulos e outras rotinas específicas, relacionadas aos problemas locais e globais, foram concatenados num módulo específico denominado "Partição". Tal módulo constitui-se das seguintes etapas, descritas sucintamente:

- Análise do $\mathbf{P G}_{0}$ : onde se constrói o vetor $\boldsymbol{v}_{\boldsymbol{p}}$ do sistema ditado pela Eq. (5.34).

- Análise dos problemas locais $\mathbf{P L}_{\mathbf{k}}$ : nesta etapa armazenam-se as distribuições de tensões no contorno do domínio local $\boldsymbol{V}_{\boldsymbol{f}}$.

- Análise dos problemas globais $\mathbf{P L}_{\mathbf{k}}$ : onde se aplicam as tensões armazenadas no contorno de $\boldsymbol{V}_{\boldsymbol{f}}$ da etapa anterior e constrói-se a matriz $\boldsymbol{K}_{\boldsymbol{p}}$ do sistema ditado pela Eq. (5.34) 
- Solução do sistema $\boldsymbol{K}_{\boldsymbol{p}} \boldsymbol{\alpha}=\boldsymbol{v}_{\boldsymbol{p}}$ : nesta etapa obtêm-se os fatores de escala inerentes ao Método da Partição.

- Pós-processamento: onde se calcula o fator de intensidade de tensão $K_{\mathrm{I}}$ e promove-se a saída gráfica do campo de tensão pelo SURFER32 ${ }^{\circledR}$. 


\section{Análises numéricas}

\subsection{Considerações iniciais}

Neste capítulo, com o propósito de avaliar as potencialidades da formulação estudada neste trabalho, relatam-se as análises que foram realizadas em cinco problemas peculiares. Em tais análises averiguaram-se a convergência de algumas grandezas de interesse, tais como: energia de deformação, deslocamento nodal e fator de intensidade de tensão. Neste sentido, exploraram-se uma ou mais modalidades de refinamento, seja o refino-h, o refino-p ou o enriquecimento seletivo. Os valores aproximados foram confrontados com valores analíticos ou, no desconhecimento destes, com valores de referência, obtidos mediante o MEF convencional, com o uso do software ANSYS $^{\circledR}$.É importante destacar que na obtenção dos valores de referência via ANSYS $^{\circledR}$ considerou-se uma discretização a partir da qual novos refinamentos não produziram alterações significativas nas respostas.

Em todas as avaliações, utilizaram-se as bases apresentadas no capítulo 4, concebidas para a aproximação do campo de deslocamentos no contorno do elemento, as quais foram assim designadas:

- $\beta_{0}$ : Base original sem enriquecimento.

- $\beta_{1}$ : Base enriquecida com funções polinomiais hierárquicas.

- $\beta_{2}$ : Base enriquecida com funções polinomiais não hierárquicas.

- $\beta_{3}$ : Base enriquecida com funções não polinomiais envolvendo funções trigonométricas.

Neste contexto, ressalta-se que o teste do "mosaico" foi uma condição preestabelecida e imprescindível em todas as análises efetuadas.

Sem prejuízos para os objetivos deste trabalho, em todos os problemas admitiram-se um regime de pequenas deformações, materiais com comportamento elástico-linear e hipótese de forças volúmicas nulas. Além disso, por mera simplicidade, suprimiram-se as unidades de todas as grandezas envolvidas.

Por fim, ressalta-se que os comentários feitos acerca do dispêndio computacional, restringiram-se à sua associação com o número de graus de liberdade envolvido. 
Evidentemente este não é o único fator de influência. Uma análise mais acurada, envolvendo os tempos de processamento das operações efetuadas, não foi alvo de consideração rigorosa deste trabalho.

\section{2 $1^{\circ}$ Problema: chapa tracionada}

O primeiro problema refere-se a uma chapa submetida a certas condições de carregamento e vinculação, conforme ilustrado na Figura (7.1). No dimensionamento da chapa adotou-se $a=20$ e espessura unitária.

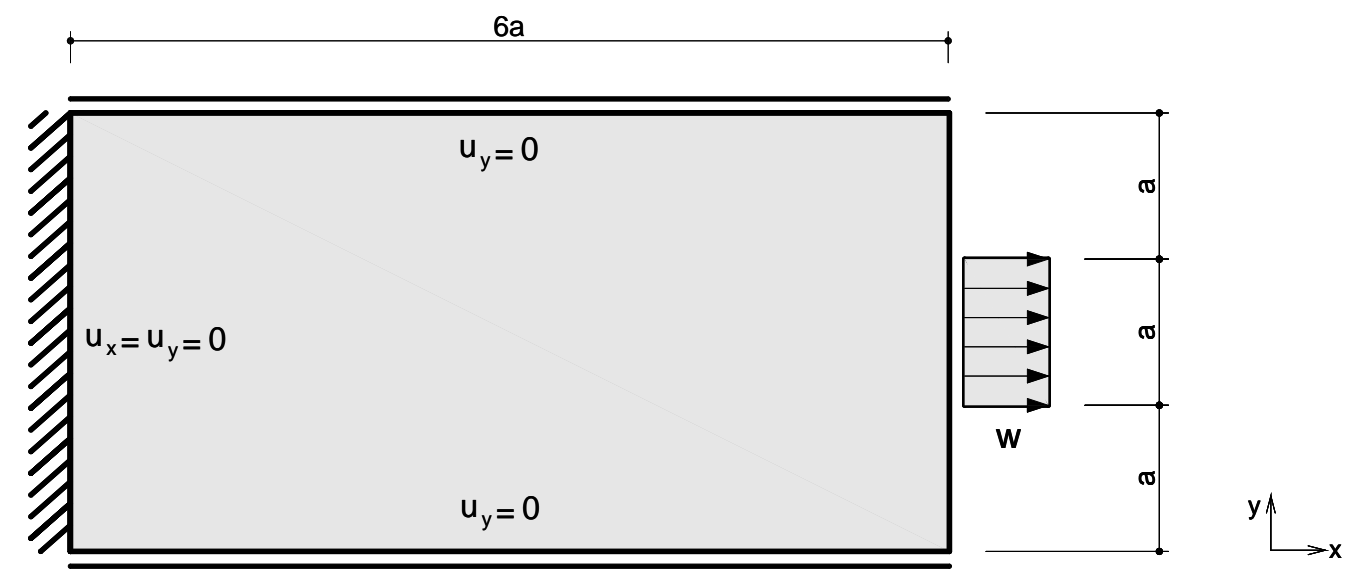

Figura 7.1. Chapa analisada no $1^{\circ}$ problema. Sua geometria, carregamento e vinculação.

A chapa encontra-se impedida de deslocar-se na aresta esquerda segundo as direções $x$ e $y$ e nas arestas inferior e superior somente segundo a direção $y$. Na aresta direita atua um carregamento uniformemente distribuído $W$ igual a 10. As propriedades do material, módulo de Young e o coeficiente de Poisson, apresentam, respectivamente, os valores: $E=1000$ e $v=0,3$.

Na obtenção dos valores de referência realizou-se uma análise via o MEF clássico com o auxílio do software ANSYS ${ }^{\circledR}$. A discretização que foi adotada nesta análise está indicada na Figura (7.2). Nela um total de 780 elementos triangulares foi distribuído segundo um refinamento prioritário no entorno da região de aplicação do carregamento. Nesta análise, utilizou-se o elemento triangular Plane 2, o qual possui seis nós e considera dois graus de liberdade por nó (deslocamentos em $x$ e $y$ ). Desta forma, 1628 nós foram envolvidos, o que totalizou 3256 graus de liberdade. 


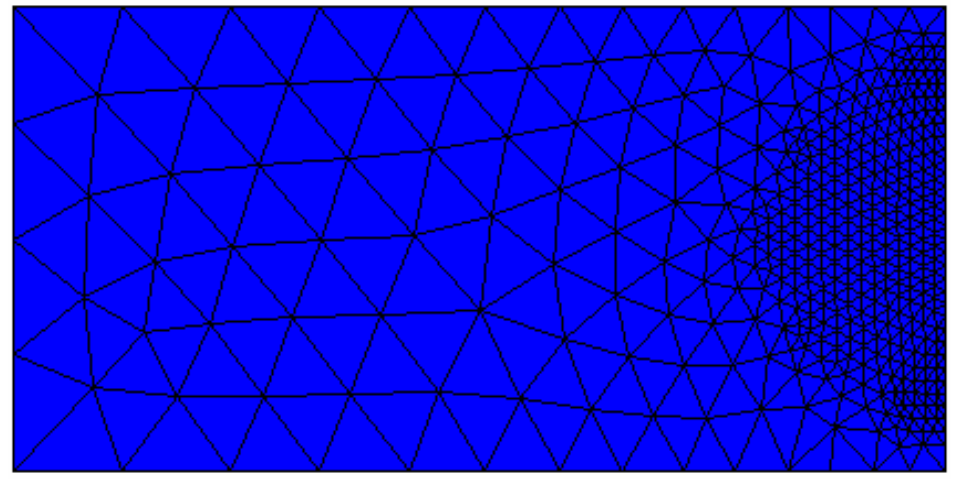

Figura 7.2. Discretização adotada na análise realizada via o MEF clássico para obtenção dos valores de referência do $1^{\circ}$ problema.

O valor de referência obtido para a energia de deformação $U_{R E F}$ foi de 46,47 . Para a obtenção das componentes de deslocamento considerou-se o canto superior direito, encontrando-se $u_{x \text { ref }}=0,2818$, segundo a direção horizontal e evidentemente $u_{y_{\text {ref }}}=0,0000$, segundo a direção vertical. Por sua vez, a distribuição de tensões obtida pode ser visualizada na Figura (7.3).

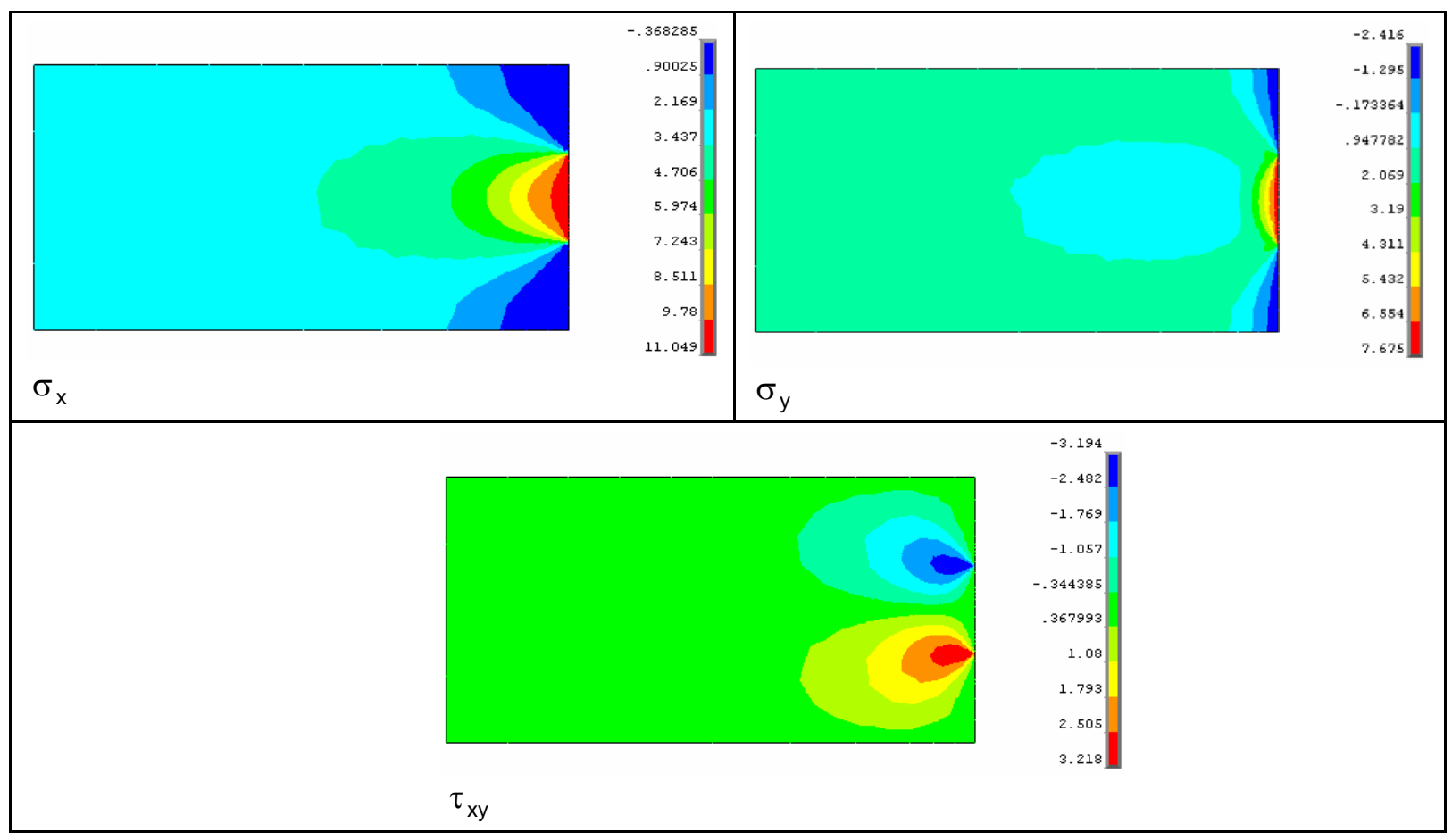

Figura 7.3. Distribuição de tensões de referência do $1^{\circ}$ problema.

Neste problema o desempenho da formulação estudada neste trabalho foi avaliado mediante o refino-h, o refino-p e o enriquecimento seletivo, conforme exposto a seguir. 


\section{- Refino-h}

No refino-h utilizou-se redes regulares de relação $2 k \times 3 k$, ou seja, $2 k$ divisões na direção horizontal e $3 k$ na vertical, para $k=1, \cdots, 12$.

No estabelecimento das funções de aproximação, considerou-se a base $\beta_{0}$ para a aproximação do campo de deslocamentos nas fronteiras estáticas dos elementos e uma série de nove termos para a aproximação do campo de tensões no domínio de cada elemento das discretizações adotadas. Nas Figuras (7.4) e (7.5) encontram-se as curva obtidas que quantificam os erros relativos em porcentagem, respectivamente, da energia de deformação e do deslocamento horizontal do ponto de referência, em relação aos correspondentes valores de referência. Ressalta-se, ainda, que nas referidas figuras os graus de liberdade são resultantes da soma dos graus de liberdade em deslocamentos nodais e dos graus de liberdade em tensões.

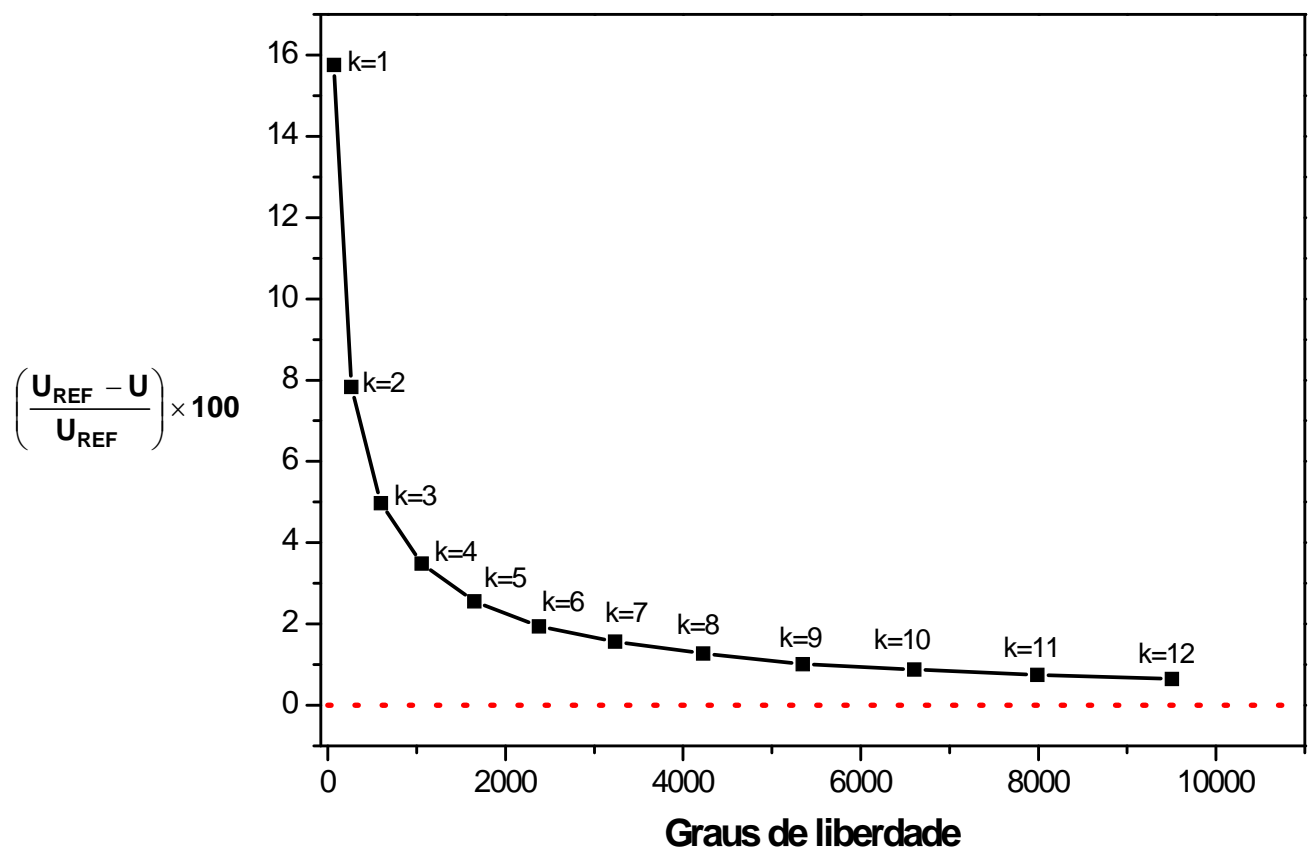

Figura 7.4. Refino-h. Erro relativo da energia de deformação (\%). 


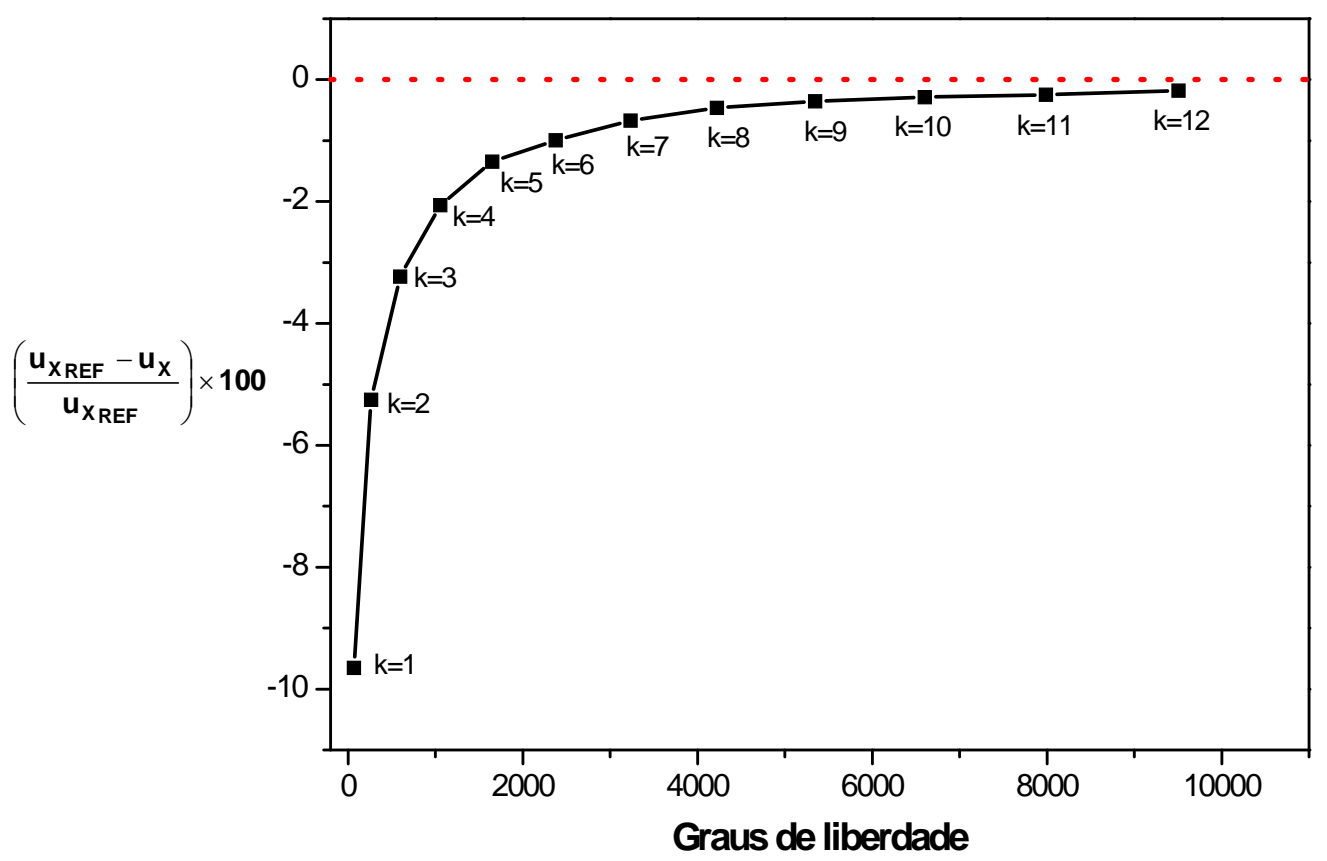

Figura 7.5. Refino-h. Erro relativo do deslocamento horizontal de referência (\%).

Percebe-se das Figuras (7.4) e (7.5) que a utilização de redes regulares levou a uma baixa taxa de convergência e que o número de graus de liberdade envolvido foi significativo.

A distribuição de tensões obtida para $k=12$ está ilustrada na Figura (7.6).

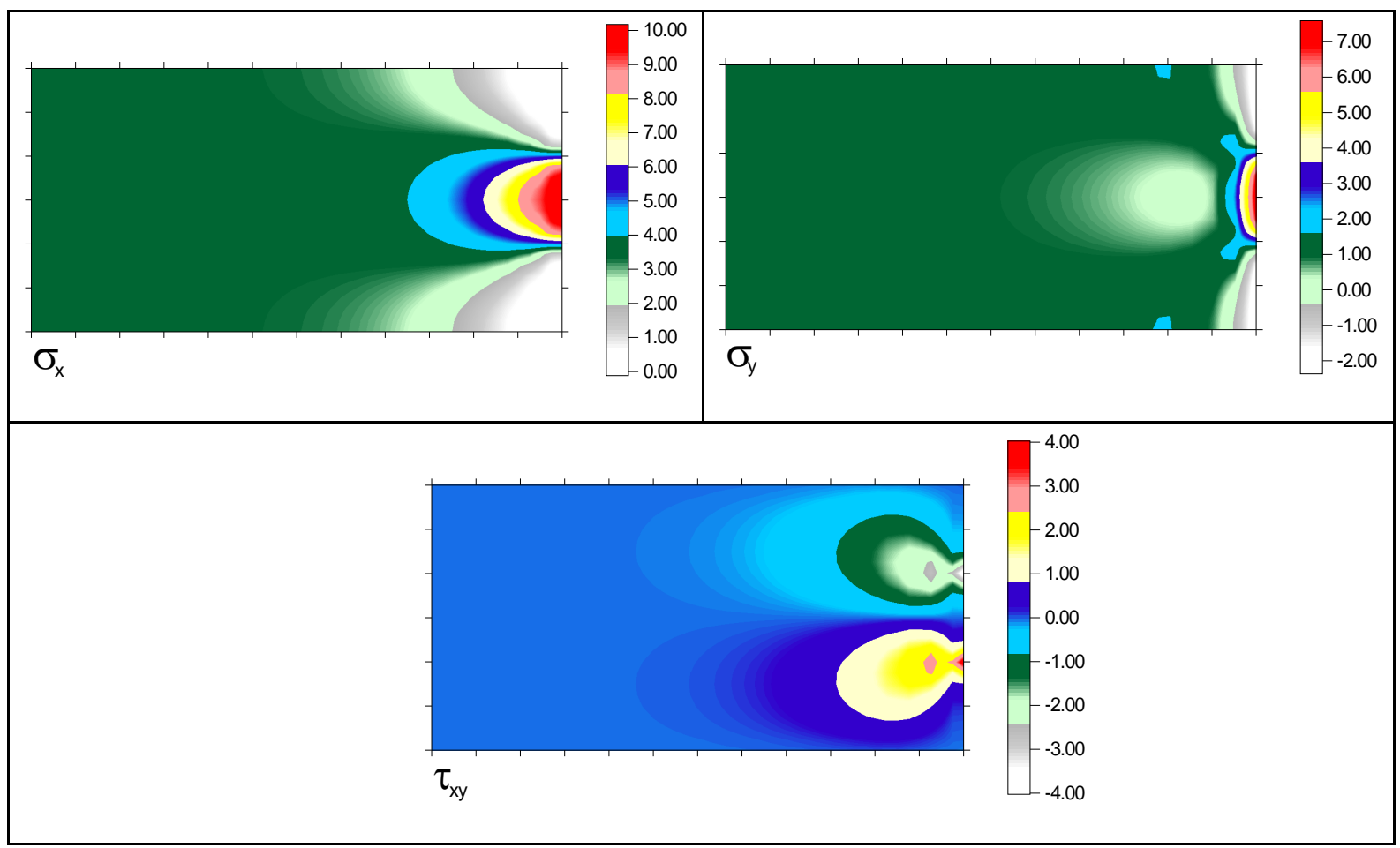

Figura 7.6. Distribuição de tensões para a rede regular $24 \times 36$.

Observa-se da Figura (7.6) uma distribuição de tensões conforme a esperada, com zonas de concentração de tensões na região próxima à aplicação do carregamento. Ela 
assemelhou-se à distribuição de referência, representada na Figura (7.3), mas o custo computacional envolvido foi demasiadamente superior.

Desta forma, infere-se que apesar da formulação estudada aproximar diretamente tensões, a adoção de redes inadequadas pode despender maior memória computacional e em certas ocasiões comprometer a qualidade da aproximação.

- Refino-p

No refino-p adotou-se uma rede regular $4 \times 6$, equivalente a $k=2$ da relação adotada no refino-h, conforme ilustrado na Figura (7.7).

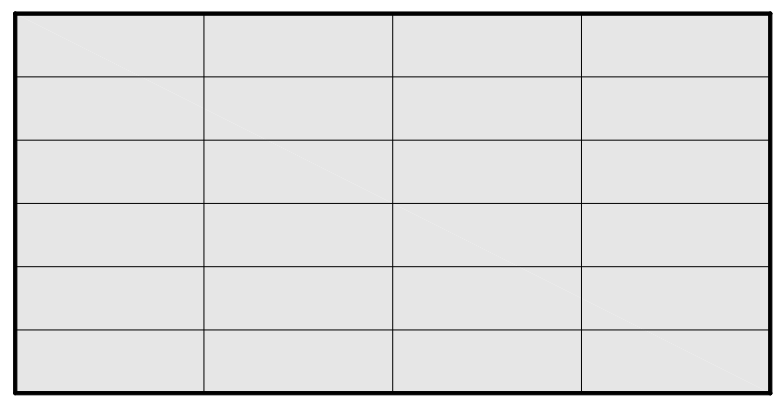

Figura 7.7. Discretização adotada no refino-p.

O conjunto de elementos e de fronteiras que foram enriquecidos no refino-p está indicado na Figura (7.8).

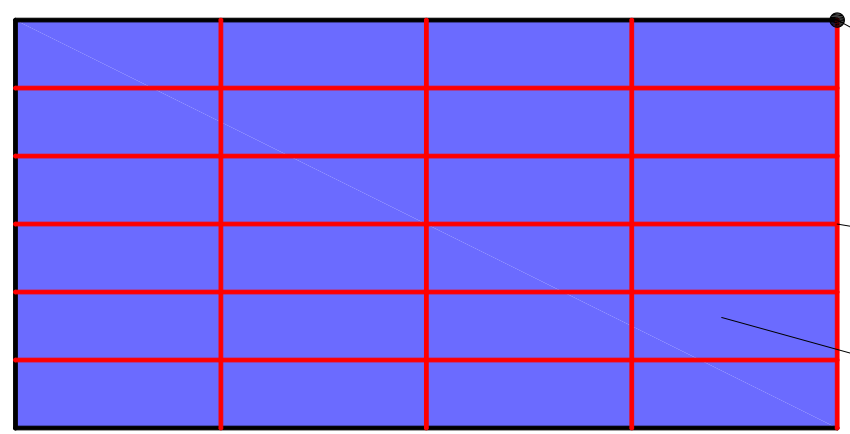

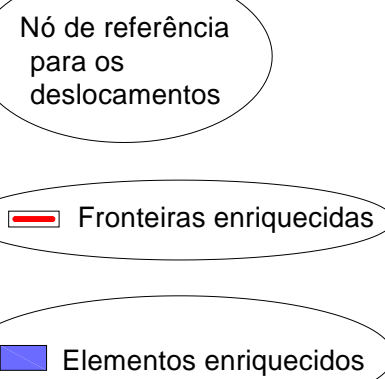

Elementos enriquecidos

Figura 7.8. Elementos e/ou fronteiras enriquecidos no refino-p.

Duas formas de refinamento foram realizadas. Na primeira manteve-se a base inicial $\beta_{0}$ de aproximação do campo de deslocamentos nas fronteiras estáticas dos elementos e expandiu-se a série de aproximação de tensões no domínio do elemento. Na segunda, tanto a aproximação do campo de deslocamentos quanto à aproximação de tensões foram enriquecidas, sempre atendendo ao teste do "mosaico". 
Na primeira forma de refino citada, os resultados obtidos estão indicados na Tabela (7.1).

Tabela 7.1 - Resultados do refino-p, quando se enriqueceu apenas o domínio dos elementos

\begin{tabular}{c|cc|c|c}
\hline \multirow{2}{*}{$\begin{array}{c}\text { Graus de } \\
\text { liberdade } \\
\text { em tensão } \\
\text { no } \Omega_{e}\end{array}$} & \multicolumn{2}{c|c}{$\begin{array}{c}\text { Energia de } \\
\text { deformação } \\
U_{R E F}=46,47\end{array}$} & \multicolumn{2}{c}{$\begin{array}{c}\text { Deslocamento horizontal } \\
\text { no ponto de referência } \\
u_{X R E F}=0,2818\end{array}$} \\
\cline { 2 - 5 } & Valor obtido & Erro relativo $(\%)$ & Valor obtido & Erro relativo (\%) \\
\hline 9 & 42,83 & 7,83 & 0,2965 & $-5,22$ \\
\hline 11 & 42,83 & 7,83 & 0,2965 & $-5,22$ \\
\hline 13 & 42,83 & 7,83 & 0,2966 & $-5,25$ \\
\hline 15 & 42,82 & 7,85 & 0,2966 & $-5,25$ \\
\hline 17 & 42,82 & 7,85 & 0,2966 & $-5,25$ \\
\hline 47 & 42,82 & 7,85 & 0,2967 & $-5,29$ \\
\hline
\end{tabular}

sendo $\Omega_{\mathrm{e}}$ o domínio de cada elemento.

Percebe-se claramente da Tabela (7.1) que o enriquecimento exclusivo sobre o campo de tensões nos domínios dos elementos não melhorou a qualidade das aproximações das grandezas consideradas. Os graus de liberdade foram despendidos inutilmente.

A distribuição de tensões obtida nesta forma de refino também apresentou baixa qualidade. Para a situação em que se considerou 47 graus de liberdade em tensão no domínio de cada elemento da discretização adotada, obteve-se a distribuição indicada na Figura (7.9).

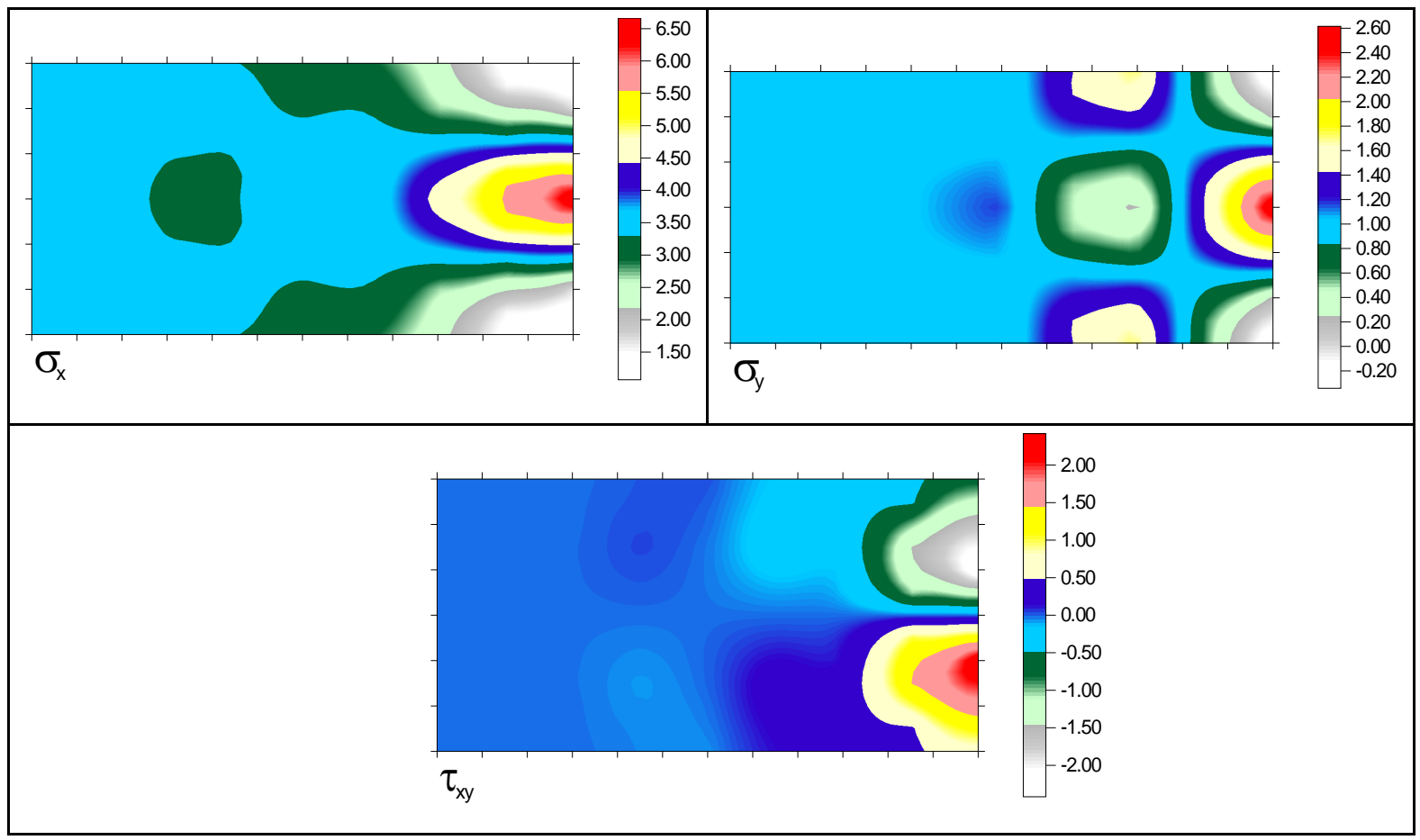

Figura 7.9. Distribuição de tensões obtida quando se enriqueceu apenas o domínio dos elementos.

Na segunda forma de refino exploraram-se as três bases de aproximação do campo de 
deslocamentos nas fronteiras estáticas dos elementos, já apresentadas: a base polinomial com refinamento hierárquico $\beta_{1}$, a polinomial não hierárquica $\beta_{2}$ e a não polinomial envolvendo funções trigonométricas $\beta_{3}$. As curvas obtidas que quantificam o erro relativo da energia de deformação e do deslocamento horizontal do ponto de referência estão representadas, respectivamente, nas Figuras (7.10) e (7.11). Nestas representações dim denota a dimensão das referidas bases, enquanto os graus de liberdade são resultados da soma dos graus de liberdade em tensão com os graus de liberdade em deslocamentos: nodais e decorrentes do enriquecimento. Esta notação será doravante utilizada em todas as análises futuras. A Tabela (7.2) quantifica os graus de liberdade envolvidos nas situações que foram analisadas. Ressalta-se, ainda, que o teste do mosaico sempre foi satisfeito.

Tabela 7.2 - Número total de graus de liberdade envolvidos no refino-p

\begin{tabular}{|l|c|c|c|c|c|c|c|} 
& \multicolumn{7}{|c|}{ Situações analisadas } \\
\hline Graus de liberdade em tensões no $\Omega_{e}$ & 9 & 15 & 21 & 27 & 33 & 39 & 45 \\
\hline Dimensão das bases em $\Gamma_{\text {te }}$ & 2 & 3 & 4 & 5 & 6 & 7 & 8 \\
\hline Total de graus de liberdade & 264 & 496 & 728 & 960 & 1192 & 1424 & 1656
\end{tabular}

sendo $\Omega_{\mathrm{e}}$ e $\Gamma_{\text {te }}$, respectivamente, o domínio e a fronteira estática de cada elemento da discretização adotada.

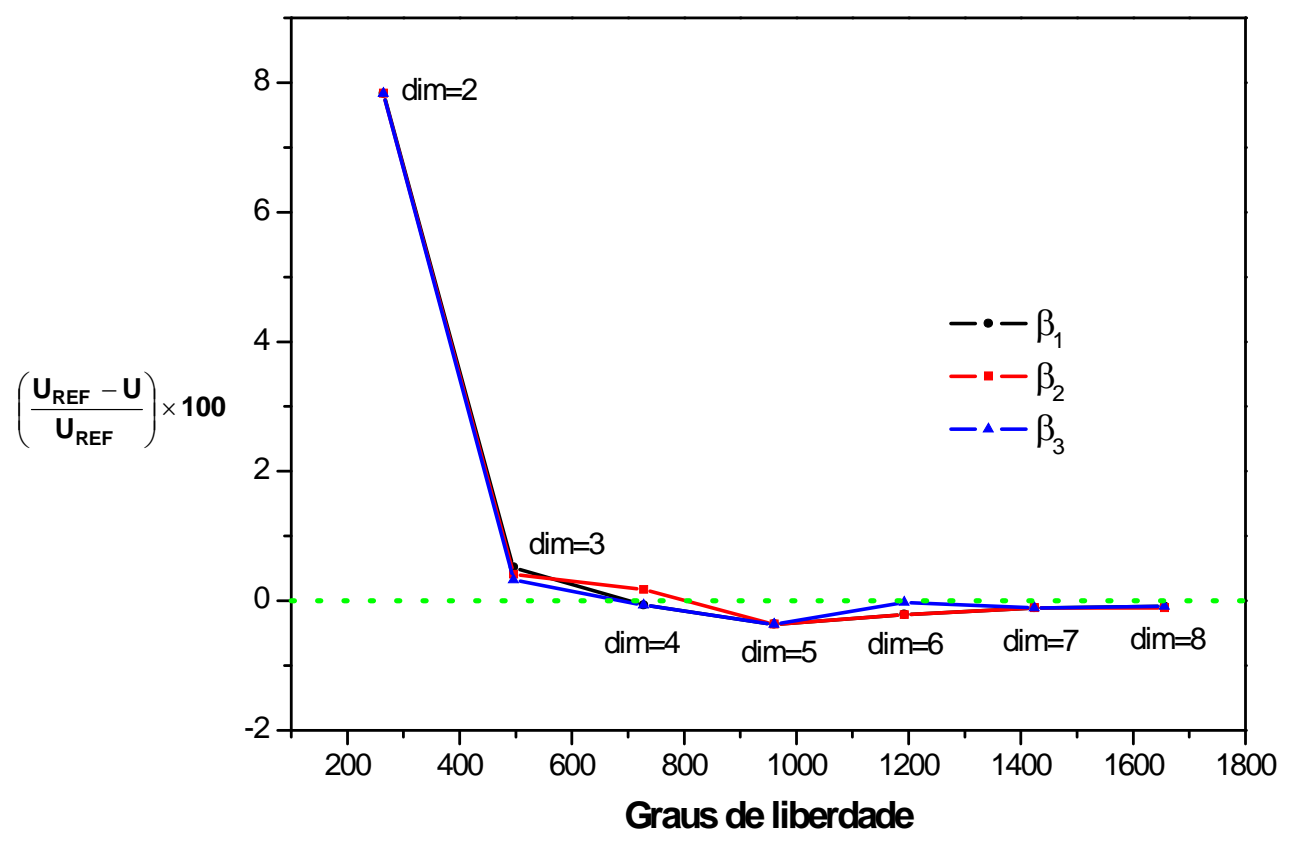

Figura 7.10. Refino-p. Erro relativo da energia de deformação (\%). 


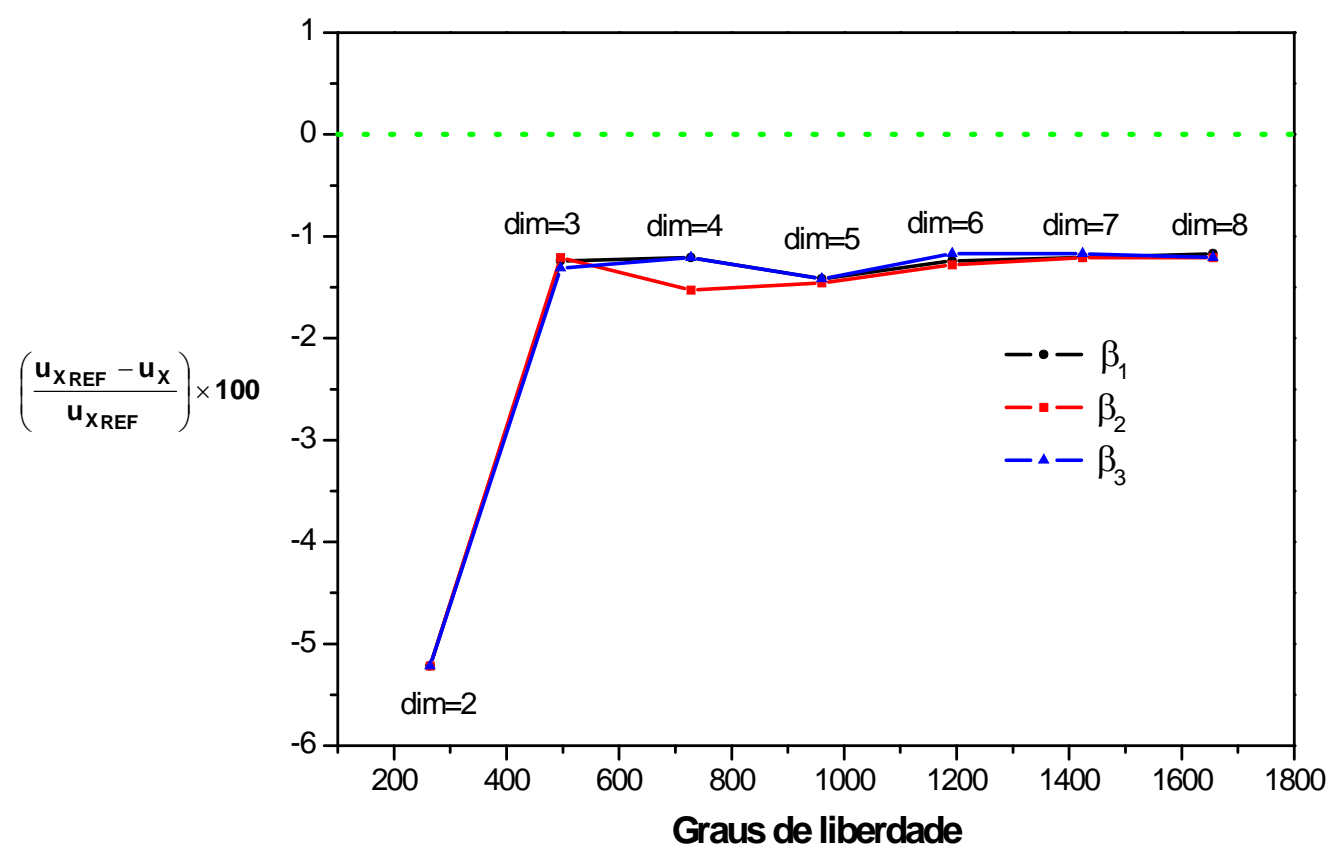

Figura 7.11. Refino-p. Erro relativo do deslocamento horizontal de referência (\%).

Observa-se nas curvas obtidas, Figuras (7.10) e (7.11), que houve uma mudança abrupta de configuração ao se passar da situação inicial, correspondente às bases $\beta_{1}, \beta_{2}$ e $\beta_{3}$ com dimensão igual a dois, para a situação seguinte de refino, onde se adotou bases de dimensão igual a três, indicando uma rápida taxa de convergência inicial. Nas situações seguintes a taxa de convergência foi menos acentuada, mas os valores aproximados tenderam para o valor de referência, com destaque para a energia de deformação (erro de 0,08 \%); apesar do reduzido número de graus de liberdade envolvidos, quando comparado a outras situações de refino-h já analisadas: via o MEF clássico ou por redes regulares da formulação híbrida-Trefftz. Além disto, verifica-se que não houve diferenças significativas entre as três bases utilizadas, atestando a versatilidade da formulação estudada.

No tocante à distribuição de tensões, praticamente não ocorreram diferenças apreciáveis entre as três bases analisadas. Por exemplo, para a base $\beta_{1}$ com $\operatorname{dim}=7$ obteve-se a representação indicada na Figura (7.12). 


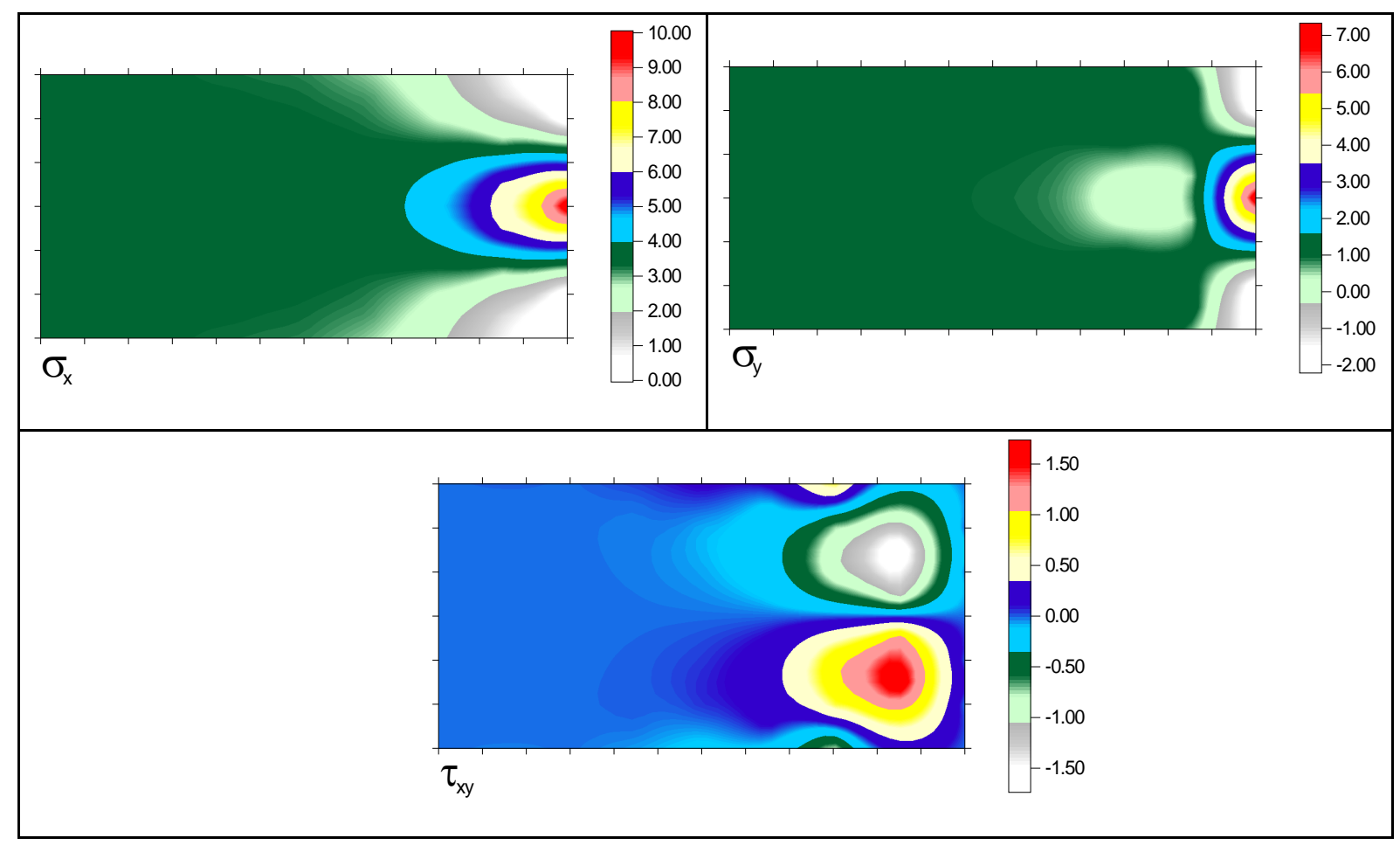

Figura 7.12. Distribuição de tensões no refino p com a base $\beta_{1} \operatorname{com} \operatorname{dim}=7$.

Comparado à distribuição de tensões obtidas sem o enriquecimento das fronteiras estáticas, representada na Figura (7.9), verificou-se que o refino-p, onde se enriqueceu concomitantemente as fronteiras estáticas e o domínio dos elementos, promoveu um aumento nas estimativas de tensões na região próxima à aplicação do carregamento, conforme ilustrado na Figura (7.12). No entanto, apesar da distribuição de tensões obtida, no referido refino-p, ter sido boa ela diferiu um pouco daquela de referência, representada na Figura (7.3). Isto se deve em parte às características de saída gráfica de tensões gerada pelo programa desenvolvido, a qual considera apenas a interpolação dos valores aproximados nos nós e no centróide dos elementos.

- Enriquecimento seletivo

Conforme já apresentado, a formulação apresentada neste trabalho contempla também o enriquecimento seletivo, permitindo-se priorizar porções de interesse no domínio estrutural. Em particular, neste problema, o enriquecimento seletivo restringiu-se ao entorno da região de aplicação do carregamento.

Neste contexto, consideraram-se duas discretizações, conforme ilustrado na Figura (7.13). Em cada uma delas exploraram-se algumas possibilidades de enriquecimento, no tocante tanto à região enriquecida considerada quanto às bases utilizadas nas fronteiras 
estáticas enriquecidas.

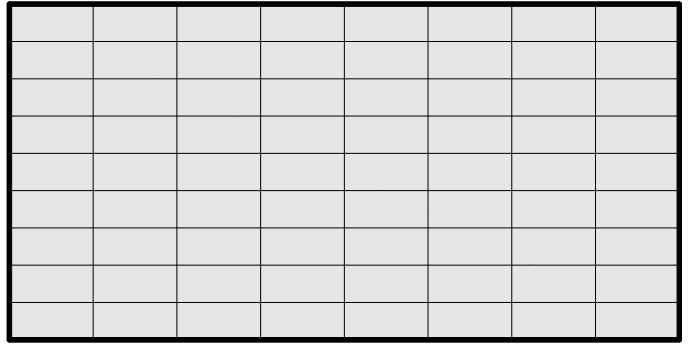

Rede regular $8 \times 9$

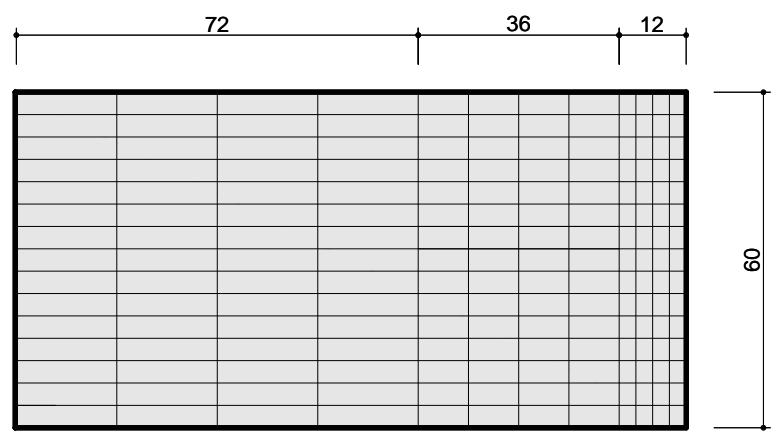

Rede irregular $12 \times 15$

Figura 7.13. Discretizações adotadas no enriquecimento seletivo.

Para a rede $8 \times 9$ consideraram-se duas situações de enriquecimento, concernentes à área enriquecida. A primeira abrangeu cinco elementos e nove fronteiras estáticas, enquanto a segunda envolveu o dobro das quantidades referidas na primeira situação, conforme ilustrado na Figura (7.14).

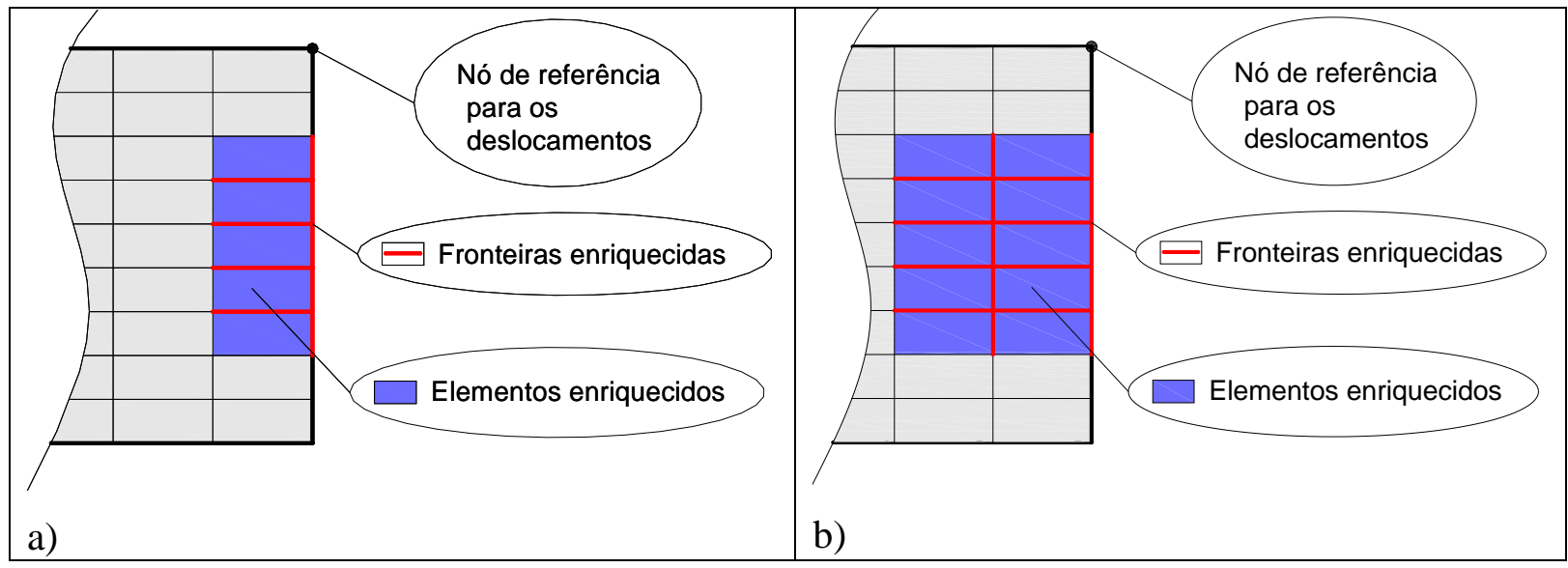

Figura 7.14. Região enriquecida da rede $8 \times 9$. a) Cinco elementos enriquecidos e nove fronteiras estáticas e b) Dez elementos enriquecidos e dezoito fronteiras estáticas.

$\mathrm{Na}$ Tabela (7.3) encontram-se os resultados obtidos de energia de deformação e deslocamento no ponto de referência, aproximados para algumas situações de enriquecimento consideradas. Logo em seguida, apresenta-se o total de graus de liberdade envolvidos referentes às situações analisadas, mediante a Tabela (7.4). 
Tabela 7.3 - Grandezas de interesse aproximadas para algumas situações de enriquecimento, quando se enriqueceu 5 elementos e 9 fronteiras estáticas da rede $8 \times 9$

\begin{tabular}{|c|c|c|c|c|c|c|}
\hline \multicolumn{3}{|c|}{ Condições de Enriquecimento } & \multicolumn{4}{|c|}{ Grandezas Aproximadas } \\
\hline \multirow{2}{*}{$\begin{array}{c}\text { Graus } \\
\text { de liberdade } \\
\text { em tensão no } \\
\Omega_{\mathrm{e}} \text { da } \\
\text { região } \\
\text { enriquecida }\end{array}$} & \multicolumn{2}{|c|}{$\begin{array}{c}\text { Base em } \Gamma_{\text {te }} \text { da } \\
\text { região } \\
\text { enriquecida }\end{array}$} & \multicolumn{2}{|c|}{$\begin{array}{c}\begin{array}{c}\text { Energia } \\
\text { de }\end{array} \\
\text { deformação } \\
U_{R E F}=46,47\end{array}$} & \multicolumn{2}{|c|}{$\begin{array}{c}\text { Deslocamento horizontal } \\
\text { no ponto de referência } \\
u_{X R E F}=0,2818\end{array}$} \\
\hline & Tipo & Dimensão & Valor obtido & $\begin{array}{c}\text { Erro } \\
\text { relativo } \\
(\%) \\
\end{array}$ & Valor obtido & $\begin{array}{c}\text { Erro } \\
\text { relativo } \\
(\%) \\
\end{array}$ \\
\hline 9 & $\beta_{0}$ & 2 & 44,69 & 3,83 & 0,2871 & $-1,88$ \\
\hline 27 & $\beta_{1}$ & 5 & 46,10 & 0,796 & 0,2854 & $-1,27$ \\
\hline 45 & $\beta_{1}$ & 8 & 46,09 & 0,817 & 0,2854 & $-1,27$ \\
\hline 27 & $\beta_{2}$ & 5 & 46,11 & 0,775 & 0,2854 & $-1,27$ \\
\hline 45 & $\beta_{2}$ & 8 & 46,10 & 0,796 & 0,2854 & $-1,27$ \\
\hline 27 & $\beta_{3}$ & 5 & 46,10 & 0,796 & 0,2854 & $-1,27$ \\
\hline 45 & $\beta_{3}$ & 8 & 46,09 & 0,817 & 0,2854 & $-1,27$ \\
\hline
\end{tabular}

sendo $\Omega_{\mathrm{e}}$ e $\Gamma_{\mathrm{te}}$ o domínio e a fronteira estática dos elementos, respectivamente.

Tabela 7.4 - Graus de liberdade envolvidos para algumas situações de enriquecimento, quando se enriqueceu 5 elementos e 9 fronteiras estáticas da rede $8 \times 9$

\begin{tabular}{|c|c|c|c|c|}
\hline \multicolumn{2}{|c|}{ Graus de liberdade em $\Omega_{\mathrm{e}}$ e base utilizada } & \multicolumn{2}{|c|}{ Dimensão da base em $\Gamma_{\text {te }}$} & \multirow{2}{*}{$\begin{array}{c}\text { Total de } \\
\text { graus de } \\
\text { liberdade }\end{array}$} \\
\hline $\begin{array}{c}\begin{array}{c}\text { Na região não } \\
\text { enriquecida }\end{array} \\
\end{array}$ & $\begin{array}{c}\text { Na região } \\
\text { enriquecida }\end{array}$ & $\begin{array}{c}\text { Na região não } \\
\text { enriquecida }\end{array}$ & $\begin{array}{c}\text { Na região } \\
\text { enriquecida }\end{array}$ & \\
\hline $9-\beta_{0}$ & $9-\beta_{0}$ & 2 & 2 & 792 \\
\hline $9-\beta_{0}$ & $27-\beta_{1}, \beta_{2}$ ou $\beta_{3}$ & 2 & 5 & 936 \\
\hline $9-\beta_{0}$ & $45-\beta_{1}, \beta_{2}$ ou $\beta_{3}$ & 2 & 8 & 1080 \\
\hline
\end{tabular}

Verifica-se a partir da Tabela (7.3) que o enriquecimento promoveu melhorias nas aproximações das grandezas de interesse, as quais apresentaram valores próximos dos esperados; apesar do reduzido número de graus de liberdades envolvidos nas situações consideradas, conforme ilustrado na Tabela (7.4). O número de graus de liberdade na situação de maior dispêndio foi inferior a um terço dos graus de liberdade considerados na análise via o MEF clássico. Além disso, percebe-se que os resultados obtidos com as três bases utilizadas foram muito próximos.

As distribuições de tensões obtidas no enriquecimento com as três bases consideradas também não apresentaram diferenças significativas. $\mathrm{Na}$ figura (7.15), ilustra-se as representações de tensões na situação que não foi contemplada pelo enriquecimento e naquela que envolveu 936 graus de liberdade e utilizou a base $\beta_{2}$. 


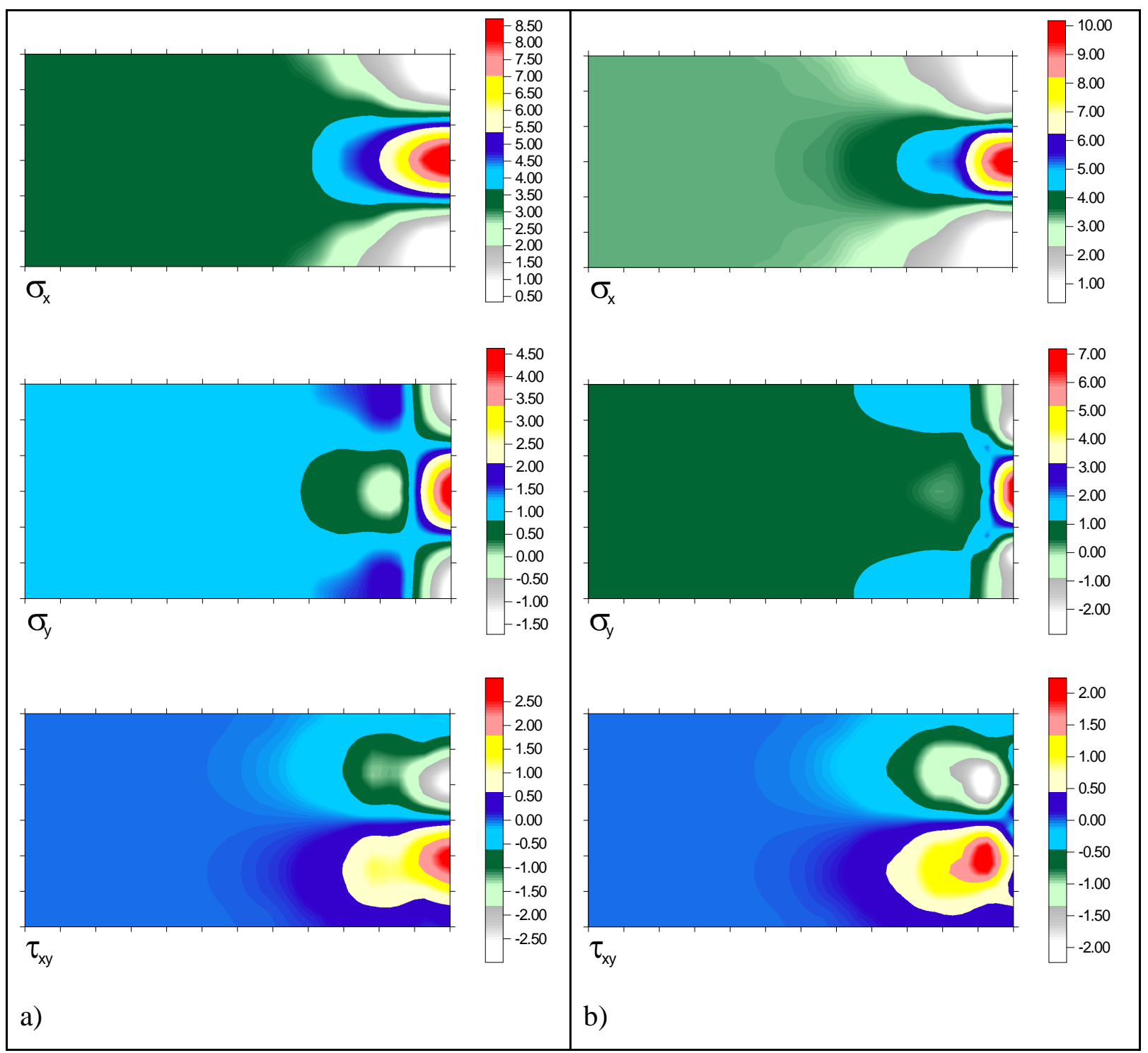

Figura 7.15. Distribuição de tensões. a) Situação não enriquecida e b) Enriquecimento com a base $\beta_{2}$ com $\operatorname{dim}=5$.

Da figura (7.15) percebe-se que o enriquecimento seletivo promoveu um aumento nas estimativas de tensões nas zonas próximas à aplicação do carregamento. Além disso, a distribuição obtida assemelhou-se com a distribuição de referência (vide Figura (7.3)).

Na segunda opção de enriquecimento considerado para a rede 8x9 os resultados foram ainda melhores, como se pode observar na Tabela (7.5). Evidentemente, nesta situação, o número de graus liberdade envolvidos foi superior, conforme ilustrado na Tabela (7.6); mas ainda aquém dos utilizados numa análise via o MEF convencional. 
Tabela 7.5 - Grandezas de interesse aproximadas para algumas situações de enriquecimento, quando se enriqueceu 10 elementos e 18 fronteiras estáticas da rede 8x9

\begin{tabular}{|c|c|c|c|c|c|c|}
\hline \multicolumn{3}{|c|}{ Condições de Enriquecimento } & \multicolumn{4}{|c|}{ Grandezas Aproximadas } \\
\hline \multirow{2}{*}{$\begin{array}{c}\text { Graus } \\
\text { de liberdade } \\
\text { em tensão no } \\
\Omega_{\mathrm{e}} \text { da } \\
\text { região } \\
\text { enriquecida }\end{array}$} & \multicolumn{2}{|c|}{$\begin{array}{c}\text { Base em } \Gamma_{\text {te }} \text { da } \\
\text { região } \\
\text { enriquecida }\end{array}$} & \multicolumn{2}{|c|}{$\begin{array}{c}\text { Energia de } \\
\text { deformação } \\
U_{R E F}=46,47\end{array}$} & \multicolumn{2}{|c|}{$\begin{array}{c}\text { Deslocamento horizonta } \\
\text { no ponto de referência } \\
u_{X R E F}=0,2818\end{array}$} \\
\hline & Tipo & Dimensão & Valor obtido & $\begin{array}{c}\text { Erro } \\
\text { relativo } \\
(\%)\end{array}$ & Valor obtido & $\begin{array}{c}\text { Erro } \\
\text { relativo } \\
(\%)\end{array}$ \\
\hline 9 & $\beta_{0}$ & 2 & 44,69 & 3,83 & 0,2871 & $-1,88$ \\
\hline 27 & $\beta_{1}$ & 5 & 46,19 & 0,602 & 0,2848 & $-1,06$ \\
\hline 45 & $\beta_{1}$ & 8 & 46,17 & 0,645 & 0,2849 & $-1,10$ \\
\hline 27 & $\beta_{2}$ & 5 & 46,19 & 0,602 & 0,2848 & $-1,06$ \\
\hline 45 & $\beta_{2}$ & 8 & 46,18 & 0,624 & 0,2849 & $-1,10$ \\
\hline 27 & $\beta_{3}$ & 5 & 46,19 & 0,602 & 0,2848 & $-1,06$ \\
\hline 45 & $\beta_{3}$ & 8 & 46,17 & 0,645 & 0,2849 & $-1,10$ \\
\hline
\end{tabular}

Tabela 7.6 - Graus de liberdade envolvidos para algumas situações de enriquecimento, quando se enriqueceu 10 elementos e 18 fronteiras estáticas da rede 8x9

\begin{tabular}{|c|c|c|c|c|}
\hline \multicolumn{2}{|c|}{ Graus de liberdade em $\Omega_{\mathrm{e}}$ e base utilizada } & \multicolumn{2}{|c|}{ Dimensão da base em $\Gamma_{\text {te }}$} & \multirow{2}{*}{$\begin{array}{l}\text { Total de } \\
\text { graus de } \\
\text { liberdade }\end{array}$} \\
\hline $\begin{array}{c}\text { Na região não } \\
\text { enriquecida }\end{array}$ & $\begin{array}{c}\text { Na região } \\
\text { enriquecida }\end{array}$ & $\begin{array}{c}\text { Na região não } \\
\text { enriquecida }\end{array}$ & $\begin{array}{c}\text { Na região } \\
\text { enriquecida }\end{array}$ & \\
\hline $9-\beta_{0}$ & $9-\beta_{0}$ & 2 & 2 & 792 \\
\hline $9-\beta_{0}$ & $27-\beta_{1}, \beta_{2}$ ou $\beta_{3}$ & 2 & 5 & 1080 \\
\hline $9-\beta_{0}$ & $45-\beta_{1}, \beta_{2}$ ou $\beta_{3}$ & 2 & 8 & 1368 \\
\hline
\end{tabular}

A distribuição de tensões obtidas foi praticamente idêntica à representada na Figura (7.15 b).

Finalmente, uma última situação de enriquecimento foi averiguada. Ela combinou aspectos dos refinos $\mathrm{p}$ e h no entorno da região de aplicação do carregamento. A região enriquecida foi formada por quatorze elementos e vinte e seis fronteiras estáticas, conforme ilustrado na Figura (7.16).

Nesta circunstância os resultados obtidos se aproximaram muito dos valores de referência, como se pode observar na Tabela (7.7). Os graus de liberdade envolvidos, nesta última análise ainda foram inferiores aos utilizados na análise via o MEF clássico, conforme indicado na Tabela (7.8). 


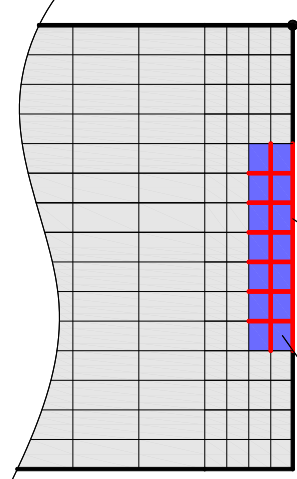

Nó de referência

para os

deslocamentos

Fronteiras enriquecidas

Elementos enriquecidos

Figura 7.16. Região enriquecida da rede irregular $12 \times 15$

Tabela 7.7 - Grandezas de interesse aproximadas para algumas situações de enriquecimento, quando se enriqueceu 14 elementos e 26 fronteiras estáticas da rede $12 \times 15$

\begin{tabular}{c|c|c|c|c|c|c}
\hline \multicolumn{2}{c|}{ Condições de Enriquecimento } & \multicolumn{4}{c}{ Grandezas Aproximadas } \\
\hline $\begin{array}{c}\text { Graus } \\
\text { de liberdade } \\
\text { em tensão no } \\
\Omega_{\mathrm{e} \text { da }} \\
\text { região } \\
\text { enriquecida }\end{array}$ & $\begin{array}{c}\text { Base em } \Gamma_{\text {te }} \text { da } \\
\text { região } \\
\text { enriquecida }\end{array}$ & \multicolumn{2}{c}{$\begin{array}{c}\text { Energia de } \\
\text { deformação } \\
U_{R E F}=46,47\end{array}$} & $\begin{array}{c}\text { Deslocamento horizontal } \\
\text { no ponto de referência } \\
u_{\text {XREF }}=0,2818\end{array}$ \\
\cline { 2 - 7 } & Tipo & Dimensão & Valor obtido & $\begin{array}{c}\text { Erro } \\
\text { relativo } \\
(\boldsymbol{\%})\end{array}$ & Valor obtido & $\begin{array}{c}\text { Erro } \\
\text { relativo } \\
(\boldsymbol{\%})\end{array}$ \\
\hline 9 & $\beta_{0}$ & 2 & 46,08 & 0,839 & 0,2821 & $-0,106$ \\
\hline 27 & $\beta_{1}$ & 5 & 46,34 & 0,279 & 0,2820 & $-0,070$ \\
\hline 45 & $\beta_{1}$ & 8 & 46,34 & 0,279 & 0,2820 & $-0,070$ \\
\hline 27 & $\beta_{2}$ & 5 & 46,34 & 0,279 & 0,2820 & $-0,070$ \\
\hline 45 & $\beta_{2}$ & 8 & 46,34 & 0,279 & 0,2820 & $-0,070$ \\
\hline 27 & $\beta_{3}$ & 5 & 46,34 & 0,279 & 0,2820 & $-0,070$ \\
\hline 45 & $\beta_{3}$ & 8 & 46,34 & 0,279 & 0,2820 & $-0,070$ \\
\hline
\end{tabular}

Tabela 7.8-Graus de liberdade envolvidos para algumas situações de enriquecimento, quando se enriqueceu 14 elementos e 26 fronteiras estáticas da rede $12 \times 15$

\begin{tabular}{|c|c|c|c|c|}
\hline \multicolumn{2}{|c|}{ Graus de liberdade em $\Omega_{\mathrm{e}}$ e base utilizada } & \multicolumn{2}{|c|}{ Dimensão da base em $\Gamma_{\text {te }}$} & \multirow{2}{*}{$\begin{array}{c}\text { Total de } \\
\text { graus de } \\
\text { liberdade }\end{array}$} \\
\hline $\begin{array}{c}\text { Na região não } \\
\text { enriquecida }\end{array}$ & $\begin{array}{c}\text { Na região } \\
\text { enriquecida }\end{array}$ & $\begin{array}{c}\text { Na região não } \\
\text { enriquecida }\end{array}$ & $\begin{array}{c}\text { Na região } \\
\text { enriquecida }\end{array}$ & \\
\hline $9-\beta_{0}$ & $9-\beta_{0}$ & 2 & 2 & 1980 \\
\hline $9-\beta_{0}$ & $27-\beta_{1}, \beta_{2}$ ou $\beta_{3}$ & 2 & 5 & 2388 \\
\hline $9-\beta_{0}$ & $45-\beta_{1}, \beta_{2}$ ou $\beta_{3}$ & 2 & 8 & 2796 \\
\hline
\end{tabular}

As distribuições de tensões obtidas para a situação não enriquecida e enriquecida com a base $\beta_{3}$, envolvendo 2796 graus de liberdade, estão indicadas na Figura (7.17). 


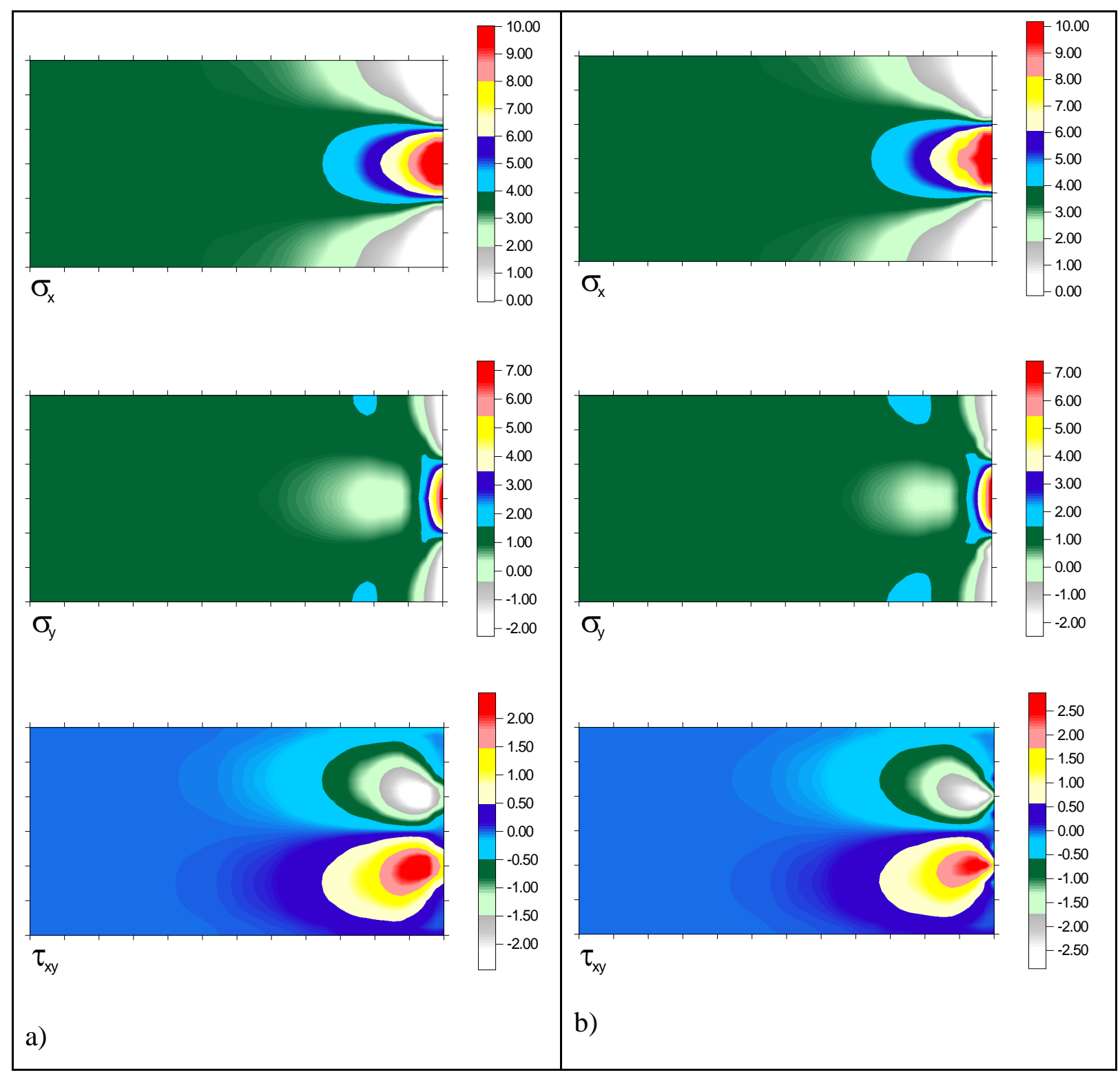

Figura 7.17. Distribuição de tensões. a) Situação não enriquecida e b) Enriquecimento com a base $\beta_{3}$ com $\operatorname{dim}=8$.

Verifica-se que o enriquecimento não promoveu alterações significativas na distribuição de tensões, uma vez que ainda na situação não enriquecida se obtiveram valores apreciáveis.

Ressalta-se que nesta última análise os resultados poderiam ser ainda melhores, caso se adotasse uma rede com um refinamento mais adequado no entorno da região de aplicação do carregamento (por exemplo, tomando-se uma faixa horizontal, na qual incidiria o refino-h, com dimensões da ordem de grandeza da extensão do carregamento). 


\section{3 $2^{\circ}$ Problema: análise de distorção da rede}

O segundo problema trata de uma chapa sob certas condições de vinculação submetida a um carregamento uniformemente distribuído $(W=10)$ sobre a aresta superior. A chapa encontra-se impedida de se deslocar na aresta esquerda segundo as direções $x$ e $y$ $\left(u_{x}=u_{y}=0\right)$. Estes e outros detalhes estão indicados na Figura (7.18).

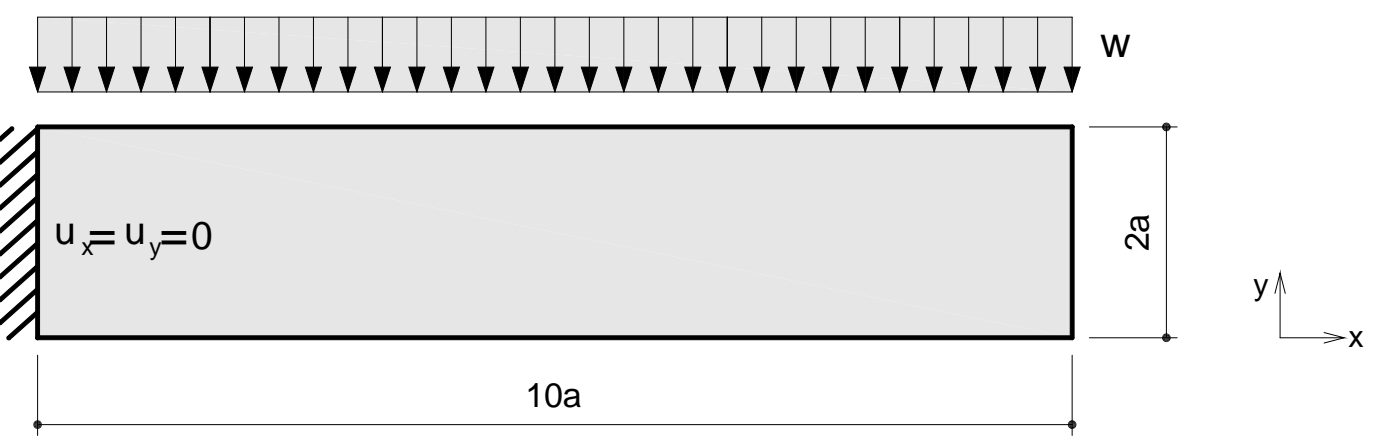

Figura 7.18. Chapa analisada no $2^{\circ}$ problema. Sua geometria, carregamento e vinculação.

No dimensionamento da referida chapa adotou-se $a=12$ e espessura unitária. As propriedades de interesse do material apresentam os seguintes valores: módulo de Young $E$ igual a 100000 e coeficiente de Poisson $v$ igual a 0,3.

Na obtenção dos valores de referência de energia de deformação e distribuição de tensões realizou-se uma análise via o MEF clássico com o auxílio do software ANSYS ${ }^{\circledR}$. Em tal análise adotou-se uma discretização composta de 736 elementos idênticos ao do $1^{\circ}$ problema, envolvendo 1569 nós, com um total de 3138 graus de liberdade. Na figura (7.19) encontra-se a distribuição dos elementos da discretização adotada.

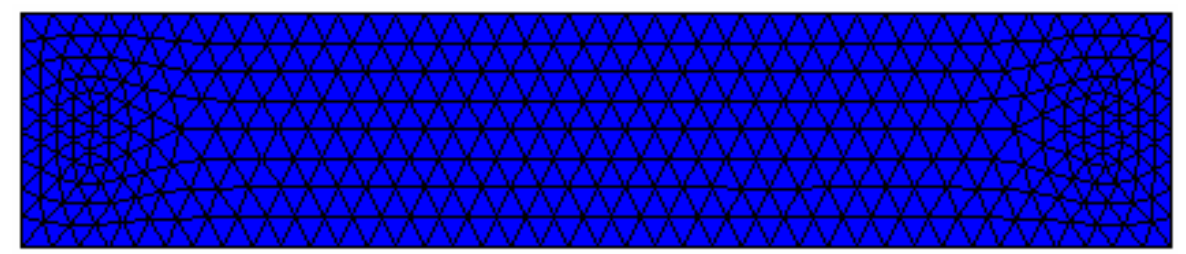

Figura 7.19. Discretização adotada na análise realizada via o MEF clássico para obtenção dos valores de referência do $2^{\circ}$ problema.

Obteve-se como referência para a energia de deformação $U_{R E F}$ o valor de 577,68. Por sua vez a distribuição de tensões de referência obtida pode ser visualizada na Figura (7.20). 


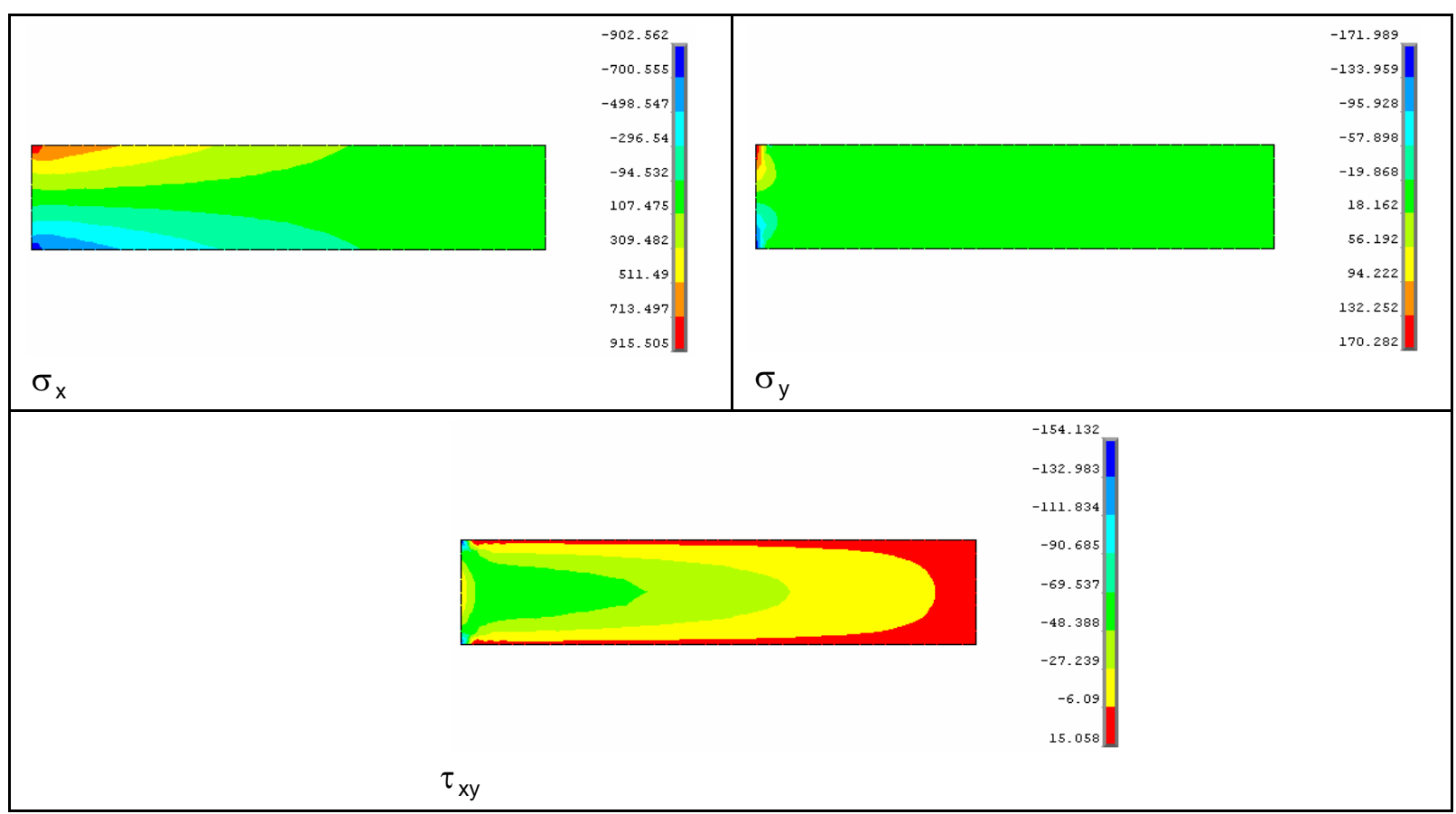

Figura 7.20. Distribuição de tensões de referência do $2^{\circ}$ problema.

Com o propósito de avaliar a robustez do elemento da formulação estudada utilizou-se um procedimento semelhante ao proposto por Bussamra (1999), onde se avaliam algumas grandezas de interesse para um modelo tridimensional muito simples de uma viga. Tal modelo é constituído por dois elementos que podem sofrer alterações apreciáveis de forma.

Neste trabalho o procedimento citado foi adaptado para uma discretização composta por oito elementos, os quais podem sofrer alterações de formato, a depender da distância de distorção $\mu$ considerada, conforme ilustrado na Figura (7.21). Para $\mu=0$ obtém-se uma rede regular sem nenhuma distorção, onde todos os elementos são retangulares, enquanto para $\mu=0,25$ a distorção é máxima, situação em que os elementos quadrilaterais se degeneram em elementos triangulares. Nas situações intermediárias, onde $0<\mu<0,25$, a rede é formada por oito elementos distorcidos.

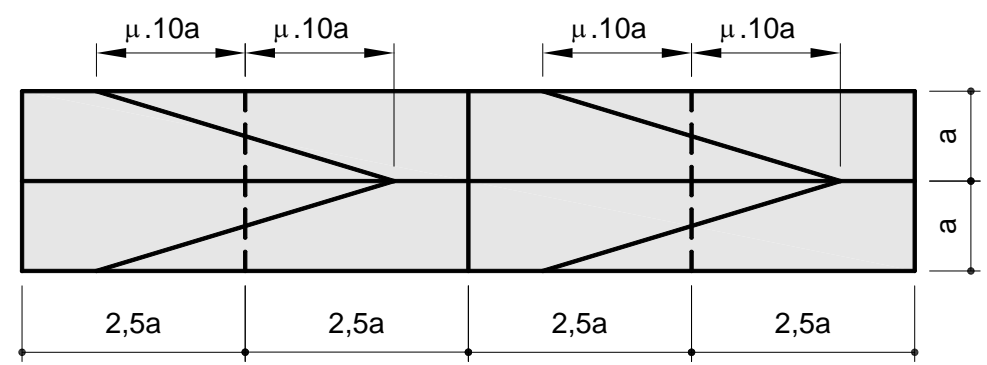

Figura 7.21. Discretização adotada na análise do $2^{\circ}$ problema.

Desta forma, primeiramente, realizou-se uma análise de convergência da energia de deformação para a rede não distorcida $(\mu=0)$, envolvendo o refino-p das três bases de 
aproximação do campo de deslocamentos exploradas neste trabalho: $\beta_{1}, \beta_{2}$, e $\beta_{3}$. Em seguida, foram realizados alguns testes de distorção de rede com valores de distorção $\mu$ variando entre 0 e 0,249 , onde se confrontaram os valores não distorcidos com os distorcidos para diferentes dimensões da base $\beta_{1}$. Em todas as avaliações o enriquecimento incidiu sobre todos os elementos da discretização adotada e sobre algumas fronteiras estáticas, conforme ilustrado na Figura (7.22). Neste contexto ressalta-se que o teste do "mosaico" foi uma condição preestabelecida e imprescindível para a convergência.

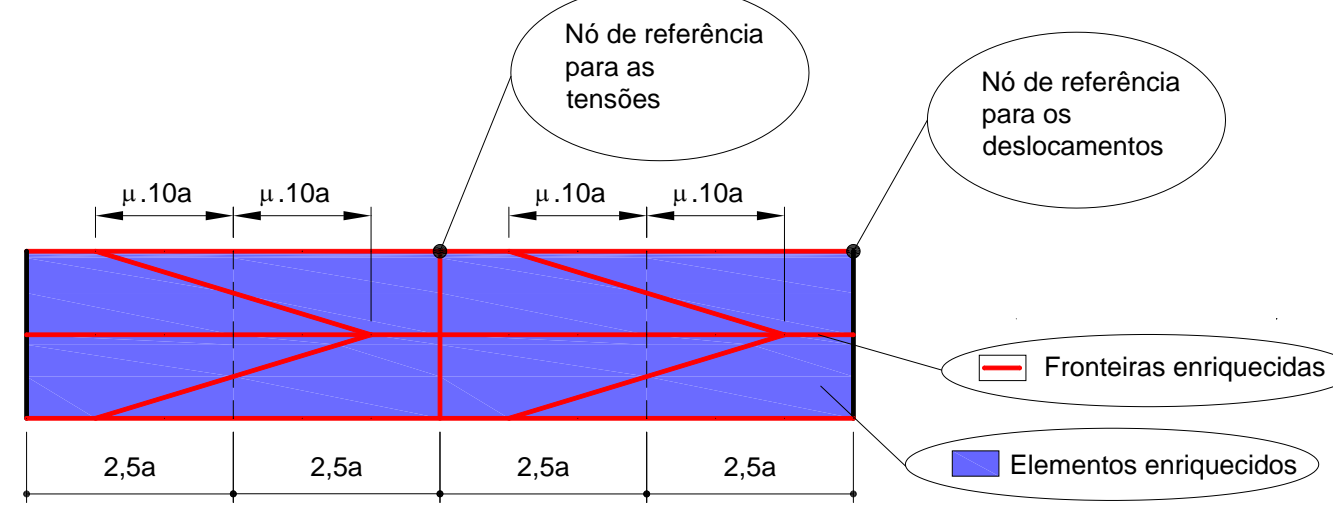

Figura 7.22. Elementos e fronteiras estáticas enriquecidos na discretização adotada.

A curva obtida que quantifica o erro relativo da energia de deformação pode ser visualizada na Figura (7.23), onde se constata nitidamente a convergência das três bases utilizadas para o valor de referência (erro de $0,05 \%$ ); mesmo para o baixo número de graus de liberdade envolvidos (cerca de um quinto dos utilizados na análise via o MEF clássico).

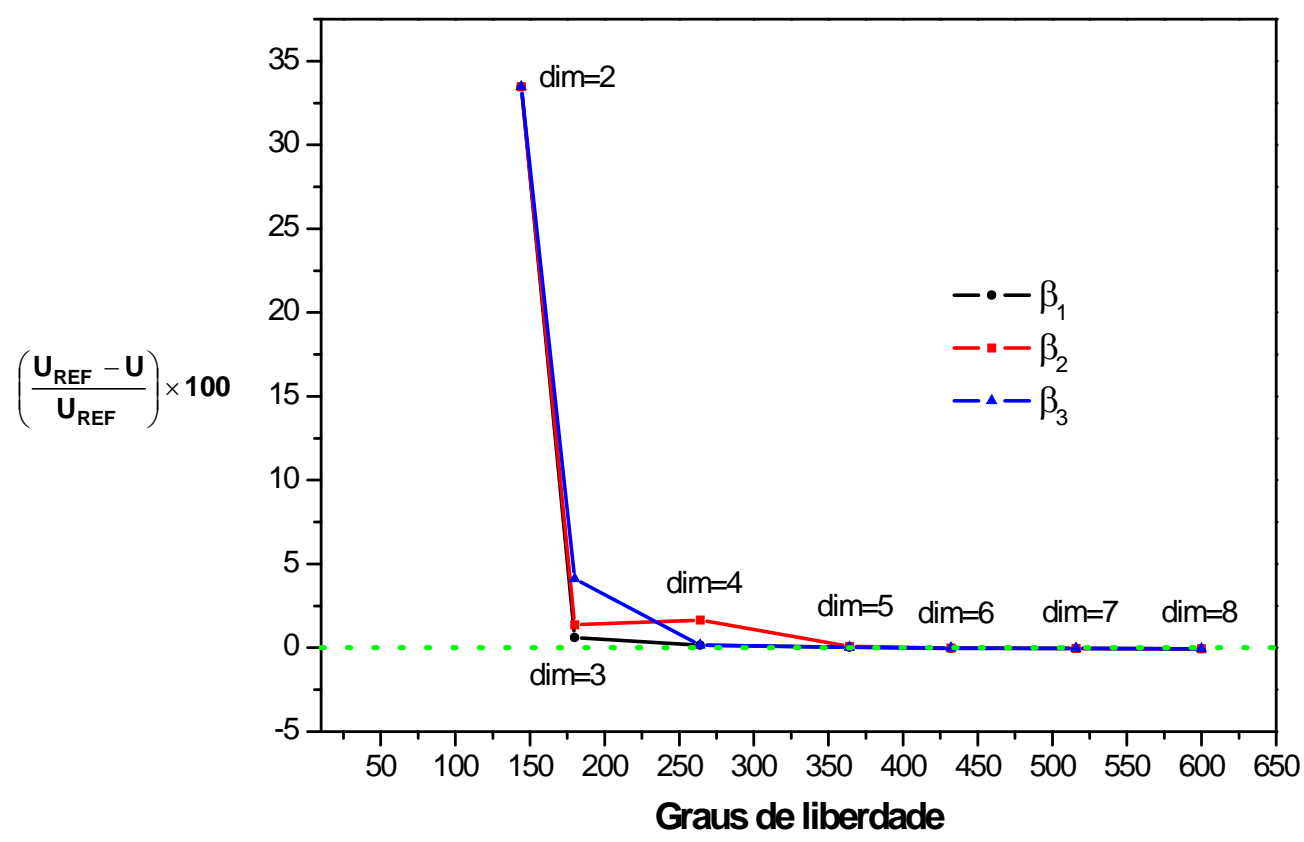

Figura 7.23. Refino-p. Erro relativo da energia de deformação (\%). 
Por sua vez, observa-se que as distribuições de tensões obtidas para as três bases utilizadas foram praticamente idênticas, mas apresentaram valores ligeiramente discrepantes daqueles correspondentes à distribuição de referência, conseqüência provável da saída gráfica de tensões, a qual envolve somente os nós e o centro de gravidade dos elementos da discretização adotada. Por exemplo, para a base $\beta_{2}$, numa situação que envolveu 600 graus de liberdade, obteve-se a representação indicada na Figura (7.24).

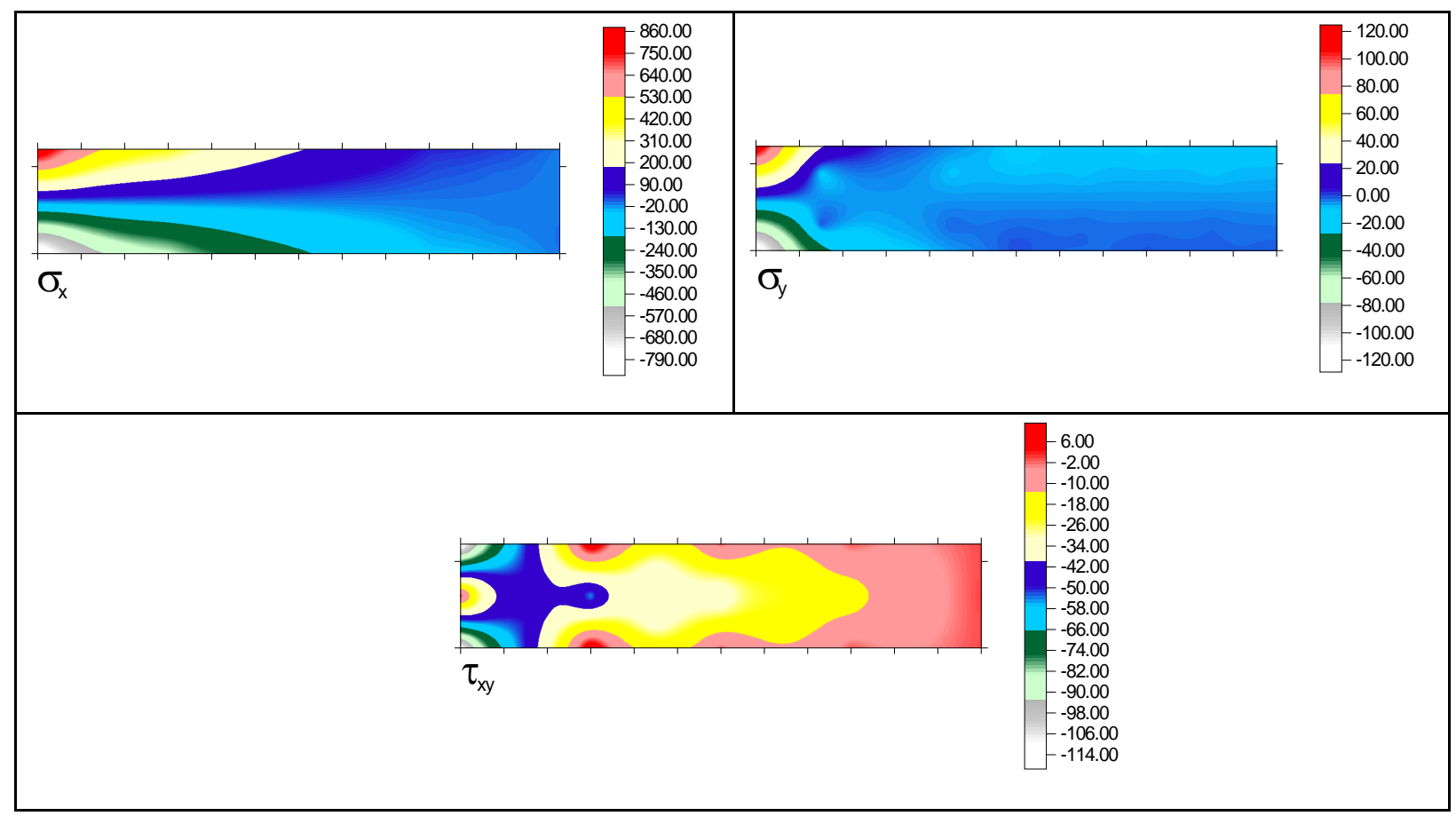

Figura 7.24. Distribuição de tensões obtida no refino-p com a base $\beta_{2}$ com dim $=7$.

Nos testes de distorção da rede aumentou-se gradativamente a distância de distorção $\mu$ e analisaram-se as alterações nos valores de energia de deformação, deslocamento e tensões em pontos de referência (vide Figura (7.22)), para diferentes situações de refino da base $\beta_{1}$. As curvas foram construídas a partir de valores de distorção como abscissas $(0 \leq \mu \leq 0,249)$ e das referidas grandezas normalizadas como ordenadas. Cada valor normalizado foi obtido dividindo-se a grandeza analisada para certo nível de distorção da rede pelo respectivo valor obtido da rede não distorcida.

Os resultados obtidos nos testes de sensibilidade à distorção para a energia de deformação, tensões e deslocamentos em pontos de referência podem ser visualizados, respectivamente, nas Figuras (7.25), (7.26) e (7.27). Em todas elas verifica-se que apenas a rede não enriquecida $\left(\beta_{1} \operatorname{com} \operatorname{dim}=2\right)$ apresentou uma sensibilidade significativa à distorção da rede. Na maioria das situações analisadas, onde se considerou o enriquecimento da rede, os 
elementos mostraram-se extremamente robustos, mesmo em situações extremas de distorção ( $\mu=0,248$ ). Para $\mu=0,249$, quando do enriquecimento de $\beta_{1}$ com dim=8, verificou-se variações abruptas das grandezas analisadas, consequiência provável de uma instabilidade numérica por perda de precisão.

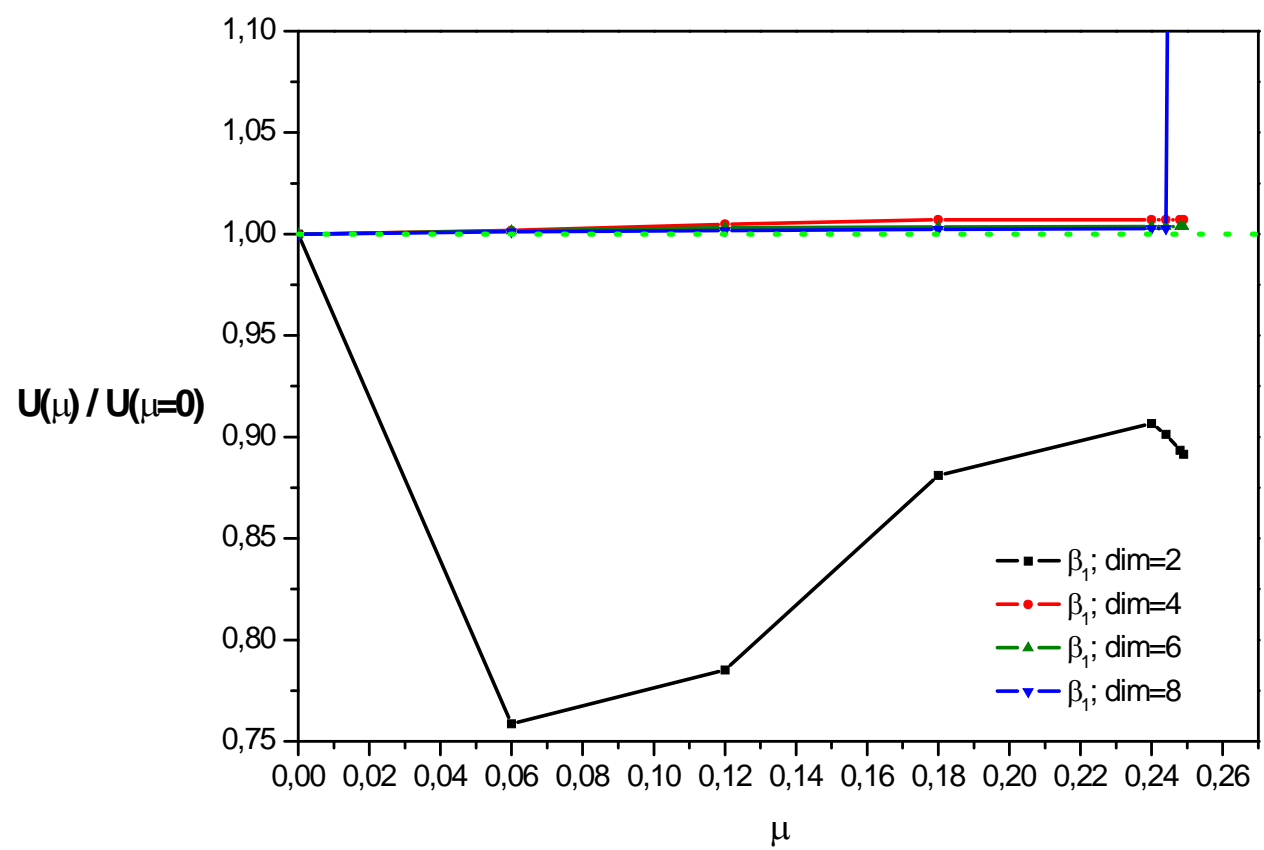

Figura 7.25. Sensibilidade da energia de deformação à distorção da rede.

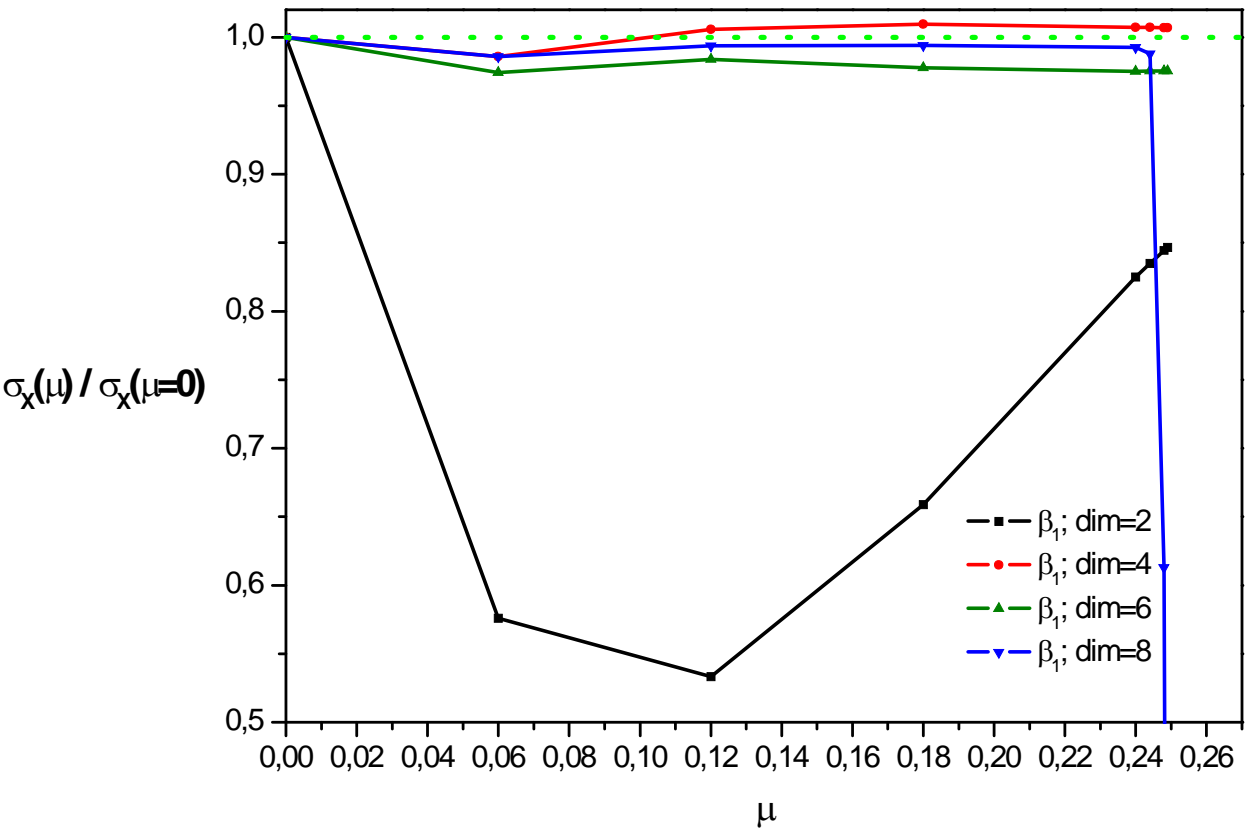

Figura 7.26. Sensibilidade da tensão de referência à distorção da rede. 


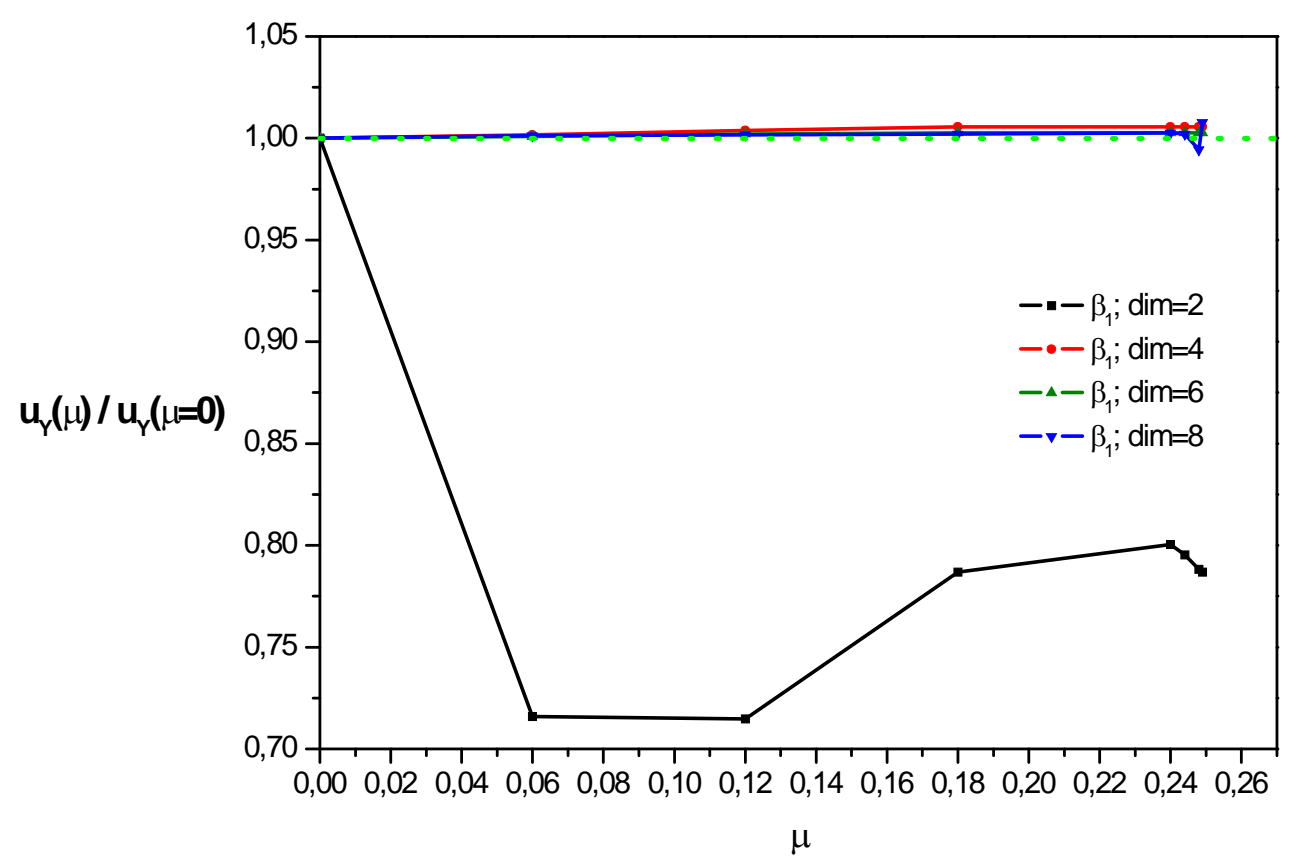

Figura 7.27. Sensibilidade do deslocamento vertical de referência à distorção da rede.

\section{4 $3^{\circ}$ Problema: chapa com um orifício circular excêntrico}

O terceiro problema refere-se a uma chapa com um orifício excêntrico submetida a certas condições de carregamento e vinculação, conforme ilustrado na Figura (7.28).

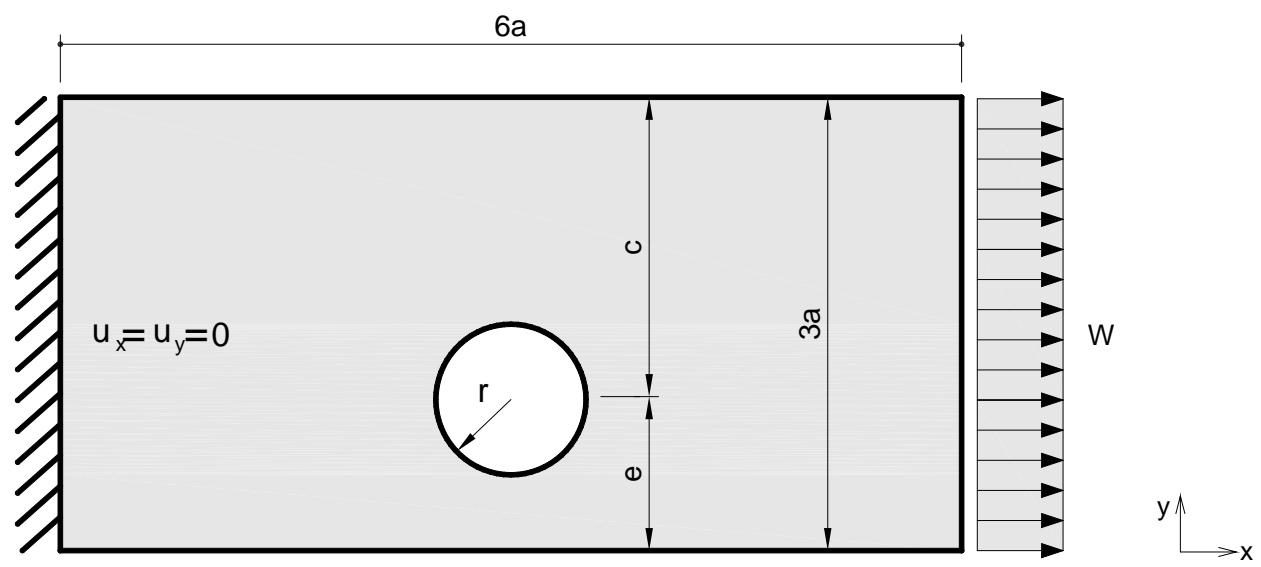

Figura 7.28. Chapa analisada no $3^{\circ}$ problema. Sua geometria, carregamento e vinculação.

A referida chapa encontra-se impedida de deslocar-se na aresta esquerda segundo as direções $x$ e $y\left(u_{x}=u_{y}=0\right)$. O carregamento $W$, aplicado uniformemente na aresta direita, é igual a 10. As propriedades de interesse do material apresentam os seguintes valores: $E=1000$ (módulo de Young) e $v=0,3$ (coeficiente de Poisson). O centro do orifício encontra- 
se eqüidistante das arestas laterais e a uma distância e da aresta inferior ou $c$ da aresta superior. Neste problema considerou-se uma relação fixa $c / e=2$ e adotou-se $e=a=20$ e espessura unitária. Além disso, em função das análises que foram realizadas, atribuiu-se ao raio do orifício alguns valores de interesse, tal que $0,1 \leq r / e \leq 0,5$.

Na obtenção dos valores de referência realizou-se uma análise via o MEF clássico com o auxílio do software ANSYS $^{\circledR}$. A discretização adotada envolveu 1278 elementos distribuídos mediante um refinamento prioritário em algumas regiões importantes do domínio: no entorno à região do orifício circular, na aresta direita em virtude do carregamento aplicado e na esquerda onde a chapa está vinculada, conforme ilustrado na Figura (7.29). O elemento adotado foi o mesmo das análises realizadas nos problemas anteriores. Desta forma, 2714 nós foram utilizados e consequentemente, um total de 5428 graus de liberdade foi despendido.

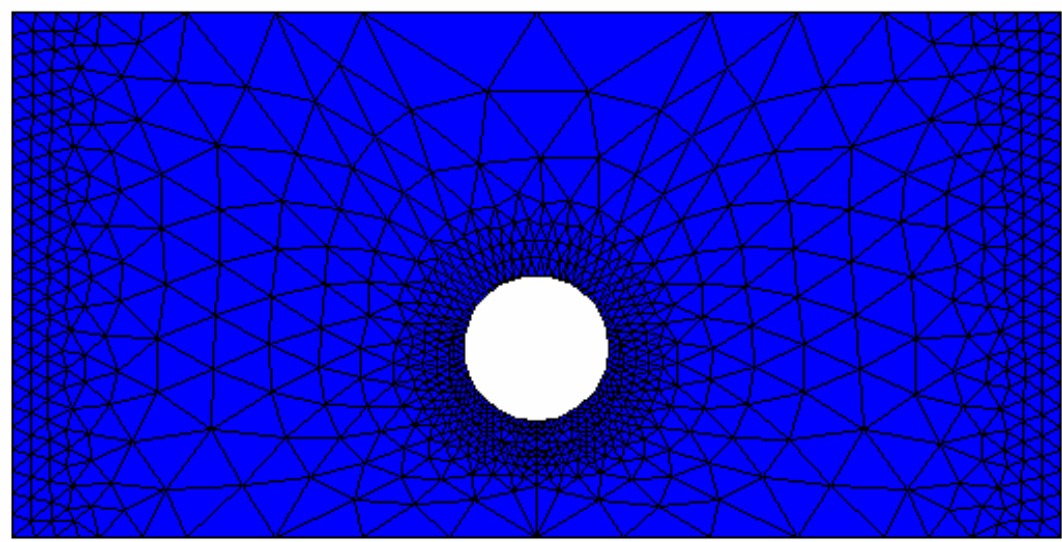

Figura 7.29. Discretização adotada na análise realizada via o MEF clássico para obtenção dos valores de referência do $3^{\circ}$ problema.

Para a energia de deformação obteve-se $U_{R E F}=417,16$, enquanto, para o deslocamento de referência encontraram-se os seguintes valores: $u_{\text {XREF }}=1,583$ e $u_{\text {YREF }}=0,4784$, referentes ao ponto do canto inferior direito. Por sua vez a distribuição de tensões obtida está indicada na Figura (7.30).

Neste problema duas modalidades de refinamento foram exploradas. Inicialmente realizaram-se análises de convergência de algumas grandezas de interesse mediante o refino-p das três bases exploradas neste trabalho: $\beta_{1}, \beta_{2}$ e $\beta_{3}$. Em seguida avaliaram-se algumas situações de enriquecimento seletivo, o qual abrangeu determinados elementos localizados no entorno do orifício da discretização adotada. 


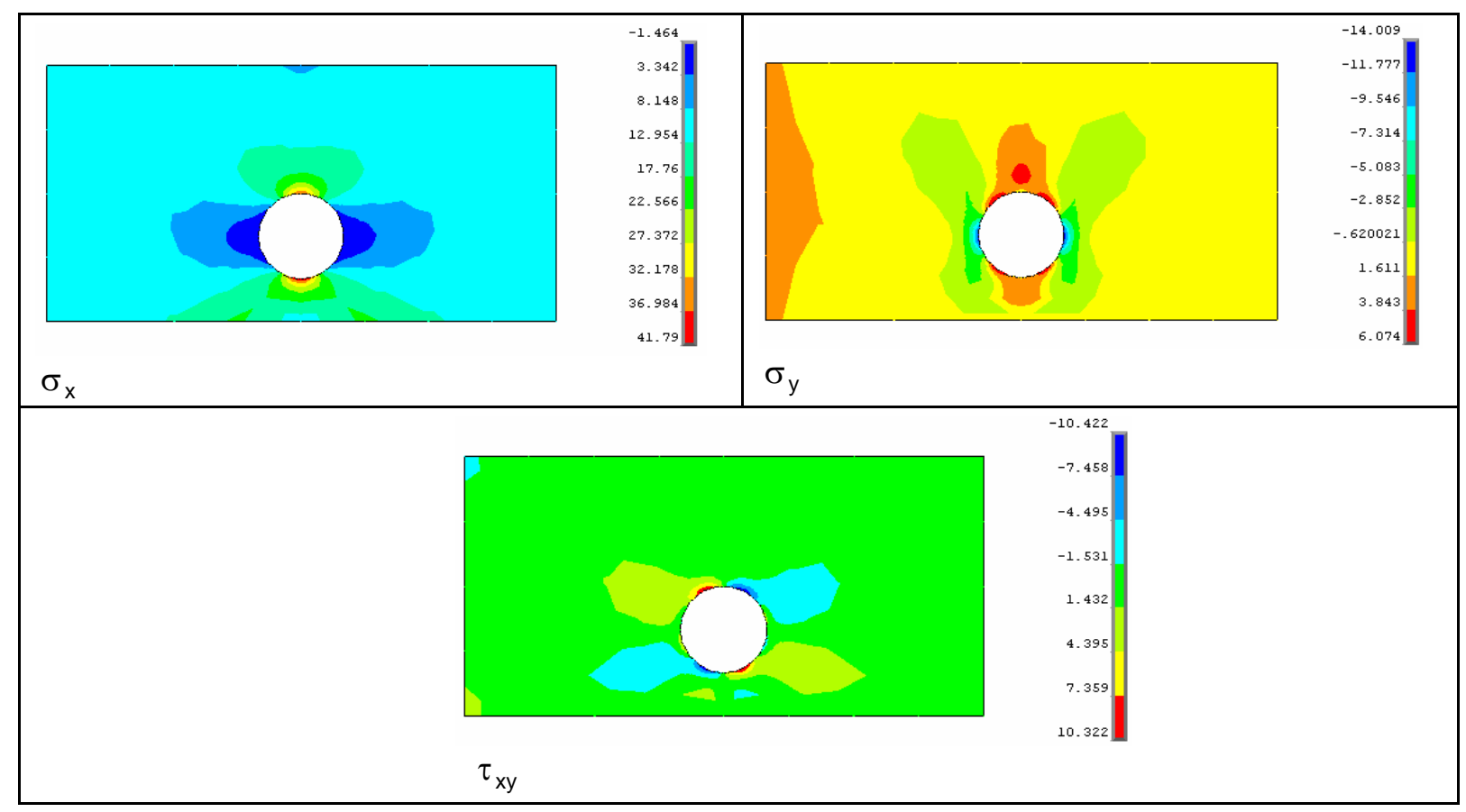

Figura 7.30. Distribuição de tensões de referência do $3^{\circ}$ problema.

- Refino-p

No refino-p adotou-se uma discretização composta de seis elementos, sendo que um deles envolve o orifício, conforme ilustrado na Figura (7.31).

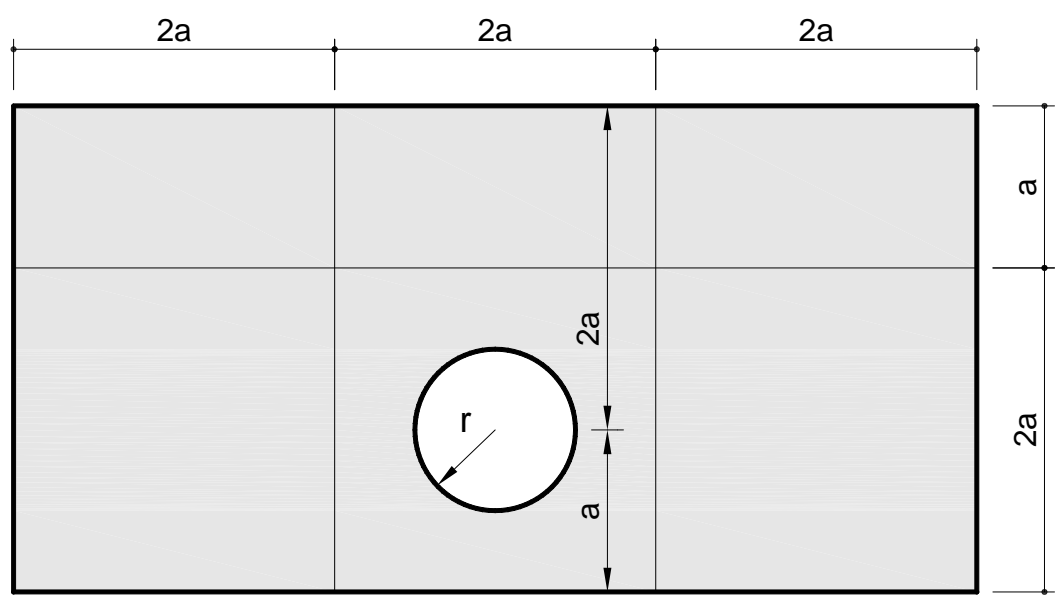

Rede irregular $3 \times 2$

Figura 7.31. Discretização adotada no refino-p do $3^{\circ}$ problema.

O enriquecimento incidiu sobre todos os elementos da discretização adotada e sobre algumas fronteiras estáticas, como indicado na Figura (7.32). Nela ainda ilustram-se os pontos adotados nas aproximações de algumas grandezas de interesse, a saber, quina inferior para o deslocamento horizontal e o ponto inferior do orifício, onde, segundo Peterson (1974), a tensão normal é máxima. 


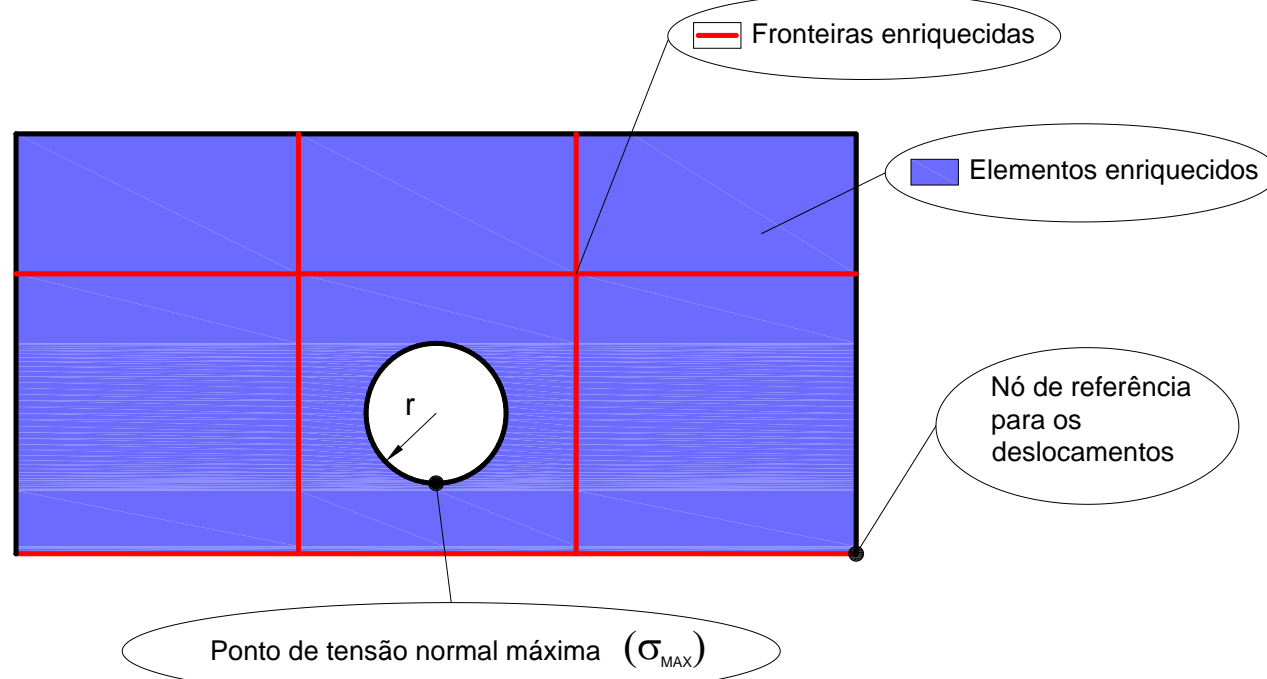

Figura 7.32. Elementos e fronteiras estáticas enriquecidos no refino-p.

Nas análises de convergência adotou-se $r=10$ estabelecendo-se a seguinte relação entre o raio e a excentricidade: $r / e=0,5$. Desta forma, para a energia de deformação obtevese a curva que quantifica o erro relativo indicada na Figura (7.33).

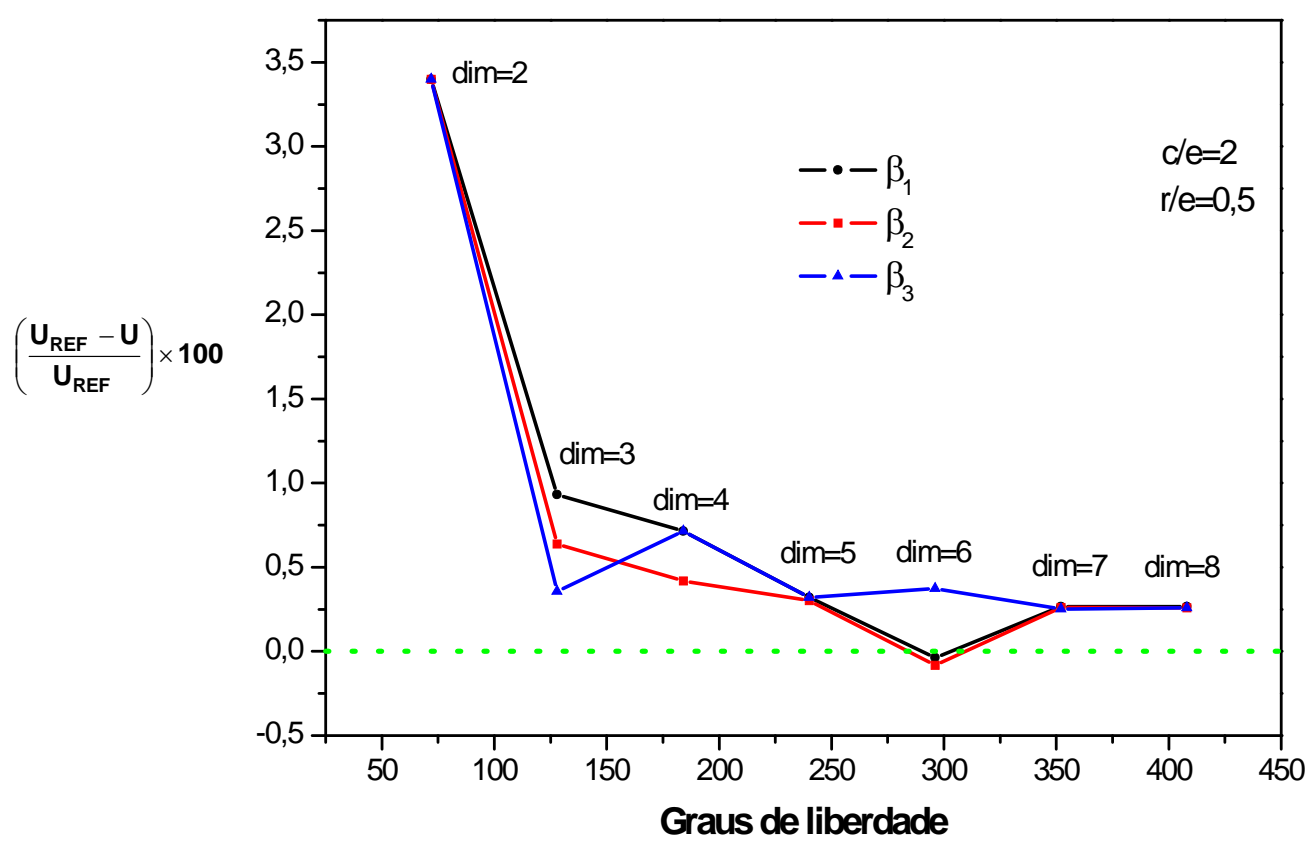

Figura 7.33. Refino-p. Erro relativo da energia de deformação (\%).

Para o deslocamento horizontal do ponto de referência encontrou-se a curva com o aspecto indicado na Figura (7.34). 


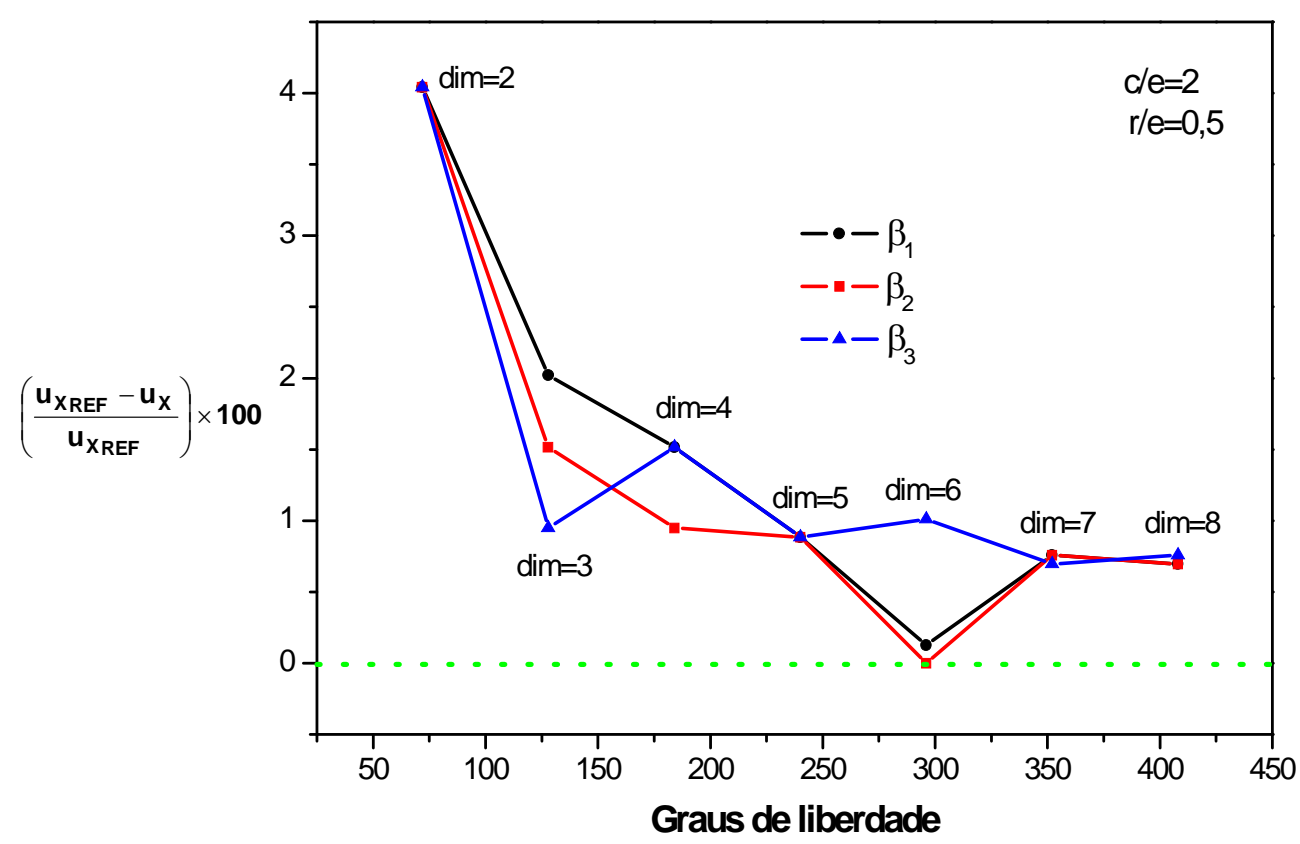

Figura 7.34. Refino-p. Erro relativo do deslocamento horizontal de referência (\%).

Por sua vez a curva obtida que quantifica o erro relativo do fator de concentração de tensões pode ser visualizada na Figura (7.35).

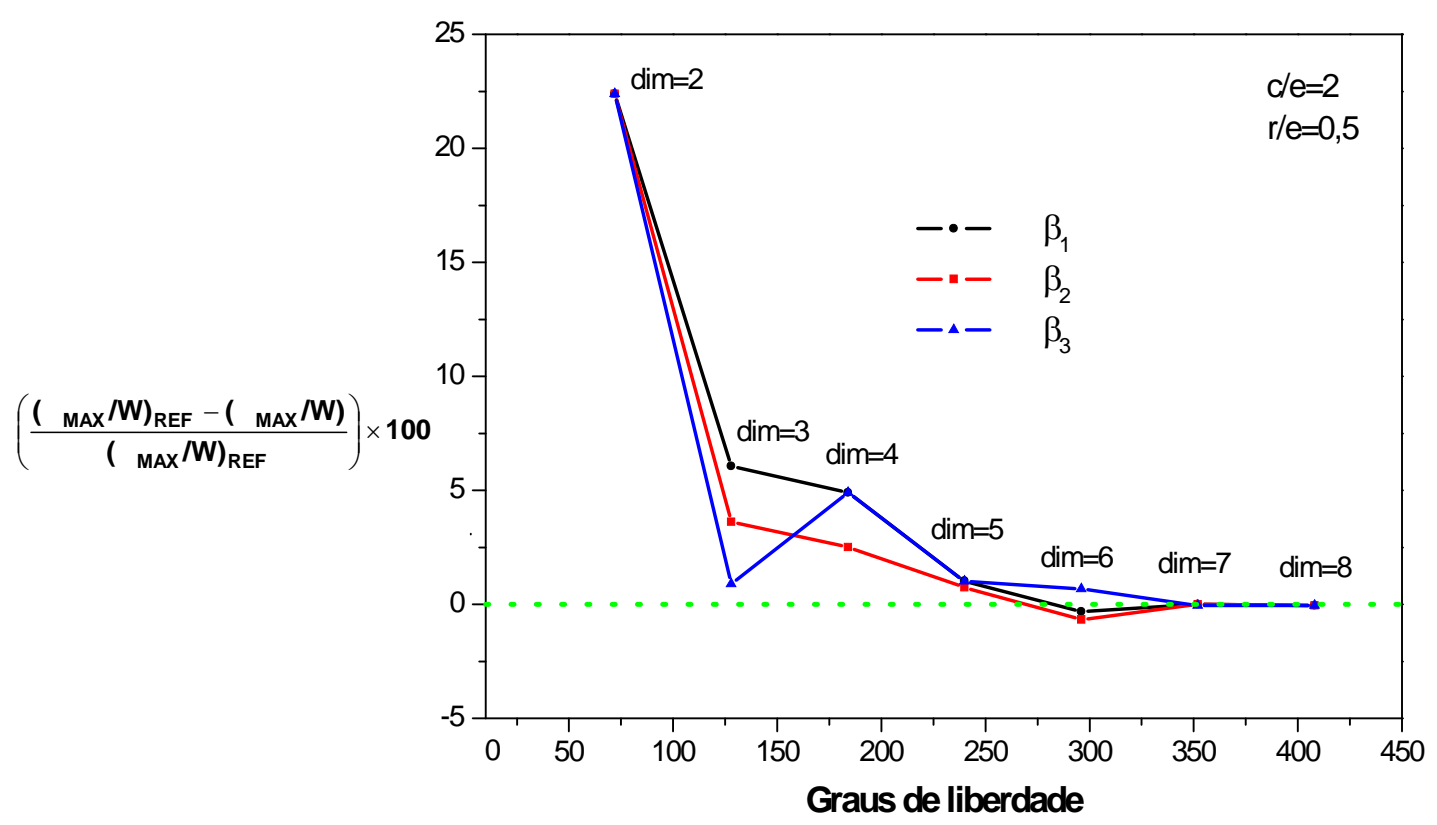

Figura 7.35. Refino-p. Erro relativo do fator de concentração de tensões (\%).

$\mathrm{Na}$ Figura (7.35) o valor de referência adotado corresponde à solução exata do problema: $\left(\sigma_{\text {MAX }} / W\right)_{\text {REF }}=4,140$, sendo $W$ o carregamento na aresta direita (PETERSON, 1974).

Verifica-se das Figuras (7.33), (7.34) e (7.35) que, apesar do reduzido número de 
graus de liberdade envolvidos (cerca de um doze avos dos utilizados na análise via o MEF clássico), as curvas construídas convergiram para os valores de referência, particularmente com destaque para o fator de concentração de tensões, que praticamente coincidiu com o valor exato (erro de $0,04 \%$ ). Novamente, verifica-se que não houve divergência apreciável entre as três bases utilizadas.

No tocante à distribuição de tensões também se obteve uma excelente qualidade de resposta para as três bases utilizadas. Em particular, para a base $\beta_{1}$ com $\operatorname{dim}=7$, situação que envolveu 352 graus de liberdade, tem-se a representação indicada na Figura (7.36).

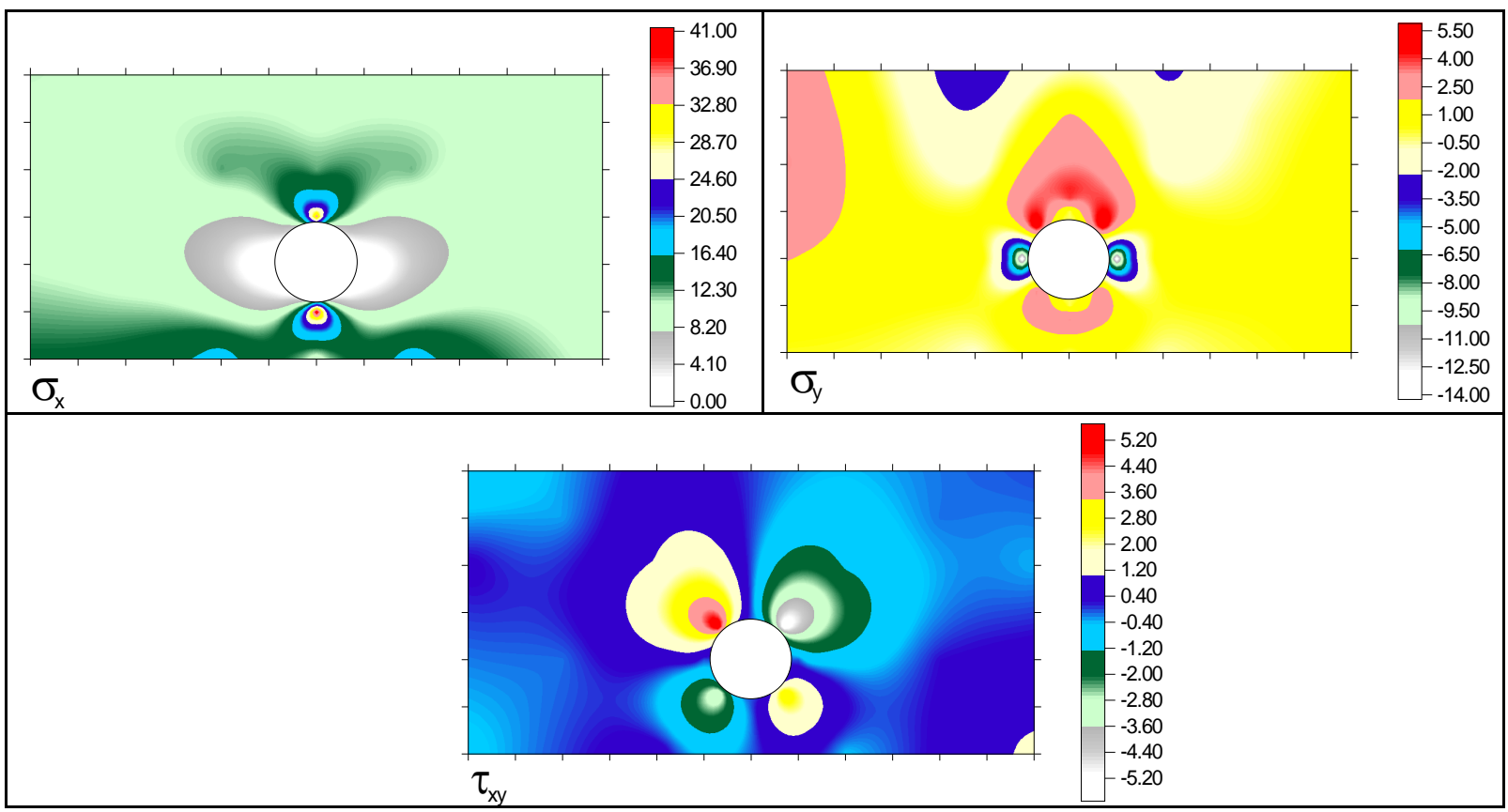

Figura 7.36. Distribuição de tensões obtida no refino-p com a base $\beta_{1}$ com $\operatorname{dim}=7$.

Uma última situação concernente ao refino-p foi averiguada. Nela manteve-se a aproximação das três bases com dimensão igual a sete, situação que envolveu 352 graus de liberdade, e variou-se o raio do orifício, tal que $0,1 \leq r / e \leq 0,5$. Os valores obtidos para cada relação de $r / e$ foram confrontados com os valores exatos, os quais foram extraídos de Peterson (1974). Os resultados obtidos se aproximaram notavelmente dos valores exatos, conforme ilustrado na Figura (7.37). Contudo, verificou-se uma relação de proporcionalidade inversa entre os valores de $r / e$ com a norma do resíduo do erro do sistema resolvente. Para $r / e=0,5$ obteve-se uma norma em torno de $1 \times 10^{-10}$ enquanto para $r / e=0,1$ este valor aumentou para $1 \times 10^{-5}$. Este fato se deve a perda de precisão numérica em virtude das elevadas ordens de potência nas operações algébricas que envolvem o raio do orifício (vide os vetores de aproximação no apêndice B). Tal fenômeno pode oportunamente ser contornado, 
adotando-se elementos com orifício com dimensões não muito superiores à do raio correspondente.

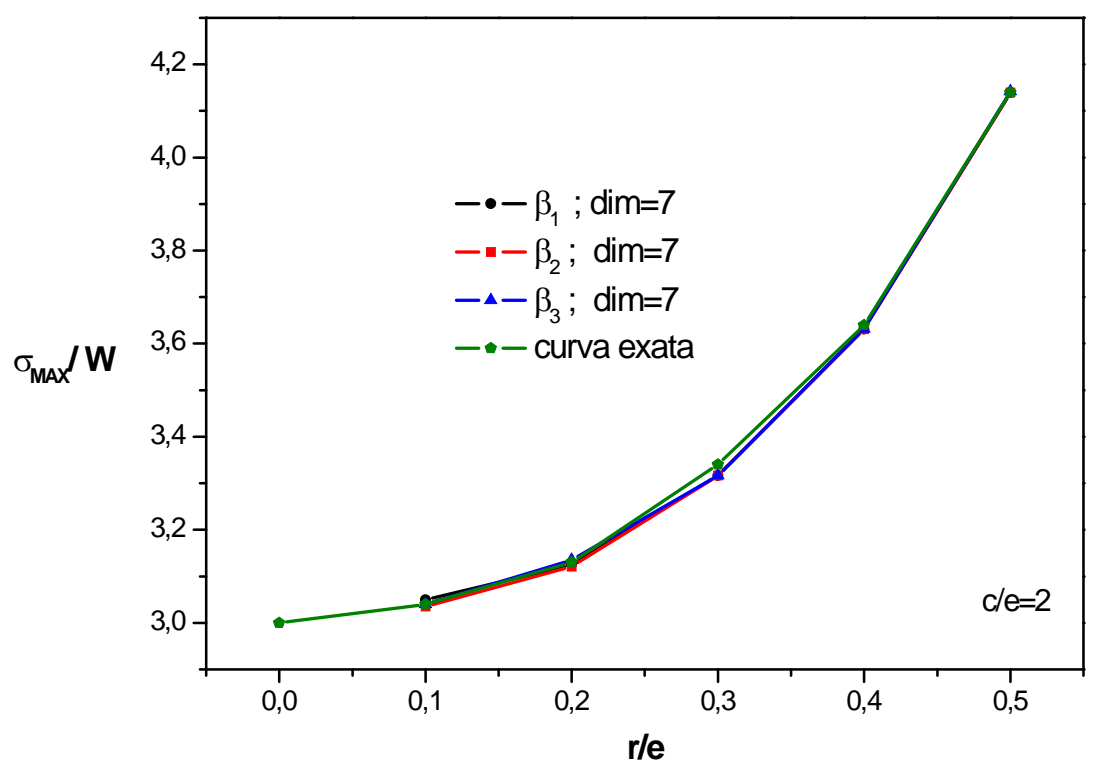

Figura 7.37. Fator de concentração de tensões para diferentes relações de $r / e$.

Salienta-se que o teste do "mosaico" foi satisfeito em todas as análises descritas anteriormente.

- .Enriquecimento seletivo

No enriquecimento seletivo analisaram-se algumas situações de enriquecimento com as três bases exploradas neste trabalho: $\beta_{1}, \beta_{2}$ e $\beta_{3}$. A discretização adotada aproxima a geometria do furo do orifício por 16 segmentos de reta, conforme ilustrado na Figura (7.38).

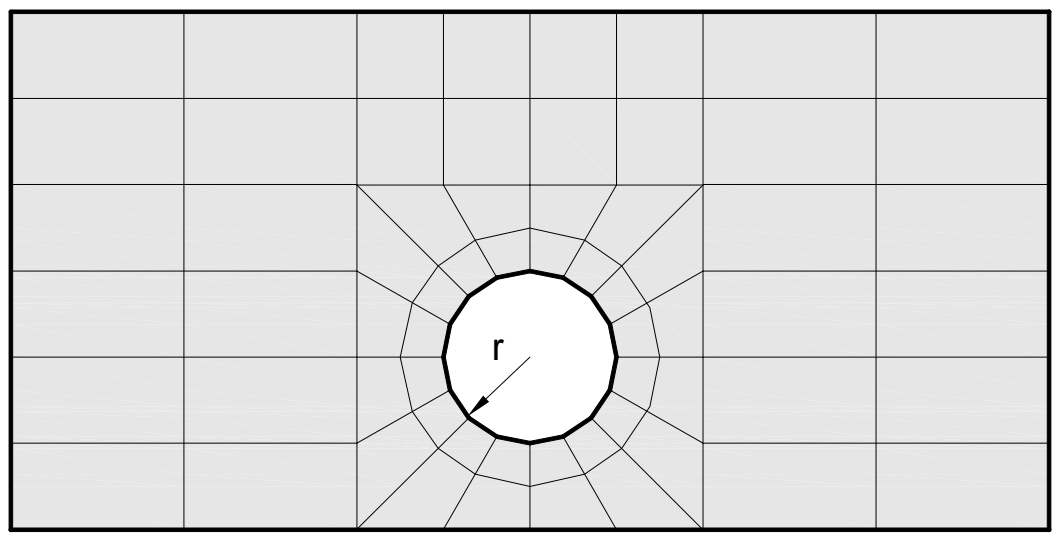

Figura 7.38. Discretização adotado no enriquecimento seletivo.

Com o intuito de melhorar a distribuição de tensões no entorno do orifício, 
enriqueceu-se a região indicada na Figura (7.39). Tal região abrangeu 32 elementos e 64 fronteiras estáticas.

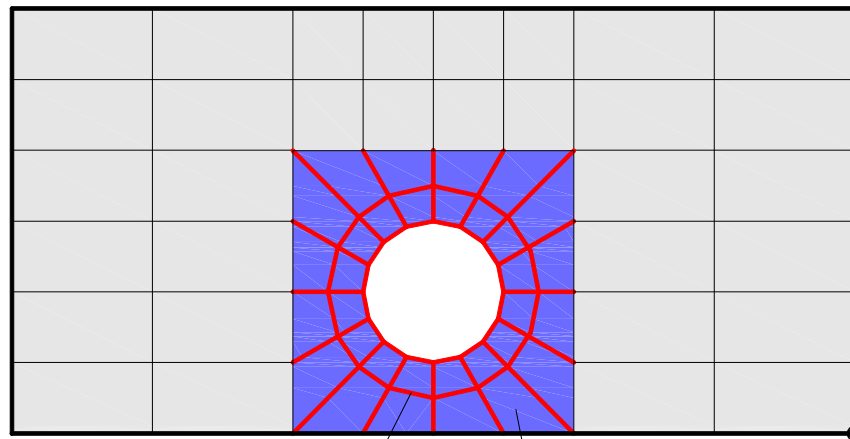

Nó de referência

para os

deslocamentos

Fronteiras enriquecidas

Elementos enriquecidos

Figura 7.39. Elementos e fronteiras enriquecidas no enriquecimento seletivo.

Os resultados para as situações de enriquecimento que foram consideradas estão apresentados na Tabela (7.9), enquanto os graus de liberdade envolvidos estão indicados na Tabela (7.10). Ressalta-se que em todas as situações avaliadas o teste do "mosaico" foi uma condição preestabelecida.

Tabela 7.9 - Grandezas de interesse aproximadas para algumas situações de enriquecimento, quando se enriqueceu 32 elementos e 64 fronteiras estáticas

\begin{tabular}{|c|c|c|c|c|c|c|c|c|}
\hline \multicolumn{3}{|c|}{ Condições de Enriquecimento } & \multicolumn{6}{|c|}{ Grandezas Aproximadas } \\
\hline \multirow{3}{*}{$\begin{array}{c}\text { Graus } \\
\text { de liberdade } \\
\text { em tensão no } \\
\Omega_{\mathrm{e}} \text { da } \\
\text { região } \\
\text { enriquecida }\end{array}$} & \multirow{2}{*}{\multicolumn{2}{|c|}{$\begin{array}{c}\text { Base em } \Gamma_{\text {te }} \text { da } \\
\text { região } \\
\text { enriquecida }\end{array}$}} & \multirow{2}{*}{\multicolumn{2}{|c|}{$\begin{array}{c}\begin{array}{c}\text { Energia } \\
\text { de } \\
\text { deformação }\end{array} \\
U_{R E F}=417,16\end{array}$}} & \multicolumn{4}{|c|}{$\begin{array}{c}\text { Deslocamentos no ponto de } \\
\text { referência }\end{array}$} \\
\hline & & & & & \multicolumn{2}{|c|}{$\begin{array}{c}\mathbf{E m} \mathbf{x} \\
u_{X R E F}=1,583\end{array}$} & \multicolumn{2}{|c|}{$\begin{array}{c}\text { Em y } \\
u_{Y R E F}=0,4784\end{array}$} \\
\hline & Tipo & Dimensão & $\begin{array}{l}\text { Valor } \\
\text { obtido }\end{array}$ & $\begin{array}{c}\text { Erro } \\
\text { relativo } \\
(\%)\end{array}$ & $\begin{array}{l}\text { Valor } \\
\text { obtido }\end{array}$ & $\begin{array}{c}\text { Erro } \\
\text { relativo } \\
(\%)\end{array}$ & $\begin{array}{l}\text { Valor } \\
\text { obtido }\end{array}$ & $\begin{array}{c}\text { Erro } \\
\text { relativo } \\
(\%)\end{array}$ \\
\hline 9 & $\beta_{0}$ & 2 & 409,46 & 1,84 & 1,537 & 2,90 & 0,4250 & 11,16 \\
\hline 43 & $\beta_{1}$ & 7 & 413,23 & 0,942 & 1,564 & 1,20 & 0,4537 & 5,16 \\
\hline 43 & $\beta_{2}$ & 7 & 413,23 & 0,942 & 1,564 & 1,20 & 0,4536 & 5,18 \\
\hline 43 & $\beta_{3}$ & 7 & 413,22 & 0,944 & 1,564 & 1,20 & 0,4535 & 5,20 \\
\hline
\end{tabular}

Tabela 7.10 - Graus de liberdade envolvidos para algumas situações de enriquecimento, quando se enriqueceu 32 elementos e 64 fronteiras estáticas

\begin{tabular}{|c|c|c|c|c|}
\hline \multicolumn{2}{|c|}{ Graus de liberdade em $\Omega_{\mathrm{e}}$ e base utilizada } & \multicolumn{2}{|c|}{ Dimensão da base em $\Gamma_{\text {te }}$} & \multirow{2}{*}{$\begin{array}{c}\text { Total de } \\
\text { graus de } \\
\text { liberdade }\end{array}$} \\
\hline $\begin{array}{c}\text { Na região não } \\
\text { enriquecida }\end{array}$ & $\begin{array}{c}\text { Na região } \\
\text { enriquecida }\end{array}$ & $\begin{array}{c}\text { Na região não } \\
\text { enriquecida }\end{array}$ & $\begin{array}{c}\text { Na região } \\
\text { enriquecida }\end{array}$ & \\
\hline $9-\beta_{0}$ & $9-\beta_{0}$ & 2 & 2 & 734 \\
\hline $9-\beta_{0}$ & $43-\beta_{1}, \beta_{2}$ ou $\beta_{3}$ & 2 & 7 & 2462 \\
\hline
\end{tabular}


De acordo com a Tabela (7.9) o enriquecimento promoveu melhorias nas grandezas de interesse aproximadas, as quais apresentaram valores muito próximos dos valores de referência. Novamente, a eficiência das três bases utilizadas foi confirmada perante a proximidade dos resultados obtidos nas situações avaliadas.

A tendência positiva do enriquecimento foi evidenciada, sobretudo, na distribuição de tensões. Os resultados obtidos apontaram eqüidade entre as três bases exploradas e uma distribuição de tensões coerente com os resultados anteriores (refino-p com o uso do elemento com orifício e análise via o MEF clássico). Por exemplo, para a base $\beta_{1} \operatorname{com} \operatorname{dim}=7$, situação que envolveu 2462 graus de liberdade (inferior à metade dos utilizados na análise via o MEF clássico), o confronto dos resultados obtidos com ou sem o enriquecimento seletivo pode ser visualizado na Figura (7.40).

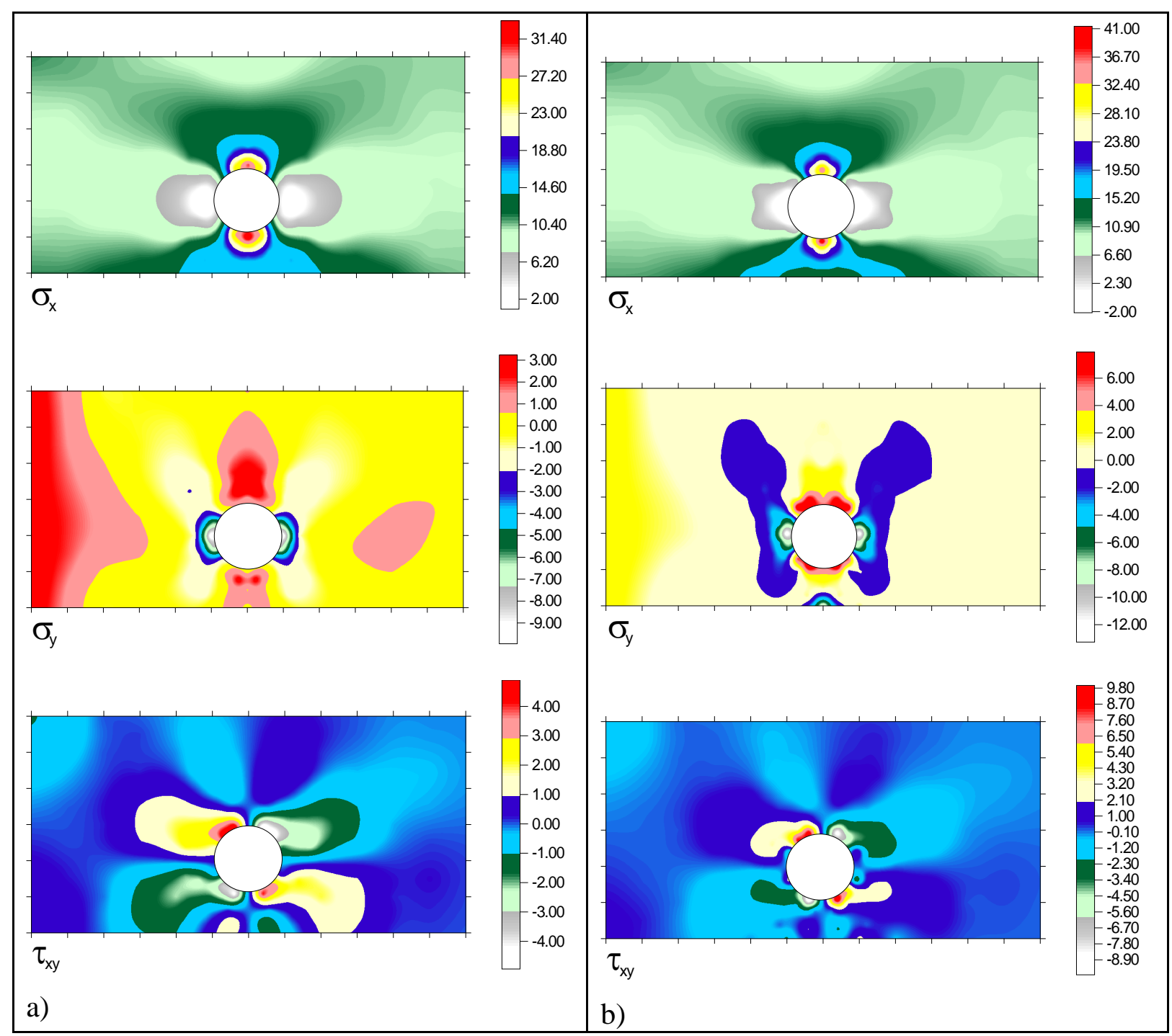

Figura 7.40. Distribuição de tensões. a) Situação não enriquecida e b) Enriquecimento com a base $\beta_{1}$ com $\operatorname{dim}=7$. 


\section{5 $4^{\circ}$ Problema: chapa com fissura inserida no domínio}

O quarto problema trata de uma chapa com uma fissura inserida simetricamente em seu domínio. A chapa encontra-se submetida a um carregamento auto-equilibrado $(W=1)$, conforme ilustrado na Figura (7.41).

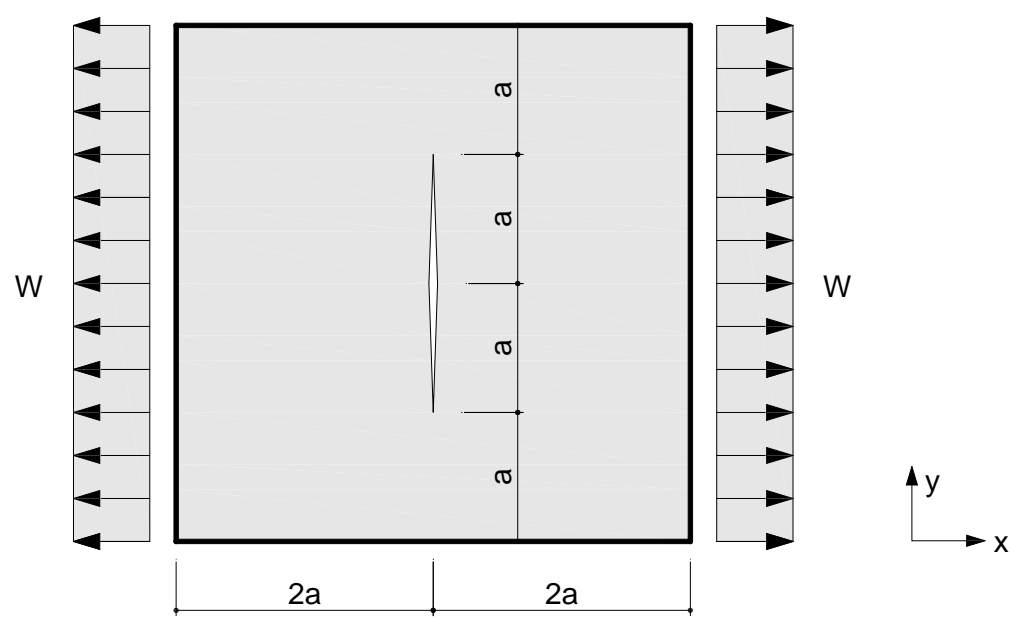

Figura 7.41. Chapa analisada no $4^{\circ}$ problema. Sua geometria e carregamento.

No dimensionamento da referida chapa adotou-se $a=0,5$ e espessura unitária. As propriedades de interesse do material, módulo de Young e coeficiente de Poisson, apresentam, respectivamente, os seguintes valores: $E=1,0$ e $v=0,3$.

A solução deste problema foi obtida segundo o Método da Partição em formulação híbrida-Trefftz com enriquecimento seletivo, apresentada no capítulo 5. Inicialmente realizouse uma análise de convergência do fator de intensidade de tensões $K_{\mathrm{I}}$, o qual foi extraído mediante a técnica de correlação dos deslocamentos, relatada no item 5.4. Em seguida, obteve-se a distribuição da componente de tensão $\boldsymbol{\sigma}_{\boldsymbol{x}}$. Em ambas as avaliações, se averiguaram a influência do refino-p e da série de carregamentos fictícios na resposta obtida.

Ressalta-se que nas análises realizadas simplificou-se a aplicação do referido método adotando-se o caminho interno $\Gamma_{\mathrm{f}}$ como sendo o próprio contorno do volume de influência local da fissura $\boldsymbol{V}_{\boldsymbol{f}}$. Neste sentido, $\Gamma_{\mathrm{f}}$ foi completamente impedido de deslocar-se, propiciando apenas o cálculo de tensões em $\Gamma_{\mathrm{f}}$, ao invés de tensões e deslocamentos.

Nas análises dos problemas globais $P G_{0}$ e $P G_{k}$ adotou-se a discretização indicada na Figura (7.42 a). Nos problemas locais $P \boldsymbol{L}_{\boldsymbol{k}}$ manteve-se o mesmo refinamento dos problemas globais no entorno à região da fissura, conforme indicado na Figura (7.42 b). Nela, ainda, 
ilustram-se a vinculação considerada, a série de carregamentos fictícios e o ponto adotado para o cálculo de $K_{\mathrm{I}}$. Na Figura $(7.42 \mathrm{c}$ ) indicam-se os significados das linhas coloridas evidenciadas nas Figuras (7.42 a) e (7.42 b).

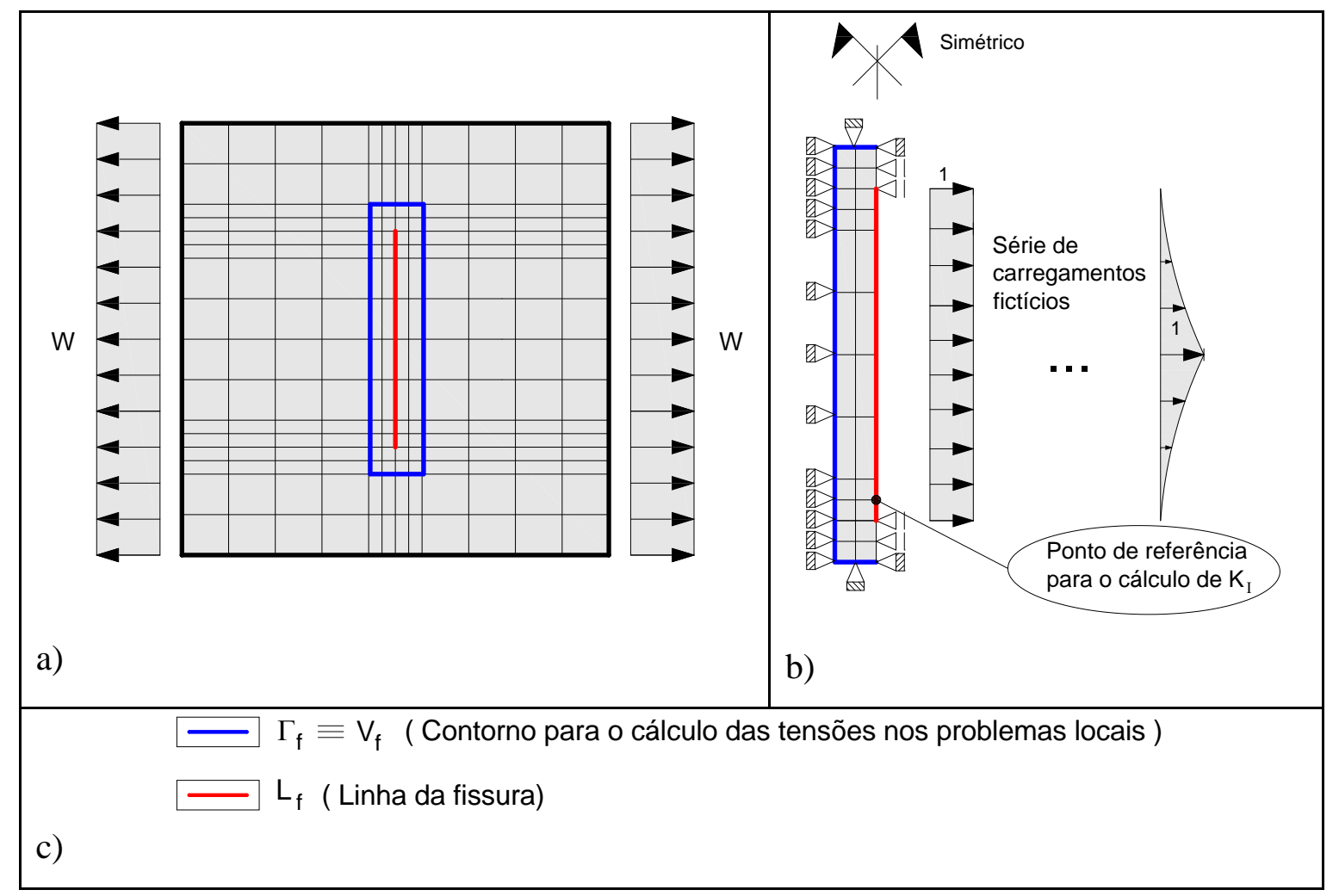

Figura 7.42. a) Discretização adotada em $P G_{0}$ e nos $P G_{k}$, b) Discretização adotada nos $P L_{k}$ e outros aspectos de interesse e c) Legenda das linhas coloridas.

Conforme mencionado no capítulo 5 o Método da Partição consiste em analisar um determinado PVC mediante a sobreposição adequada de uma série de subproblemas globais e locais. Desta forma, as modalidades de refinamento, seja o refino-h, o refino-p ou o enriquecimento seletivo podem incidir sobre cada um dos subproblemas concebidos.

Neste problema, em particular, considerou-se o refino-p apenas nos problemas locais, de forma que a região enriquecida abrangeu todos os elementos e fronteiras estáticas da discretização indicada na Figura $\left(7.42\right.$ b). No referido refino utilizou-se a base $\beta_{1}$ para a aproximação dos deslocamentos nas fronteiras estáticas, sempre atendendo ao teste do "mosaico".

Assim sendo, a partir da expansão da série de carregamentos fictícios obteve-se a curva que quantifica o erro relativo do fator de intensidade de tensões normalizado $\tilde{K}_{\mathrm{I}}$, para diferentes situações de enriquecimento da base $\beta_{1}$, conforme ilustrado na Figura (7.43). 


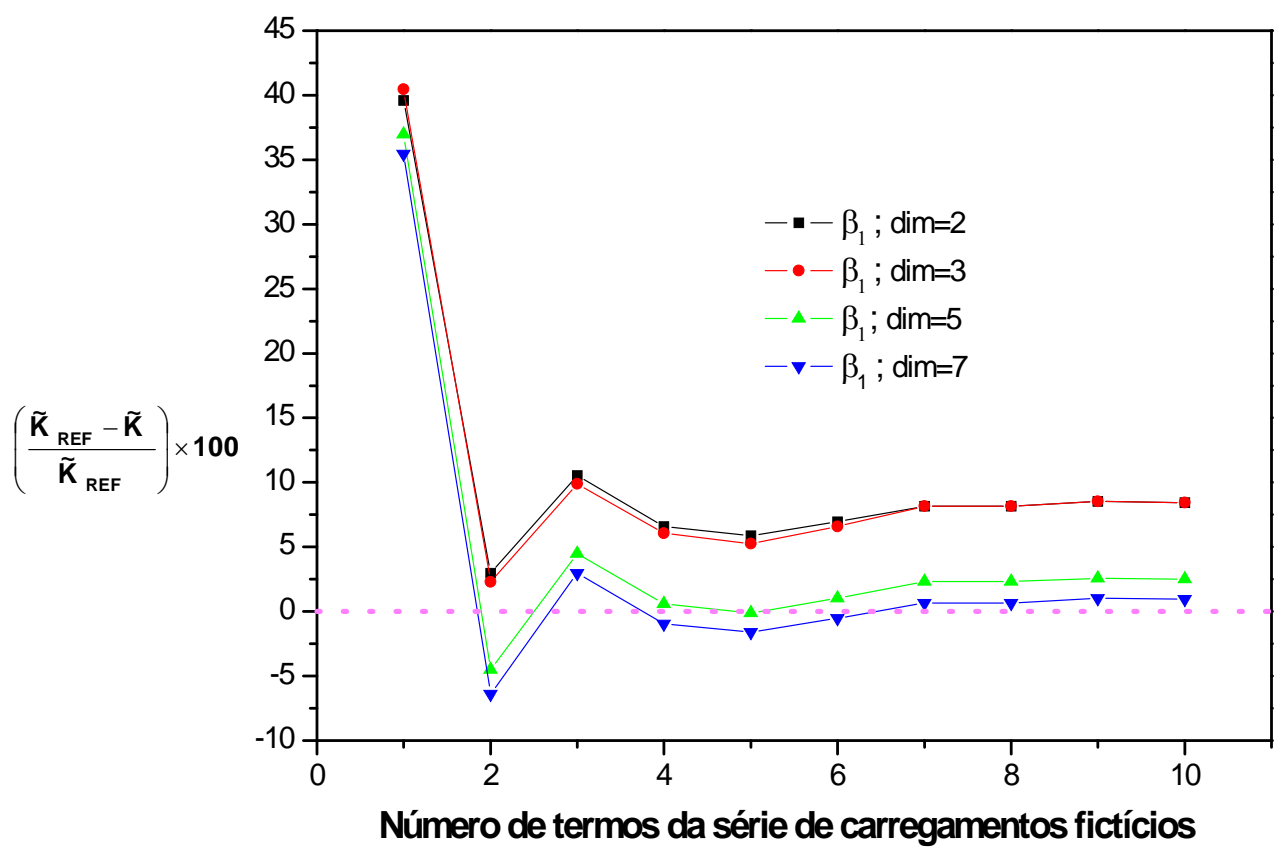

Figura 7.43. Erro relativo do fator de intensidade de tensões (\%).

O valor de referência adotado para o fator de intensidade de tensões foi extraído do trabalho de Pereira (2004). Tal valor corresponde ao fator de intensidade de tensões normalizado: $\tilde{K}_{\mathrm{I}}$ ref $=K_{\mathrm{I}} / \sqrt{\pi a}=1,3341$.

Na Figura (7.43), observa-se que para o primeiro termo da série de carregamentos fictícios o valor aproximado de $\tilde{K}_{\mathrm{I}}$ afastou-se muito do valor de referência, mesmo para um elevado nível de refino-p. Por outro lado, para o segundo termo da série de carregamentos, verificou-se uma mudança brusca no valor aproximado de $\tilde{K}_{\mathrm{I}}$, o qual se aproximou razoavelmente do valor de referência, mas a contribuição do refino-p ainda não pode ser evidenciada. A partir do terceiro termo da série, as curvas mantiveram-se sem alterações significativas, para cada situação analisada; mas o refino-p foi fundamental para garantir a convergência dos valores aproximados de $\tilde{K}_{\mathrm{I}}$ para o valor de referência. No refino da base $\beta_{1}$ com $\operatorname{dim}=7$ o erro relativo foi de $0,95 \%$.

Por sua vez a distribuição obtida de tensões $\sigma_{\boldsymbol{x}}$ pode ser visualizada nas Figuras (7.44) e (7.45). Na Figura (7.44 a) está ilustrado a distribuição para o primeiro termo da série de carregamentos fictícios, quando se considerou a base $\beta_{1}$ sem enriquecimento, enquanto na Figura (7.44 b) representa-se a distribuição, ainda para o primeiro termo da série, mas quando se utilizou a base $\beta_{1}$ com dimensão igual a sete. Analogamente, para o décimo termo da série de carregamentos fictícios, as distribuições obtidas nas situações analisadas com ou sem o enriquecimento da base $\beta_{1}$ estão, respectivamente, ilustradas nas Figuras (7.45 a) e (7.45 b). 


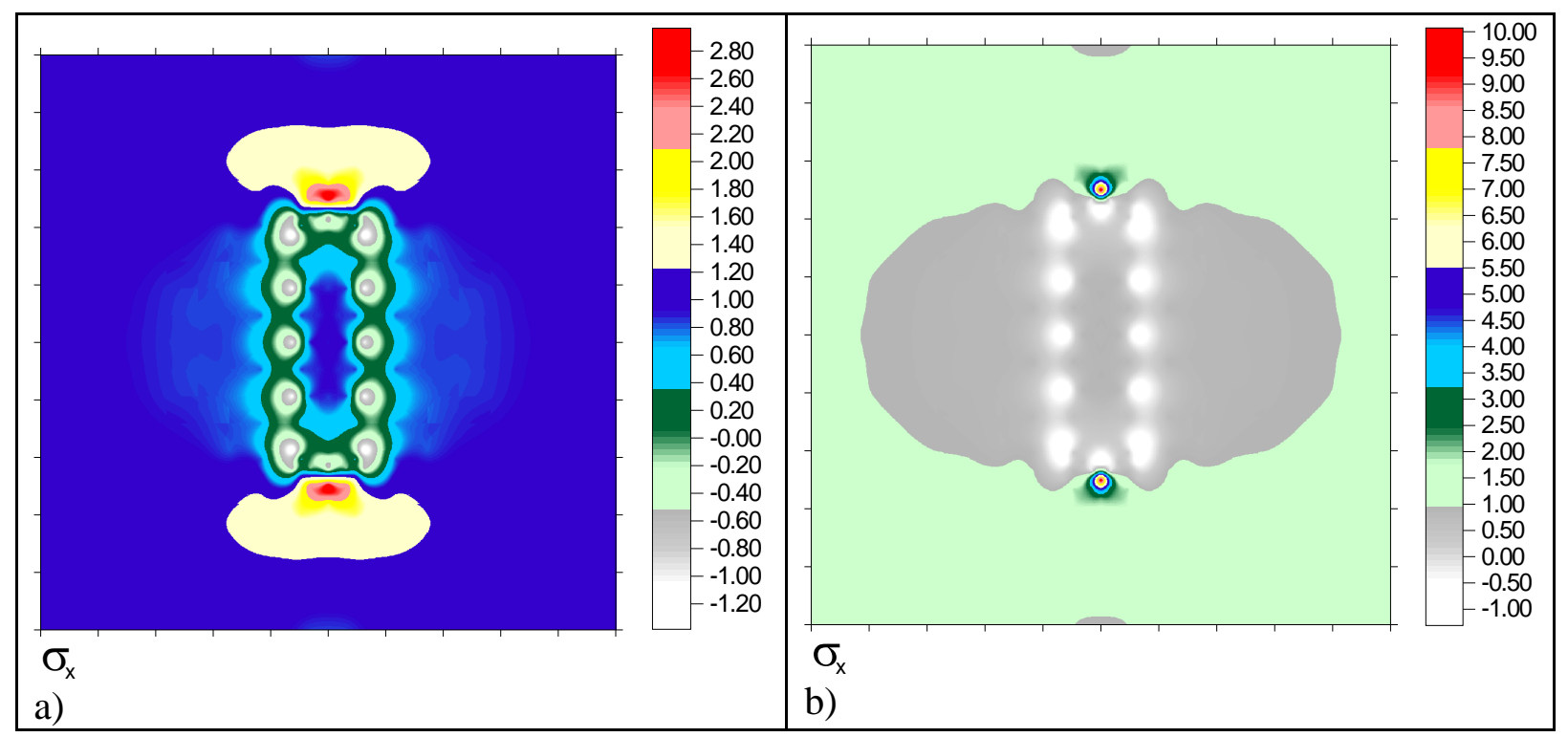

Figura 7.44. a) Distribuição de tensões para o $1^{\circ}$ termo da série de carregamentos fictícios, quando se utilizou a base $\beta_{1}$ sem enriquecimento e b) Distribuição de tensões para o $1^{\circ}$ termo da série de carregamentos fictícios, quando se utilizou a base $\beta_{1}$ com $\operatorname{dim}=7$.

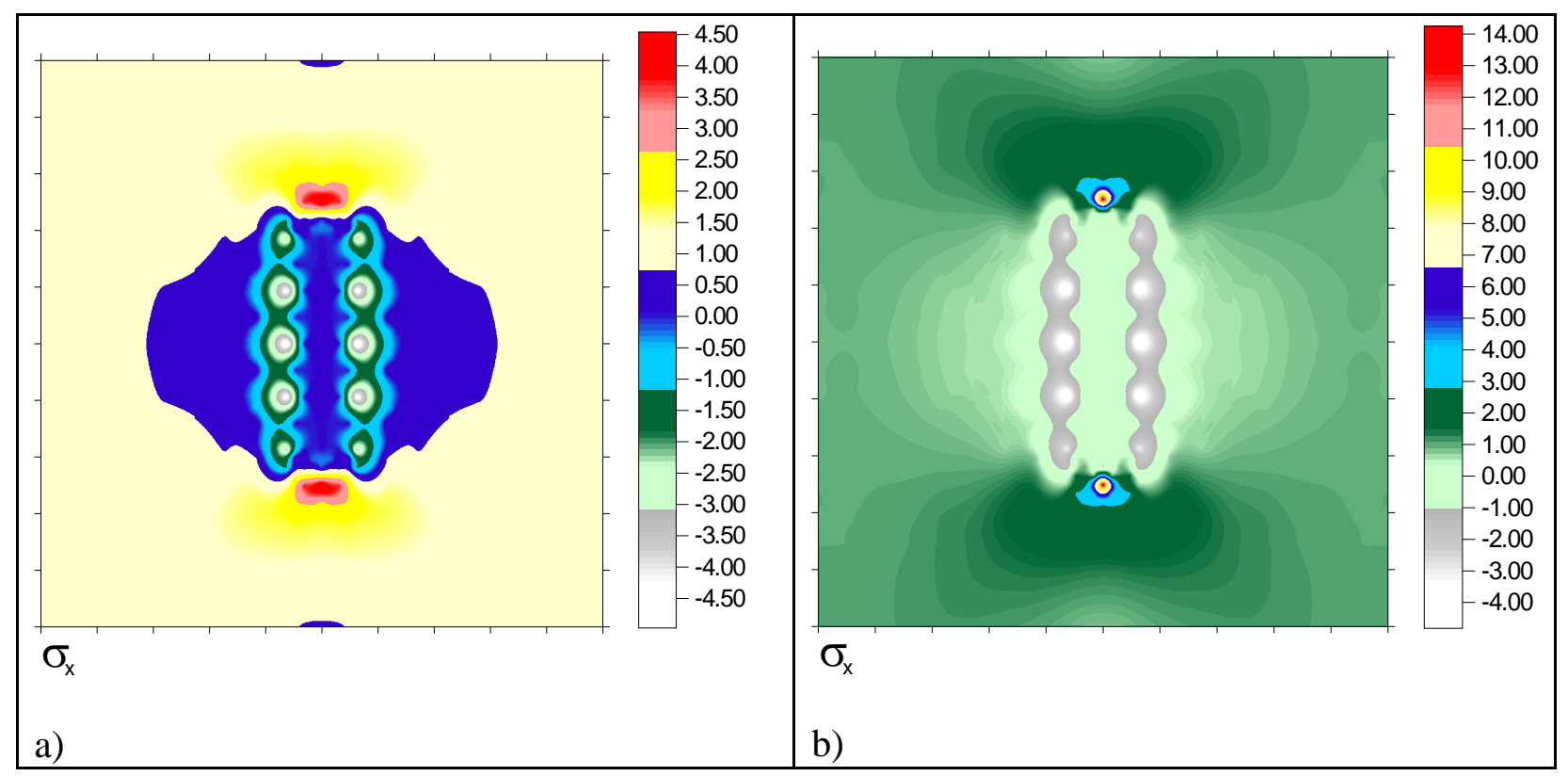

Figura 7.45. a) Distribuição de tensões para o $10^{\circ}$ termo da série de carregamentos fictícios, quando se utilizou a base $\beta_{1}$ sem enriquecimento e b) Distribuição de tensões para o $10^{\circ}$ termo da série de carregamentos fictícios, quando se utilizou a base $\beta_{1}$ com $\operatorname{dim}=7$.

Percebe-se na Figura (7.44) que o refino-p, ainda que aplicado somente nos problemas locais, promoveu um aumento das estimativas de tensões $\sigma_{\boldsymbol{x}}$ no entorno da ponta da fissura. O mesmo fato pode ser verificado na Figura (7.45), mas com valores relativamente superiores, atestando a influência positiva tanto do refino-p quanto da expansão da série de 
carregamentos fictícios. Contudo, em todas as situações averiguadas, surgiram zonas que apresentaram valores incorretos de tensões. Atentamente, percebe-se que tais zonas correspondem à região de vinculação dos problemas locais, isto é, ao $\Gamma_{f}$ o qual foi adotado como sendo o próprio contorno de $\boldsymbol{V}_{\boldsymbol{f}}$. Infere-se que tal fenômeno pode ser atenuado considerando-se um $\Gamma_{\mathrm{f}}$ distinto do contorno de $\boldsymbol{V}_{\boldsymbol{f}}$ e adotando-se a menor quantidade possível de vínculos em $\boldsymbol{V}_{\boldsymbol{f}}$. Neste contexto, motivam-se novos estudos para contornar este inconveniente, seja de forma atenuada como se inferiu ou por considerações extras que traduzem a continuidade, em forma fraca, de tensões ao longo do contorno de $\boldsymbol{V}_{\boldsymbol{f}}$.

\section{6 $5^{\circ}$ Problema: painel de Cook}

O último problema refere-se a uma chapa impedida de deslocar-se na aresta esquerda segundo as direções $x$ e $y\left(u_{x}=u_{y}=0\right)$ e submetida a um carregamento uniformemente distribuído ( $W=0,0625)$, segundo a direção $y$, em sua aresta direita, conforme ilustrado na Figura (7.46).

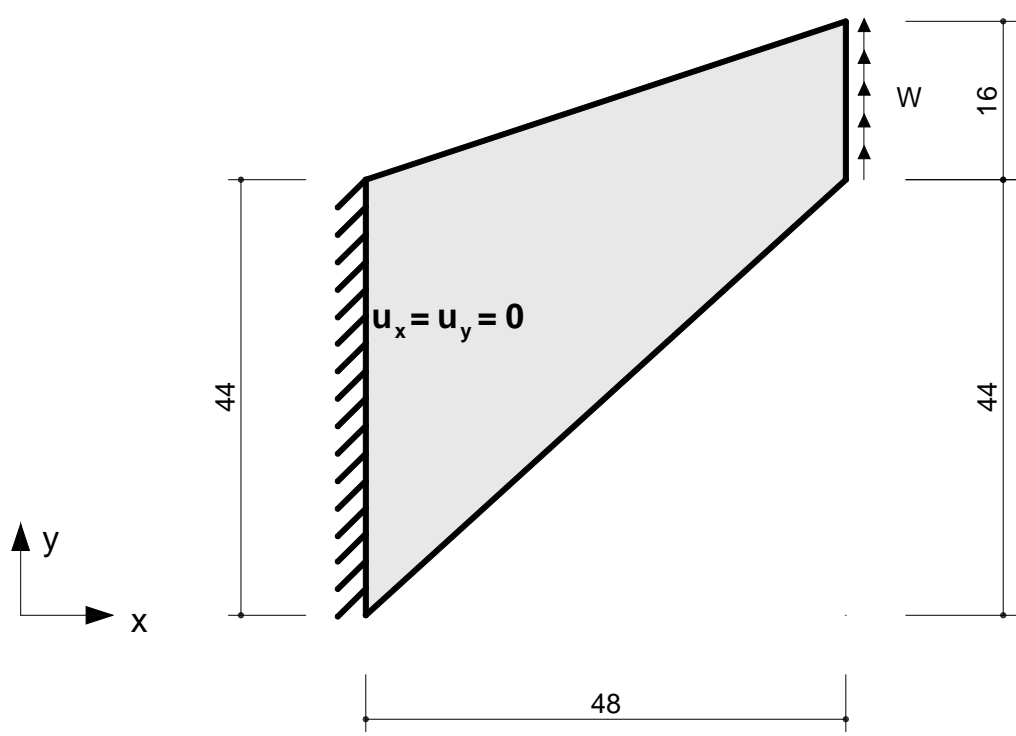

Figura 7.46. Chapa analisada no $5^{\circ}$ problema. Sua geometria, vinculação e carregamento.

A referida chapa tem espessura unitária e dimensões conforme se indicou na figura anterior. As propriedades de interesse do material, módulo de Young e coeficiente de Poisson, apresentam, respectivamente, os seguintes valores: $E=1$ e $v=1 / 3$.

Este problema foi originalmente proposto por Cook (1987), sendo atualmente um 
modelo de referência para testes numéricos.

Para efeito de confronto dos resultados aproximados neste problema realizou-se uma análise via o MEF convencional com o auxílio do software ANSYS $^{\circledR}$ para a obtenção de valores de referência. A discretização envolveu 969 elementos e 2072 nós, conforme ilustrado na Figura (7.47). O elemento utilizado foi igual ao adotado nos três primeiros problemas. Desta forma foi despendido um total de 4144 graus de liberdade.

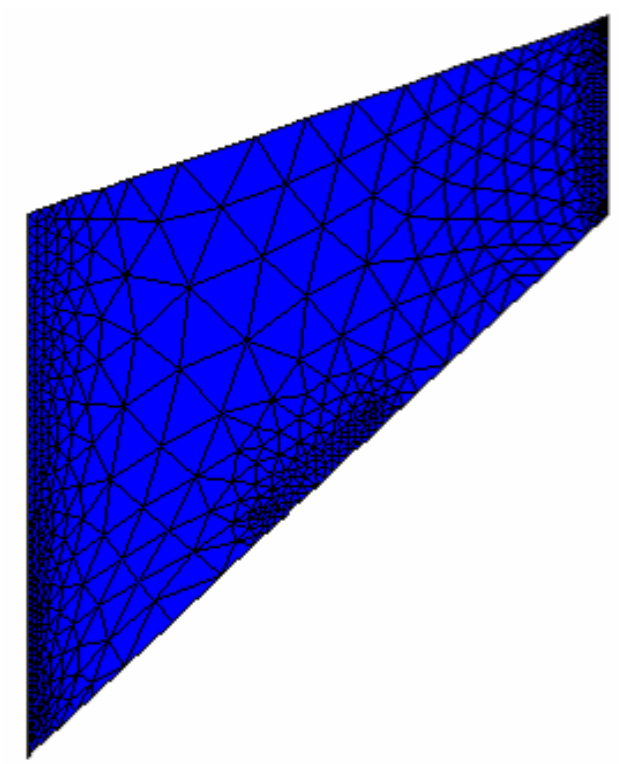

Figura 7.47. Discretização adotada na análise realizada via o MEF clássico para obtenção dos valores de referência do $5^{\circ}$ problema.

Os valores de referência obtidos foram os seguintes: $u_{y_{R E F}}=23,95$ para $o$ deslocamento vertical localizado no centro da aresta direita e $\sigma_{R E F}=0,2368$ para a tensão normal máxima no meio da aresta inferior.

No trabalho de Piltner e Taylor (1999), apresenta-se uma análise comparativa acerca de algumas grandezas aproximadas no problema idealizado por Cook (1987) para algumas formulações similares em elementos finitos encontradas na literatura. Especificamente comparam-se os valores aproximados de deslocamento vertical no ponto A e tensão normal máxima no ponto $\mathrm{B}$, obtidos a partir de discretizações envolvendo quatro, dezesseis $\mathrm{e}$ duzentos e cinqüenta e seis elementos, conforme ilustrado na Figura (7.48). 


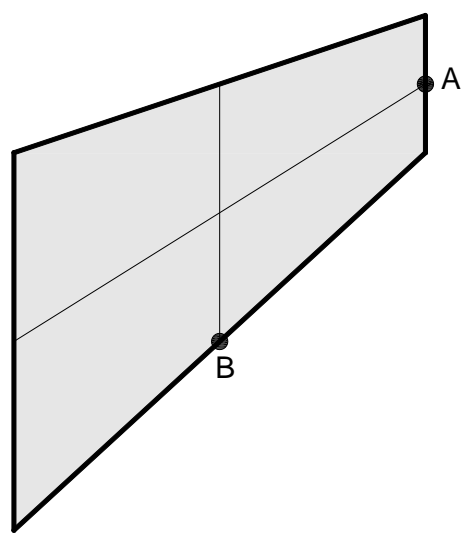

Rede $2 \times 2$

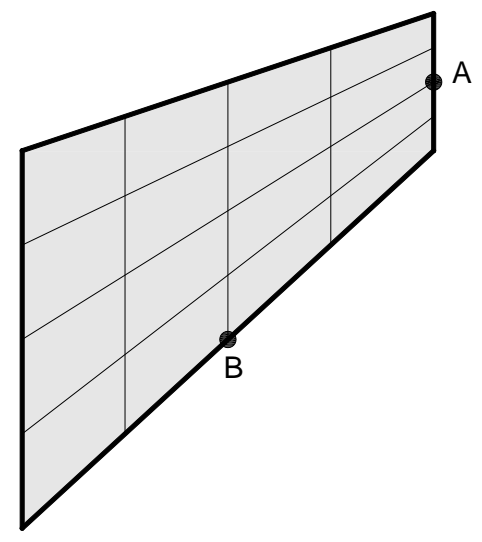

Rede $4 \times 4$

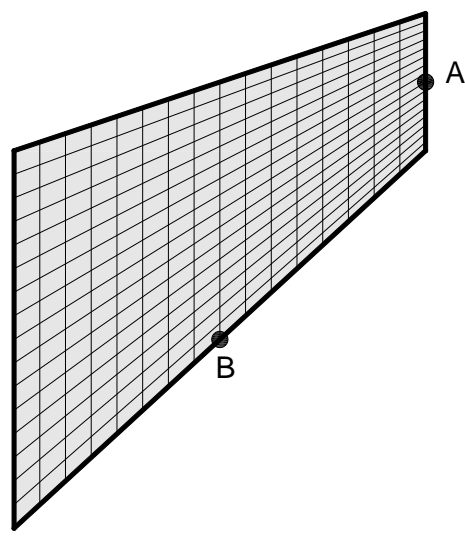

Rede $16 \times 16$

Figura 7.48. Discretizações adotadas na análise comparativa.

Tal análise foi aqui reproduzida para a formulação estudada neste trabalho, ampliando os resultados obtidos no trabalho de Piltner e Taylor (1999).

Assim sendo, considerem-se as seguintes designações dos elementos das formulações que foram averiguadas:

- Q4: Elemento de deslocamento bilinear isoparamétrico (BATHE; WILSON, 1976; HUGHES, 1987; TAIG, 1961).

- QM6: Elemento de deformação (TAYLOR; BERESFORD; WILSON, 1976).

- P-S: Elemento híbrido de tensão (PIAN; SUMIHARA, 1984).

- QE2: Elemento misto (PILTNER; TAYLOR, 1995).

- $\bar{B}-Q E 4:$ Elemento misto (PILTNER; TAYLOR, 1999).

- HTE: Elemento estudado neste trabalho. Utiliza-se ainda a notação da base utilizada no contorno do elemento ( $\left.\beta_{0}, \beta_{1}, \beta_{2} e \beta_{3}\right)$ com a respectiva dimensão (dim).

Salienta-se que em todas as formulações citadas o teste do "mosaico" foi verificado.

Os resultados obtidos nas aproximações do deslocamento vertical do ponto A e da tensão normal máxima no ponto $\mathrm{B}$, para os elementos citados, estão indicados na Tabela (7.11). Particularmente, no elemento da formulação estudada neste trabalho estão apresentados os resultados obtidos para diferentes situações de enriquecimento analisadas, envolvendo as bases de aproximação no contorno: $\beta_{0}, \beta_{1}, \beta_{2}$ e $\beta_{3}$. Na Tabela (7.12) indicam-se os erros relativos obtidos em relação aos valores de referência para as situações consideradas na Tabela (7.11). 
Tabela 7.11 - Resultados aproximados de algumas grandezas de interesse para diferentes formulações em elementos finitos encontradas na literatura

\begin{tabular}{|c|c|c|c|c|c|c|}
\hline \multirow{5}{*}{ Elemento } & \multicolumn{6}{|c|}{ Valores de referência: $u_{y_{R E F}}=23,95$ e $\sigma_{R E F}=0,2368$} \\
\hline & \multicolumn{6}{|c|}{ Discretização } \\
\hline & \multirow{2}{*}{\multicolumn{2}{|c|}{$\begin{array}{c}\text { Rede } 2 \times 2 \\
\begin{array}{c}\text { Valores aproximados nos } \\
\text { pontos de referência }\end{array}\end{array}$}} & \multirow{2}{*}{\multicolumn{2}{|c|}{$\begin{array}{c}\text { Rede } 4 \times 4 \\
\begin{array}{c}\text { Valores aproximados nos } \\
\text { pontos de referência }\end{array}\end{array}$}} & \multirow{2}{*}{\multicolumn{2}{|c|}{$\begin{array}{c}\text { Rede 16x16 } \\
\begin{array}{c}\text { Valores aproximados nos } \\
\text { pontos de referência }\end{array}\end{array}$}} \\
\hline & & & & & & \\
\hline & $\begin{array}{c}\text { Deslocamento } \\
\text { vertical }\end{array}$ & $\begin{array}{c}\text { Tensão } \\
\text { máxima }\end{array}$ & $\begin{array}{c}\text { Deslocamento } \\
\text { vertical }\end{array}$ & $\begin{array}{c}\text { Tensão } \\
\text { máxima }\end{array}$ & $\begin{array}{c}\text { Deslocamento } \\
\text { vertical }\end{array}$ & $\begin{array}{l}\text { Tensão } \\
\text { máxima }\end{array}$ \\
\hline Q4 & 11,85 & 0,1078 & 18,30 & 0,1814 & 23,43 & 0,2353 \\
\hline QM6 & 21,05 & 0,1773 & 23,02 & 0,2225 & 23,88 & 0,2364 \\
\hline $\mathrm{P}-\mathrm{S}$ & 21,13 & 0,1854 & 23,02 & 0,2241 & 23,88 & 0,2364 \\
\hline QE2 & 21,35 & 0,1956 & 23,02 & 0,2261 & 23,88 & 0,2364 \\
\hline$\overline{\mathrm{B}}-\mathrm{QE} 4$ & 21,35 & 0,1956 & 23,02 & 0,2261 & 23,88 & 0,2364 \\
\hline $\operatorname{HTE}\left(\beta_{0}, \operatorname{dim}=2\right)$ & 14,87 & 0,1167 & 20,58 & 0,1917 & 23,68 & 0,2314 \\
\hline $\operatorname{HTE}\left(\beta_{1}, \operatorname{dim}=3\right)$ & 23,73 & 0,2574 & 23,94 & 0,2428 & 23,96 & 0,2373 \\
\hline $\operatorname{HTE}\left(\beta_{1}, \operatorname{dim}=4\right)$ & 24,01 & 0,2361 & 23,96 & 0,2376 & 23,96 & 0,2368 \\
\hline $\operatorname{HTE}\left(\beta_{2}, \operatorname{dim}=3\right)$ & 23,05 & 0,2504 & 23,77 & 0,2393 & 23,95 & 0,2361 \\
\hline $\operatorname{HTE}\left(\beta_{2}, \operatorname{dim}=4\right)$ & 23,98 & 0,2282 & 23,96 & 0,2374 & 23,96 & 0,2368 \\
\hline $\operatorname{HTE}\left(\beta_{3}, \operatorname{dim}=3\right)$ & 23,57 & 0,2545 & 23,88 & 0,2409 & 23,96 & 0,2368 \\
\hline $\operatorname{HTE}\left(\beta_{3}, \operatorname{dim}=4\right)$ & 23,52 & 0,2206 & 23,84 & 0,2419 & 23,96 & 0,2368 \\
\hline
\end{tabular}

Tabela 7.12 - Erros relativos nas aproximações do deslocamento vertical e tensão máxima nos pontos de referência para diferentes formulações em elementos finitos encontradas na literatura.

\begin{tabular}{|c|c|c|c|c|c|c|}
\hline \multirow{5}{*}{ Elemento } & \multicolumn{6}{|c|}{ Valores de referência: $u_{y_{R E F}}=23,95$ e $\sigma_{R E F}=0,2368$} \\
\hline & \multicolumn{6}{|c|}{ Discretização } \\
\hline & \multirow{2}{*}{\multicolumn{2}{|c|}{$\begin{array}{c}\text { Rede } 2 \times 2 \\
\text { Erros relativos (\%) }\end{array}$}} & \multirow{2}{*}{\multicolumn{2}{|c|}{$\begin{array}{l}\text { Kede } 4 \times 4 \\
\text { Erros relativos (\%) }\end{array}$}} & \multirow{2}{*}{\multicolumn{2}{|c|}{$\begin{array}{c}\text { Rede 16x16 } \\
\text { Erros relativos (\%) }\end{array}$}} \\
\hline & & & & & & \\
\hline & $\begin{array}{c}\text { No } \\
\text { deslocamento } \\
\text { vertical } \\
\end{array}$ & $\begin{array}{c}\text { Na } \\
\text { tensão } \\
\text { máxima }\end{array}$ & $\begin{array}{c}\text { No } \\
\text { deslocamento } \\
\text { vertical } \\
\end{array}$ & $\begin{array}{c}\mathrm{Na} \\
\text { tensão } \\
\text { máxima }\end{array}$ & $\begin{array}{c}\text { No } \\
\text { deslocamento } \\
\text { vertical }\end{array}$ & $\begin{array}{c}\text { Na } \\
\text { tensão } \\
\text { máxima }\end{array}$ \\
\hline $\mathrm{Q} 4$ & 50,52 & 54,48 & 23,59 & 23,40 & 2,17 & 0,63 \\
\hline QM6 & 12,11 & 25,13 & 3,88 & 6,04 & 0,29 & 0,17 \\
\hline P-S & 11,77 & 21,71 & 3,88 & 5,36 & 0,29 & 0,17 \\
\hline QE2 & 10,86 & 17,40 & 3,88 & 4,52 & 0,29 & 0,17 \\
\hline$\overline{\mathrm{B}}-\mathrm{QE} 4$ & 10,86 & 17,40 & 3,88 & 4,52 & 0,29 & 0,17 \\
\hline $\operatorname{HTE}\left(\beta_{0}, \operatorname{dim}=2\right)$ & 37,91 & 50,72 & 14,07 & 19,05 & 1,13 & 2,28 \\
\hline $\operatorname{HTE}\left(\beta_{1}, \operatorname{dim}=3\right)$ & 0,92 & $-8,70$ & 0,04 & $-2,53$ & $-0,04$ & $-0,21$ \\
\hline $\operatorname{HTE}\left(\beta_{1}, \operatorname{dim}=4\right)$ & $-0,25$ & 0,30 & $-0,04$ & $-0,34$ & $-0,04$ & 0,00 \\
\hline $\operatorname{HTE}\left(\beta_{2}, \operatorname{dim}=3\right)$ & 3,76 & $-5,74$ & 0,75 & $-1,06$ & 0,00 & 0,30 \\
\hline $\operatorname{HTE}\left(\beta_{2}, \operatorname{dim}=4\right)$ & $-0,13$ & 3,63 & $-0,04$ & $-0,25$ & $-0,04$ & 0,00 \\
\hline $\operatorname{HTE}\left(\beta_{3}, \operatorname{dim}=3\right)$ & 1,59 & $-7,47$ & 0,29 & $-1,73$ & $-0,04$ & 0,00 \\
\hline $\operatorname{HTE}\left(\beta_{3}, \operatorname{dim}=4\right)$ & 1,80 & 6,84 & 0,46 & $-2,15$ & $-0,04$ & 0,00 \\
\hline
\end{tabular}

Percebe-se das Tabelas (7.11) e (7.12) que os resultados obtidos pela formulação híbrida-Trefftz de tensão apontaram, na maioria das vezes, para uma boa capacidade de 
aproximação. À exceção da situação não enriquecida (HTE com a base $\beta_{0}$ ) os valores aproximados convergiram significativamente para os valores de referência, com o aumento do refino da rede. Além disso, verificou-se que a utilização de bases com dimensões não inferiores a 3 já foram suficientes para gerar boas respostas, mesmo para a discretização com apenas quatro elementos.

Comparado aos outros elementos averiguados, o elemento HTE com $\operatorname{dim} \geq 3$ apresentou, geralmente, erros relativos menores, para todas as bases de aproximação no contorno exploradas.

Ressalta-se que as observações acerca dos resultados das Tabelas (7.11) e (7.12) são meramente qualitativas, uma vez que não foram computados os graus de liberdade envolvidos nas análises.

Objetivando-se averiguar as taxas de convergência das grandezas analisadas determinaram-se as curvas que quantificam os erros relativos das aproximações em função dos graus de liberdade envolvidos nas discretizações indicadas na Figura (7.48). Nas Figuras (7.49) e (7.50) encontram-se, respectivamente, as curvas que quantificam os erros do deslocamento vertical e da tensão normal máxima de referência para os diferentes elementos que foram comparados.

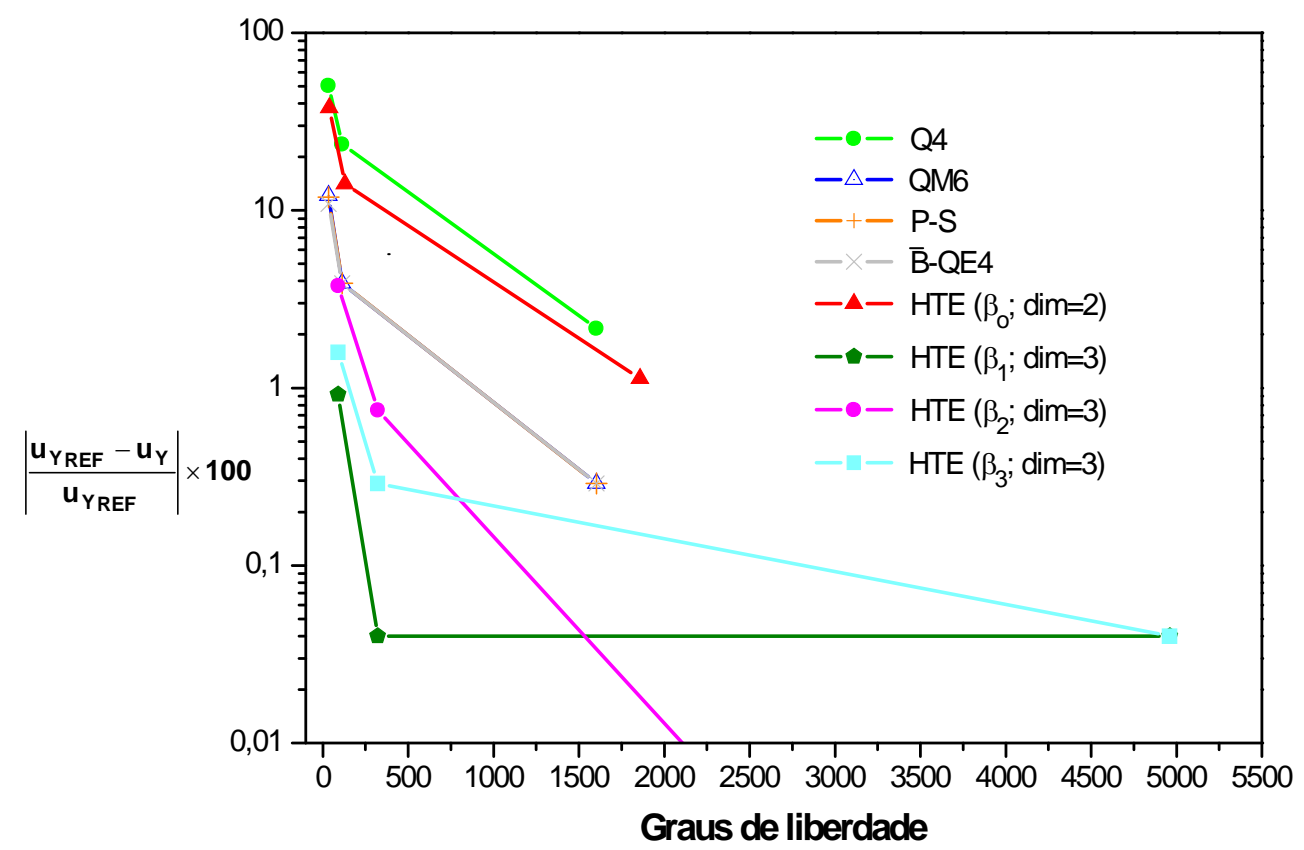

Figura 7.49. Refino-h. Erro relativo do deslocamento vertical no ponto de referência (\%). 


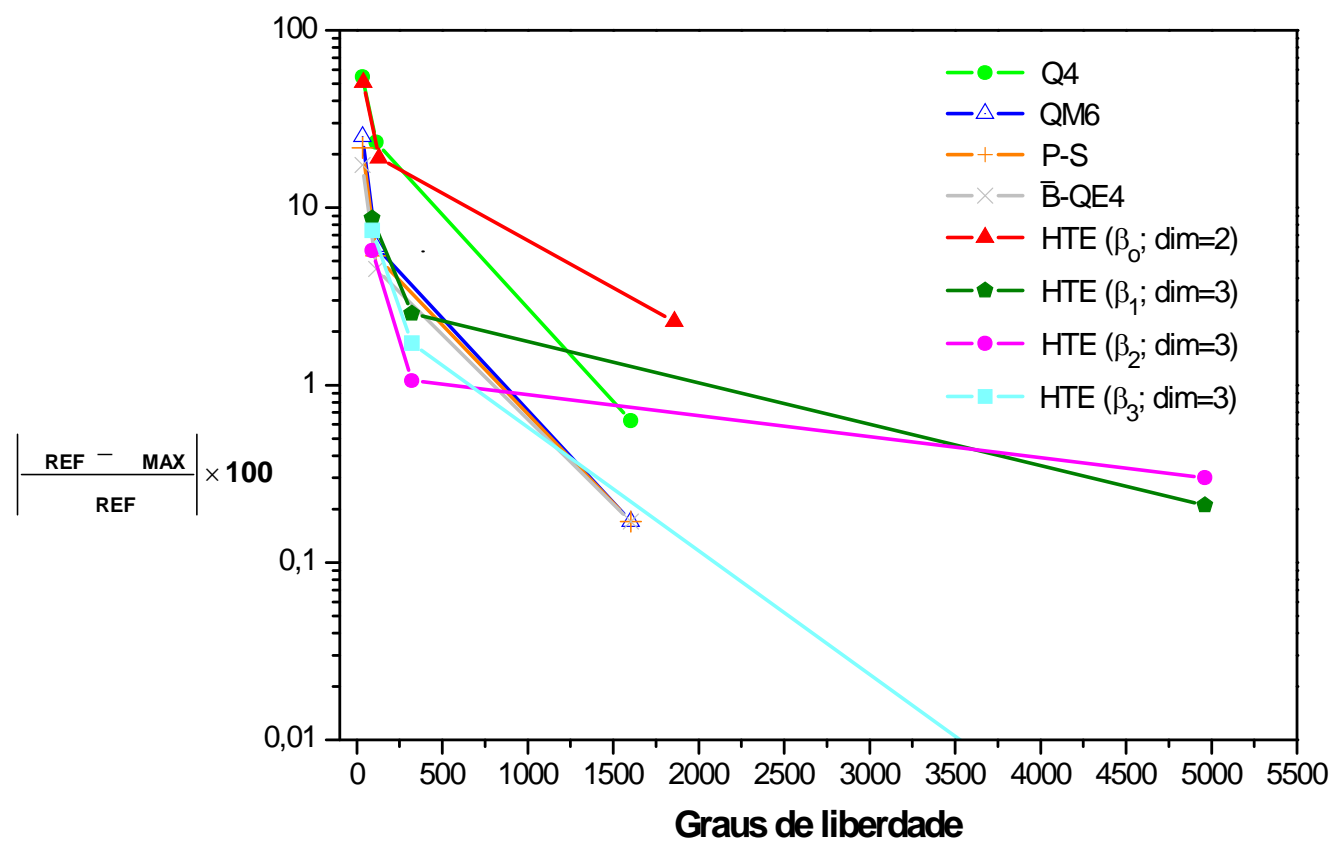

Figura 7.50. Refino-h. Erro relativo da tensão normal máxima no ponto de referência (\%).

Percebe-se na Figura (7.49) que, à exceção do elemento HTE com dim = 2, nas outras situações de enriquecimento consideradas o desempenho da formulação estudada foi excelente, caracterizado por elevadas taxas de convergência. Comparado aos outros elementos os erros relativos obtidos foram menores, mesmo para um número reduzido de graus de liberdade.

Por outro lado, na aproximação da tensão normal máxima os resultados obtidos foram menos notáveis, mais ainda muito bons, conforme ilustrado na Figura (7.50). Nas situações onde se utilizou o elemento HTE com $\operatorname{dim}=3$, na maioria das vezes, constataram-se erros relativos menores, principalmente na discretização mais simples considerada. Além disso, pode-se perceber que as taxas de convergências iniciais apresentaram a mesma magnitude daquelas obtidas pelos outros elementos que foram comparados. 


\section{Considerações finais e conclusões}

Inserido no âmbito das formulações não convencionais em elementos finitos este trabalho pretendeu fornecer uma contribuição ao estudo da formulação híbrida-Trefftz de tensão.

A concepção primordial desta pesquisa, relativa ao enriquecimento de zonas de interesse no domínio estrutural, mostrou-se promissora. Designado neste trabalho de "enriquecimento seletivo" a combinação da forma clássica de refino-p do MEF com a estrutura de enriquecimento centrada em nuvens proposta pelo MEFG, assente sobre a formulação híbrida-Trefftz de tensão, apresentou-se como uma alternativa interessante aos procedimentos h-adaptativos ou p-adaptativos, sobretudo em termos de custo computacional.

Conceitualmente, a adequação da formulação híbrida-Trefftz ao enriquecimento do campo de deslocamentos, merece destaque. Em virtude do sistema unidimensional de coordenadas locais, definido nas fronteiras estáticas dos elementos, uma nova base enriquecida de aproximação pode ser obtida sem maiores dificuldades. Em outras formulações, a aplicação da técnica sistemática de enriquecimento preconizada pelo MEFG, gera, na maioria das vezes, uma família de funções linearmente dependente, exigindo algoritmos específicos de eliminação de dependências lineares do sistema resolutivo. Neste contexto cita-se, por exemplo, o trabalho de Góis (2004), onde o procedimento de eliminação de dependências lineares proposto por Strouboulis, Babuška e Copps (2000) foi adaptado para viabilizar a incorporação das técnicas de enriquecimento nodal do MEFG na formulação híbrido-mista de tensão.

Como parte dos objetivos deste trabalho, foi fornecida uma contribuição, ainda que de pequena monta, ao estudo de sólidos com múltiplas fissuras mediante o desenvolvimento do Método da Partição em formulação híbrida-Trefftz com enriquecimento seletivo, apresentada no capítulo 5. Foram estabelecidos os procedimentos necessários para uma implementação mais geral do método para um número ilimitado de fissuras, considerando-se fissuras inclinadas e carregamentos fictícios nas direções paralela e perpendicular às faces da fissura.

Acerca das análises numéricas relatadas no capítulo 7 obtiveram-se as seguintes conclusões de significativa relevância: 
- No $1^{\circ}$ problema ficou patente que o enriquecimento exclusivo no domínio dos elementos não promoveu melhorias em nenhum dos campos aproximados, acarretando apenas num aumento do número de graus de liberdade envolvido.

- No $2^{\circ}$ problema as simulações numéricas nos testes de distorção de rede indicaram que apenas o elemento não enriquecido apresentou sensibilidade significativa à distorção. Nas outras situações de enriquecimento analisadas os elementos mostraram-se extremamente robustos.

- No $3^{\circ}$ problema constatou-se que a utilização de elementos com orifício circular é uma alternativa numericamente eficaz, sobretudo na aproximação do campo de tensões, além de evitar aproximações de geometria. Contudo, tal procedimento deve ser realizado com certas ressalvas. Notou-se que nos elementos com orifício, onde a dimensão do raio era consideravelmente inferior às dimensões dos lados do elemento, a norma do erro do sistema resolvente era significativa. Desta forma motivam-se novos estudos que viabilizem a utilização de elementos que superem tais dificuldades.

- O $4^{\circ}$ problema serviu para averiguar a eficiência do Método da Partição em formulação híbrida-Trefftz com enriquecimento seletivo. Para uma única fissura no domínio o método mostrou-se eficiente, uma vez que foi verificada tanto a convergência no fator de concentração de tensões quanto uma distribuição de tensões conforme a esperada, com zonas de concentração de tensões na região de singularidade e tensões nulas nas faces da fissura. Contudo, apenas com os resultados obtidos, não é possível julgar as reais potencialidades do referido método, uma vez que a simplificação adotada na implementação ( $\Gamma_{f} \equiv$ contorno de $V_{f}$ ) pode ter influenciado negativamente na resposta, pelo menos na saída gráfica de tensões na região do contorno de $\mathrm{V}_{\mathrm{f}}$ isto ficou patente. Além disso, na extração do fator de intensidade de tensões utilizou-se a técnica de correlação dos deslocamentos, a qual pode não ter sido, necessariamente, a mais adequada.

- No $5^{\circ}$ problema realizou-se uma análise comparativa entre alguns elementos encontrados na literatura. Os resultados apontaram que a formulação híbrida-Trefftz de tensão apresenta uma notável capacidade de aproximação quando se utilizam bases de aproximação com dimensão não inferior a 3 nas fronteiras estáticas dos elementos. Na maioria das vezes, um número reduzido de graus de liberdade foi suficiente para gerar respostas melhores do que os resultados das outras formulações averiguadas. 
- Em todas as análises onde se utilizaram as três bases de aproximação no contorno $\left(\beta_{1}\right.$, $\beta_{2} \mathrm{e} \beta_{3}$ ) ficou evidente que todas elas se adequaram bem à formulação estudada, não existindo diferenças apreciáveis nas respostas obtidas. Ressalta-se que a utilização de tais bases evitou procedimentos específicos de eliminação de dependências lineares no sistema resolutivo.

- Nos problemas onde se utilizou o enriquecimento seletivo, os resultados obtidos indicaram que a formulação proposta é uma alternativa numérica eficaz, além de evitar refinamentos desnecessários. De fato o enriquecimento de uma região restrita no domínio estrutural formada por um número reduzido de fronteiras estáticas e elementos foi suficiente para garantir bons resultados, sobretudo na aproximação do campo de tensões.

Finalmente, pode-se concluir que a formulação híbrida-Trefftz de tensão apresenta uma elevada capacidade de aproximação e robustez numérica. Aliada à idéia do enriquecimento seletivo a referida formulação passa a ter maior potencialidade, permitindo racionalizar o dispêndio computacional inerente à maioria das análises numéricas.

Apesar da avaliação positiva deste trabalho algumas sugestões para o seu aperfeiçoamento ou para desenvolvimentos futuros podem ser fornecidas, quais sejam:

- Incorporação de outros elementos com características especiais à estrutura desenvolvida do programa, tais como: elementos com cavidade elíptica, elementos com fissuras, elementos com cargas concentradas aplicadas no domínio ou contorno, entre outros.

- Realização de um estudo mais aprofundado do Método da Partição em formulação híbrida-Trefftz com enriquecimento seletivo. Inicialmente novas técnicas de extração dos fatores de intensidade de tensões deveriam ser analisadas, visando-se a uma maior adequação. Em seguida, uma implementação numérica mais ampla e sem simplificações $\left(\Gamma_{f} \neq\right.$ contorno de $V_{f}$ e carregamento fictício nas direções paralela e perpendicular às faces da fissura) poderia ser efetuada. Neste contexto, seria oportuno realizar simulações numéricas em domínios contendo múltiplas fissuras no âmbito da confiabilidade estrutural, de forma a definir estados limites últimos.

- Por fim, trabalhos similares poderiam ser realizados com outras formulações não convencionais, tais como, a formulação híbrida-Trefftz de deslocamento ou mesmo formulações híbridas puras. 


\section{Bibliografia}

BABUŠKA, I; ANDERSSON, B. (2005). The splitting method as a tool for multiple damage analysis. Siam Journal of Scientific Computing, v.26, n.4, p.1114-1145.

BATHE, K. J. (1996). Finite element procedures. 2. ed. Prentice-Hall.

BATHE, K. J.; WILSON, E. L. (1976). Numerical Methods in Finite element Analysis. Prentice-Hall, Englewood Cliffs, N. J..

BITENCOURT, T.N et al. (2003). Fatigue life and crack path predictions in generic 2D structural components. Engineering Fracture Mechanics, v.72, p.2174-2185.

BUSSAMRA, F.L. S. (1999). Elementos finitos híbrido-trefftz: Um modelo elasto-plástico tridimensional. Tese (Doutorado). Escola Politécnica da Universidade de São Paulo.

BUSSAMRA, F. L. S.; PIMENTA, P. M.; FREITAS, J. A. T. (2001). Hybrid-Trefftz stress elements for three-dimensional elastoplasticity. Computer Assisted Mechanics and Engineering Sciences, v.8, p.235-246.

CHANG, J. R. et al. (2002). Applications of the direct Trefftz boundary element method to the free-vibration problem of a membrane. Journal acoustic society American, v.112, p.518527.

COOK, R. (1987). A plane hybrid element with rotational d.o.f and adjustable stiffiness. International Journal for Numerical Methods in Engineering, v.24, p.1499-1508.

DÍAZ, M.; HERREIRA, I.; YATES, R. (2002). Indirect method of collocation. $14^{\circ}$ international conference on domain decomposition methods, Cocoyoc, Mor., Mexico, (www.ddm.org).

DUARTE, C. A.; BABUŠKA, I; ODEN, J. T. (2000). Generalized finite element methods for three-dimensional structural mechanics problems. Computer \& Structures, v.77, n.2, p.215232.

FERNANDEZ, C. M. T. T. (1998). Utilização e desenvolvimento de uma formulação indirecta de Trefftz na análise de lajes finas. Dissertação (Mestrado) - Universidade Técnica de Lisboa, Portugal, Lisboa.

FREITAS, J. A. T. (1997). Hybrid-Trefftz displacement and stress elements for elastodynamic analysis in the frequency domain. Computer Assisted Mechanics and Engineering Sciences, v.4, p.345-368. 
FREITAS, J. A. T. (1998). Formulation of elastostatic hybrid-Trefftz stress elements. Computer Methods in Applied Mechanics and Engineering. v.153, p.127-151.

FREITAS, J. A. T.; ALMEIDA, J. P. B. M.; PEREIRA, E. M. B. R. (1999). NonConventional formulations for the finite element method. Computational Mechanics, v.23, p. 488-501.

FUNG, Y. C. (1965). Foundations of Solid Mechanics, Prentice-Hall,Englewood Cliffs, NJ.

GÓIS, W. (2004). Método dos elementos finitos generalizados em formulação variacional mista. Dissertação (Mestrado) - Escola de Engenharia de São Carlos, Universidade de São Paulo, São Carlos.

HOFFMAN, K.; KUNZE, R. (1971). Álgebra linear. Editora Polígono S. A.

HUGHES, T. J. R. (1987). The Finite Element Method. Prentice-Hall, Englewood Cliffs, N. J..

JIN, F. S.; QIN, Q. H. (1995). Variational principle and hybrid Trefftz finite element for the analysis of Reissner plates. Computer \& Structures, v.56, n.4, p.697-701.

JIROUSEK, J. (1978). Basis for development of large finite elements locally satisfying all fields equations. Computer Methods in Applied Mechanics and Engineering, v.14, p. 65-92.

JIROUSEK, J.; QIN, Q. H. (1996). Application of hybrid-Trefftz element approach to transient heat conduction analysis. Computer \& Structures, v.58, p.195-201.

JIROUSECK, J.; VENKATESH, A. (1992). Hybrid Trefftz plane elasticity elements with pmethod capabilities. International Journal for Numerical Methods in Engineering, v. 35, p.1443-1472.

JIROUSEK, J.; WRÓBLEWSKI, A. (1996).T-elements: state of the art and future trends. Archives of Computational Methods in Engineering, v.3, n.4, p.323-434.

LEITÃO, V. M. A. (2001). Comparison of Galerkin and collocation Trefftz formulations for plane elasticity. Computer Assisted Mechanics and Engineering Sciences, v.8, p.397-407.

LEONEL, E. D. (2006). Método dos elementos de contorno aplicado à análise de corpos multi-fraturados. Dissertação (Mestrado) - Escola de Engenharia de São Carlos, Universidade de São Paulo, São Carlos.

MUSKHELISHVILI, N. I. (1953). Some basic problems of the mathematical theory of elasticity. Noordhoof, Groningen, Holland.

PEREIRA, J. P. A. (2004). Extração de fatores de intensidade de tensão utilizando a solução do método dos elementos finitos generalizados. Dissertação (Mestrado) - Escola de 
engenharia de São Carlos, Universidade de São Paulo, São Carlos.

PETERSON, R. E. (1974). Stress Concentration Factors, Wiley \& Sons

PETROLITO, J. (1996). Triangular thick plate elements based on a hybrid-Trefftz approach. Computer \& Structures, v.60, p.883-894.

PIAN, T. H. H.; SUMIHARA, K.(1984). Racional approach for assumed stress elements. International Journal for Numerical Methods in Engineering, v.20, p.1685-1695.

PILTNER, R.; TAYLOR, R. L.(1995). A quadrilateral finite element with two enhanced strain modes. International Journal for Numerical Methods in Engineering, v.38, p.17831808.

PILTNER, R.; TAYLOR, R. L. (1999). Systematic construction of B-bar functions for linear and non-linear mixed-enhanced finite element for plane elasticity problems. International Journal for Numerical Methods in Engineering, v.44, n.5, p.615-639.

PIMENTA, P. M.; PROENÇA, S. P. B.; FREITAS, J. A. T. (2002). Elementos finitos híbridos-mistos com enriquecimento nodal.J.M.Gaicolea, C. Mota Soares, M. Pastor y G. Bugeda (Eds.), Métodos Numéricos in Ingeniería V, SEMNI.

QIN, Q. H. (1996). Nonlinear analysis of thick plates by HT FE approach. Computer \& Structures, v.61, p.271-281.

QIN, Q. H. (2003). Variational formulations for TFEM of piezoelectricity. International Journal of Solids Structures, v.40, p.6335-6346.

QIN, Q. H. (2005). Trefftz Finite Element Method and its applications. Applied Mechanics Reviews, v.58, p. 316-336.

STROUBOLIS, T.; BABUŠKA, I.; COPPS, K. (2000). The design and analysis of the generalized finite element method. Computer Methods in Applied Mechanics and Engineering, v.181, n. 1-3, p. 43-69.

SWOKOWSKI, E. W. (1983). Cálculo com geometria analítica. McGraw-Hill do Brasil.

TAIG, I. C. (1961). Structural analysis by the matrix displacement method. English Electric Aviation Report, n. S017.

TAYLOR, R. L.; BERESFORD, P. J.; WILSON, E. L.(1976). A non-conforming element for stress analysis. International Journal for Numerical Methods in Engineering, v.10, p.12111219.

TIMOSHENKO, S. P.; GOODIER, J.N. (1980). Teoria da Elasticidade.Guanabara Dois S.A. 
VALLIAPPAN, S. (1981).Continuum Mechanics: Fundamentals. A. A. Balkema.

VÖRÖS, G. M.; JIROUSECK, J. (1991). Application of the hybrid-Trefftz finite element model to thin shell analysis. P. Ladeveze and O. C. Zienckewicz (Eds.) Procedures European conference on new advances in computer structures mechanics, Elsevier, Giens, France, p. 547-554.

WRÓBLEWSKI, A.; ZIELINSKI, A. P.; JIROUSECK, J. (1992). Hybrid-Trefftz p-element for 3-D axisymmetric problems of elasticity. Numerical methods in engineering. C. Hirsch, $\mathrm{O}$. C. Zienckiewicz, and E. Onãte (Eds.). Procedures First European conference on numerical methods in engineering., Elsevier, Brussels, p.803-810.

ZIENKIEWICZ, O. C. et al. (1986). The patch test for mixed formulation. International journal for numerical methods in engineering, v.23, p.1873-1882.

ZIENKIEWICZ, O. C.; TAYLOR, R. L. (2000). The finite element method-Volume 1: The Basis.5.ed. Butterworth Heinemann. 
Apêndice A - Matrizes utilizadas na aproximação dos campos de deslocamentos e tensões em domínios contínuos: construção a partir da equação de Navier

As matrizes apresentadas a seguir foram obtidas com a utilização do s oftware Mathematica ${ }^{\circledR}$.

- Matriz $\mathrm{N}_{\mathrm{c}}$ :

Da $1^{\mathrm{a}}$ à $9^{\mathrm{a}}$ coluna:

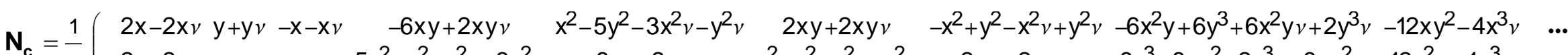

Da $10^{\mathrm{a}}$ à $13^{\mathrm{a}}$ coluna :

$3 \mathrm{x}^{2} \mathrm{y}-\mathrm{y}^{3}+3 \mathrm{x}^{2} \mathrm{y} v-\mathrm{y}^{3} v-\mathrm{x}^{3}+3 \mathrm{xy}^{2}-\mathrm{x}^{3} v+3 \mathrm{xy}^{2} v \quad-4 \mathrm{x}^{3} \mathrm{y}+20 \mathrm{x} \mathrm{y}^{3}+12 \mathrm{x}^{3} \mathrm{y} v+4 \mathrm{xy}^{3} v \quad-\mathrm{x}^{4}-18 \mathrm{x}^{2} \mathrm{y}^{2}+7 \mathrm{y}^{4}-5 \mathrm{x}^{4} v+6 \mathrm{x}^{2} \mathrm{y}^{2} v+3 \mathrm{y}^{4} v \quad \ldots$

$x^{3}-3 x y^{2}+x^{3} v-3 x y^{2} v \quad 3 x^{2} y-y^{3}+3 x^{2} y v-y^{3} v \quad 7 x^{4}-18 x^{2} y^{2}-y^{4}+3 x^{4} v+6 x^{2} y^{2} v-5 y^{4} v \quad 20 x^{3} y-4 x y^{3}+4 x^{3} y v+12 x y^{3} v$

Da $14^{\mathrm{a}}$ à $17^{\mathrm{a}}$ coluna:

$4 x^{3} y-4 x y^{3}+4 x^{3} y v-4 x y^{3} v \quad-x^{4}+6 x^{2} y^{2}-y^{4}-x^{4} v+6 x^{2} y^{2} v-y^{4} v \quad 40 x^{2} y^{3}-8 y^{5}+20 x^{4} y v-4 y^{5} v-2 x^{5}-20 x^{3} y^{2}+30 x y^{4}-6 x^{5} v+20 x^{3} y^{2} v+10 x y^{4} v \quad . .$.

$\mathrm{x}^{4}-6 \mathrm{x}^{2} \mathrm{y}^{2}+\mathrm{y}^{4}+\mathrm{x}^{4} v-6 \mathrm{x}^{2} \mathrm{y}^{2} v+\mathrm{y}^{4} v \quad 4 \mathrm{x}^{3} \mathrm{y}-4 \mathrm{x}^{3}+4 \mathrm{x}^{3} \mathrm{y} v-4 \mathrm{x} \mathrm{y}^{3} v \quad 8 \mathrm{x}^{5}-40 \mathrm{x}^{3} \mathrm{y}^{2}+4 \mathrm{x}^{5} v-20 \mathrm{x} \mathrm{y}^{4} v \quad 30 \mathrm{x}^{4} \mathrm{y}-20 \mathrm{x}^{2} \mathrm{y}^{3}-2 \mathrm{y}^{5}+10 \mathrm{x}^{4} \mathrm{y} v+20 \mathrm{x}^{2} \mathrm{y}^{3} v-6 \mathrm{y}^{5} v$ 
Apêndice A: Matrizes utilizadas na aproximação dos campos de deslocamentos e tensões em domínios contínuos

131

Da $18^{\mathrm{a}}$ à $20^{\mathrm{a}}$ coluna:

$$
\begin{array}{lll}
5 x^{4} y-10 x^{2} y^{3}+y^{5}+5 x^{4} y v-10 x^{2} y^{3} v+y^{5} v-x^{5}+10 x^{3} y^{2}-5 x y^{4}-x^{5} v+10 x^{3} y^{2} v-5 x y^{4} v & 6 x^{5} y+60 x^{3} y^{3}-42 x y^{5}+30 x^{5} y v-20 x^{3} y^{3} v-18 x y^{5} v \\
x^{5}-10 x^{3} y^{2}+5 x y^{4}+x^{5} v-10 x^{3} y^{2} v+5 x y^{4} v & 5 x^{4} y-10 x^{2} y^{3}+y^{5}+5 x^{4} y v-10 x^{2} y^{3} v+y^{5} v & 9 x^{6}-75 x^{4} y^{2}+15 x^{2} y^{4}+3 y^{6}+5 x^{6} v-15 x^{4} y^{2} v-45 x^{2} y^{4} v+7 y^{6} v
\end{array}
$$

$21^{\mathrm{a}}$ e $22^{\mathrm{a}}$ colunas:

$$
\begin{array}{ccc}
-3 x^{6}-15 x^{4} y^{2}+75 x^{2} y^{4}-9 y^{6}-7 x^{6} v+45 x^{4} y^{2} v+15 x^{2} y^{4} v-5 y^{6} v & 6 x^{5} y-20 x^{3} y^{3}+6 x y^{5}+6 x^{5} y v-20 x^{3} y^{3} v+6 x y^{5} v & \cdots \\
42 x^{5} y-60 x^{3} y^{3}-6 x y^{5}+18 x^{5} y v+20 x^{3} y^{3} v-30 x y^{5} v & x^{6}-15 x^{4} y^{2}+15 x^{2} y^{4}-y^{6}+x^{6} v-15 x^{4} y^{2} v+15 x^{2} y^{4} v-y^{6} v
\end{array} \quad \cdots
$$

$23^{\mathrm{a}}$ e $24^{\mathrm{a}}$ colunas:

$$
\begin{array}{ccc}
-x^{6}+15 \mathrm{x}^{4} \mathrm{y}^{2}-15 \mathrm{x}^{2} \mathrm{y}^{4}+\mathrm{y}^{6}-\mathrm{x}^{6} v+15 \mathrm{x}^{4} \mathrm{y}^{2} v-15 \mathrm{x}^{2} \mathrm{y}^{4} v+\mathrm{y}^{6} v & 14 \mathrm{x}^{6} \mathrm{y}+70 \mathrm{x}^{4} \mathrm{y}^{3}-126 \mathrm{x}^{2} \mathrm{y}^{5}+10 \mathrm{y}^{7}+42 \mathrm{x}^{6} \mathrm{y} v-70 \mathrm{x}^{4} \mathrm{y}^{3} v-42 \mathrm{x}^{2} \mathrm{y}^{5} v+6 \mathrm{y}^{7} v & \ldots \\
6 \mathrm{x}^{5} \mathrm{y}-20 \mathrm{x}^{3} \mathrm{y}^{3}+6 \mathrm{x} \mathrm{y}^{5}+6 \mathrm{x}^{5} \mathrm{y} v-20 \mathrm{x}^{3} \mathrm{y}^{3} v+6 \mathrm{x} \mathrm{y}^{5} v & 10 \mathrm{x}^{7}-126 \mathrm{x}^{5} \mathrm{y}^{2}+70 \mathrm{x}^{3} \mathrm{y}^{4}+14 \mathrm{xy}^{6}+6 \mathrm{x}^{7} v-42 \mathrm{x}^{5} \mathrm{y}^{2} v-70 \mathrm{x}^{3} \mathrm{y}^{4} v+42 \mathrm{x} \mathrm{y}^{6} v &
\end{array}
$$

$25^{\mathrm{a}}$ e $26^{\mathrm{a}}$ colunas:

$$
\begin{array}{lll}
-4 x^{7}+140 x^{3} y^{4}-56 x y^{6}-8 x^{7} v+84 x^{5} y^{2} v-28 x y^{6} v & 7 x^{6} y-35 x^{4} y^{3}+21 x^{2} y^{5}-y^{7}+7 x^{6} y v-35 x^{4} y^{3} v+21 x^{2} y^{5} v-y^{7} v & \ldots \\
56 x^{6} y-140 x^{4} y^{3}+4 y^{7}+28 x^{6} y v-84 x^{2} y^{5} v+8 y^{7} v & x^{7}-21 x^{5} y^{2}+35 x^{3} y^{4}-7 x y^{6}+x^{7} v-21 x^{5} y^{2} v+35 x^{3} y^{4} v-7 x y^{6} v
\end{array}
$$

$27^{\mathrm{a}}$ e $28^{\mathrm{a}}$ colunas:

$$
\begin{aligned}
& -x^{7}+21 x^{5} y^{2}-35 x^{3} y^{4}+7 x y^{6}-x^{7} v+21 x^{5} y^{2} v-35 x^{3} y^{4} v+7 x y^{6} v \quad 24 x^{7} y+56 x^{5} y^{3}-280 x^{3} y^{5}+72 x y^{7}+56 x^{7} y v-168 x^{5} y^{3} v-56 x^{3} y^{5} v+40 x y^{7} v \\
& 7 x^{6} y-35 x^{4} y^{3}+21 x^{2} y^{5}-y^{7}+7 x^{6} y v-35 x^{4} y^{3} v+21 x^{2} y^{5} v-y^{7} v 11 x^{8}-196 x^{6} y^{2}+210 x^{4} y^{4}+28 x^{2} y^{6}-5 y^{8}+7 x^{8} v-84 x^{6} y^{2} v-70 x^{4} y^{4} v+140 x^{2} y^{6} v-9 y^{8} v \\
& 29^{\mathrm{a}} \text { e } 30^{\mathrm{a}} \text { colunas: } \\
& -5 x^{8}+28 x^{6} y^{2}+210 x^{4} y^{4}-196 x^{2} y^{6}+11 y^{8}-9 x^{8} v+140 x^{6} y^{2} v-70 x^{4} y^{4} v-84 x^{2} y^{6} v+7 y^{8} v \\
& 72 x^{7} y-280 x^{5} y^{3}+56 x^{3} y^{5}+24 x y^{7}+40 x^{7} y v-56 x^{5} y^{3} v-168 x^{3} y^{5} v+56 x y^{7} v \\
& 8 x^{7} y-56 x^{5} y^{3}+56 x^{3} y^{5}-8 x y^{7}+8 x^{7} y v-56 x^{5} y^{3} v+56 x^{3} y^{5} v-8 x y^{7} v \\
& x^{8}-28 x^{6} y^{2}+70 x^{4} y^{4}-28 x^{2} y^{6}+y^{8}+x^{8} v-28 x^{6} y^{2} v+70 x^{4} y^{4} v-28 x^{2} y^{6} v+y^{8} v
\end{aligned}
$$


$31^{\mathrm{a}}$ e $32^{\mathrm{a}}$ colunas:

$-x^{8}+28 x^{6} y^{2}-70 x^{4} y^{4}+28 x^{2} y^{6}-y^{8}-x^{8} v+28 x^{6} y^{2} v-70 x^{4} y^{4} v+28 x^{2} y^{6} v-y^{8} v 36 x^{8} y-504 x^{4} y^{5}+288 x^{2} y^{7}-12 y^{9}+72 x^{8} y v-336 x^{6} y^{3} v+144 x^{2} y^{7} v-8 y^{9} v$ $8 x^{7} y-56 x^{5} y^{3}+56 x^{3} y^{5}-8 x y^{7}+8 x^{7} y v-56 x^{5} y^{3} v+56 x^{3} y^{5} v-8 x y^{7} v \quad 12 x^{9}-288 x^{7} y^{2}+504 x^{5} y^{4}-36 x y^{8}+8 x^{9} v-144 x^{7} y^{2} v+336 x^{3} y^{6} v-72 x y^{8} v$

$33^{\text {a }}$ coluna:

$-6 x^{9}+72 x^{7} y^{2}+252 x^{5} y^{4}-504 x^{3} y^{6}+90 x y^{8}-10 x^{9} v+216 x^{7} y^{2} v-252 x^{5} y^{4} v-168 x^{3} y^{6} v+54 x y^{8} v$

$90 x^{8} y-504 x^{6} y^{3}+252 x^{4} y^{5}+72 x^{2} y^{7}-6 y^{9}+54 x^{8} y v-168 x^{6} y^{3} v-252 x^{4} y^{5} v+216 x^{2} y^{7} v-10 y^{9} v$

$34^{\mathrm{a}}$ coluna:

$9 x^{8} y-84 x^{6} y^{3}+126 x^{4} y^{5}-36 x^{2} y^{7}+y^{9}+9 x^{8} y v-84 x^{6} y^{3} v+126 x^{4} y^{5} v-36 x^{2} y^{7} v+y^{9} v$

$x^{9}-36 x^{7} y^{2}+126 x^{5} y^{4}-84 x^{3} y^{6}+9 x y^{8}+x^{9} v-36 x^{7} y^{2} v+126 x^{5} y^{4} v-84 x^{3} y^{6} v+9 x y^{8} v$

\section{$35^{\mathrm{a}}$ coluna:}

$-x^{9}+36 x^{7} y^{2}-126 x^{5} y^{4}+84 x^{3} y^{6}-9 x y^{8}-x^{9} v+36 x^{7} y^{2} v-126 x^{5} y^{4} v+84 x^{3} y^{6} v-9 x y^{8} v$

$9 x^{8} y-84 x^{6} y^{3}+126 x^{4} y^{5}-36 x^{2} y^{7}+y^{9}+9 x^{8} y v-84 x^{6} y^{3} v+126 x^{4} y^{5} v-36 x^{2} y^{7} v+y^{9} v$

$36^{a}$ coluna:

$50 x^{9} y-120 x^{7} y^{3}-756 x^{5} y^{5}+840 x^{3} y^{7}-110 x y^{9}+90 x^{9} y v-600 x^{7} y^{3} v+252 x^{5} y^{5} v+360 x^{3} y^{7} v-70 x y^{9} v$

$13 x^{10}-405 x^{8} y^{2}+1050 x^{6} y^{4}-210 x^{4} y^{6}-135 x^{2} y^{8}+7 y^{10}+9 x^{10} v-225 x^{8} y^{2} v+210 x^{6} y^{4} v+630 x^{4} y^{6} v-315 x^{2} y^{8} v+11 y^{10} v$

$37^{\mathrm{a}}$ coluna:

$-7 \mathrm{x}^{10}+135 \mathrm{x}^{8} \mathrm{y}^{2}+210 \mathrm{x}^{6} \mathrm{y}^{4}-1050 \mathrm{x}^{4} \mathrm{y}^{6}+405 \mathrm{x}^{2} \mathrm{y}^{8}-13 \mathrm{y}^{10}-11 \mathrm{x}^{10} v+315 \mathrm{x}^{8} \mathrm{y}^{2} v-630 \mathrm{x}^{6} \mathrm{y}^{4} v-210 \mathrm{x}^{4} \mathrm{y}^{6} v+225 \mathrm{x}^{2} \mathrm{y}^{8} v-9 \mathrm{y}^{10} v$ $110 x^{9} y-840 x^{7} y^{3}+756 x^{5} y^{5}+120 x^{3} y^{7}-50 x y^{9}+70 x^{9} y v-360 x^{7} y^{3} v-252 x^{5} y^{5} v+600 x^{3} y^{7} v-90 x y^{9} v$ 
$38^{\text {a }}$ coluna:

$10 x^{9} y-120 x^{7} y^{3}+252 x^{5} y^{5}-120 x^{3} y^{7}+10 x y^{9}+10 x^{9} y v-120 x^{7} y^{3} v+252 x^{5} y^{5} v-120 x^{3} y^{7} v+10 x y^{9} v$

$x^{10}-45 x^{8} y^{2}+210 x^{6} y^{4}-210 x^{4} y^{6}+45 x^{2} y^{8}-y^{10}+x^{10} v-45 x^{8} y^{2} v+210 x^{6} y^{4} v-210 x^{4} y^{6} v+45 x^{2} y^{8} v-y^{10} v$

39 coluna:

$-x^{10}+45 x^{8} y^{2}-210 x^{6} y^{4}+210 x^{4} y^{6}-45 x^{2} y^{8}+y^{10}-x^{10} v+45 x^{8} y^{2} v-210 x^{6} y^{4} v+210 x^{4} y^{6} v-45 x^{2} y^{8} v+y^{10} v$

$10 x^{9} y-120 x^{7} y^{3}+252 x^{5} y^{5}-120 x^{3} y^{7}+10 x y^{9}+10 x^{9} y v-120 x^{7} y^{3} v+252 x^{5} y^{5} v-120 x^{3} y^{7} v+10 x y^{9} v$

$40^{\mathrm{a}}$ coluna:

$66 x^{10} y-330 x^{8} y^{3}-924 x^{6} y^{5}+1980 x^{4} y^{7}-550 x^{2} y^{9}+14 y^{11}+110 x^{10} y v-990 x^{8} y^{3} v+924 x^{6} y^{5} v+660 x^{4} y^{7} v-330 x^{2} y^{9} v+10 y^{11} v$ $14 x^{11}-550 x^{9} y^{2}+1980 x^{7} y^{4}-924 x^{5} y^{6}-330 x^{3} y^{8}+66 x y^{10}+10 x^{11} v-330 x^{9} y^{2} v+660 x^{7} y^{4} v+924 x^{5} y^{6} v-990 x^{3} y^{8} v+110 x y^{10} v$

\section{$41^{\mathrm{a}}$ coluna:}

$-8 \mathrm{x}^{11}+220 \mathrm{x}^{9} \mathrm{y}^{2}-1848 \mathrm{x}^{5} \mathrm{y}^{6}+1320 \mathrm{x}^{3} \mathrm{y}^{8}-132 \mathrm{x} \mathrm{y}^{10}-12 \mathrm{x}^{11} v+440 \mathrm{x}^{9} \mathrm{y}^{2} v-1320 \mathrm{x}^{7} \mathrm{y}^{4} v+660 \mathrm{x}^{3} \mathrm{y}^{8} v-88 \mathrm{x} \mathrm{y}^{10} v$ $132 x^{10} y-1320 x^{8} y^{3}+1848 x^{6} y^{5}-220 x^{2} y^{9}+8 y^{11}+88 x^{10} y v-660 x^{8} y^{3} v+1320 x^{4} y^{7} v-440 x^{2} y^{9} v+12 y^{11} v$

\section{2 $2^{\mathrm{a}}$ coluna:}

$11 x^{10} y-165 x^{8} y^{3}+462 x^{6} y^{5}-330 x^{4} y^{7}+55 x^{2} y^{9}-y^{11}+11 x^{10} y v-165 x^{8} y^{3} v+462 x^{6} y^{5} v-330 x^{4} y^{7} v+55 x^{2} y^{9} v-y^{11} v$ $x^{11}-55 x^{9} y^{2}+330 x^{7} y^{4}-462 x^{5} y^{6}+165 x^{3} y^{8}-11 x y^{10}+x^{11} v-55 x^{9} y^{2} v+330 x^{7} y^{4} v-462 x^{5} y^{6} v+165 x^{3} y^{8} v-11 x y^{10} v$

\section{$43^{\text {a }}$ coluna:}

$-x^{11}+55 x^{9} y^{2}-330 x^{7} y^{4}+462 x^{5} y^{6}-165 x^{3} y^{8}+11 x y^{10}-x^{11} v+55 x^{9} y^{2} v-330 x^{7} y^{4} v+462 x^{5} y^{6} v-165 x^{3} y^{8} v+11 x y^{10} v$ $11 x^{10} y-165 x^{8} y^{3}+462 x^{6} y^{5}-330 x^{4} y^{7}+55 x^{2} y^{9}-y^{11}+11 x^{10} y v-165 x^{8} y^{3} v+462 x^{6} y^{5} v-330 x^{4} y^{7} v+55 x^{2} y^{9} v-y^{11} v$ 
$44^{a}$ coluna:

$84 x^{11} y-660 x^{9} y^{3}-792 x^{7} y^{5}+3960 x^{5} y^{7}-1980 x^{3} y^{9}+156 x y^{11}+132 x^{11} y v-1540 x^{9} y^{3} v+2376 x^{7} y^{5} v+792 x^{5} y^{7} v-1100 x^{3} y^{9} v+108 x y^{11} v$ $15 x^{12}-726 x^{10} y^{2}+3465 x^{8} y^{4}-2772 x^{6} y^{6}-495 x^{4} y^{8}+330 x^{2} y^{10}-9 y^{12}+11 x^{12} v-462 x^{10} y^{2} v+1485 x^{8} y^{4} v+924 x^{6} y^{6} v-2475 x^{4} y^{8} v+594 x^{2} y^{10} v-13 y^{12} v$

$45^{\mathrm{a}}$ coluna:

$-9 x^{12}+330 x^{10} y^{2}-495 x^{8} y^{4}-2772 x^{6} y^{6}+3465 x^{4} y^{8}-726 x^{2} y^{10}+15 y^{12}-13 x^{12} v+594 x^{10} y^{2} v-2475 x^{8} y^{4} v+924 x^{6} y^{6} v+1485 x^{4} y^{8} v-462 x^{2} y^{10} v+11 y^{12} v$ $156 x^{11} y-1980 x^{9} y^{3}+3960 x^{7} y^{5}-792 x^{5} y^{7}-660 x^{3} y^{9}+84 x y^{11}+108 x^{11} y v-1100 x^{9} y^{3} v+792 x^{7} y^{5} v+2376 x^{5} y^{7} v-1540 x^{3} y^{9} v+132 x y^{11} v$

$46^{\mathrm{a}}$ coluna:

$12 x^{11} y-220 x^{9} y^{3}+792 x^{7} y^{5}-792 x^{5} y^{7}+220 x^{3} y^{9}-12 x y^{11}+12 x^{11} y v-220 x^{9} y^{3} v+792 x^{7} y^{5} v-792 x^{5} y^{7} v+220 x^{3} y^{9} v-12 x y^{11} v$ $x^{12}-66 x^{10} y^{2}+495 x^{8} y^{4}-924 x^{6} y^{6}+495 x^{4} y^{8}-66 x^{2} y^{10}+y^{12}+x^{12} v-66 x^{10} y^{2} v+495 x^{8} y^{4} v-924 x^{6} y^{6} v+495 x^{4} y^{8} v-66 x^{2} y^{10} v+y^{12} v$

$47^{\mathrm{a}}$ coluna:

$-x^{12}+66 x^{10} y^{2}-495 x^{8} y^{4}+924 x^{6} y^{6}-495 x^{4} y^{8}+66 x^{2} y^{10}-y^{12}-x^{12} v+66 x^{10} y^{2} v-495 x^{8} y^{4} v+924 x^{6} y^{6} v-495 x^{4} y^{8} v+66 x^{2} y^{10} v-y^{12} v$

$12 x^{11} y-220 x^{9} y^{3}+792 x^{7} y^{5}-792 x^{5} y^{7}+220 x^{3} y^{9}-12 x y^{11}+12 x^{11} y v-220 x^{9} y^{3} v+792 x^{7} y^{5} v-792 x^{5} y^{7} v+220 x^{3} y^{9} v-12 x y^{11} v$

\section{- Matriz $\mathrm{S}_{\mathrm{c}}: \mathrm{S}_{\mathrm{c}}=\mathrm{DL}_{\mathrm{c}}{ }^{\top} \mathrm{N}_{\mathrm{c}}$}

Da $1^{\mathrm{a}}$ à $16^{\mathrm{a}}$ coluna:

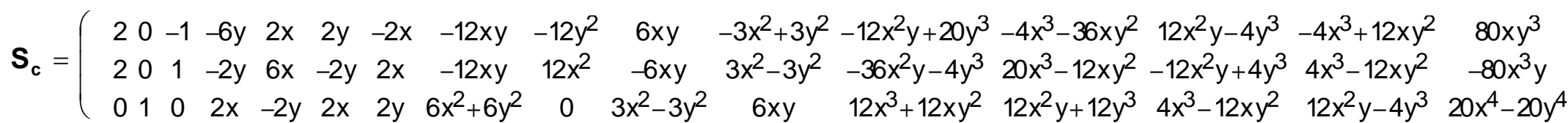


Da $17^{\mathrm{a}}$ à $22^{\mathrm{a}}$ coluna:

$\begin{array}{cccccc}-10 x^{4}-60 x^{2} y^{2}+30 y^{4} & 20 x^{3} y-20 x y^{3} & -5 x^{4}+30 x^{2} y^{2}-5 y^{4} & 30 x^{4} y+180 x^{2} y^{3}-42 y^{5} & -18 x^{5}-60 x^{3} y^{2}+150 x y^{4} & 30 x^{4} y-60 x^{2} y^{3}+6 y^{5} \\ 30 x^{4}-60 x^{2} y^{2}-10 y^{4} & -20 x^{3} y+20 x y^{3} & 5 x^{4}-30 x^{2} y^{2}+5 y^{4} & -150 x^{4} y+60 x^{2} y^{3}+18 y^{5} & 42 x^{5}-180 x^{3} y^{2}-30 x y^{4} & -30 x^{4} y+60 x^{2} y^{3}-6 y^{5}\end{array}$

$40 x^{3} y+40 x y^{3} \quad 5 x^{4}-30 x^{2} y^{2}+5 y^{4} \quad 20 x^{3} y-20 x y^{3} \quad 30 x^{5}-60 x^{3} y^{2}-90 x y^{4} \quad 90 x^{4} y+60 x^{2} y^{3}-30 y^{5} \quad 6 x^{5}-60 x^{3} y^{2}+30 x y^{4}$

Da $23^{\mathrm{a}}$ à $27^{\mathrm{a}}$ coluna:

$-6 x^{5}+60 x^{3} y^{2}-30 x y^{4} \quad 84 x^{5} y+280 x^{3} y^{3}-252 x y^{5} \quad-28 x^{6}+420 x^{2} y^{4}-56 y^{6} \quad 42 x^{5} y-140 x^{3} y^{3}+42 x y^{5} \quad-7 x^{6}+105 x^{4} y^{2}-105 x^{2} y^{4}+7 y^{6}$ $6 x^{5}-60 x^{3} y^{2}+30 x y^{4} \quad-252 x^{5} y+280 x^{3} y^{3}+84 x y^{5} \quad 56 x^{6}-420 x^{4} y^{2}+28 y^{6} \quad-42 x^{5} y+140 x^{3} y^{3}-42 x y^{5} \quad 7 x^{6}-105 x^{4} y^{2}+105 x^{2} y^{4}-7 y^{6}$ $30 x^{4} y-60 x^{2} y^{3}+6 y^{5} \quad 42 x^{6}-210 x^{4} y^{2}-210 x^{2} y^{4}+42 y^{6} \quad 168 x^{5} y-168 x y^{5} \quad 7 x^{6}-105 x^{4} y^{2}+105 x^{2} y^{4}-7 y^{6} \quad 42 x^{5} y-140 x^{3} y^{3}+42 x y^{5}$

Da $28^{\mathrm{a}}$ à $31^{\mathrm{a}}$ coluna:

$168 x^{6} y+280 x^{4} y^{3}-840 x^{2} y^{5}+72 y^{7}-40 x^{7}+168 x^{5} y^{2}+840 x^{3} y^{4}-392 x y^{6} \quad 56 x^{6} y-280 x^{4} y^{3}+168 x^{2} y^{5}-8 y^{7}-8 x^{7}+168 x^{5} y^{2}-280 x^{3} y^{4}+56 x y^{6}$ $-392 x^{6} y+840 x^{4} y^{3}+168 x^{2} y^{5}-40 y^{7} \quad 72 x^{7}-840 x^{5} y^{2}+280 x^{3} y^{4}+168 x y^{6}-56 x^{6} y+280 x^{4} y^{3}-168 x^{2} y^{5}+8 y^{7} \quad 8 x^{7}-168 x^{5} y^{2}+280 x^{3} y^{4}-56 x y^{6}$ $56 x^{7}-504 x^{5} y^{2}-280 x^{3} y^{4}+280 x y^{6} \quad 280 x^{6} y-280 x^{4} y^{3}-504 x^{2} y^{5}+56 y^{7} \quad 8 x^{7}-168 x^{5} y^{2}+280 x^{3} y^{4}-56 x y^{6} \quad 56 x^{6} y-280 x^{4} y^{3}+168 x^{2} y^{5}-8 y^{7}$

Da $32^{\mathrm{a}}$ à $35^{\mathrm{a}}$ coluna:

$288 x^{7} y-2016 x^{3} y^{5}+576 x y^{7} \quad-54 x^{8}+504 x^{6} y^{2}+1260 x^{4} y^{4}-1512 x^{2} y^{6}+90 y^{8} \quad 72 x^{7} y-504 x^{5} y^{3}+504 x^{3} y^{5}-72 x y^{7} \quad-9 x^{8}+252 x^{6} y^{2}-630 x^{4} y^{4}+252 x^{2} y^{6}-9 y^{8} \quad .$. $-576 x^{7} y+2016 x^{5} y^{3}-288 x y^{7} \quad 90 x^{8}-1512 x^{6} y^{2}+1260 x^{4} y^{4}+504 x^{2} y^{6}-54 y^{8} \quad-72 x^{7} y+504 x^{5} y^{3}-504 x^{3} y^{5}+72 x y^{7} \quad 9 x^{8}-252 x^{6} y^{2}+630 x^{4} y^{4}-252 x^{2} y^{6}+9 y^{8}$ $72 x^{8}-1008 x^{6} y^{2}+1008 x^{2} y^{6}-72 y^{8} \quad 432 x^{7} y-1008 x^{5} y^{3}-1008 x^{3} y^{5}+432 x y^{7} \quad 9 x^{8}-252 x^{6} y^{2}+630 x^{4} y^{4}-252 x^{2} y^{6}+9 y^{8} \quad 72 x^{7} y-504 x^{5} y^{3}+504 x^{3} y^{5}-72 x y^{7}$

\section{Da $36^{\mathrm{a}}$ à $38^{\mathrm{a}}$ coluna:}

$450 x^{8} y-840 x^{6} y^{3}-3780 x^{4} y^{5}+2520 x^{2} y^{7}-110 y^{9}-70 x^{9}+1080 x^{7} y^{2}+1260 x^{5} y^{4}-4200 x^{3} y^{6}+810 x y^{8} 90 x^{8} y-840 x^{6} y^{3}+1260 x^{4} y^{5}-360 x^{2} y^{7}+10 y^{9}$ $-810 x^{8} y+4200 x^{6} y^{3}-1260 x^{4} y^{5}-1080 x^{2} y^{7}+70 y^{9} \quad 110 x^{9}-2520 x^{7} y^{2}+3780 x^{5} y^{4}+840 x^{3} y^{6}-450 x y^{8}-90 x^{8} y+840 x^{6} y^{3}-1260 x^{4} y^{5}+360 x^{2} y^{7}-10 y^{9}$ $90 x^{9}-1800 x^{7} y^{2}+1260 x^{5} y^{4}+2520 x^{3} y^{6}-630 x y^{8} \quad 630 x^{8} y-2520 x^{6} y^{3}-1260 x^{4} y^{5}+1800 x^{2} y^{7}-90 y^{9} \quad 10 x^{9}-360 x^{7} y^{2}+1260 x^{5} y^{4}-840 x^{3} y^{6}+90 x y^{8}$ 
Da $39^{\mathrm{a}}$ à $41^{\mathrm{a}}$ coluna:
$-10 x^{9}+360 x^{7} y^{2}-1260 x^{5} y^{4}+840 x^{3} y^{6}-90 x y^{8}$ $10 x^{9}-360 x^{7} y^{2}+1260 x^{5} y^{4}-840 x^{3} y^{6}+90 x y^{8}$
$660 x^{9} y-2640 x^{7} y^{3}-5544 x^{5} y^{5}+7920 x^{3} y^{7}-1100 x y^{9}$ $-1100 x^{9} y+7920 x^{7} y^{3}-5544 x^{5} y^{5}-2640 x^{3} y^{7}+660 x y^{9}$
$-88 x^{10}+1980 x^{8} y^{2}-9240 x^{4} y^{6}+3960 x^{2} y^{8}-132 y^{10}$ $132 x^{10}-3960 x^{8} y^{2}+9240 x^{6} y^{4}-1980 x^{2} y^{8}+88 y^{10}$ $90 x^{8} y-840 x^{6} y^{3}+1260 x^{4} y^{5}-360 x^{2} y^{7}+10 y^{9} \quad 110 x^{10}-2970 x^{8} y^{2}+4620 x^{6} y^{4}+4620 x^{4} y^{6}-2970 x^{2} y^{8}+110 y^{10}$ $880 x^{9} y-5280 x^{7} y^{3}+5280 x^{3} y^{7}-880 x y^{9}$

$42^{\mathrm{a}}$ e $43^{\mathrm{a}}$ colunas:

$110 x^{9} y-1320 x^{7} y^{3}+272 x^{5} y^{5}-1320 x^{3} y^{7}+110 x y^{9} \quad-11 x^{10}+495 x^{8} y^{2}-2310 x^{6} y^{4}+2310 x^{4} y^{6}-495 x^{2} y^{8}+11 y^{10}$ $-110 x^{9} y+1320 x^{7} y^{3}-2772 x^{5} y^{5}+1320 x^{3} y^{7}-110 x y^{9} \quad 11 x^{10}-495 x^{8} y^{2}+2310 x^{6} y^{4}-2310 x^{4} y^{6}+495 x^{2} y^{8}-11 y^{10}$ $11 x^{10}-495 x^{8} y^{2}+2310 x^{6} y^{4}-2310 x^{4} y^{6}+495 x^{2} y^{8}-11 y^{10} \quad 110 x^{9} y-1320 x^{7} y^{3}+2772 x^{5} y^{5}-1320 x^{3} y^{7}+110 x y^{9}$

$44^{\mathrm{a}}$ e $45^{\mathrm{a}}$ colunas:

$924 x^{10} y-5940 x^{8} y^{3}-5544 x^{6} y^{5}+19800 x^{4} y^{7}-5940 x^{2} y^{9}+156 y^{11}-108 x^{11}+3300 x^{9} y^{2}-3960 x^{7} y^{4}-16632 x^{5} y^{6}+13860 x^{3} y^{8}-1452 x y^{10}$ $-1452 x^{10} y+13860 x^{8} y^{3}-16632 x^{6} y^{5}-3960 x^{4} y^{7}+3300 x^{2} y^{9}-108 y^{11} \quad 156 x^{11}-5940 x^{9} y^{2}+19800 x^{7} y^{4}-5544 x^{5} y^{6}-5940 x^{3} y^{8}+924 x y^{10}$ $132 x^{11}-4620 x^{9} y^{2}+11880 x^{7} y^{4}+5544 x^{5} y^{6}-9900 x^{3} y^{8}+1188 x y^{10} \quad 1188 x^{10} y-9900 x^{8} y^{3}+5544 x^{6} y^{5}+11880 x^{4} y^{7}-4620 x^{2} y^{9}+132 y^{11}$

$46^{\mathrm{a}}$ e $47^{\mathrm{a}}$ colunas:

$132 x^{10} y-1980 x^{8} y^{3}+5544 x^{6} y^{5}-3960 x^{4} y^{7}+660 x^{2} y^{9}-12 y^{11}-12 x^{11}+660 x^{9} y^{2}-3960 x^{7} y^{4}+5544 x^{5} y^{6}-1980 x^{3} y^{8}+132 x y^{10}$ $-132 x^{10} y+1980 x^{8} y^{3}-5544 x^{6} y^{5}+3960 x^{4} y^{7}-660 x^{2} y^{9}+12 y^{11} 12 x^{11}-660 x^{9} y^{2}+3960 x^{7} y^{4}-5544 x^{5} y^{6}+1980 x^{3} y^{8}-132 x y^{10}$ $\left.12 x^{11}-660 x^{9} y^{2}+3960 x^{7} y^{4}-5544 x^{5} y^{6}+1980 x^{3} y^{8}-132 x y^{10} \quad 132 x^{10} y-1980 x^{8} y^{3}+5544 x^{6} y^{5}-3960 x^{4} y^{7}+660 x^{2} y^{9}-12 y^{11}\right)$ 


\section{Apêndice B - Matrizes utilizadas na aproximação dos campos de deslocamentos e tensões em domínios com orifício circular: construção a partir da equação de Navier}

As matrizes apresentadas a seguir foram obtidas com a utilização do software Mathematica ${ }^{\circledR}$.

- Matriz $\mathrm{N}_{\mathrm{p}}$ :

Da $1^{\mathrm{a}}$ à $3^{\mathrm{a}}$ coluna:

$$
\mathbf{N}_{\mathbf{p}}=\left(\begin{array}{ccc}
\frac{-r^{2}(-1+v)+a^{2}(1+v)}{r-r v} & \left(r^{2}-\frac{a^{4}(1+v)}{r^{2}(-1+3 v)}\right) \cos \theta & \left(r^{2}-\frac{a^{4}(1+v)}{r^{2}(-1+3 v)}\right) \operatorname{sen} \theta \\
0 & -\left(\frac{a^{4}(1+v)+r^{4}(5+v)}{r^{2}(-1+3 v)}\right) \operatorname{sen} \theta & \left(\frac{a^{4}(1+v)+r^{4}(5+v)}{r^{2}(-1+3 v)}\right) \cos \theta
\end{array}\right.
$$

$4^{\mathrm{a}}$ e $5^{\mathrm{a}}$ colunas:

$$
\begin{array}{cc}
\left(\frac{4\left(a^{4}-r^{4} v+a^{2} r^{2}(1+v)\right)}{r}\right) \cos 2 \theta & \left(\frac{1}{r^{3}}-\frac{r}{a^{4}}-\frac{4}{a^{2} r(1+v)}\right) \cos 2 \theta \\
\left(\frac{2(a-r)(a+r)\left(a^{2}(-1+v)-r^{2}(3+v)\right)}{r}\right) \operatorname{sen} 2 \theta & \left(\frac{\left(a^{2}+r^{2}\right)^{2}+\left(a^{2}-r^{2}\right)^{2} v}{a^{4} r^{3}(1+v)}\right) \operatorname{sen} 2 \theta
\end{array}
$$

$6^{\mathrm{a}}$ e $7^{\mathrm{a}}$ colunas:

$$
\begin{array}{cc}
\left(\frac{4\left(a^{4}-r^{4} v+a^{2} r^{2}(1+v)\right)}{r}\right) \operatorname{sen} 2 \theta & \left(\frac{1}{r^{3}}-\frac{r}{a^{4}}-\frac{4}{a^{2} r(1+v)}\right) \operatorname{sen} 2 \theta \\
-\left(\frac{2(a-r)(a+r)\left(a^{2}(-1+v)-r^{2}(3+v)\right)}{r}\right) \cos 2 \theta & -\left(\frac{\left(a^{2}+r^{2}\right)^{2}+\left(a^{2}-r^{2}\right)^{2} v}{a^{4} r^{3}(1+v)}\right) \cos 2 \theta
\end{array}
$$


Da $8^{a}$ coluna em diante $(k \geq 3)$ :

$$
\begin{array}{llll}
\mathbf{N}_{\mathrm{Ap}} & \mathbf{N}_{\mathrm{Bp}} & \mathbf{N}_{\mathrm{Cp}} & \left.\mathbf{N}_{\mathrm{Dp}} \cdots\right)
\end{array}
$$

onde:

$$
\begin{aligned}
& \mathbf{N}_{\text {Ap }}=\left(\begin{array}{c}
\left(\frac{r^{-1-k}\left(-a^{2 k} r^{2}(2+k+(-2+k) v)+r^{2 k}\left(-a^{2} k^{2}(1+v)+(-1+k) r^{2}(-2+k+(2+k) v)\right)\right)}{(-1+k)(-2+k+(2+k) v)}\right) \cos k \theta \\
\left(\frac{r^{-1-k}\left(-a^{2 k} r^{2}(-4+k+k v)+r^{2 k}\left(a^{2} k^{2}(1+v)-(-1+k) r^{2}(4+k+k v)\right)\right)}{(-1+k)(-2+k+(2+k) v)}\right) \operatorname{sen} k \theta
\end{array}\right) \\
& \mathbf{N}_{\mathbf{B p}}=\left(\begin{array}{c}
\left(\frac{\mathrm{a}^{-2(1+k)} \mathrm{r}^{-1-k}\left(-\mathrm{a}^{2} \mathrm{r}^{2 \mathrm{k}}(1+v)+\mathrm{a}^{2 \mathrm{k}}\left(\mathrm{a}^{2}(-1+\mathrm{k})(1+v)-\mathrm{r}^{2}(2+\mathrm{k}+(-2+\mathrm{k}) v)\right)\right)}{(-1+\mathrm{k})(1+v)}\right) \cos \mathrm{k} \theta \\
\left(\frac{\mathrm{a}^{-2(1+k)} \mathrm{r}^{-1-k}\left(\mathrm{a}^{2} \mathrm{r}^{2 \mathrm{k}}(1+v)+\mathrm{a}^{2 \mathrm{k}}\left(\mathrm{a}^{2}(-1+\mathrm{k})(1+v)-\mathrm{r}^{2}(-4+\mathrm{k}+\mathrm{k} v)\right)\right)}{(-1+\mathrm{k})(1+v)}\right) \operatorname{sen} \mathrm{\theta} \theta
\end{array}\right) \\
& \mathbf{N}_{\mathrm{Cp}}=\left(\begin{array}{c}
\left(\frac{\mathrm{r}^{-1-\mathrm{k}}\left(-\mathrm{a}^{2 \mathrm{k}} \mathrm{r}^{2}(2+\mathrm{k}+(-2+\mathrm{k}) v)+\mathrm{r}^{2 \mathrm{k}}\left(-\mathrm{a}^{2} \mathrm{k}^{2}(1+v)+(-1+\mathrm{k}) \mathrm{r}^{2}(-2+\mathrm{k}+(2+\mathrm{k}) v)\right)\right)}{(-1+\mathrm{k})(-2+\mathrm{k}+(2+\mathrm{k}) v)}\right) \operatorname{sen} \mathrm{k} \theta \\
\left(-\frac{\mathrm{r}^{-1-\mathrm{k}}\left(-\mathrm{a}^{2 \mathrm{k}} \mathrm{r}^{2}(-4+\mathrm{k}+\mathrm{k} v)+\mathrm{r}^{2 \mathrm{k}}\left(\mathrm{a}^{2} \mathrm{k}^{2}(1+v)-(-1+\mathrm{k}) \mathrm{r}^{2}(4+\mathrm{k}+\mathrm{k} v)\right)\right)}{(-1+\mathrm{k})(-2+\mathrm{k}+(2+\mathrm{k}) v)}\right) \cos \mathrm{k} \theta
\end{array}\right) \\
& \mathbf{N}_{D p}=\left(\begin{array}{c}
\left(\frac{a^{-2(1+k)} r^{-1-k}\left(-a^{2} r^{2 k}(1+v)+a^{2 k}\left(a^{2}(-1+k)(1+v)-r^{2}(2+k+(-2+k) v)\right)\right)}{(-1+k)(1+v)}\right) \operatorname{sen} k \theta \\
\left(-\frac{a^{-2(1+k)} r^{-1-k}\left(a^{2} r^{2 k}(1+v)+a^{2 k}\left(a^{2}(-1+k)(1+v)-r^{2}(-4+k+k v)\right)\right)}{(-1+k)(1+v)}\right) \cos k \theta
\end{array}\right)
\end{aligned}
$$

- Matriz $S_{p}: S_{p}=D M_{\varepsilon} N_{p}+D L_{p}^{\top} N_{p}$

Da $1^{\mathrm{a}}$ à $4^{\mathrm{a}}$ coluna:

$$
\mathbf{S}_{\mathbf{p}}=E\left(\begin{array}{cccc}
\frac{a^{2}-r^{2}}{r^{2}(-1+v)} & \left(\frac{2\left(a^{4}-r^{4}\right)}{r^{3}(-1+3 v)}\right) \cos \theta & \left(\frac{2\left(a^{4}-r^{4}\right)}{r^{3}(-1+3 v)}\right) \operatorname{sen} \theta & \left(\frac{4 a^{2}\left(-a^{2}+r^{2}\right)}{r^{2}}\right) \cos 2 \theta \\
-\frac{a^{2}+r^{2}}{r^{2}(-1+v)} & \left(-\frac{2\left(a^{4}+3 r^{4}\right)}{r^{3}(-1+3 v)}\right) \cos \theta & \left(-\frac{2\left(a^{4}+3 r^{4}\right)}{r^{3}(-1+3 v)}\right) \operatorname{sen} \theta & \left(-4\left(a^{2}-3 r^{2}\right)\right) \cos 2 \theta \\
0 & \left(\frac{2\left(a^{4}-r^{4}\right)}{r^{3}(-1+3 v)}\right) \operatorname{sen} \theta & \left(-\frac{2\left(a^{4}-r^{4}\right)}{r^{3}(-1+3 v)}\right) \cos \theta & \left(-\frac{2\left(a^{4}+2 a^{2} r^{2}-3 r^{4}\right)}{r^{2}}\right) \operatorname{sen} 2 \theta
\end{array} .\right.
$$


Da $5^{\mathrm{a}}$ à $7^{\mathrm{a}}$ coluna:

$$
\begin{array}{ccc}
\left(\begin{array}{ccc}
\left.-\frac{3 a^{4}-4 a^{2} r^{2}+r^{4}}{a^{4} r^{4}(1+v)}\right) \cos 2 \theta & \left(\frac{4 a^{2}\left(-a^{2}+r^{2}\right)}{r^{2}}\right) \operatorname{sen} 2 \theta & \left(-\frac{3 a^{4}-4 a^{2} r^{2}+r^{4}}{a^{4} r^{4}(1+v)}\right) \operatorname{sen} 2 \theta \\
\left(\frac{3 a^{4}+r^{4}}{a^{4} r^{4}(1+v)}\right) \cos 2 \theta & \left(-4\left(a^{2}-3 r^{2}\right)\right) \operatorname{sen} 2 \theta & \left(\frac{3 a^{4}+r^{4}}{a^{4} r^{4}(1+v)}\right) \operatorname{sen} 2 \theta
\end{array}\right] \\
\left(-\frac{3 a^{4}-2 a^{2} r^{2}-r^{4}}{a^{4} r^{4}(1+v)}\right) \operatorname{sen} 2 \theta & \left(\frac{2\left(a^{4}+2 a^{2} r^{2}-3 r^{4}\right)}{r^{2}}\right) \cos 2 \theta & \left(\frac{3 a^{4}-2 a^{2} r^{2}-r^{4}}{a^{4} r^{4}(1+v)}\right) \cos 2 \theta
\end{array}
$$

Da $8^{\mathrm{a}}$ coluna em diante $(\mathrm{k} \geq 3)$ :

$$
\begin{array}{llll}
\mathbf{S}_{A p} & \mathbf{S}_{B p} & \mathbf{S}_{\mathrm{Cp}} & \left.\mathbf{S}_{\mathrm{Dp}} \ldots\right)
\end{array}
$$

onde:

$$
S_{A p}=\left(\begin{array}{c}
\left(\frac{r^{-2(1+k)}\left(a^{2 k}(2+k) r^{2}-a^{2} k^{2} r^{2 k}+\left(-2-k+k^{2}\right) r^{2(1+k)}\right)}{-2+k+2 v+k v}\right) \cos k \theta \\
\left(-\frac{r^{-2(1+k)}\left(a^{2 k}(-2+k) r^{2}-a^{2} k^{2} r^{2 k}+\left(2+3 k+k^{2}\right) r^{2(1+k)}\right)}{-2+k+2 v+k v}\right) \cos k \theta \\
\left(\frac{k r^{-2(1+k)}\left(a^{2 k} r^{2}+a^{2} k r^{2 k}-(1+k) r^{2(1+k)}\right)}{-2+k+2 v+k v}\right) \operatorname{sen} k \theta
\end{array}\right)
$$

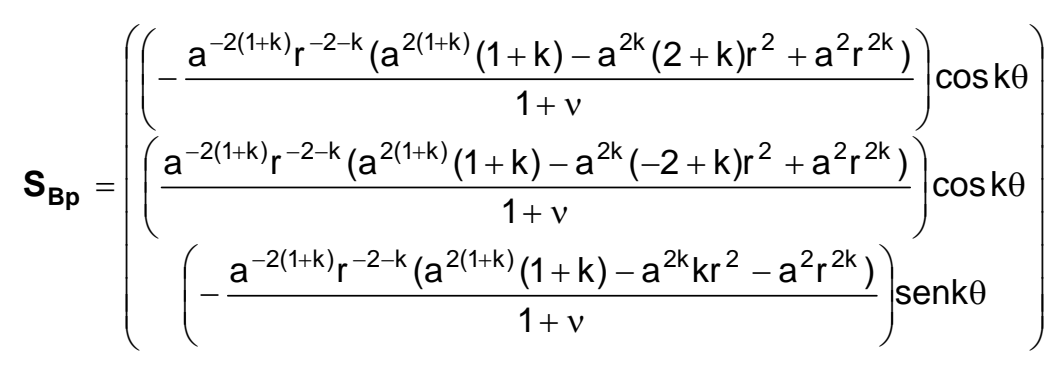

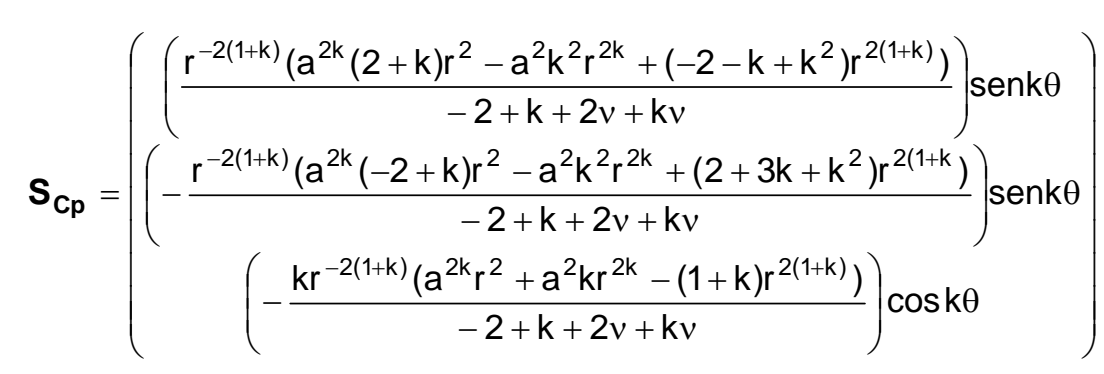

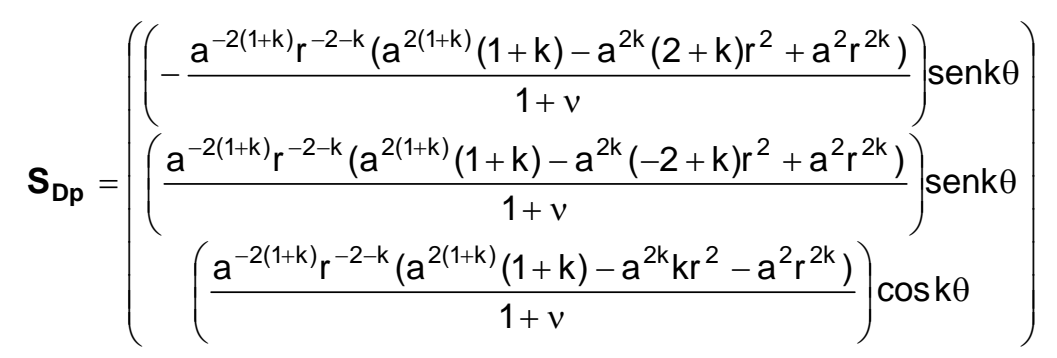




\section{Apêndice C - Exemplo de montagem do sistema resolutivo}

Com o intuito de elucidar o procedimento de montagem do sistema global e a verificação ao equilíbrio de forças nodais equivalentes, apresenta-se como exemplo uma chapa discretizada em dois elementos, conforme ilustrado na Figura C.1.

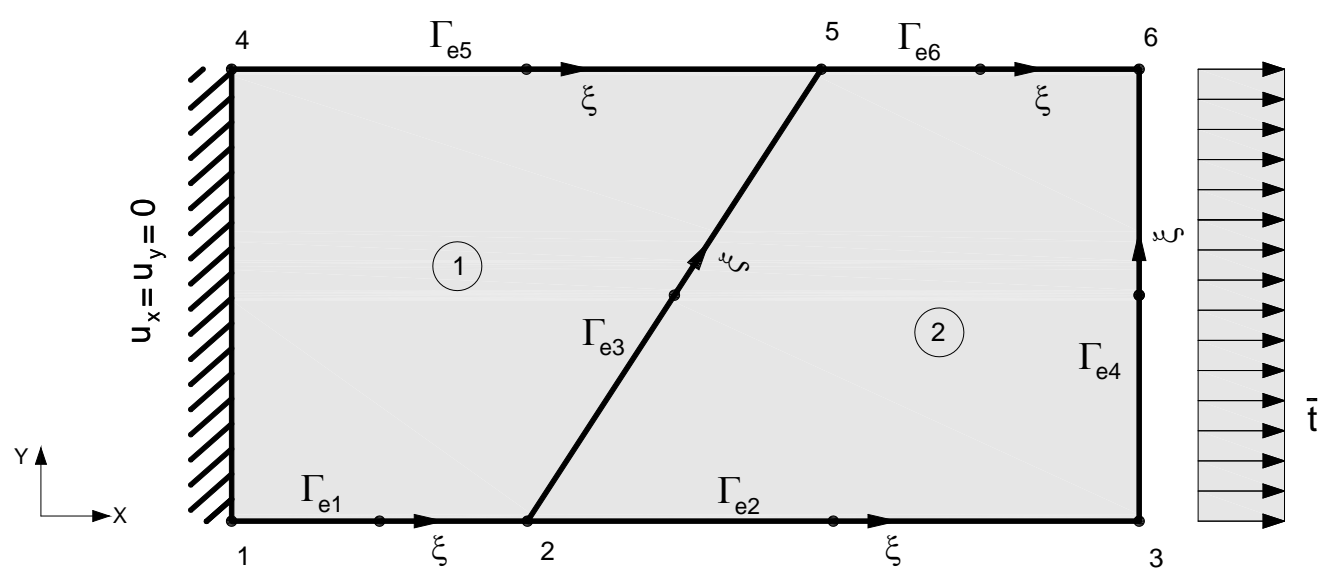

Figura C.1. Chapa discretizada em dois elementos.

Para esta discretização, o sistema global pode ser representado por:

$$
P X=Q
$$

sendo $P=\left(\begin{array}{ccc}F_{1} & 0 & -G_{1} \\ 0 & F_{2} & -G_{2} \\ -G_{1}{ }^{T} & -G_{2}{ }^{T} & 0\end{array}\right), X=\left\{\begin{array}{l}c_{1} \\ c_{2} \\ d_{t}\end{array}\right\}$ e $Q=\left\{\begin{array}{c}e_{1} \\ e_{2} \\ -q_{t}\end{array}\right\}$.

- Montagem

Na montagem da matriz $\boldsymbol{P}$, as matrizes $\boldsymbol{F}_{1}$ e $\boldsymbol{F}_{2}$ são alocadas de forma independe, já que os graus de liberdade em tensão são restritos ao domínio de cada elemento. Por outro lado, as matrizes $G_{1}$ e $G_{2}$ devem ser alocadas pelo compartilhamento dos graus de liberdade em deslocamentos do sistema, considerando-se cada trecho da rede.

Por exemplo, a parcela de $G_{1}$ referente ao trecho $\Gamma_{\mathrm{e} 1}$, pode ser escrita por:

$$
\left.\boldsymbol{G}_{1 \Gamma_{e 1}}=\int_{\Gamma_{e 1}} \boldsymbol{T}_{1}^{T} \tilde{\boldsymbol{U}} d \Gamma=\int_{\Gamma_{e 1}} \boldsymbol{T}_{1}^{T} \tilde{\boldsymbol{U}}_{\phi 1} \quad \boldsymbol{T}_{1}^{T} \tilde{\boldsymbol{U}}_{\phi 2}\right] d \Gamma=\left[\begin{array}{lll}
\boldsymbol{G}_{1 \Gamma_{e 1} 1} & \boldsymbol{G}_{1 \Gamma_{e 1}}{ }^{2}
\end{array}\right]
$$

onde os sobrescritos 1 e 2 das matrizes $G_{1 \Gamma_{e 1}}{ }^{1}$ e $G_{1 \Gamma_{e 1}}{ }^{2}$, indicam a posição de alocação de 
acordo com o respectivo nó.

Analogamente, para as outras situações, escreve-se:

Parcela de $G_{1}$ em $\Gamma_{e 3}$ :

$$
\left.\boldsymbol{G}_{1 \Gamma_{e 3}}=\int_{\Gamma_{e 3}} \boldsymbol{T}_{1}^{T} \tilde{\boldsymbol{U}} d \Gamma=\int_{\Gamma_{e 3}} \mid \boldsymbol{T}_{1}^{T} \tilde{\boldsymbol{U}}_{\phi 1} \quad \boldsymbol{T}_{1}^{T} \tilde{\boldsymbol{U}}_{\phi 2}\right\rfloor d \Gamma=\left[\begin{array}{ll}
\boldsymbol{G}_{1 \Gamma_{e 3}}{ }^{2} & \boldsymbol{G}_{1 \Gamma_{e 3}}{ }^{5}
\end{array}\right]
$$

Parcela de $G_{1}$ em $\Gamma_{e 5}$ :

$$
\left.\boldsymbol{G}_{1 \Gamma_{e 5}}=\int_{\Gamma_{e 5}} \boldsymbol{T}_{1}^{T} \tilde{\boldsymbol{U}} d \Gamma=\int_{\Gamma_{e 5}} \mid \boldsymbol{T}_{1}^{T} \tilde{\boldsymbol{U}}_{\phi 1} \quad \boldsymbol{T}_{1}^{T} \tilde{\boldsymbol{U}}_{\phi 2}\right] d \Gamma=\left[\begin{array}{ll}
\boldsymbol{G}_{1 \Gamma_{e 5}}{ }^{4} & \boldsymbol{G}_{1 \Gamma_{e 5}}
\end{array}\right]
$$

Parcela de $G_{2}$ em $\Gamma_{\mathrm{e} 2}$ :

$$
\left.\boldsymbol{G}_{2 \Gamma_{e 2}}=\int_{\Gamma_{e 2}} \boldsymbol{T}_{2}^{\top} \tilde{\boldsymbol{U}} d \Gamma=\int_{\Gamma_{e 2}} \mid \boldsymbol{T}_{2}^{\top} \tilde{\boldsymbol{U}}_{\phi 1} \quad \boldsymbol{T}_{2}^{\top} \tilde{\boldsymbol{U}}_{\phi 2}\right\rfloor d \Gamma=\left[\begin{array}{ll}
\boldsymbol{G}_{2 \Gamma_{e 2}}{ }^{2} & \boldsymbol{G}_{2 \Gamma_{e 2}}{ }^{3}
\end{array}\right]
$$

Parcela de $G_{2}$ em $\Gamma_{\text {eз }}$ :

$$
\boldsymbol{G}_{2 \Gamma_{e 3}}=\int_{\Gamma_{e 3}} \boldsymbol{T}_{2}^{\top} \tilde{\boldsymbol{U}} d \Gamma=\int_{\Gamma_{e 3}}\left|\boldsymbol{T}_{2}^{\top} \tilde{\boldsymbol{U}}_{\phi 1} \quad \boldsymbol{T}_{2}^{\top} \tilde{\boldsymbol{U}}_{\phi 2}\right| d \Gamma=\left[\begin{array}{ll}
\boldsymbol{G}_{2 \Gamma_{e 3}}{ }^{2} & \boldsymbol{G}_{2 \Gamma_{e 3}}{ }^{5}
\end{array}\right] ;
$$

Parcela de $G_{2}$ em $\Gamma_{\mathrm{e} 4}$ :

$$
\left.\boldsymbol{G}_{2 \Gamma_{e 4}}=\int_{\Gamma_{e 4}} \boldsymbol{T}_{2}^{T} \tilde{\boldsymbol{U}} d \Gamma=\int_{\Gamma_{e 4}} \mid \boldsymbol{T}_{2}^{T} \tilde{\boldsymbol{U}}_{\phi 1} \boldsymbol{T}_{2}^{T} \tilde{\boldsymbol{U}}_{\phi 2}\right\rfloor d \Gamma=\left[\begin{array}{ll}
\boldsymbol{G}_{2 \Gamma_{e 4}}{ }^{3} & \boldsymbol{G}_{2 \Gamma_{e 4}}{ }^{6}
\end{array}\right]
$$

Parcela de $G_{2}$ em $\Gamma_{\mathrm{e} 6}$ :

$$
\left.\boldsymbol{G}_{2 \Gamma_{e 6}}=\int_{\Gamma_{e 6}} \boldsymbol{T}_{2}^{T} \tilde{\boldsymbol{U}} d \Gamma=\int_{\Gamma_{e 6}} \boldsymbol{T}_{2}^{T} \tilde{\boldsymbol{U}}_{\phi 1} \boldsymbol{T}_{2}^{T} \tilde{\boldsymbol{U}}_{\phi 2}\right] d \Gamma=\left[\begin{array}{ll}
\boldsymbol{G}_{2 \Gamma_{e 6}}{ }^{5} & \boldsymbol{G}_{2 \Gamma_{e 6}}{ }^{6}
\end{array}\right]
$$

Da Eq. (C.2) até a (C.8) utilizou-se a notação genérica $\boldsymbol{T}_{\boldsymbol{1}}=\boldsymbol{A} \boldsymbol{S}_{\boldsymbol{1}}$ e $\boldsymbol{T}_{2}=\boldsymbol{A} \boldsymbol{S}_{2}$ para os elementos 1 e 2, respectivamente. Evidentemente $\boldsymbol{A}$ depende dos co-senos diretores do trecho considerado.

A partir das considerações anteriores, a matriz $\boldsymbol{P}$ fica com o aspecto indicado na Tabela $\mathrm{C} 1$. 
Tabela C.1 - Montagem da matriz dos coeficientes

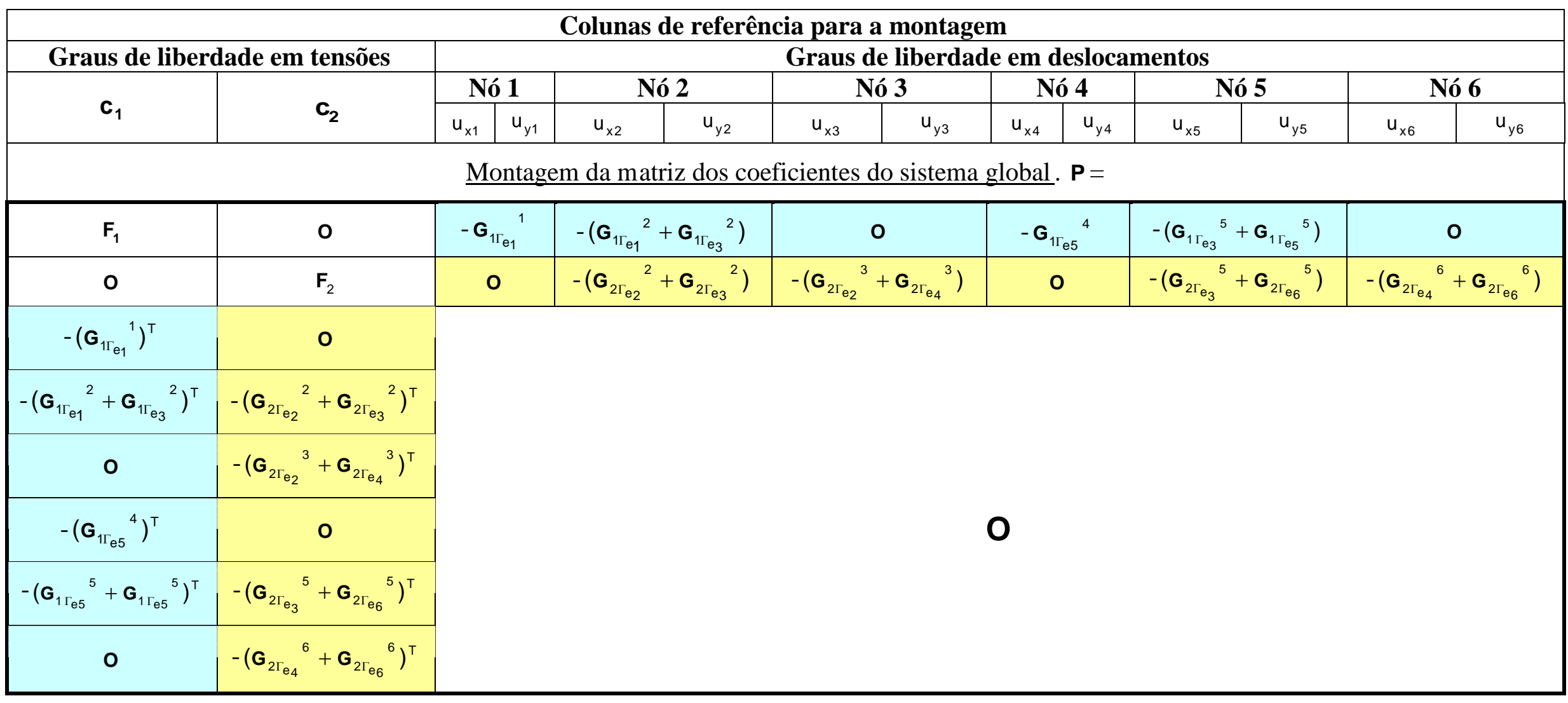

\begin{tabular}{|l|l|}
\hline & Matrizes : $-\mathbf{G}_{\mathbf{1}}$ e $-\mathbf{G}_{\mathbf{1}}{ }^{\top}$ \\
\hline & Matrizes: $-\mathbf{G}_{\mathbf{2}}$ e $-\mathbf{G}_{\mathbf{2}}{ }^{\top}$ \\
\hline
\end{tabular}


Na montagem do vetor $\boldsymbol{Q}$, deve-se proceder de forma análoga. Os vetores $\boldsymbol{e}_{1}$ e $\boldsymbol{e}_{2}$ são alocados de forma independentes. No exemplo considerado eles são nulos, pois nenhum deslocamento foi imposto à chapa. Por outro lado, o vetor de forças nodais equivalentes $\boldsymbol{q}_{\boldsymbol{t}}$ deve ser alocado pela contribuição de cada trecho conforme as posições dos nós.

Sendo $\Gamma_{\mathrm{e} 4} \mathrm{o}$ único trecho carregado, tem-se:

$$
\boldsymbol{q}_{\Gamma_{e 4}}=\int_{\Gamma_{e 4}} \tilde{\boldsymbol{U}}^{T} \overline{\boldsymbol{t}} d \Gamma=\int_{\Gamma_{e 4}}\left\{\begin{array}{l}
\tilde{\boldsymbol{U}}_{\phi 1} \overline{\boldsymbol{t}} \\
\tilde{\boldsymbol{U}}_{\phi 2} \overline{\boldsymbol{t}}
\end{array}\right\} d \Gamma=\left\{\begin{array}{l}
\boldsymbol{q}_{\Gamma_{e 4}}{ }^{3} \\
\boldsymbol{q}_{\Gamma_{e 4}}{ }^{6}
\end{array}\right\} ;
$$

onde os sobrescritos 3 e 6 dos vetores $\boldsymbol{q}_{\Gamma_{e 4}}{ }^{3}$ e $\boldsymbol{q}_{\Gamma_{e 4}}{ }^{6}$, indicam a posição de alocação de acordo com o respectivo nó.

Assim sendo, o vetor $\boldsymbol{Q}$ adquire a forma:

$$
\boldsymbol{Q}=\left\{\begin{array}{c}
\boldsymbol{e}_{1} \\
\boldsymbol{e}_{2} \\
-\boldsymbol{q}_{\boldsymbol{t}}
\end{array}\right\}=\left\{\begin{array}{c}
0 \\
0 \\
0 \\
0 \\
-\boldsymbol{q}_{\Gamma_{e 4}} 3 \\
\boldsymbol{0} \\
0 \\
-\boldsymbol{q}_{\Gamma_{e 4}} 6
\end{array}\right\}
$$

Por sua vez, o vetor das incógnitas tem o aspecto:

$$
X=\left\{\begin{array}{l}
c_{1} \\
c_{2} \\
d_{\boldsymbol{t}}
\end{array}\right\}=\left\{\begin{array}{l}
c_{1} \\
c_{2} \\
d_{1} \\
d_{2} \\
d_{3} \\
d_{4} \\
d_{5} \\
d_{6}
\end{array}\right\}
$$

onde $\boldsymbol{d}_{\boldsymbol{k}}{ }^{T}=\left\{u_{x k} \quad u_{y_{k}}\right\}$, para $k=1, \cdots, 6$.

Ressalta-se que para o sistema da Eq. (C.1) admitir solução é necessário a verificação do teste do mosaico. Além disso, tendo-se em vista que os nós 1 e 4 estão impedidos, deve-se zerar as linhas e colunas do sistema global correspondentes a estas posições.

- Verificação ao equilíbrio de forças nodais equivalentes

A imposição da continuidade das forças nodais equivalentes pode ser constatada, considerando-se a terceira linha do sistema ditado pela Eq. (C.1), assim expressa: 


$$
-G_{1}^{T} c_{1}-G_{2}^{T} c_{2}=-q_{t}
$$

Considerando-se a Tabela C1 e aplicando-se as condições de contorno aos nós 1 e 4, a Eq. (C.12) adquire a forma:

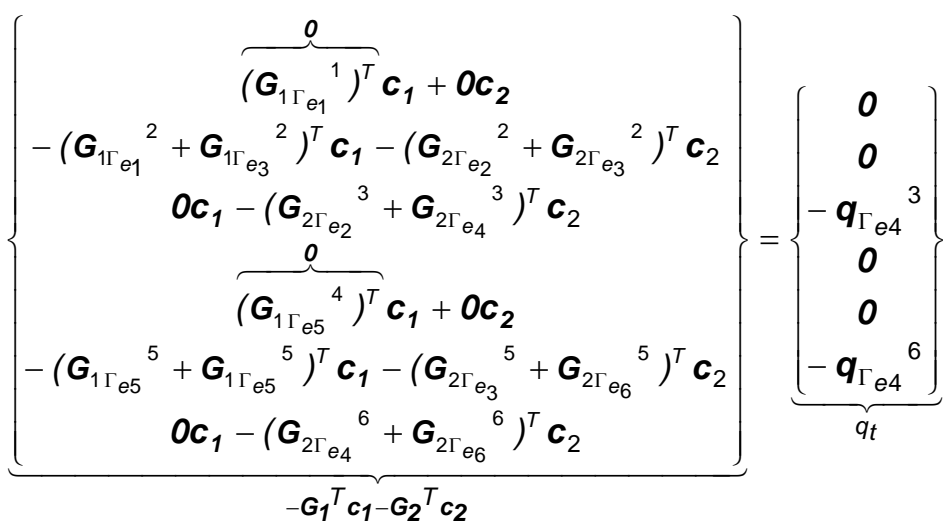

Considerando-se as matrizes envolvidas desde a Eq.(C.2) até a Eq.(C.8) e as aproximações $\boldsymbol{t}_{\boldsymbol{1}}=\boldsymbol{T}_{1} \boldsymbol{c}_{\boldsymbol{1}}$ e $\boldsymbol{t}_{\boldsymbol{2}}=\boldsymbol{T}_{2} \boldsymbol{c}_{2}$ relativas, respectivamente, a aproximação dos campos de tensões no contorno dos elementos, a Eq.(C.13) pode ser reescrita como:

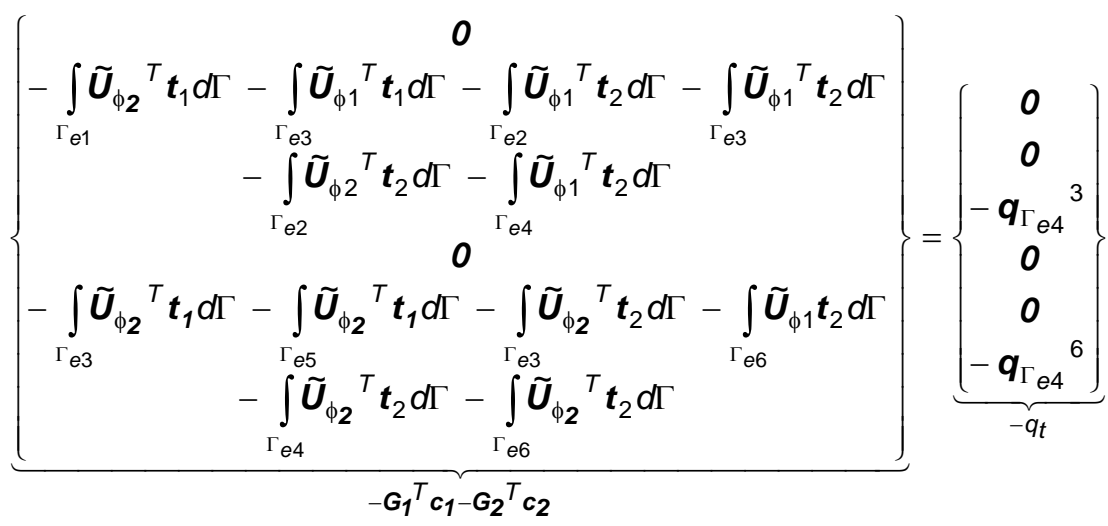

Verifica-se que a Eq. (C.14) representa em essência uma condição de equilíbrio nodal. Ela estabelece que, em cada nó não restrito da discretização, as forças nodais equivalentes provenientes das tensões devem ser iguais às provenientes do carregamento. Por conseguinte, a formulação híbrida-Trefftz promove uma boa continuidade na aproximação de tensões em toda estrutura. 


\section{Apêndice D - Exemplo de montagem do sistema resolutivo com enriquecimento}

Com o propósito de exemplificar a montagem do sistema global e a verificação ao equilíbrio de tensões na interface dos elementos, quando da formulação híbrida-Trefftz com enriquecimento, considere-se a mesma chapa analisada no apêndice C. Entretanto, admita-se que os trechos $\Gamma_{\text {e3 }}$ e $\Gamma_{\text {e4 }}$ sejam enriquecidos, conforme indicado na Figura D.1.

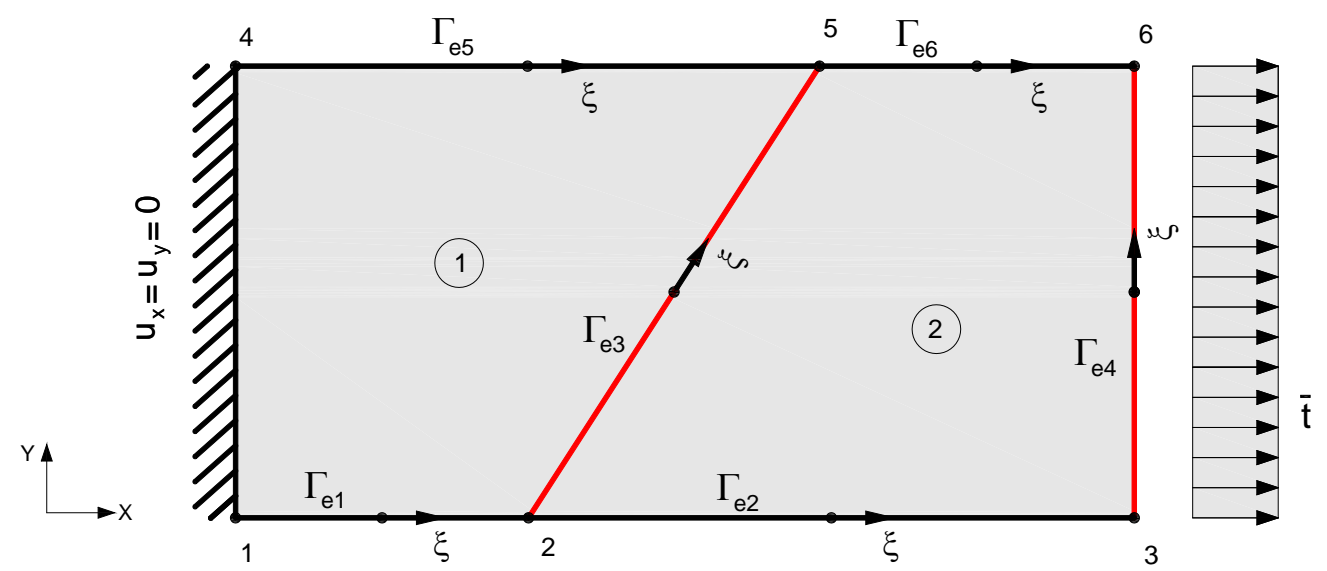

Figura D.1. Chapa discretizada em dois elementos com dois trechos enriquecidos.

Assim sendo, o sistema global fica com a forma:

$$
\boldsymbol{P}^{\prime} \boldsymbol{X}^{\prime}=\boldsymbol{Q}^{\prime}
$$

sendo $P^{\prime}=\left(\begin{array}{cccc}F_{1} & 0 & -G_{1}-G_{1} \\ 0 & F_{2} & -G_{2} & -G_{2} \\ -G_{1}{ }^{T} & -G_{2}{ }^{T} & 0 & 0 \\ -G_{1}{ }^{T} & -G_{2}{ }^{T} & 0 & 0\end{array}\right), X^{\prime}=\left\{\begin{array}{c}c_{1} \\ c_{2} \\ d_{t} \\ d_{t}\end{array}\right\}$ e $Q^{\prime}=\left\{\begin{array}{c}e_{1} \\ e_{2} \\ -q_{t} \\ -q_{t}\end{array}\right\}$.

- Montagem

A montagem da matriz $\boldsymbol{P}^{\prime}$ pode ser realizada, acrescentando-se ao processo de montagem da matriz $\boldsymbol{P}$ (vide Apêndice C), as matrizes $\boldsymbol{G}_{1}$ e $\boldsymbol{G}_{2}$, decorrentes do enriquecimento. Elas são alocadas pelo compartilhamento dos graus de liberdade em deslocamentos do sistema, considerando-se cada trecho enriquecido da rede. 
Por exemplo, a parcela de $G_{1}$ referente ao trecho $\Gamma_{\mathrm{e} 3}$, pode ser escrita por:

$$
\boldsymbol{G}_{1 \Gamma_{e 3}}=\int_{\Gamma_{e 3}} \boldsymbol{T}_{1}^{T} \tilde{\boldsymbol{U}}_{\Gamma_{e 3}} d \Gamma
$$

Analogamente, para as outras situações, escreve-se:

Parcela de $G_{2}$ em $\Gamma_{\mathrm{e} 3}$ :

$$
\boldsymbol{G}_{2 \Gamma_{e 3}}=\int_{\Gamma_{e 3}} \boldsymbol{T}_{2}^{T} \tilde{\boldsymbol{U}}_{\Gamma_{e 3}} d \Gamma
$$

Parcela de $G_{2}$ em $\Gamma_{\mathrm{e} 4}$ :

$$
\boldsymbol{G}_{2 \Gamma_{e 4}}=\int_{\Gamma_{e 4}} \boldsymbol{T}_{2}^{T} \tilde{\boldsymbol{U}}_{\Gamma_{e 4}} d \Gamma
$$

Na montagem do vetor $\boldsymbol{Q}^{\prime}$ o processo é análogo. Acrescenta-se ao vetor $\boldsymbol{Q}$ a parcela $\boldsymbol{q}_{t}$, proveniente do enriquecimento.

A única parcela não nula de $\boldsymbol{q}_{\boldsymbol{t}}$ resulta do trecho carregado $\Gamma_{\mathrm{e} 4}$, sendo dada por:

$$
\boldsymbol{q}_{\Gamma_{e 4}}=\int_{\Gamma_{e 4}} \tilde{\boldsymbol{U}}_{\Gamma_{e 4}}{ }^{\top} \overline{\boldsymbol{t}} d \Gamma
$$

Por sua vez, o vetor das incógnitas $\boldsymbol{X}^{\prime}$ pode ser obtido acrescentando-se os vetores $\boldsymbol{d}_{1}$ e $\boldsymbol{d}_{2}$, relativos, respectivamente, aos trechos enriquecidos $\Gamma_{\mathrm{e} 3}$ e $\Gamma_{\mathrm{e} 4}$, ao vetor $\boldsymbol{X}$.

Admitindo-se que em $\Gamma_{\mathrm{e} 3}$ e $\Gamma_{\mathrm{e} 4}$ sejam acrescentados, respectivamente $\Delta \mathrm{n}_{\mathrm{d} 1}$ e $\Delta \mathrm{n}_{\mathrm{d} 2}$ graus de liberdade, têm-se:

$$
\begin{aligned}
& \boldsymbol{d}_{1}^{T}=\left\{\begin{array}{lllll}
\left(\Delta d_{11 x}\right. & \Delta d_{11 y}
\end{array}\right) \cdots\left(\begin{array}{lll}
\Delta d_{1 \Delta n_{d 1} x} & \Delta d_{1_{\Delta n_{d 1} y} y}
\end{array}\right\} \\
& \left.\boldsymbol{d}_{2}{ }^{T}=\left\{\begin{array}{lllll}
\left(\Delta d_{21 x}\right. & \left.\Delta d_{21 y}\right) & \cdots & \left(\Delta d_{2 \Delta n_{d 2} x}\right. & \Delta d_{2 \Delta n_{d 2} y}
\end{array}\right)\right\}
\end{aligned}
$$

A partir das considerações anteriores o sistema global admite o aspecto indicado na Tabela D.1. Nela, por simplicidade, as matrizes $G_{1}$ e $G_{2}$ estão representadas de forma genérica. Evidentemente, elas devem ser alocadas conforme exposto no apêndice C.

Ressalta-se que a solução do sistema ditado pela Eq. D.1 é possível, desde que sejam impostas as condições de contorno aos nós 1 e 4 e seja verificada, em cada elemento e em todos os possíveis "mosaicos" de elementos, a condição de estabilidade, evidentemente considerando-se o enriquecimento. 
Tabela D.1 - Montagem do sistema resolutivo com enriquecimento

\begin{tabular}{|c|c|c|c|c|c|c|c|c|}
\hline \multicolumn{5}{|c|}{ Colunas de referência para montagem } & & & & \\
\hline \multicolumn{2}{|c|}{$\begin{array}{c}\begin{array}{c}\text { Graus de liberdade em } \\
\text { tensões }\end{array} \\
\end{array}$} & \multicolumn{3}{|c|}{$\begin{array}{c}\begin{array}{c}\text { Graus de liberdade em } \\
\text { deslocamentos }\end{array} \\
\end{array}$} & & & & \\
\hline \multirow[t]{2}{*}{$c_{1}$} & \multirow[t]{2}{*}{$c_{2}$} & \multirow[t]{2}{*}{$\mathbf{d}_{\mathrm{t}}$} & $\begin{array}{c}\text { Trecho } \\
\Gamma_{\mathrm{e} 3} \\
\end{array}$ & $\begin{array}{c}\text { Trecho } \\
\Gamma_{\mathrm{e} 4}\end{array}$ & & & & \\
\hline & & & $\mathbf{d}_{1}$ & $d_{2}$ & & & & \\
\hline \multicolumn{9}{|c|}{ Montagem do sistema $\mathbf{P}^{\prime} \mathbf{X}^{\prime}=\mathbf{Q}^{\prime}$} \\
\hline \multicolumn{5}{|c|}{$\mathbf{P}^{\prime}$} & & $\mathbf{X}^{\prime}$ & & $\mathbf{Q}^{\prime}$ \\
\hline$F_{1}$ & 0 & $-\mathbf{G}_{\mathbf{1}}$ & $-\mathbf{G}_{{ }_{{ }^{1}} 3}$ & 0 & & $c_{1}$ & \multirow{5}{*}{$=$} & 0 \\
\hline 0 & $\mathbf{F}_{2}$ & $-G_{2}$ & $-\mathbf{G}_{2 \Gamma_{\mathrm{e} 3}}$ & $-\mathbf{G}_{2 \Gamma_{\mathrm{e} 4}}$ & & $c_{2}$ & & 0 \\
\hline$-\mathbf{G}_{1}^{\top}$ & $-\mathbf{G}_{2}^{\top}$ & \multirow{3}{*}{\multicolumn{3}{|c|}{0}} & $x$ & $d_{t}$ & & $-q_{t}$ \\
\hline$-\mathbf{G}_{1 \Gamma_{\mathrm{e} 3}}{ }^{\top}$ & $-\mathbf{G}_{2 \Gamma_{\mathrm{e} 3}}{ }^{\mathrm{T}}$ & & & & & $\mathbf{d}_{1}$ & & 0 \\
\hline 0 & $-\mathbf{G}_{2 \Gamma_{\mathrm{e} 4}}{ }^{\mathrm{T}}$ & & & & & $d_{2}$ & & $\mathbf{q}_{\Gamma_{\mathrm{e} 4}}$ \\
\hline
\end{tabular}

\begin{tabular}{|l|l|}
\hline & Matrizes: $-\mathbf{G}_{\mathbf{1}} \mathrm{e}-\mathbf{G}_{\mathbf{1}}{ }^{\top}$ \\
\hline & Matrizes: $-\mathbf{G}_{\mathbf{2}} \mathrm{e}-\mathbf{G}_{\mathbf{2}}{ }^{\top}$ \\
\hline & Vetor: - $\mathbf{q}_{\mathbf{t}}$ \\
\hline
\end{tabular}

- Verificação ao equilíbrio de tensões nos trechos enriquecidos:

Verifica-se que o equilíbrio de tensões também está presente na formulação híbridaTrefftz com enriquecimento. Agora, ele é estabelecido em forma fraca nos trechos enriquecidos.

De fato, considerando-se a quarta linha do sistema ditado pela Eq. (D.1), escreve-se:

$$
\text { - } G_{1}{ }^{T} c_{1}-G_{2}{ }^{T} c_{2}=-q_{t}
$$

De acordo com a tabela D.1, a Eq. (D.8) adquire o aspecto:

$$
\underbrace{\left\{\begin{array}{c}
-\boldsymbol{G}_{1 \Gamma_{e 3}}{ }^{T} \boldsymbol{c}_{\boldsymbol{1}}-\boldsymbol{G}_{2 \Gamma_{e 3}}{ }^{T} \boldsymbol{c}_{\boldsymbol{2}} \\
\boldsymbol{0} \boldsymbol{c}_{\boldsymbol{1}}-\boldsymbol{G}_{2 \Gamma_{\Gamma}{ }^{T} \boldsymbol{c}_{2}}
\end{array}\right\}}_{-\boldsymbol{G}_{1}{ }^{T} \boldsymbol{c}_{\mathbf{1}}-\boldsymbol{G}_{\mathbf{2}}{ }^{T} \boldsymbol{c}_{\mathbf{2}}}=\underbrace{\left\{\begin{array}{c}
\boldsymbol{0} \\
-\boldsymbol{q}_{\Gamma_{e 4}}
\end{array}\right\}}_{-q_{t}} .
$$

que a partir do conjunto de equações (da Eq. (D.2) à Eq. (D.5)) e das aproximações $\boldsymbol{t}_{\boldsymbol{1}}=\boldsymbol{T}_{1} \boldsymbol{c}_{\boldsymbol{1}} \mathrm{e}$ $\boldsymbol{t}_{\mathbf{2}}=\boldsymbol{T}_{2} \boldsymbol{c}_{2}$ relativas, respectivamente, a aproximação dos campos de tensões no contorno dos elementos, pode ser reescrita como: 


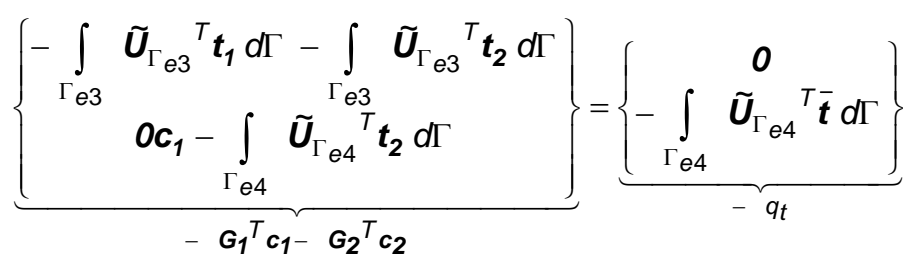

A Eq. (D.10) leva a duas relações de interesse, quais sejam:

$$
\begin{aligned}
& \int_{\Gamma_{e 3}} \tilde{\boldsymbol{U}}_{\Gamma_{e 3}}{ }^{T}\left(\boldsymbol{t}_{\mathbf{1}}+\boldsymbol{t}_{\mathbf{2}}\right) d \Gamma=\mathbf{0} \\
& \int_{\Gamma_{e 4}} \tilde{\boldsymbol{U}}_{\Gamma_{e 4}}{ }^{T}\left(\boldsymbol{t}_{\mathbf{2}}-\overline{\boldsymbol{t}}\right) d \Gamma=\mathbf{0}
\end{aligned}
$$

A primeira estabelece, de forma fraca, a continuidade de tensões na interface dos elementos. Observa-se que os pesos são as funções utilizadas na aproximação do campo de deslocamentos no contorno. De maneira análoga, a segunda promove o equilíbrio entre as tensões e as forças de superfície na face carregada. Daí percebe-se a importância do enriquecimento na continuidade da aproximação de tensões. 